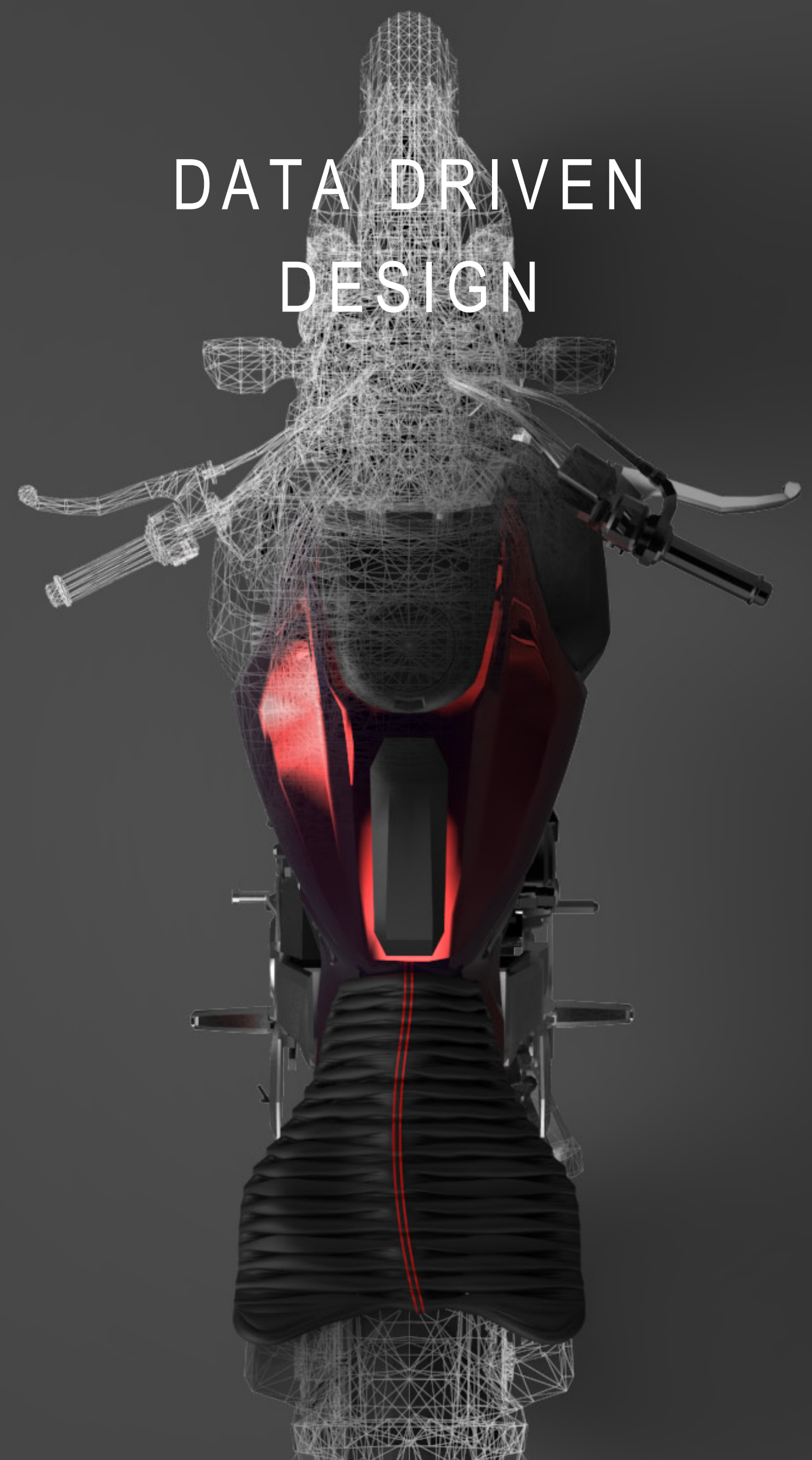

An investigation into the fit between the individuality of people and the uniformity of mass manufactured

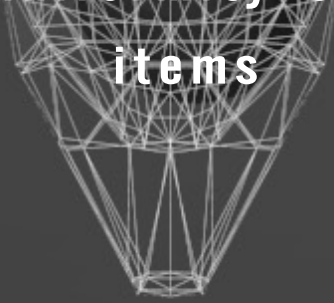




\section{DATA DRIVEN DESIGN}

An investigation into the fit between the individuality of people and the uniformity of mass manufactured items

Alex Svend Christensen

A 90 point thesis submitted in fulfilment of the requirements for the Masters of Design Innovation, School of Design, Victoria University of Wellington. 


\section{ACKNOWLEDGEMENT}

There are many people I would like to acknowledge who have given me support and reassurance over the course of my thesis.

Firstly I would like to thank my Mum, Dad, Brother, and the rest of my family. Their endless backing and encouragement helped keep me optimistic when things weren't going right.

To my friends, peers and classmates. We all know the struggle of the thesis. You have easy weeks, hard weeks, and weeks where you want to give up. I would like to thank every one of you who helped me keep my head high along this journey. Specifically James, Zara, and Feng, I want to thank you for your endless banter helping me to maintain the balance between work and fun.

I would like to thank Phil for continuously assisting me with the use of the Big Rep 3D printer and being patient with the endless amount of failures.

Lastly to my supervisors Bernard, Ross, and the rest of the Victoria University staff who have assisted me through this research. Thank you for giving me advice and allowing me to pick your brains. I have had many looks of confusion while explaining my ideas to some of you but thank you for sticking by me and giving me feedback and inspiration. 
This thesis sets out to apply 3D scanning and human scale $3 \mathrm{D}$ printing to the design of personalised seating. 


\section{ABSTRACT}

Due to the economic advantage of mass manufacturing technology humans have designed a world of products built for the average body size and shape. This conformity of diverse body shapes to fixed 3 dimensional forms raises the question for this research; how can 3D scanning and additive manufacturing (AM) create a personal fit between an individual's body and a product?

This question challenges a tool driven standardised approach to manufacture by exploring the interface between a person and a mass produced product, in this case a motorcycle rider and a motorcycle. By taking advantage of digital data and the tool-less build process of 3D printing, every object produced can be different, tailoring it to the customer's individual aesthetic or physical fit.

This investigation into the space between the motorcycle and the human has produced a custom 3D printed seat designed for and inspired by the unique physicality of the individual rider. The following methods are employed. 3D scanning is used to obtain the geometry of the human form and motorcycle, 3D modelling and 3D printing to generate and evaluate ideas and concepts, and a pressure measurement system to evaluate the riders comfort and fit.

This new relationship between body and object, rarely seen in mass produced products, questions the way we design and make products with consideration towards digital personalisation and manufacturing efficiency. 


\section{CONTENTS}

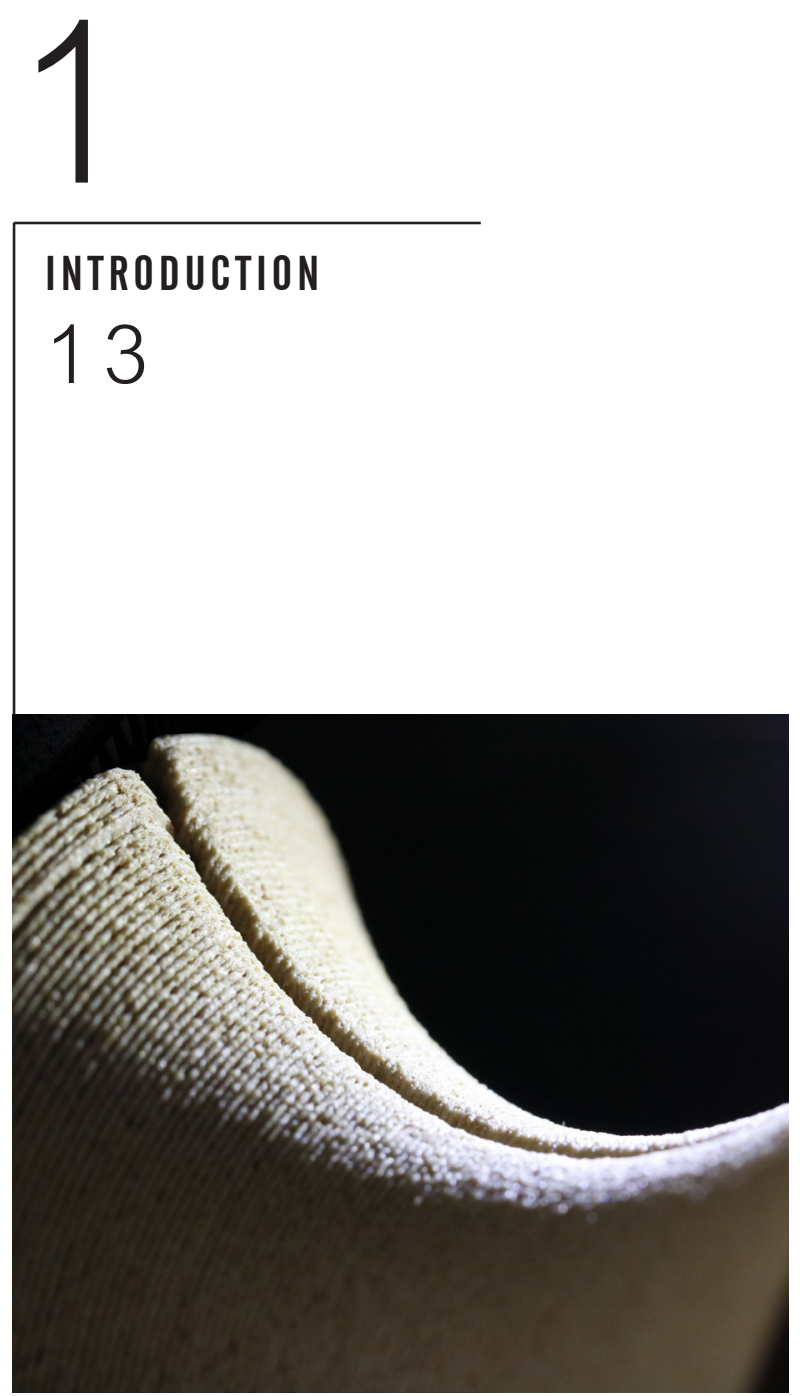

\section{LITERATURE REVIEW}

19

22| Case Study

24| 3D Printing

30| 3D Scanning

31| Anthropometric Fit

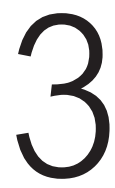

METHODOLOGY

35

36| Research Through 38| Research Methods 44| Materials

45| Equipment
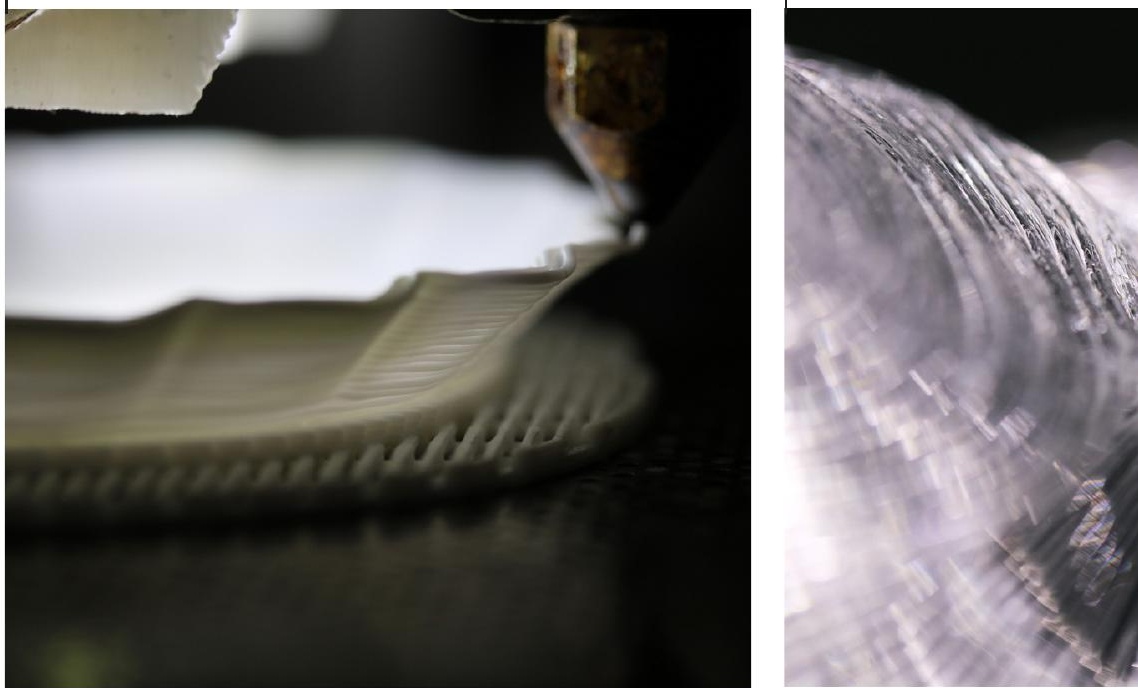


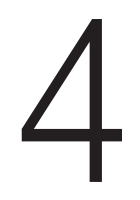

\section{DESIGN PROCESS}

\section{7}

50| Initial Experimentation

72| Application

78| Support Testing

80| Full Scale Prototyping

110| Post Processing

114| Participant Evaluation

118| Aesthetic Opportunities

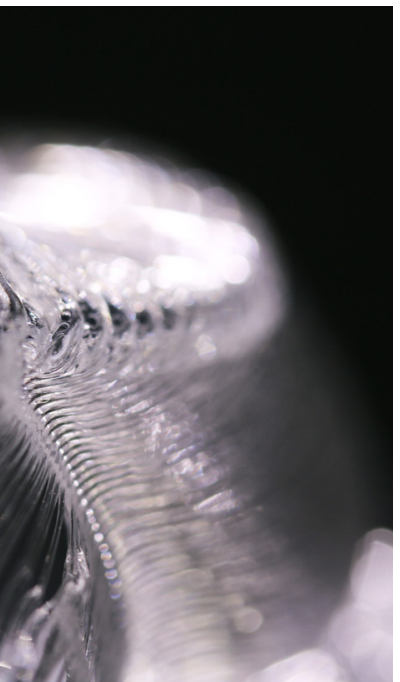

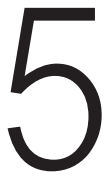

CRITICAL REFLECTION + CONCLUSION 120

123| Methods

125| Aesthetic Decisions

126| Evaluation

127| Real World Application

127| Limitations

130| Figures

132| Bibliography

134| Appendix
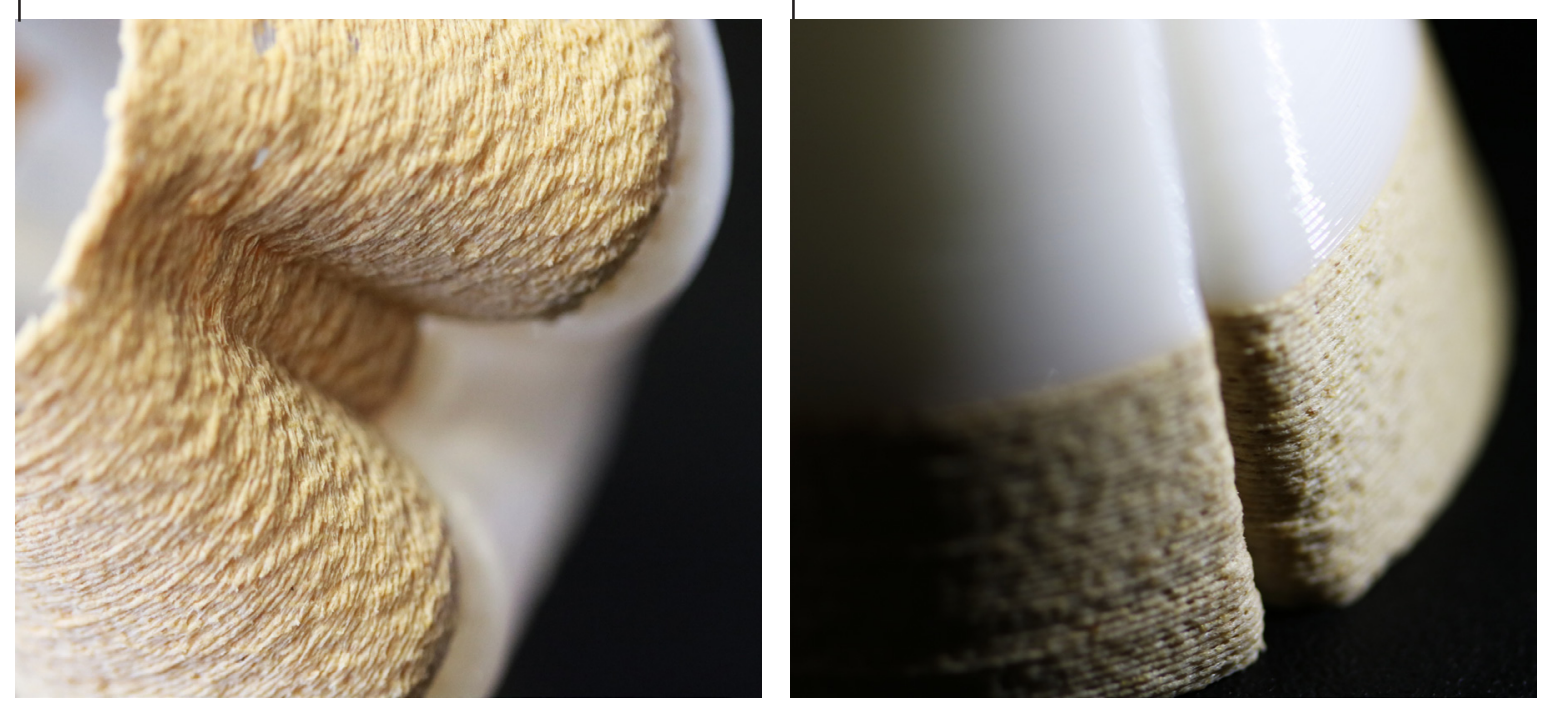


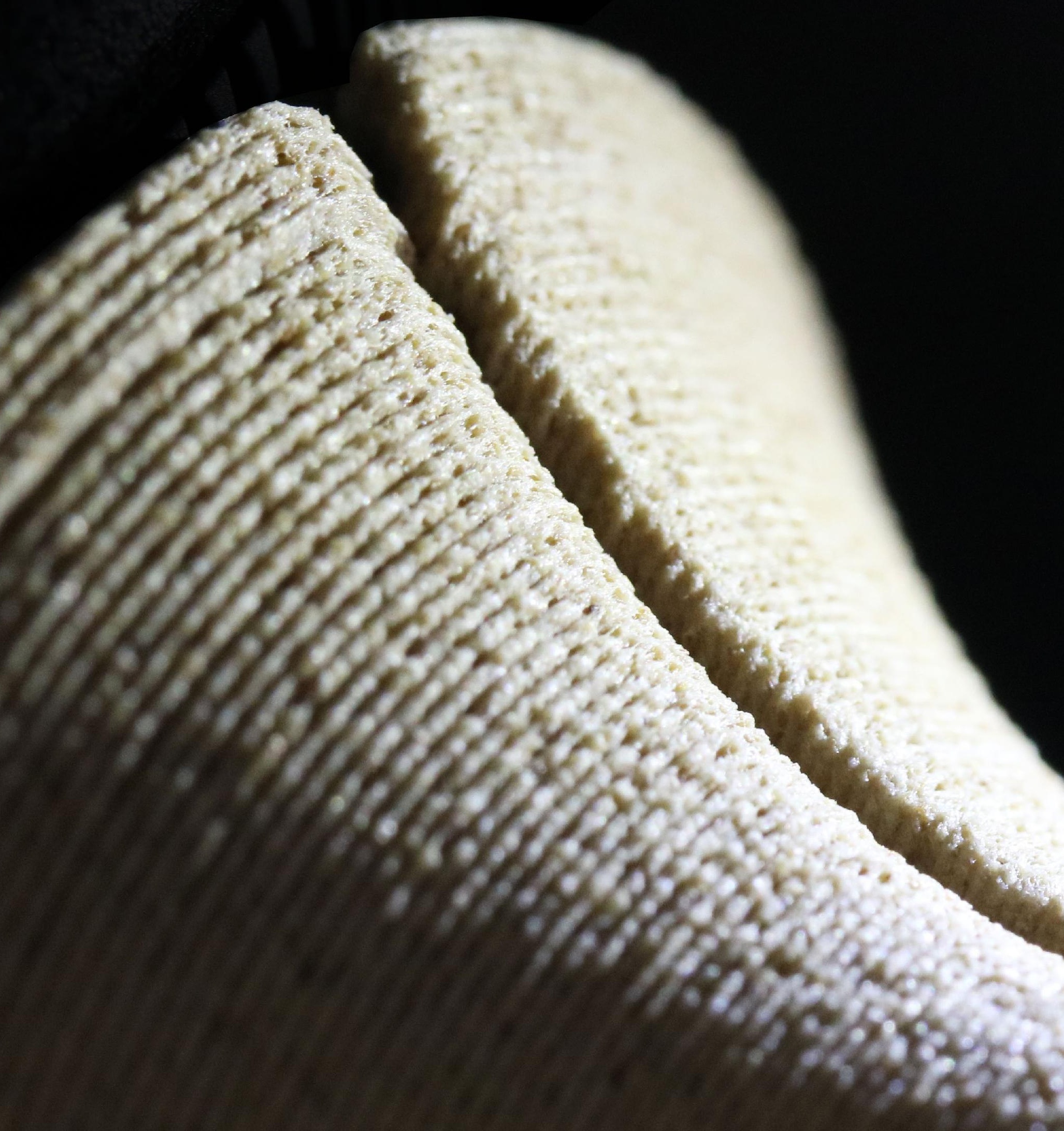

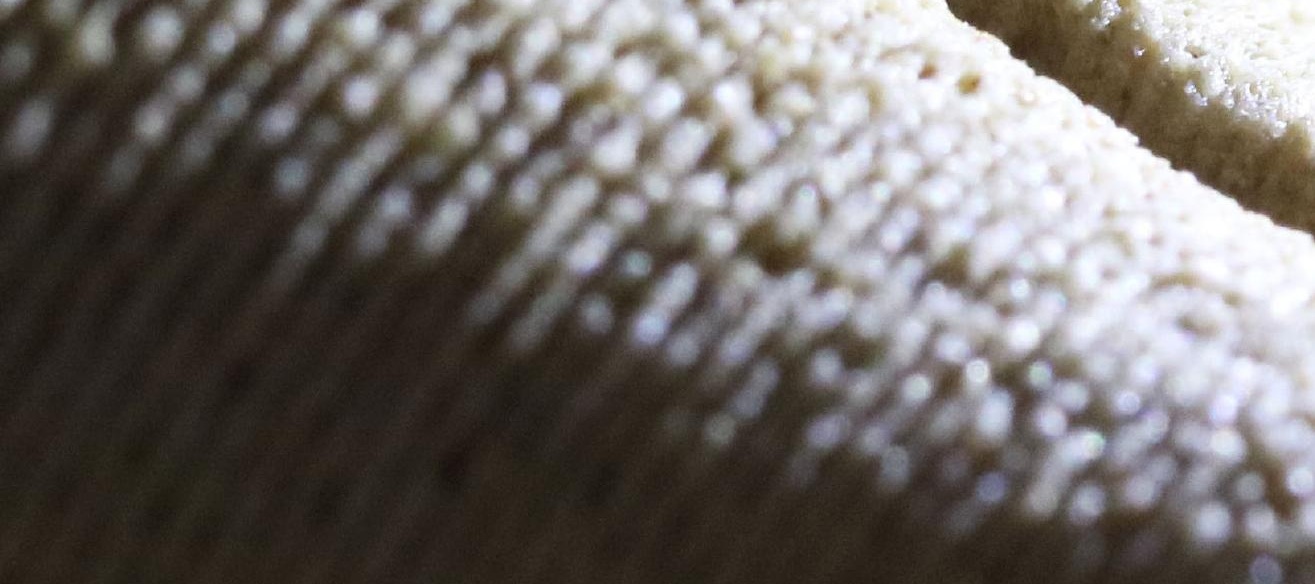




$$
1
$$




\section{INTRODUCTION}

Due to the economic advantage of mass manufacturing technology humans have designed a world of products built for the average body size and shape. This conformity of diverse body shapes to fixed 3 dimensional forms raises the question for this research; how can 3D scanning and additive manufacturing (AM) create a personal fit between an individual's body and a product?

3D printing, a relatively new manufacturing process, takes advantage of low investment costs when compared to mass production technology. Additionally, 3D printing allows for customisation and personalisation within the objects created whilst maintaining a production cost efficiency (Figure 1) (Barnatt, 2014). Every object produced by a 3D printer can be different, tailoring it to the customer's individual aesthetic and form preference, similar to bespoke handcrafted objects. Unlike handcrafted products, additive manufacturing takes advantage of low production costs and time when associated with mass manufacturing allowing objects traditionally used as prototypes to be sold directly to the customer.

Currently, most of our everyday products are generically designed to be mass manufactured using technologies such as injection moulding. Large product runs provided by this technology allow cheap and fast production however do not accommodate individuality. This type of traditional manufacturing does not function well with the small product runs associated with customised objects. Large format multi-property $3 \mathrm{D}$ printing is very new and not only allows but encourages the production of personalised goods. Universally, the development of industrial scale manufacturing technologies, up until now, has been very limiting towards giving people customised product options. The potential exists for 3D printers to alleviate this domination.

Additive manufacturing is defined for this research as fused deposition modelling (FDM) 3D printing, a process where an object is built by molten plastic being deposited layer upon layer. While there is an abundance of 3D printed objects manufactured using low cost FDM 3D printing, few academic studies have examined the potential of manufacturing products, with an anthropometric fit (Wohlers, 2014). This project acknowledges this knowledge gap by investigating the material and aesthetic capabilities of FDM printing and its ability to mass produce customised products.

This research explores the fit between body and object through the application of a motorbike seat which is personally fitted to a single user. Focusing on an application which is commonly designed to a generic body size percentile allows for an exploration into designing custom fit motorcycle seating using 3D scanning and 3D printing (Robertson \& Minter, 1996). The personal fit between body and object, created through the use of these two technologies, is something rarely seen in mass produced objects. It is an advantageous opportunity, in a world build around generic objects, to be able to produce a unique motorcycle seat designed using a rider's individual form, at an economical cost when compared to traditional, handmade, customised seating. 

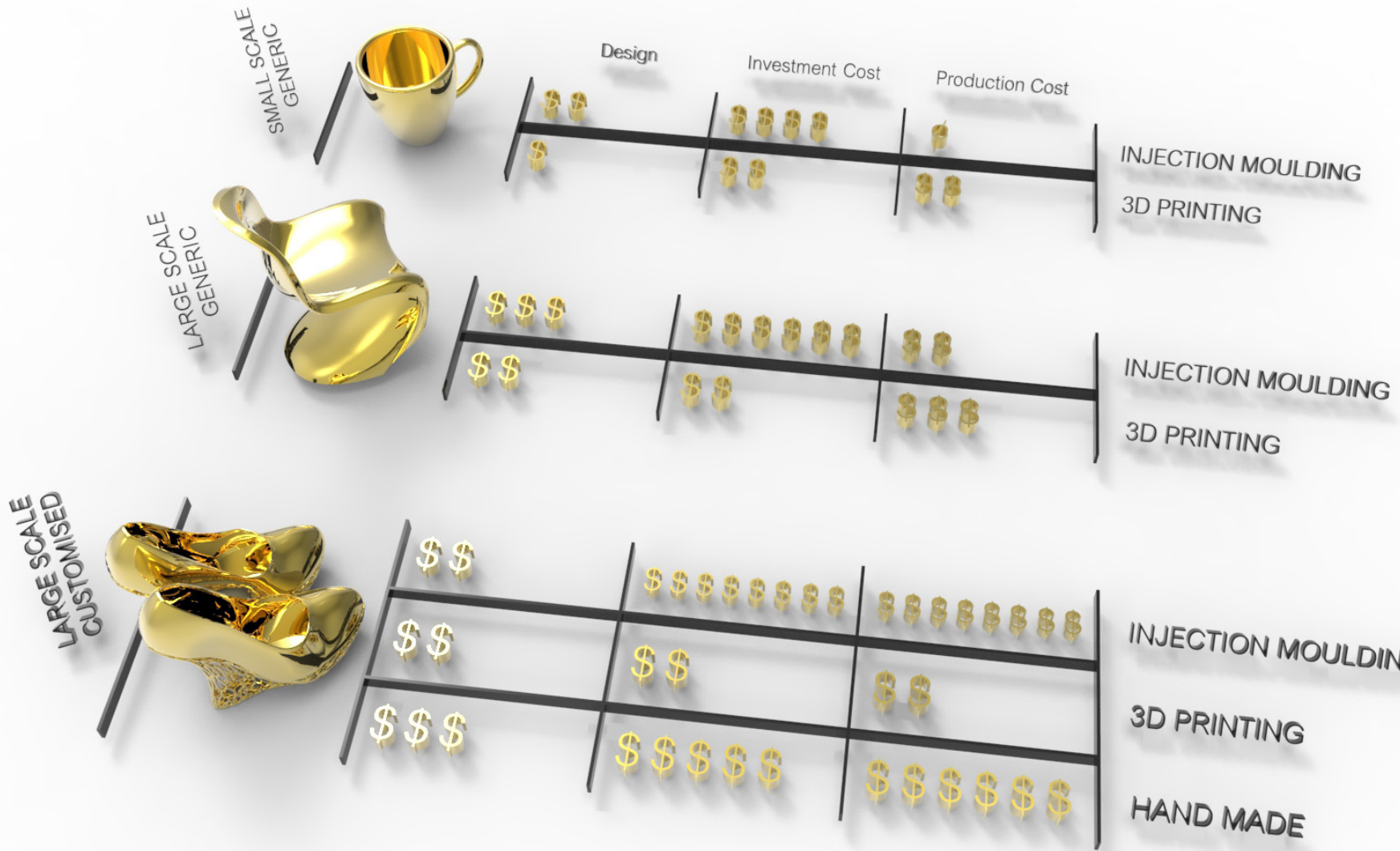

INJECTION MOULDING

3D PRINTING

HAND MADE

Figure 1 - Diagram comparing the efficiency of injection moulding and 3D printing when creating generic, and customised objects at different scales. 
This research uses a research through design (RTD) approach, a methodology which employs methods and processes from design practice as legitimate methods of inquiry (Zimmerman, Forlizzi, \& Evenson, 2007). The act of making forces the designer to focus on developing the research following an iterative process to create calculated decisions. RTD is applied within this research to explore ideas, determine applications, and resolve problems. It allows the research to create visual and tactile start points to develop ideas and evaluate both personally and with others. It also allows a visual history of ideas to be documented and reflected on, providing improvement to the design whilst reducing assumption within design decisions.

This research uses sketching, CAD modelling, low and high fidelity prototyping, rendering and photographic documentation as part of the design process. A text commentary communicates how and why discussions have been made, reflecting on each part of the process. Figure 2 illustrates the design process within this research.

\section{Research Methods}

\section{PROTOTYPING/IDEATION}

- Low fidelity prototypes are quick iterative tools used to test early ideas generating research and concepts (Gaver, 2012). These may include sketches, renders, and models used to visualise and develop a design (Zimmerman, Forlizzi, \& Evenson, 2007).

- High fidelity prototypes are "more refined often representing the appearance of the final product in look and feel and sometimes even basic functionality" (Gaver, 2012, p.937). They give the designers and reviewing participants an accurate model to test and evaluate.

\section{D SCANNING}

3D scanning captures complex three dimensional form as digital data which can be manipulated through CAD software. This technology can efficiently scan the human body in the weight bearing situation of riding a motorcycle. 3D scanning allows for an exploration into personal ergonomic form investigating the shape of the body in relation to the physicality of a motorcycle.

\section{D PRINTING}

$3 \mathrm{D}$ printing is a tool used to visualise, develop, and test an idea. It can be used to manufacture small low fidelity models used to develop basic ideas as well as highly detailed prototypes used for testing. FDM 3D printing is employed within this research as it is low-cost, and has the capability of dual-material 3D printing. 


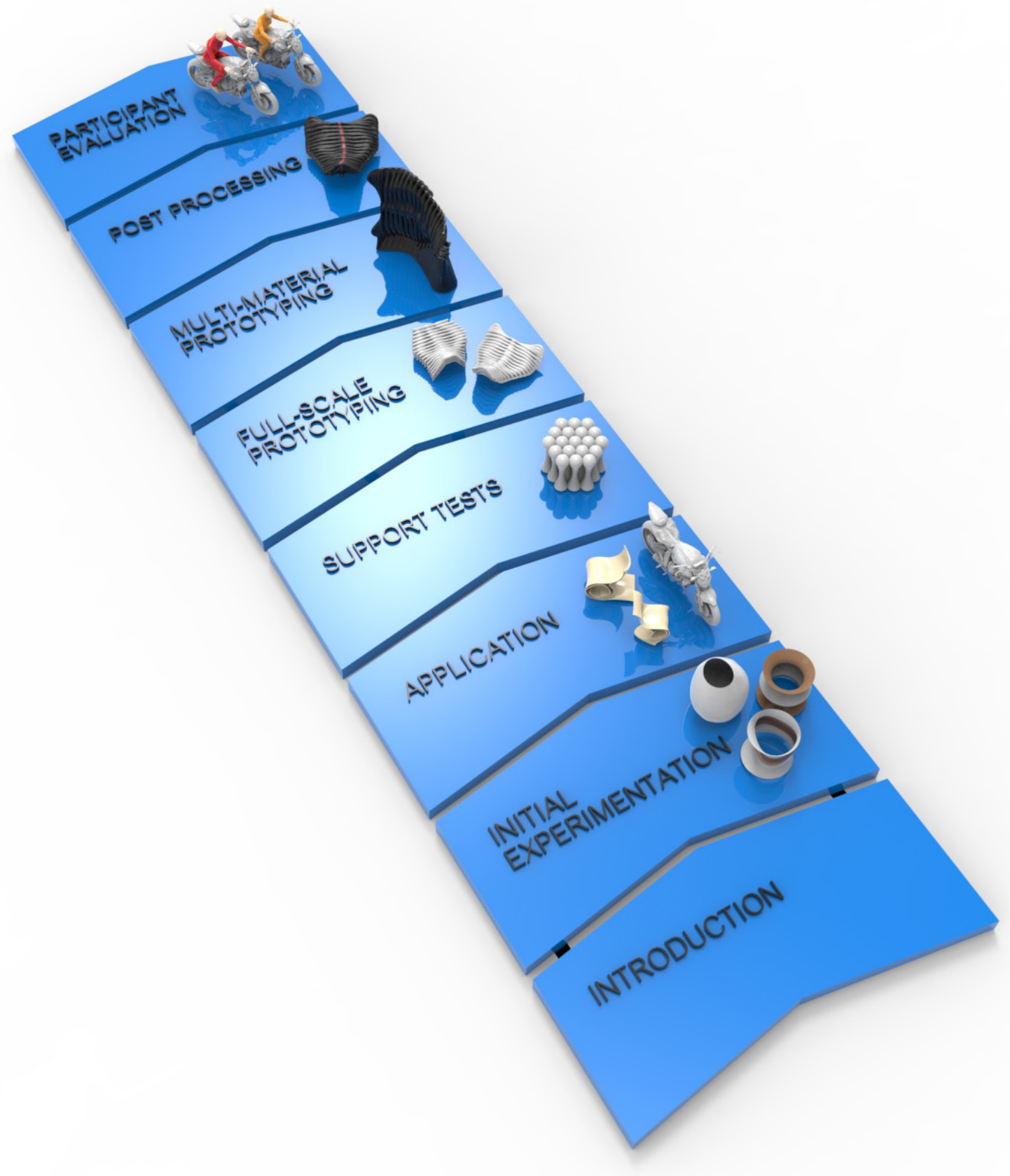

Figure 2 - Plan of how the research section of this thesis has been approached 


\section{Manufacturing technologies}

- Within this research mass manufacturing or mass production is a term that describes the traditional manufacturing methods that are used to make universally shaped products. Injection moulding technology is one example, where large quantities of generic objects are produced over a short period of time on a 'mass' scale (Thompson, 2007).

- Mass Customisation, for this research, is a term that explains how 3D printing can be used to build customised objects efficiently, having low investments costs and taking advantage of the high product output associated with mass production. 


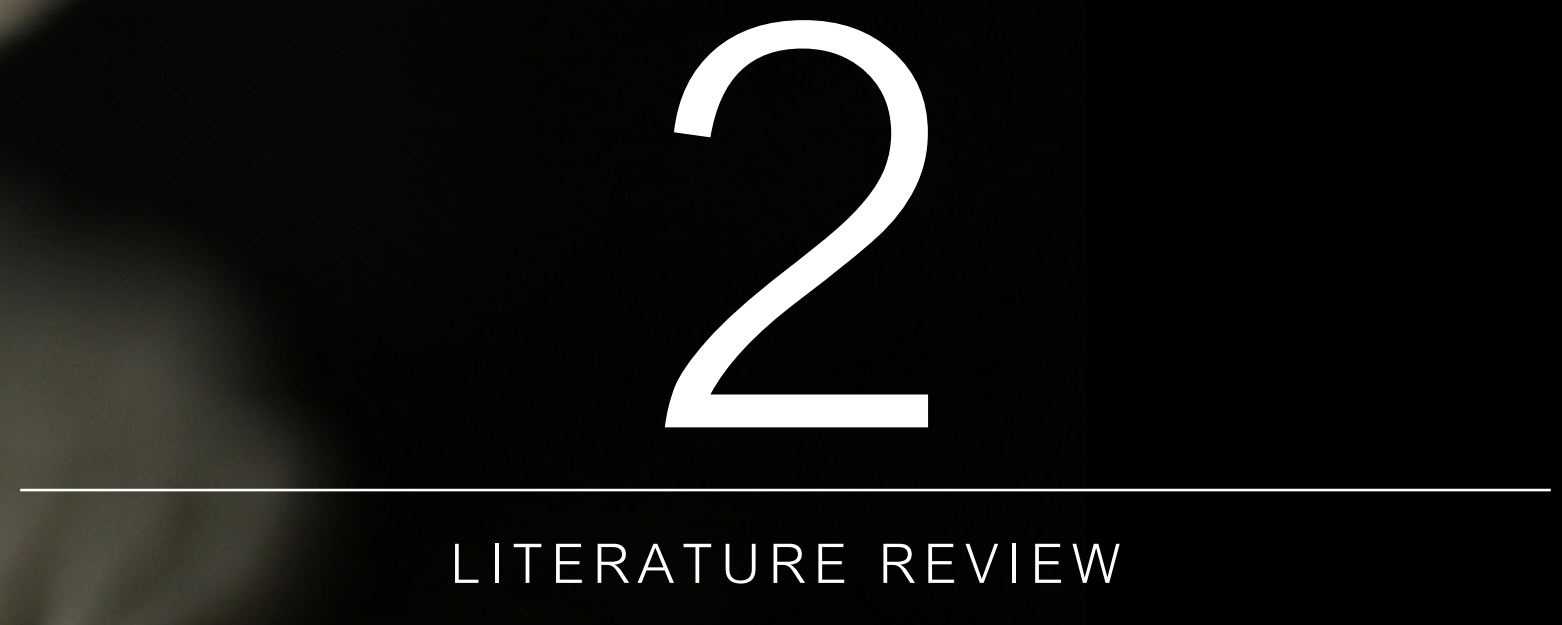




\section{LITERATURE REVIEW}

Distilling the information from current examples of large scale FDM 3D printing, as well as projects involving 3D scanning technology, captures the essence of the investigated design field. This research focuses on combining these technologies, making it important to look at published sources as well as a variety of real-world, physical examples. This allows connections to be made between the two technologies as well as building an archive of reputable precedents.

The 'Panton Chair' is a cantilevered seat designed by Verner Panton. This chair demonstrates a "willingness to experiment with new materials and his determination to be the first designer to exclusively create cantilevered seating using a single material while needing only one form of tool to do so" (Albus et al., 2013, p. 409). As a method of research through making, this precedent was designed and constructed at a time when injection moulding technology and the development of plastics were very new, similar to the current paradigm around 3D printing technologies, holding comparable aspirations.

It is important to review the literature around how FDM 3D printing and 3D scanning has been used in current projects and research. This gives insight into the void this research fills as well as the limitations of materials, and how this new technology can enforce an opportunity for change in design. 
This research draws on material and manufacturing explorations Verner Panton under took when designing the Panton chair. Preferring the challenge of new technologies to the handcraft traditions of the Danish designers of the mid-20th century, Panton took a different approach with interest into "experimenting with materials, particularly synthetics" (Watson, 2002, p. 19). The chair Panton is most well-known for is the 'Panton Chair', "a stackable cantilevered dining chair whose base, seat, and back were to be constructed from a single piece of material" (Figure 2) (Hausfeld \& Hvidberg-Hansen, 2013, p. 76). The young Danish designer began dreaming up this chair in the early 1950s. This type of seating design had not been built before.

Panton's material explorations and manufacturing development has a fundamental relationship to the methodology this research follows. The theory behind the development of the Panton chair may aid the advancement of 3D printing technologies and materials, influencing a change to the design and manufacturing of seating.

Material experimentation was Panton's passion. Continuously and deliberately disregarding established methods and tradition, Panton preferred a unique approach to his work which was different from that of his colleagues. Panton started by creating non-structural extruded polystyrene seating prototypes and later moved to a stronger, durable polyurethane hard foam plastic. Although this material had a different moulding process to some other plastics, it "made it possible to produce large quantities of the product but did not eliminate manual finishing" (Hausfeld \& Hvidberg-Hansen, 2013, p. 85) consequently pushing up the price. Material developments related directly to the manufacturing technology of the time.

In the 1950s and 1960s injection moulding technologies were very new with limited functions (Pina, 2000). Previously structural furniture was built using traditional wooden construction. This made it much more difficult for Panton, as he was not only trying to design an incredibly complex seating shape but also build and advance the technology within the manufacturing process as well. Gerrit Rietveld, a Dutch furniture designer and architect, had tried to create a single-form chair and failed due to the lack of technology at the time. Panton's drive to succeed, despite his failures, eventually paid off when his chair was "credited with the first single-form injection moulded plastic chair" (Pina, 2000, p. 55). The injection moulding technology at the time was only able to produce a uniform width which meant that they were unable to thicken some areas of the chair to provide extra strength. This forced "parallel ribs to be added to the underside of the chair" (Hausfeld \& Hvidberg-Hansen, 2013, p. 85).

Over the two decades of design development, plastics and manufacturing technologies were developed and refined easing the moulding and manufacturing of the Panton chair (Hausfeld \& Hvidberg-Hansen, 2013). Reducing the material and manufacturing limitations improved efficiency in producing a chair which was economically viable and ultimately successful. 3D printing is developing in similar way to the injection moulding technology Panton helped establish. Through recent advances in the quality and efficiency of 3D printing technology, models can now be sold directly from the printer to the customer. These technological advancements have, similar to Panton, enabled the production of a new generation of product design. This research focuses on personalised objects which can be individually customised in relation to people's fo rms. 


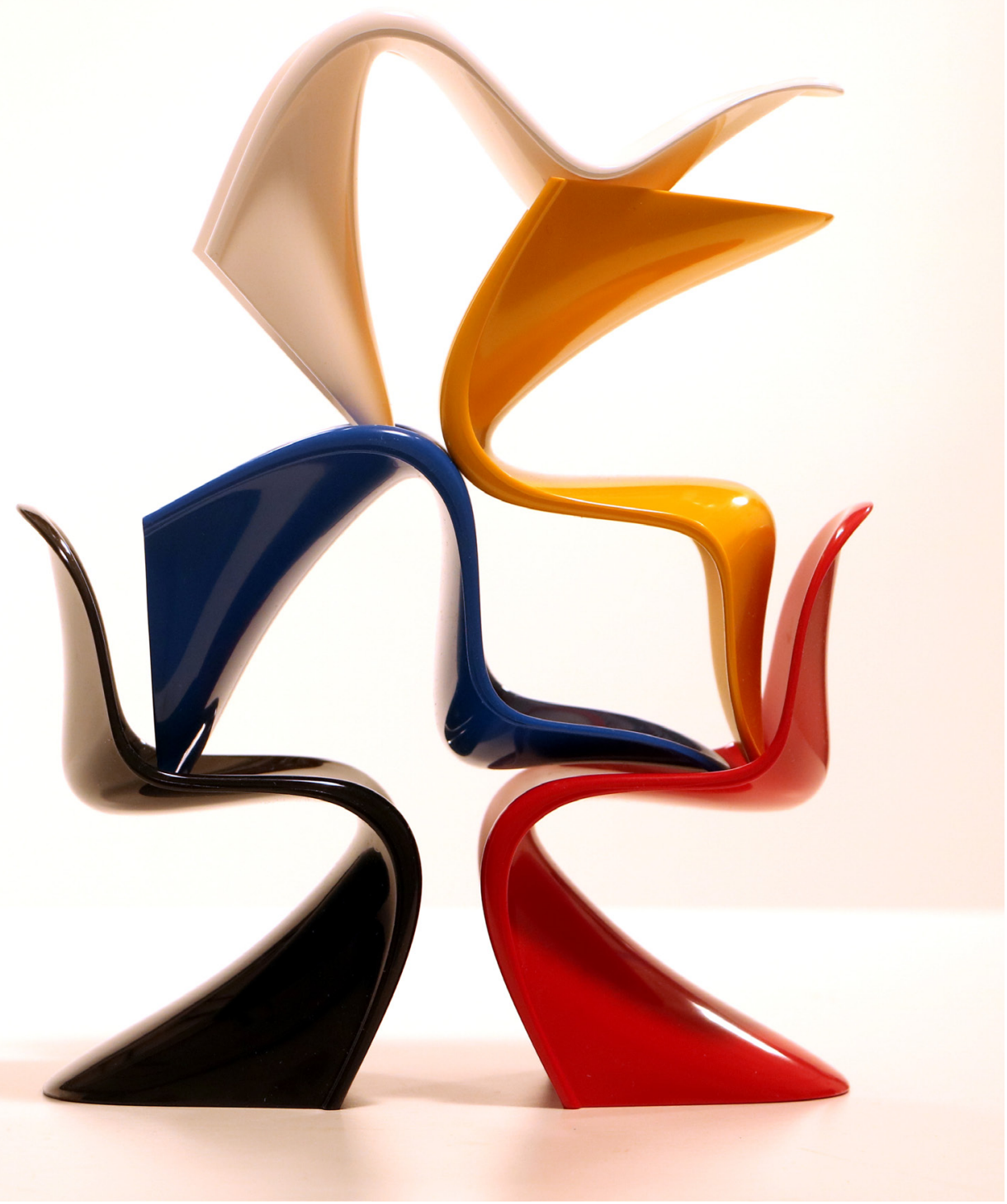

Figure 3 - Verner Panton's 'Panton Chair'. 


\section{Traditional 3D Printing}

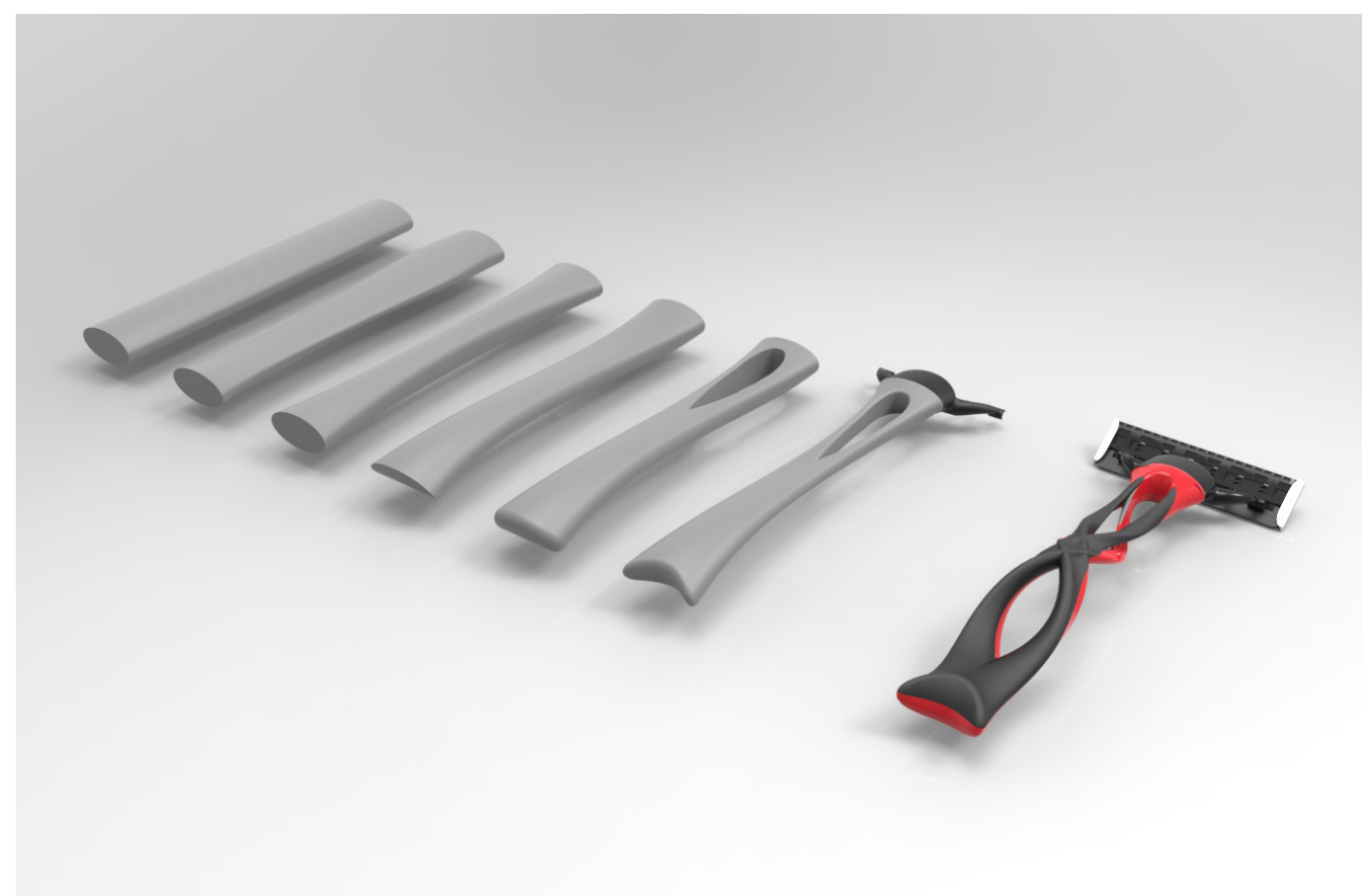

Example by the Author of the prototyping process used to design a new razor handle.

3D printing originated as a prototyping technology used to three-dimensionally build test objects as part of the developmental design process. It is a fast form of manufacturing that allows small product runs at relatively low cost. This enables rapid, rigorous prototyping and testing without the high costs other manufacturing methods would entail.

Daniel Lentz, lead industrial designer for Giant Bicycles, says "that the SLS printed seat parts simulate the final product. We modify the model, print, and take the SLS prototypes out for a ride, allowing us to feel and test the final product" ("Giant Bicycles begins 3D printing saddles", 2013). This traditional method of 3D printing is a critical step in Giant's design process. This enables the seating design to be perfected before it goes into mass production where a simple mistake could cost the company a large amount of money. 


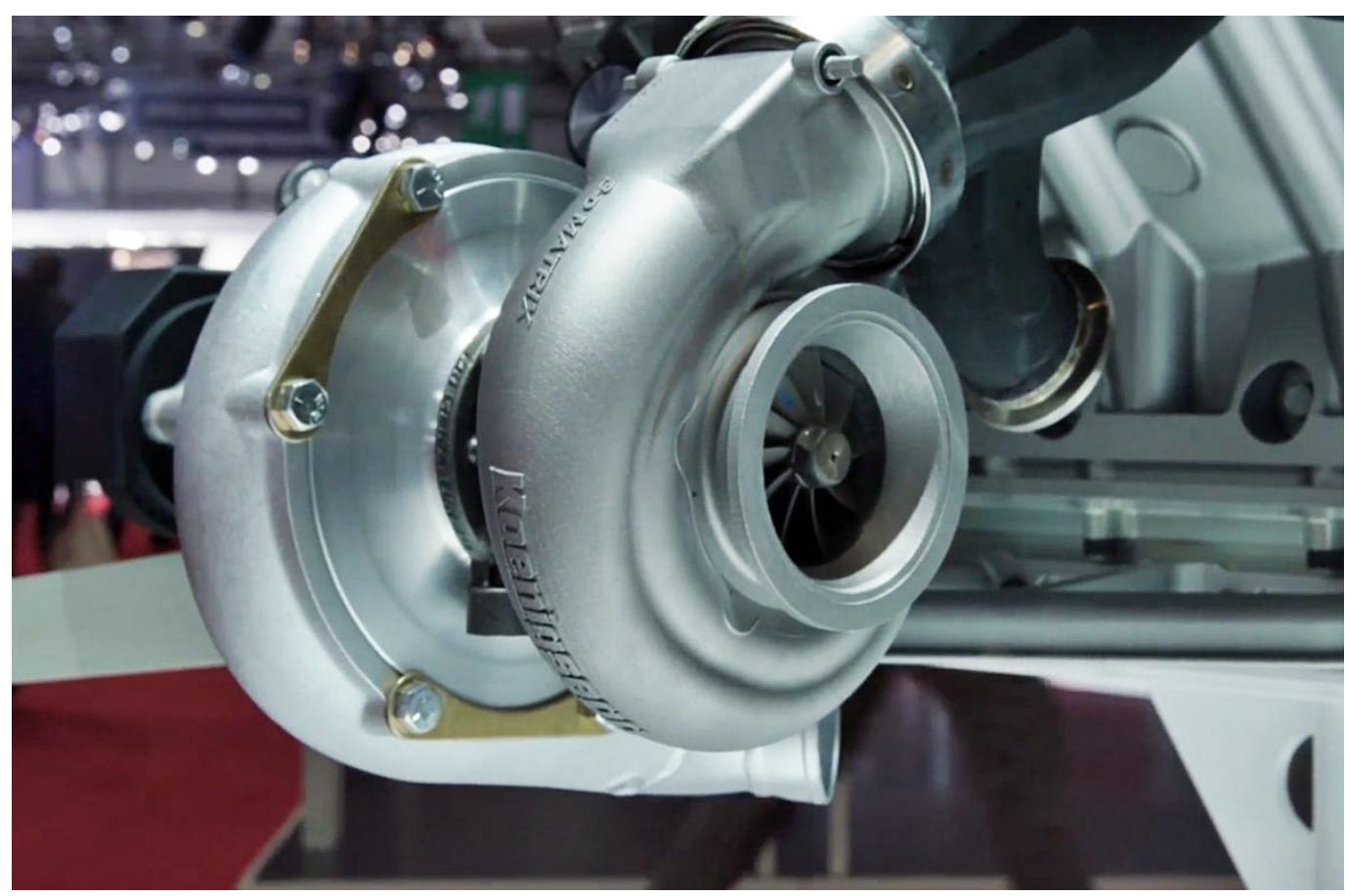

Figure 4 - Titanium 3D printed variable turbo.

With the continuous development of technology and materials, 3D printers can now manufacture models to a high enough quality they can be sold directly to the customer. Koenigsegg is a car manufacturer in Sweden that manufactures small numbers of high-performance super cars. Koenigsegg CEO and founder Christian von Koenigsegg explains "how the company takes advantage of 3D scanning and 3D printing technology to ensure its cars are lightweight and as advanced as possible" ("Inside Koenigsegg looks at 3D printing parts for world's fastest car", 2014). This company uses 3D printing to manufacture final parts on their most recent and most powerful car, the Koenigsegg One:1. They have used this technology to build a "variable turbo, providing the perfect boost for any RPM level (Figure 4). Entirely 3D printed, with moving parts printed within the enclosed chamber, this company takes advantage of 3D printing, building an object that could not be built any other way ("The World's First 3D Printed Turbo", 2014).

Vehicle components have traditionally been manufactured individually and assembled into functional parts, but Koenigsegg has innovatively removed this assembly process within the build of their turbos. These are modelled, built and removed from the printer fully functional. Similar to Panton's aspirations to build 'beyond belief' cantilevered seating, Koenigsegg's 3D printed titanium turbo proves that 3D printing technology is not only the next step in automotive performance, but also in the functionality of every day products. 


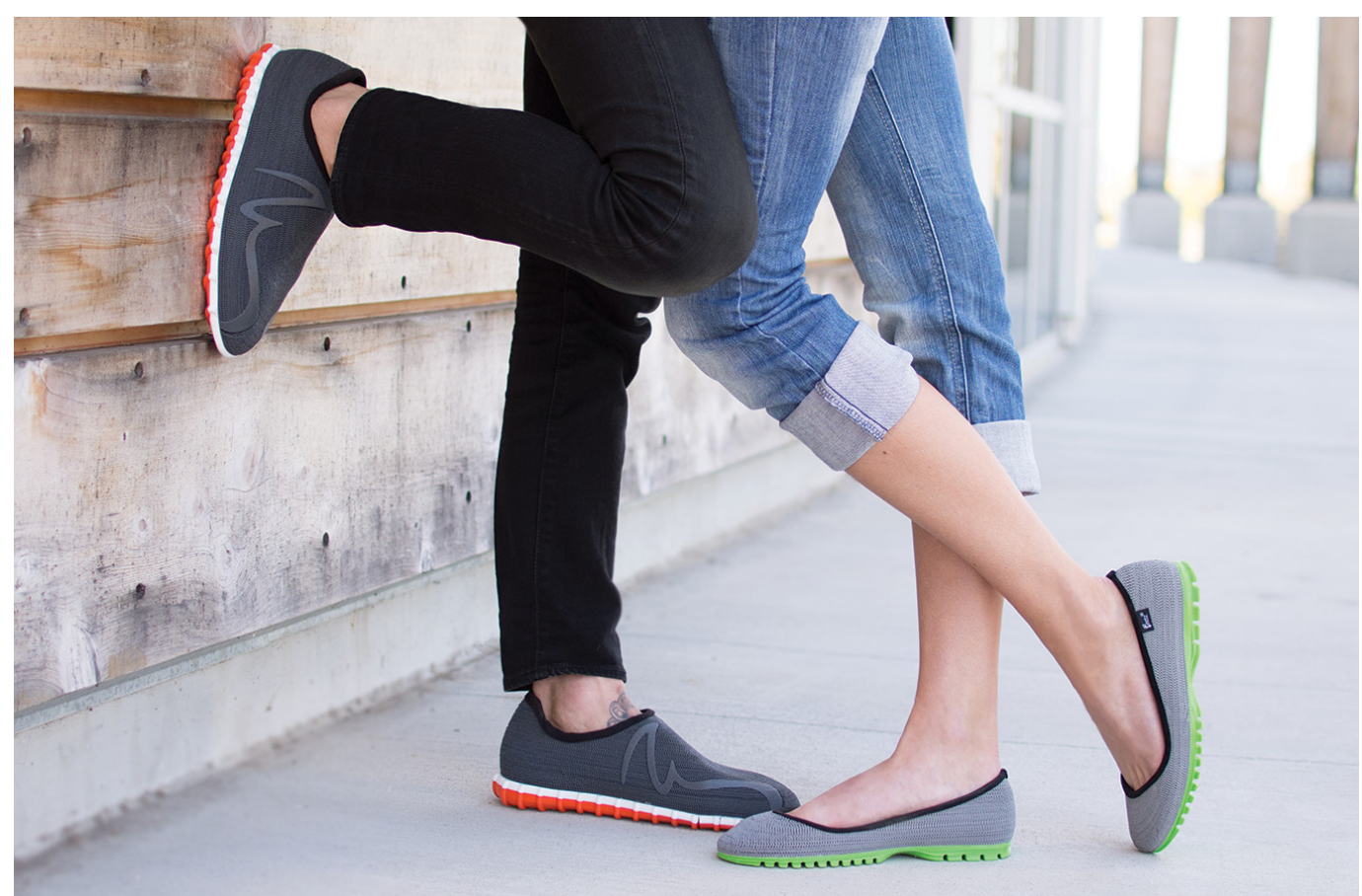

Figure 5 - Feetz custom 3D printed shoe (Reproduced with permission).

The shoe company Feetz, uses FDM 3D printing to create customised shoes specially shaped to individual customers' feet (Figure 5). Feetz was born when Lucy, the founder, was looking for that perfect pair of shoes to go with her new outfit, and five pairs in a row wouldn't fit ("Feetz Shoes", n.d.). The struggle of being unable to find shoes that fit her feet properly became a design opportunity where 3D printing was a cost effective option for the 'mass customisation' of footwear. Figure 6 clearly demonstrates FDM 3D printing is a real-world, viable solution when producing mass customised goods on a large scale (Gustke, 2016). The shoes are built from Thermoplastic Polyurethane (TPU) a flexible plastic, making this precedent significant to this research. Feetz has taken advantage of the digital code controlling the printer's movements which has allowed the shoes to be built with a breathable outer texture (figure 7).

Printing an object at a high enough quality that the object can be put into a vehicle or custom fitted to a client's feet are new concepts within the product industry. With the development of this technology and materials, the quality of $3 D$ printing will continue to grow to allow more opportunities for objects to be built this way inexpensively and efficiently. 


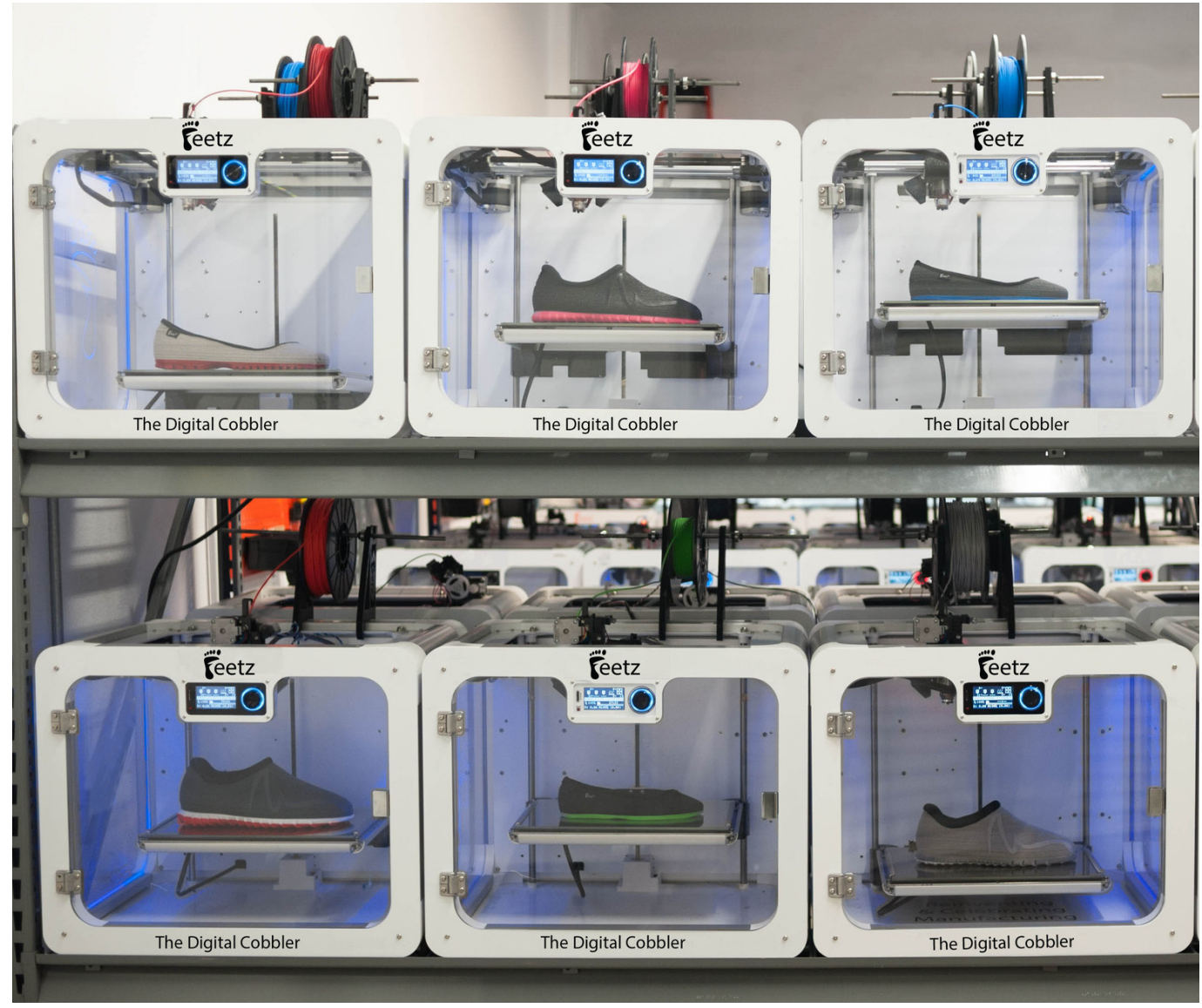

Figure 6 - Feetz 3D printing production line (Reproduced with permission).

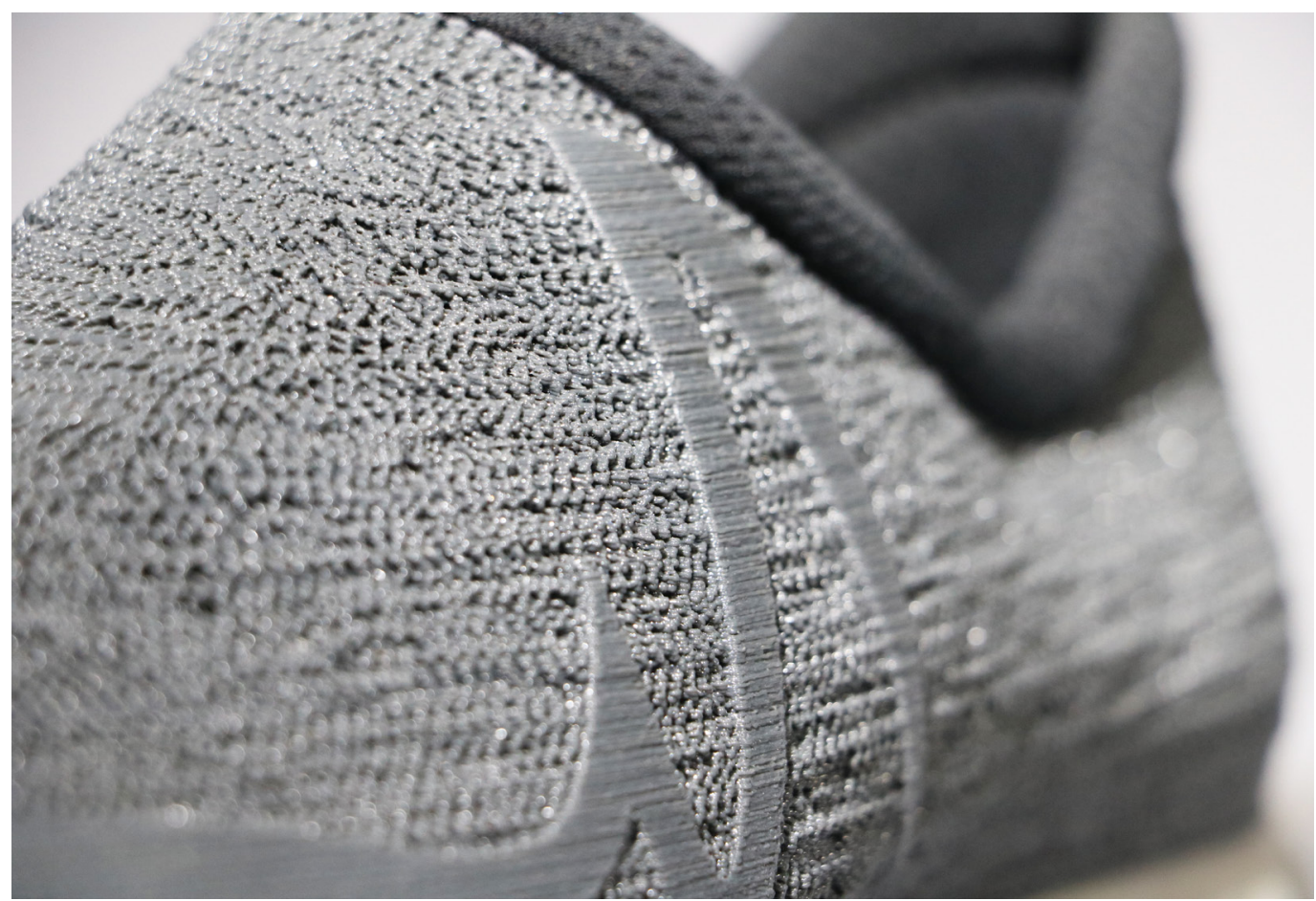

Figure 7 - Feetz 3D printed breathable texture. 
Mass manufacturing has larger production runs than mass customisation. This is due to the increased set-up time and number of tool changes raising the individual price of each object. This implies the larger run of units produced, the lower the cost becomes. It is due to the substantial tooling costs associated with traditional mass manufacturing that the world is full of generically shaped objects. While still fulfilling most people's needs, these machines allow minimal customisation or personal fit (Hopkinson \& Dicknes, 2003).

In a world developed around mass manufacturing universally shaped objects, unique and customised objects have become scarce but are desired.

3D printing has become a more viable solution for large scale mass customisation. Every 3D file that is sent to the printer can be different (Post, 2015). 3D printing digital files allows the objects, once printed, to be physically tested, evaluated, and re-designed. This allows the products being built to be more efficient and affordable while opening opportunities to having objects personally made to fit individual customer's bodies.

\section{Dual-Material 3D Printing}

Current seating design, for example, car seating, has multiple different densities of foam built into the base and back providing various support across the different parts of the seat (Gyi \& Porter, 1999). Using dual-material 3D printing, areas in a 3D printed seat can be made from the hard or soft material. This plays a significant role in adjusting the amount of support and comfort the seat provides to the body. Zaha Hadid, a British architect, found that by printing in different densities of material allowed her to "optimise the performance of key structural areas" (Howarth, 2014) creating a range of movement and textural properties. Hadid believes that this type of multi-property printing will form the bridge between prototyping and mass production of 3D printing. Unlike the connex3 3D printer, a highly expensive dual-material printing option used by Hadid, FDM 3D printing can take advantage of the multi-property aspects while offering more affordability. 


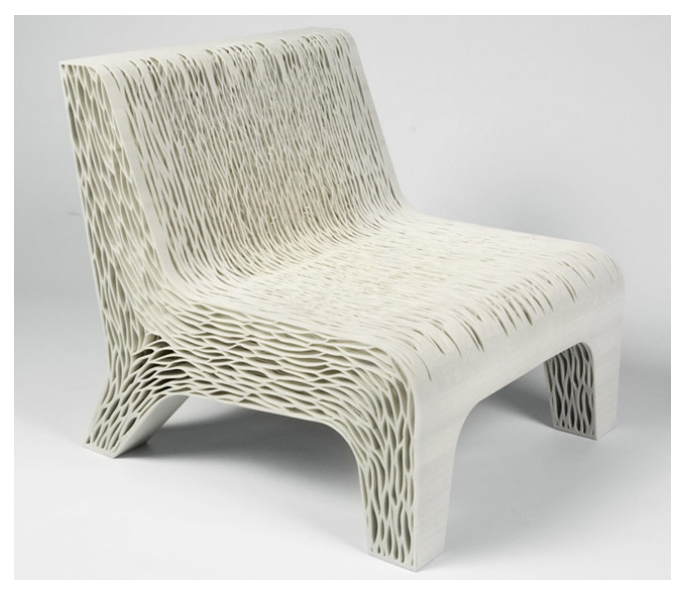

Figure 8 - Lilian Van Daal's Bio-mimicry chair.

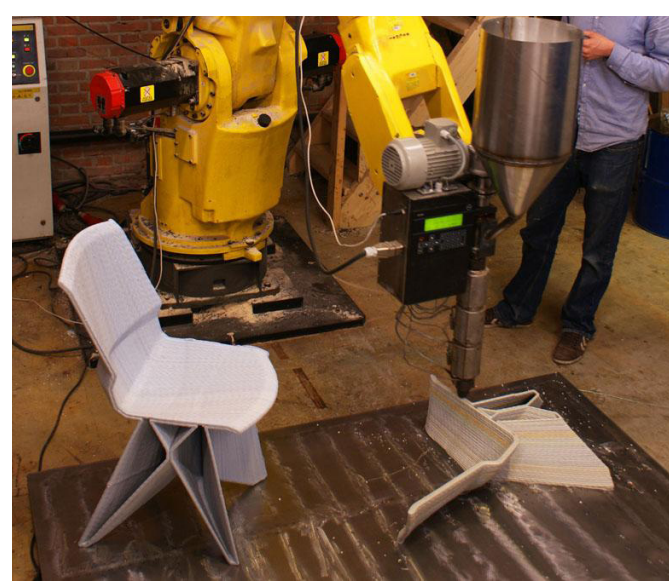

Figure 9 - Dirk Vander Kooij endless chairs.

Lilian van Daal's '3D printed bio mimicry chair' has its padding 3D printed within the model (Figure 8). Van Daal claims 3D printing can replace traditional upholstery techniques to produce spongy surfaced furniture (Hobson, 2015). Van Daal argues that with traditional upholstery manufacturing many different processes and tools are needed making it expensive and inefficient, whereas by using $3 \mathrm{D}$ printing it can all be done in one place using one machine.

Dirk Vander Kooij, a Dutch designer, uses the lines and movements of the manufacturing process in his work to create a unique, low-resolution aesthetic. Kooij's Endless Chairs series are built from recycled plastic using a similar method to FDM 3D printing where "melted recycled plastic is squeezed through a large nozzle" (Figure 9) (Johnston, 2015, p. 242). Within this 1:1 scale seating the thick bead of low-cost, recycled plastic emphasises the layer lines creating patterns within his work. Kooij's work displays how recent developments in printing materials have enabled 3D printers to build objects with the quality and accuracy comparable to traditionally manufactured items (Schubert, Langeveld, \& Donoso, 2013).

Both of these projects take advantage of the 3D printing method employed to build objects at human scale. By using full-scale 3D printing, the high costs affiliated with the construction of moulds used for injection moulding has been eliminated.

Rapid 3D printing developments now allow prints to be higher quality, more economical, and more efficiently manufactured, moving the customisable 3D printed product straight to the customer. 3D scanning is one method of capturing the complex digital form used in the design of personalised 3D printed objects. 
Anthropometric variation in the human body is important to this research allowing products to fit the form of people. 3D scanning entails digitally capturing a complex physical form as a three dimensional CAD file.

Koenigsegg, the supercar manufacturer, uses "3D laser scanning technology to scan the shape of the prototype car seat, which is edited using computer software perfecting the seats surface and shape" ("Inside Koenigsegg looks at 3D printing parts for world's fastest car", 2014). This is an efficient way to make a physical object digital. Sophie Kahn, a New York based digital artist uses "a hand-held 3D laser scanner" (Johnston, 2015, p. 129) in her work to explore expression and emotion by purposely miss-stitching together scans of the female body (Figure 10). Textures and geometry are captured using a handheld 3D scanner producing high definition digital files which are later used to produce the physical sculpture.

Custom moulded seating shaped to individual people's bodies is used within the sports industry in applications where the user requires support. In Formula One, a mould of the driver's body is created using expanding foam (unknown, 2013). This creates a shell which is 3D scanned allowing a digital seat to be created to the driver's exact shape. Similarly Paralympic basketball wheelchair seats are moulded to the individual players form. Players and coaching staff were surveyed about their existing wheelchair designs, and their opinions highlighted "an opportunity to optimise performance by introducing a fully customised seat for each player" (Morby, 2016). Thermoformed moulds were made and then 3D scanned digitally creating customised seating for each individual player. This was not to increase comfort but to reduce weight and allow less movement between the person and the seat. This kind of technology is valuable within sports where they are prepared to sacrifice players comfort to enhance performance. If a piece of everyday seating was shaped to fit the body, it would provide extra comfort and support perhaps revolutionising the way seating is designed.

The number of ways in which 3D scanning technologies are used to capture 3D geometry is increasing. Although all of these projects demonstrate 3D scanning in unique ways, they are each valuable examples of how this technology is professionally used. Whether it is used to aid the design process, increase performance of sports teams, or as a tool to capture living art, these different approaches have taken advantage of the ease in digitally replicating highly detailed physical objects.

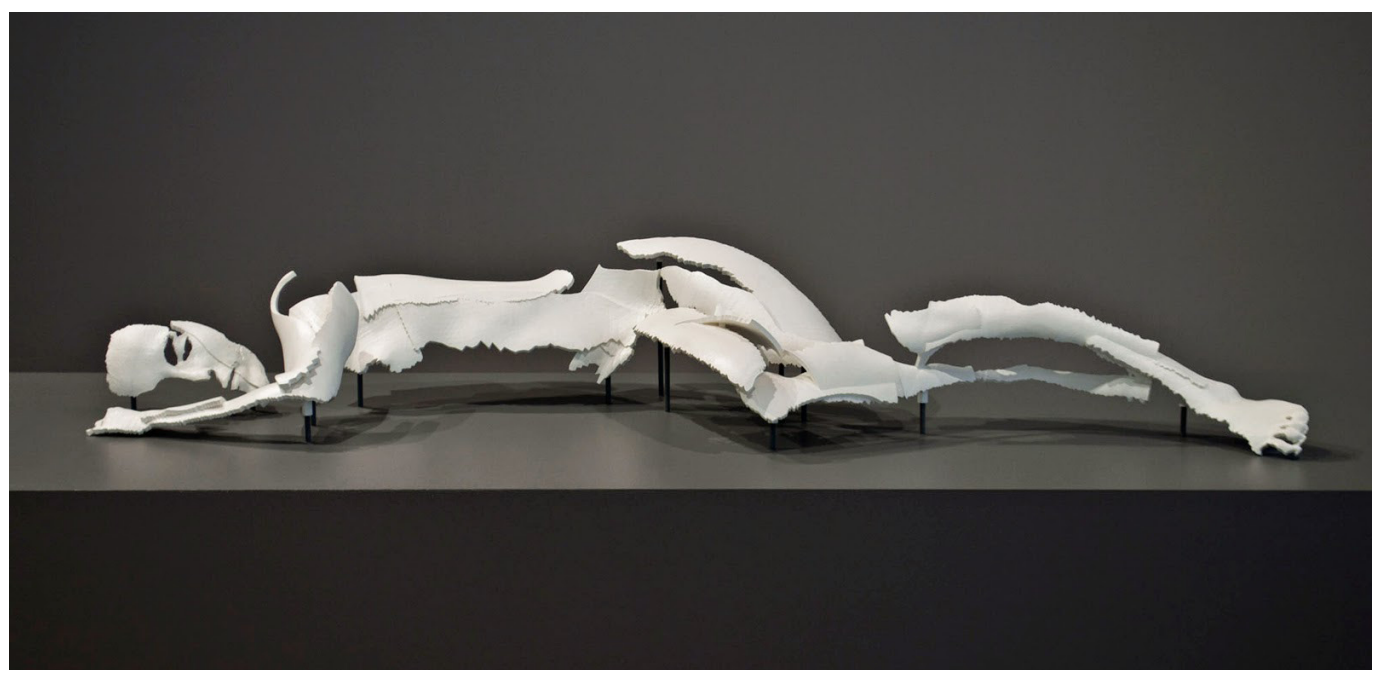

Figure 10 - Sohpie Kahn's work explores expression and emotion. (Reproduced with permission) 
Having a seat that is designed and shaped to fit an individual is a specialised idea that has been introduced into areas of the sports industry but not everyday life.

Universal seats are designed for the broadest user group. Stock motorcycle seats are generally made up of three generic parts. A hard injection moulded base, foam padding, and a vinyl cover. These components are designed to be mass produced as fast and efficiently as possible. As a result the universal design of the seat fits the broad percentile of riders, however, is unable to cater for specific body proportions.

Figure 11 illustrates the data collated through a UK study set out to understand "the average physical dimensions of motorcycle riders with relevance to the design of motorcycles" (Robertson \& Minter, 1996. p. 223). Height, leg lengths, arm lengths and weights of 109 male and 103 female motorcycle riders were reviewed resulting in a proportion percentile which is above the normal average that products are generally designed for. Although this study uses a small sample size, it suggests the motorcycle design industry aims at designing motorcycles for riders with a height of $160-180 \mathrm{~cm}$ tall. Although this percentile includes the majority of people's body proportions, for the people outside this size range, the motorcycle is uncomfortable and awkward to ride.

Using 3D printing and 3D scanning this research explores refining the design of custom motorcycle seating to shape it to an individual riders form. This concept caters for every bodies size and proportion.

\section{PARTICIPANT STATURE}

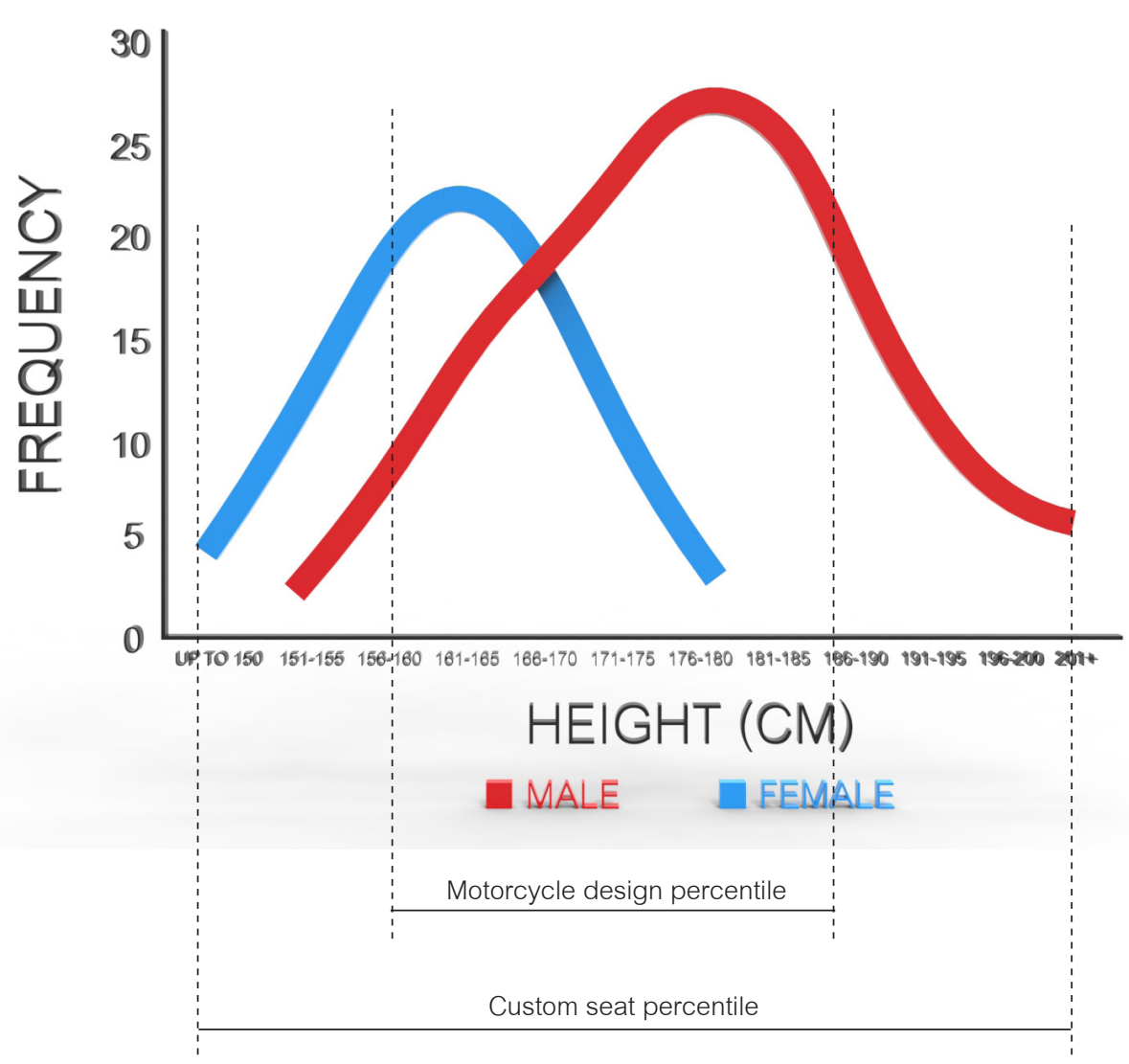

Figure 11 - The average physical dimensions of UK motorcycle riders. 



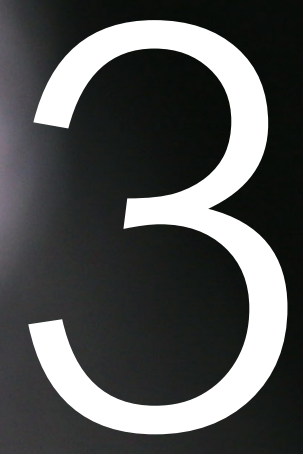

METHODOLOGY 


\section{METHODOLOGY}

\section{Research Through Design}

Research through design (RTD) is a methodology referred to within the creation of a product, service, environment, or system (Zimmerman, J., Stolterman, E., \& Forlizzi, J. 2010). It is through this making process new knowledge is created, systematically refining physical and theoretical ideas in order to fulfil an objective. William Gaver explains that "designers often turn to existing examples of design to inform the development of their own ideas" (2012, p.943).

Defined for this research, RTD is used as a design process focused on refining the relationship created between body and object. On top of theoretical knowledge, this process incorporates the design procedure of concept, development, iteration, and refinement. Figure 12 illustrates how this system is used during the developmental design process when refining a prototype to a final product.

RTD includes material research, development work, and the critical act of recording and communicating the steps, experiments, and interactions of the design process (Martin \& Hanington, 2012). This not only provides the researcher with insights into the inner workings of the design itself but also the manufacturing processes and materials involved in the creation of the object. One of the most important things RTD provides to the design process are the faults. "The artefacts produced in this type of research become design exemplars" (Zimmerman et al. 2007, p. 493), revealing large or small problems which are solved to iterate and develop a product. 


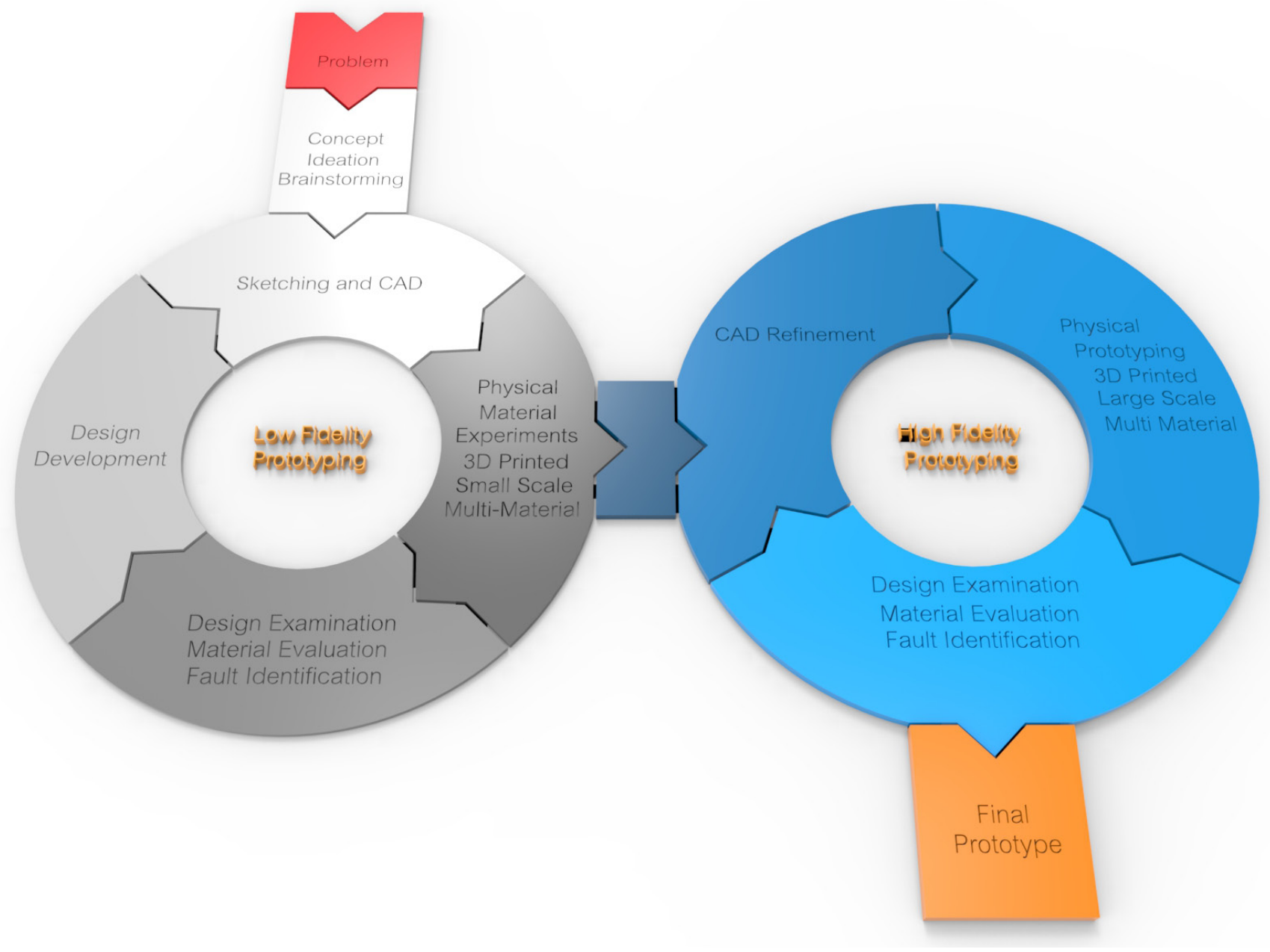

Figure 12 - The RTD Structure that will be followed through this research. 


\section{Research Methods}

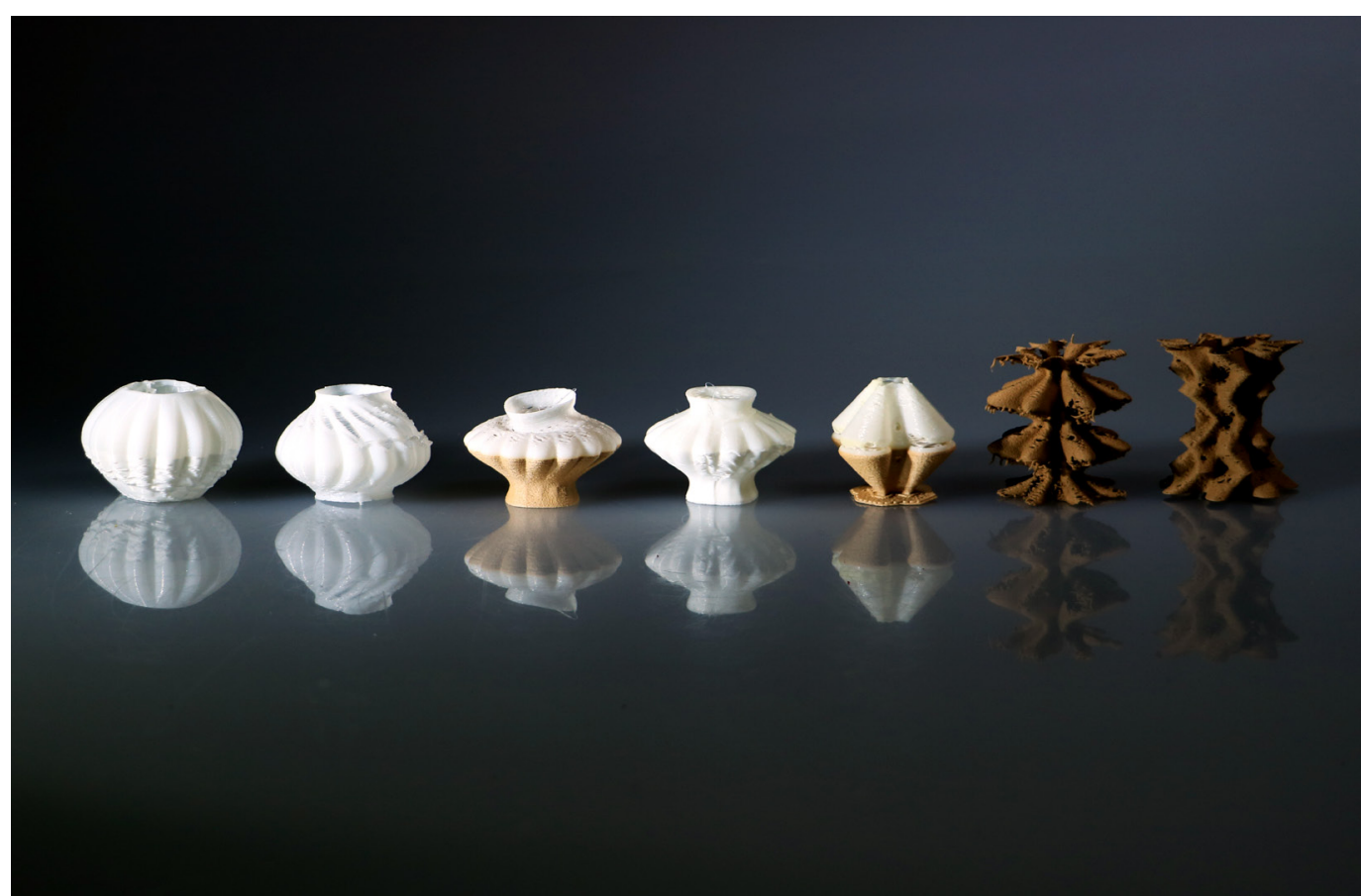

3D printed material experiments illustrating the developement of draft angles.

\section{PROTOTYPES}

Prototypes are the physical realisation of a product or interface concept. This represents the translation of concepts and ideas into tangible form, allowing the testing and evaluation of physical concepts (Martin et al., 2012). This research begins by creating many low fidelity prototypes which allows for testing of materials and 3D printing technologies. This process gives timely feedback and constructive evaluation encouraging developmental changes. Once an insight is gained into the materials and manufacturing, high fidelity prototypes are used to create more realistic models which more accurately represent the final prototype.

Additive manufacturing is revolutionising the way we produce and build products. Due to media exposure, the capability of 3D printing technologies and materials is rapidly developing. Different sized FDM printers with multiple extrusion heads are becoming increasingly popular offering larger, more complex printing capabilities. 


\section{D PRINTING}

There are many methods of additive manufacturing that come beneath the broader title of $3 \mathrm{D}$ printing. Four popular methods of 3D printing used to fuse plastics together are SLA, DLP, SLS, and FDM 3D printing.

- Stereolithography (SLA) 3D printing uses "an ultraviolet laser, systematically solidifying a liquid photopolymer resin" (David Bak, 2003, p.343) layer by layer three-dimensionally building the model. This method of 3D printing creates highly detailed models. However it is expensive which limits it to smaller objects.

- Digital light processing (DLP) 3D printing uses a similar process to SLA. A light projector displays and solidifies sections of the CAD model which causes resin to cure layer by layer building the 3D model (Bogue, 2013). Adidas utilised this method of 3D printing to create the mid-sole for the Futurecraft shoe (Figure 13). Using this method of manufacturing to build the shoe soles creates opportunities for small product runs tailored or moulded to suit an individual's foot-shape (Vincent, 2017). Currently DLP 3D printing offers high definition, mono-material $3 \mathrm{D}$ printing at a high price reducing it to smaller scales.

- Selective laser sintering (SLS) 3D printing uses a laser to accurately sinter plastic powder together layer by layer building the 3D model (David Bak, 2003). This method has a large variety of material options as well as a range of scales. Although this option has low costs, at this stage it is limited to single material printing.

- Fused deposition modelling (FDM) 3D printing provides a low entry cost to manufacture. A $3 D$ object is designed using CAD software, the $3 D$ data is sent to the $3 D$ printer, and the mono material FDM 3D printer extrudes a bead of molten plastic onto the print bed. Layer by layer the object is built out of the plastic, creating low-cost product, at an efficient rate (Figure 14 \& 15). Dual-material FDM 3D printing is a slightly different process where there are two extrusion nozzles and multiple materials (Figure 16).

Although FDM 3D printing may not be able to produce highly defined models such as those of SLA, DLP or SLS printing, it is economically suitable and offers a range of scales as well as dualmaterial usage. It is the method which is used in this research.

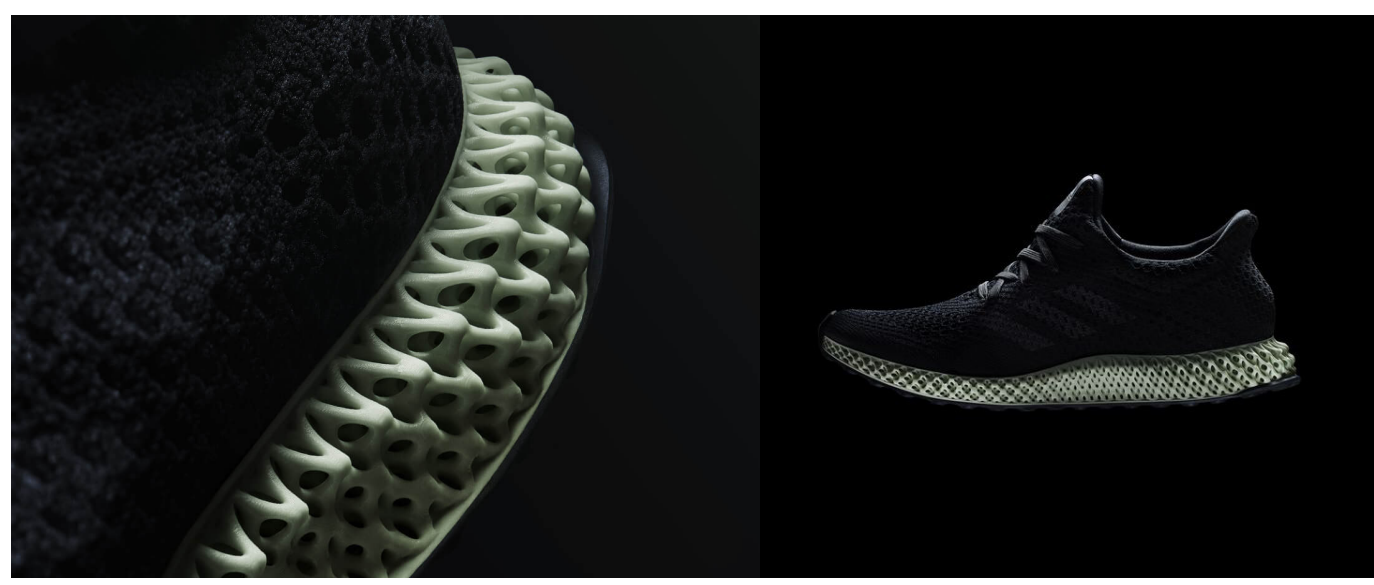

Figure 13 - Adidas Futurecraft DLP 3D printed shoe. 


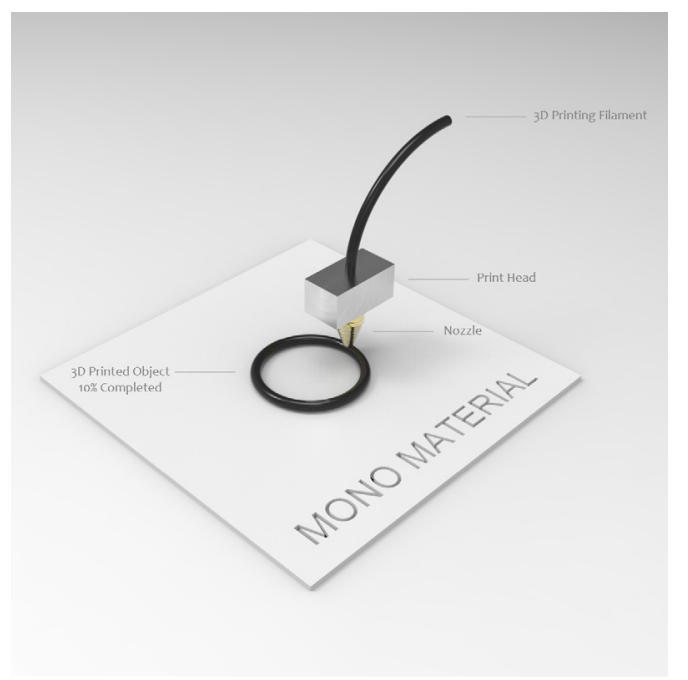

Figure 14 - Mono Material FDM 3D printing.

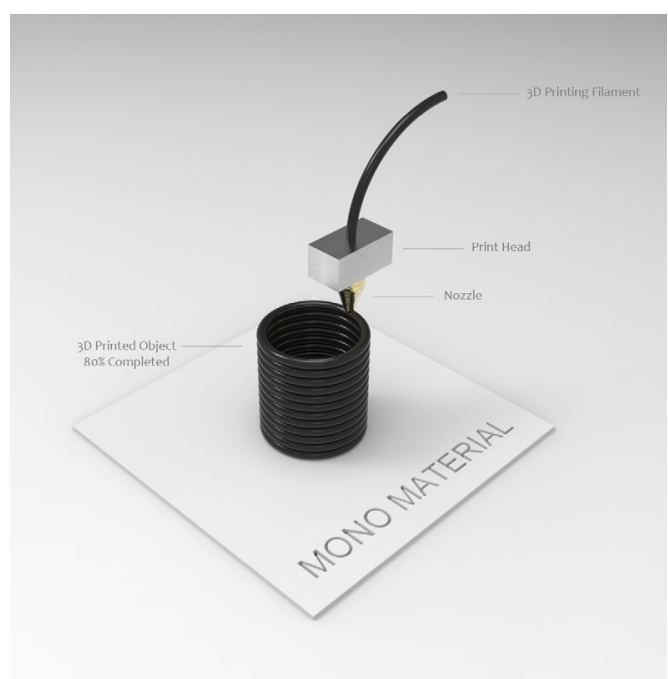

Figure 15 - Mono Material FDM 3D printing

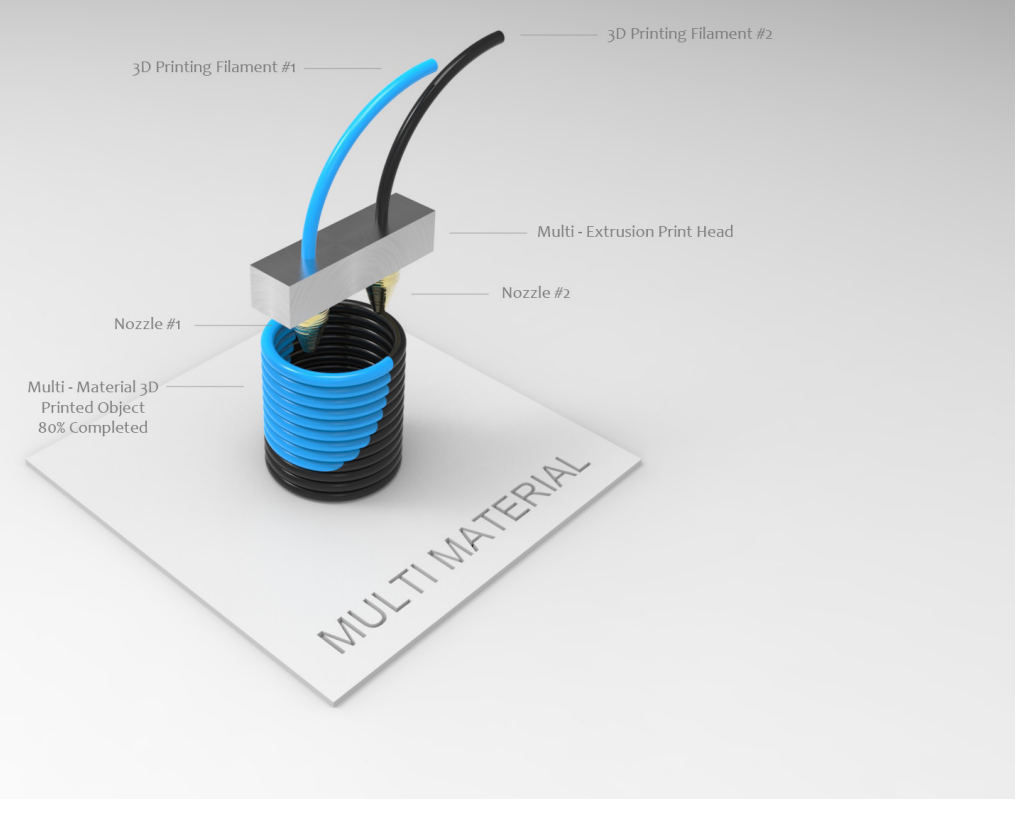

Figure 16 - Multi material FDM 3D printing. 


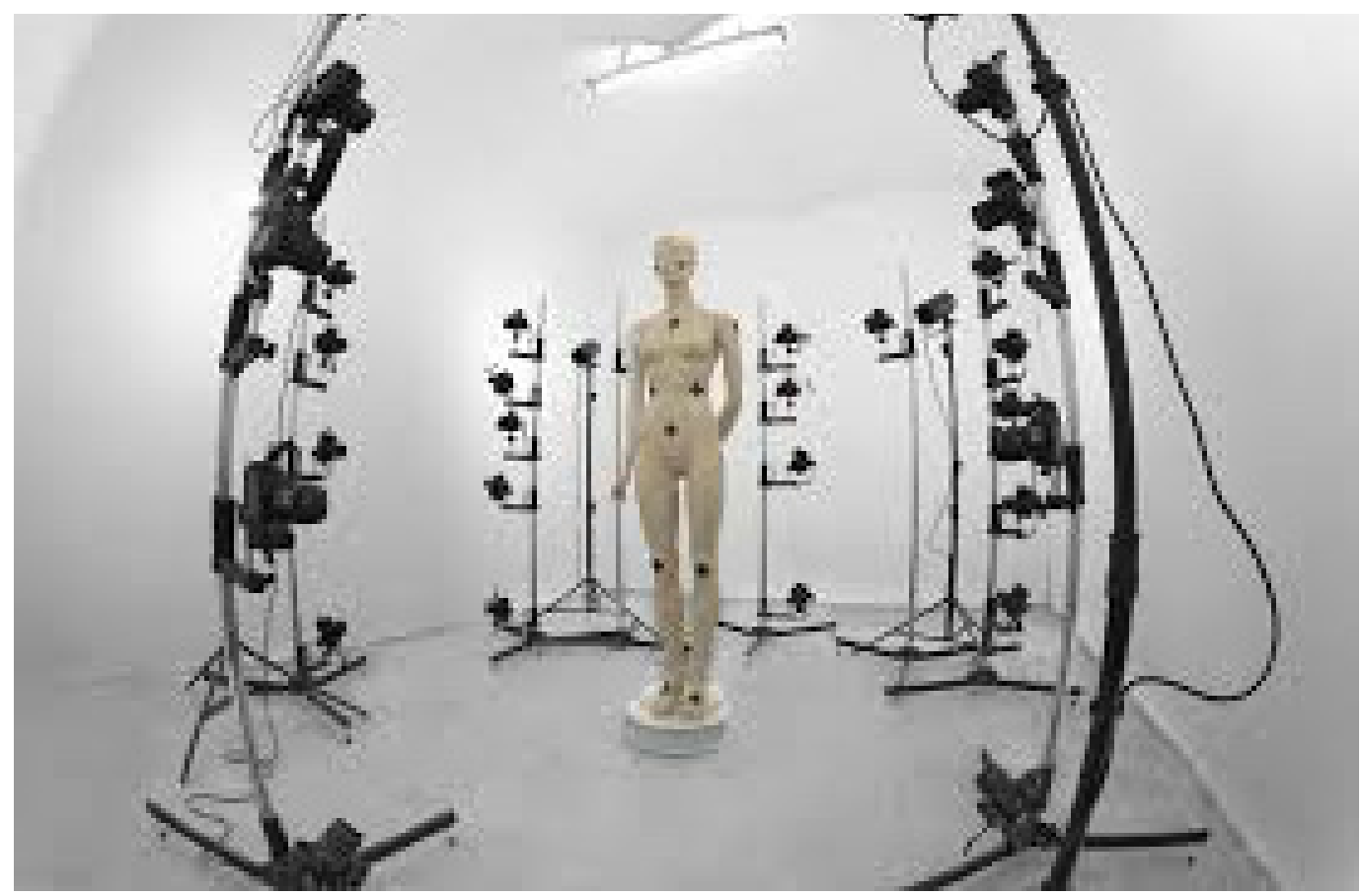

Figure 17 - Photogrammetry studio (Reproduced with permission).

Two popular methods of capturing 3D scanned data is through photogrammetry and Structured Light Scanners (SLS). Photogrammetry is a method of scanning where the object is captured using still images from every angle securing the digital form. These photos are inputted into "a piece of software that uses the colours, shadows, and textures as benchmarks to re-attach them to a three-dimensional volume" (Bernier, Luyt, \& Reinhard, 2014, p. 29). There are multiple ways of capturing the photos used for this method.

- One method of photogrammetry uses a "system that can trigger several cameras at once" (Bernier et al., 2014, p. 29) with the scanned model sitting in the middle of all the cameras. Using many SLR cameras set up in a spherical shaped room allows many photos to be captured instantaneously (Figure 17). Karin Sander used this method to scan people for one of her fine art exhibitions. She then 3D printed 1:10 scale models of the participants using the 3D scanned forms as a way of exploring human physicality. (Itoskins, 2014). The combination of FDM 3D printing and 3D scanning have been coupled in this project to create unique objects which would not have been possible using traditional mass manufacturing methods.

- Another method of photogrammetry is by using a single external camera. Walking around the model "taking photos of the object from all possible angles" (Bernier et al., 2014, p. 122) builds an archive of perspectives allowing the object to be stitched together using software to create the 3D model. This is an affordable method of 3D scanning however when capturing live models, this method is difficult to get a successful scan. Moving around the model taking photos will take time, and if the live model moves the final 3D scan may compile incorrectly or blur. 


\section{D Scanning - Structured Light Scanner (SLS)}

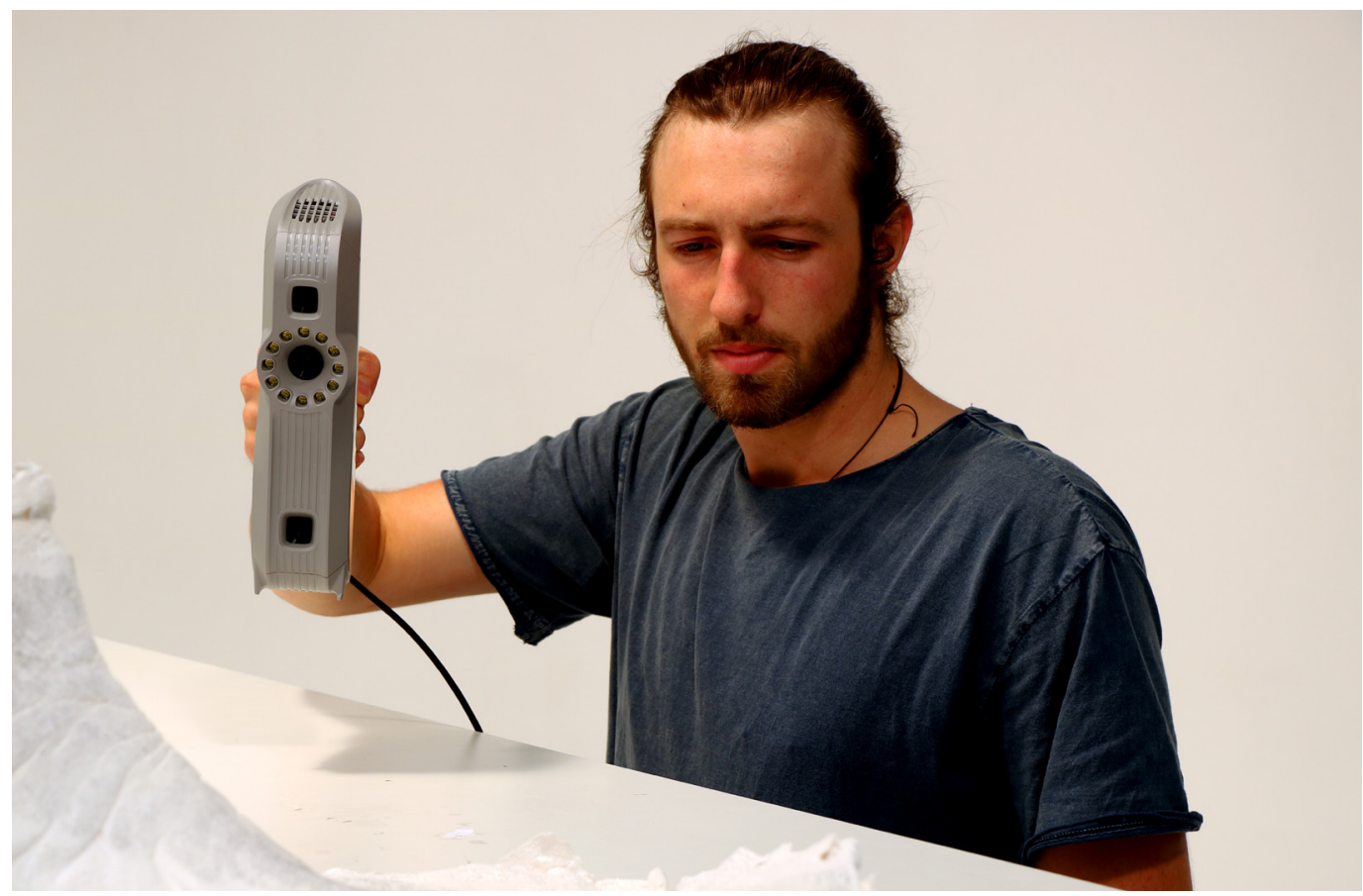

The Artec 3D scanner.

The second method of 3D scanning is using Structured Light Scanners (SLS). SLS scanners use "a video projector that sweeps the surface of an object while blasting it with patterns, stripes, or grids" (Bernier et al., 2014, p. 28). It is this reflecting light or pattern that the 3D scanner uses to measure the deformation of the object, three-dimensionally mapping its surface.

This research uses the Artec EVA 3D scanner to capture the forms of the participants. The scanners real-time feature allows the user to view the 3D scan live on the computer screen. This visually displays the digital data as it moves over the object being scanned. This is helpful to the user showing areas which have been missed or need re-scanning. This makes the 3D scanning process efficient, and timely. 


\section{Materials}

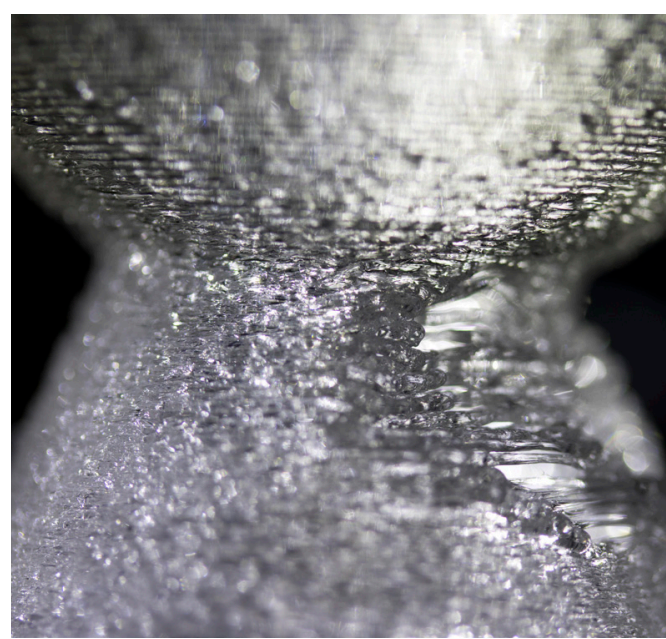

Above - TPU plastic

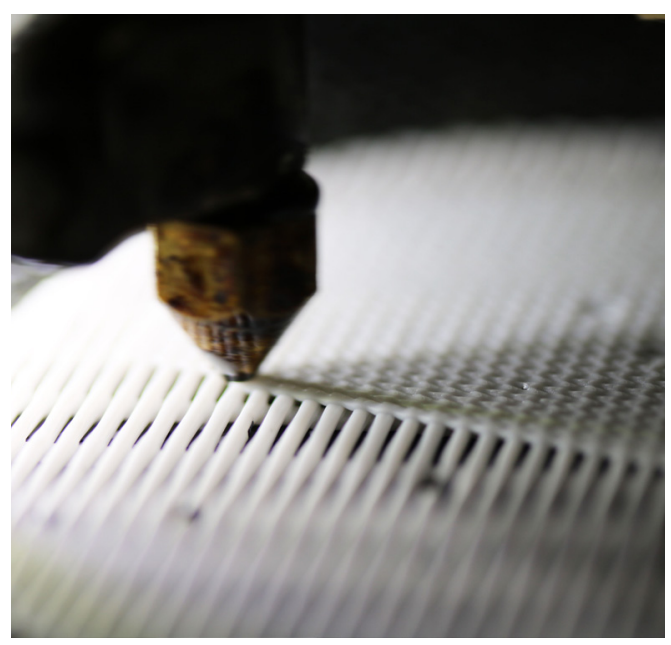

Above - PLA plastic

The FDM 3D printing materials used within this project are Thermoplastic Polyurethane (TPU) and Polylactic Acid (PLA).

- $\quad$ The TPU plastic is a strong and flexible material. The tensile strength of TPU plastic is robust and durable while allowing a range of flexibility and resilience (Seidenstücker \& Fritz, 1998).

- $\quad$ PLA plastic is a biodegradable thermoplastic which is hard and rigid (Ochi, 2008).

Using dual-material FDM 3D printing allows for a 3D printed object to be made up of combinations of materials. Using a dual-material 3D printer, these two materials can be selectively placed within a single print creating strength, rigidity and movement in the specific areas desired. 


\section{Equipment}

- Up Mini 3D Printer - Small scale FDM 3D printer. Capacity of $100 \mathrm{~mm} \times 100 \mathrm{~mm} \times$ $100 \mathrm{~mm}$

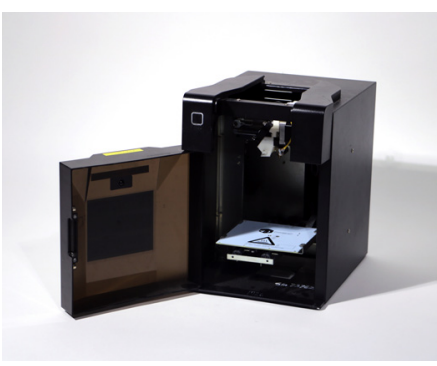

- Up Box 3D Printer - Medium scale FDM 3 D printer. Capacity of $260 \mathrm{~mm} \times 200 \mathrm{~mm}$ $\times 200 \mathrm{~mm}$

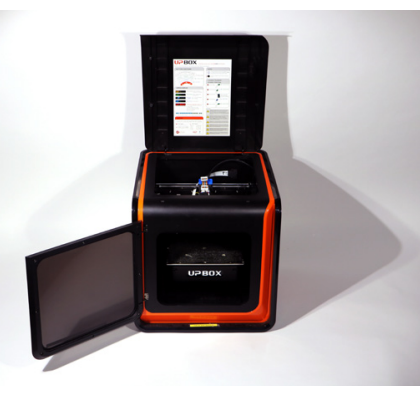

- $\quad$ Big Rep 3D Printer - Large scale FDM 3D printer. Capacity of $1 \mathrm{~m} \times 1 \mathrm{~m} \times 1 \mathrm{~m}$

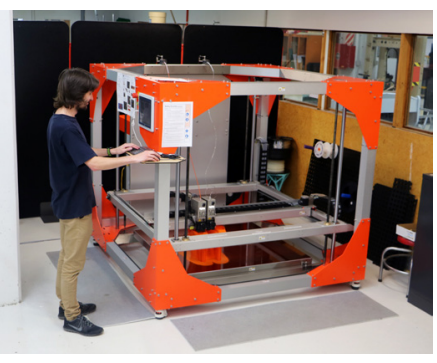

- Body pressure Measurement System (BPMS)

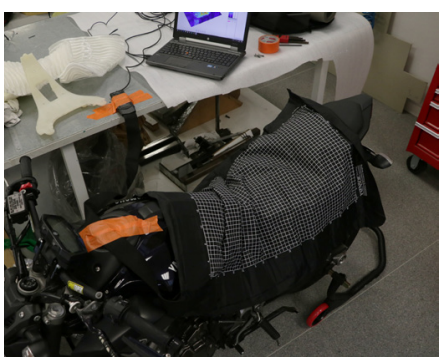

- $\quad$ Artec scanner - Structured Light Scanners (SLS) 3D scanner

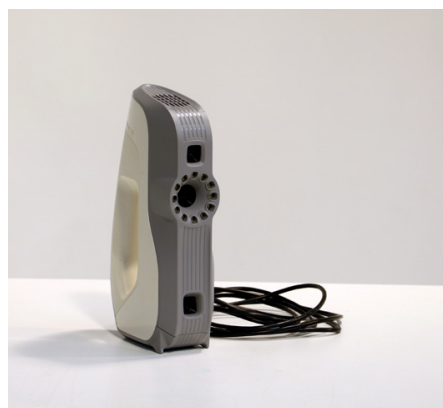




\section{DESIGN PROCESS}

\section{Introduction}

This chapter describes and illustrates the design process undertaken from the first small scale material experiments, to the final full scale, dual-material seat prototype. CAD modelling, high and low fidelity prototyping, rendering and photographic documentation are illustrated in this chapter. Alongside this visual narrative is a text commentary that describes the development emphasising critical decisions or change points. This also addresses the strengths and weaknesses of the experiments and the logic and reasoning behind each design iteration.

The scope of this research involves;

1. An initial exploration into materials and manufacturing technology at small scale

2. Defining an application

3. Designing high fidelity seat prototypes that are then evaluated.

Below is a detailed list of the components this chapter covers:

- $\quad$ Small scale, Mono and Multi-Material, and Low Fidelity Material Experiments

- $\quad$ Material Temperature Testing

- $\quad$ Draft Angle Testing

- Design Application Selection

- $\quad$ Support Experiments

- 3D Scanning

- $\quad$ Full Scale, Mono-Material, High Fidelity Seating Prototypes

- $\quad$ Large Scale Testing

- $\quad$ Large Scale, Dual-Material Experiments

- $\quad$ Final Full Scale, Dual-Material, High Fidelity Seat Prototype

- Post Processing

- Participant Evaluation

- $\quad$ Aesthetic Opportunities 
When designing an object to be FDM 3D printed, analysis must be carried out regarding the printer to achieve its optimum function. In particular with FDM 3D printing, an object can be designed to print as a single bead of material, the extrusion mechanism has less stopping and starting to do. This makes it more efficient for the printer to create the object. The chosen method of $3 D$ printing therefore has a direct correlation to the CAD designs used to form the models being printed.

Initial small scale, mono-material forms used both TPU flexible and PLA wood materials to explore the 3D printer settings. The settings control the speed the printer runs at, the build layer height of the printer, and the wall thickness.

Programming the printer to a default PLA plastic temperature set the print nozzle to $210^{\circ} \mathrm{C}$. This is the material manufacturer's temperature recommendation for both the PLA and TPU plastic. However the nozzle initially struggled to extrude both materials. Increasing the extrusion nozzle diameter from $0.4 \mathrm{~mm}$ to $0.8 \mathrm{~mm}$ allowed the material to flow through with less drag establishing successful extrusion.

Low fidelity material experiments use simple cylindrical shapes made with single and double wall layers allowing the focus to be on the material qualities, limiting erratic machine movements, reducing unnecessary flaws. 


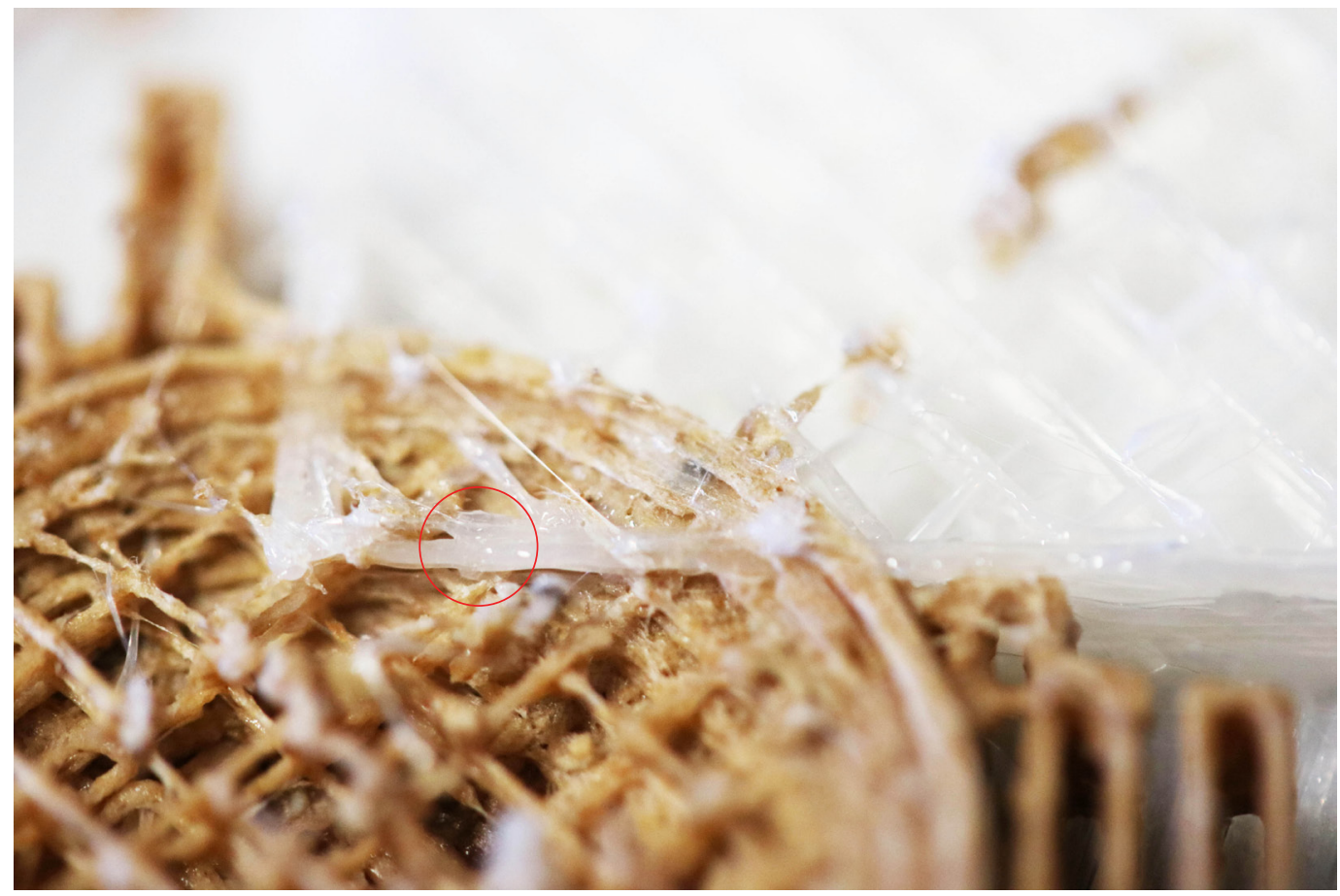

Continuous stopping and starting of material extrusion. This creates a build-up of plastic in the printed object.

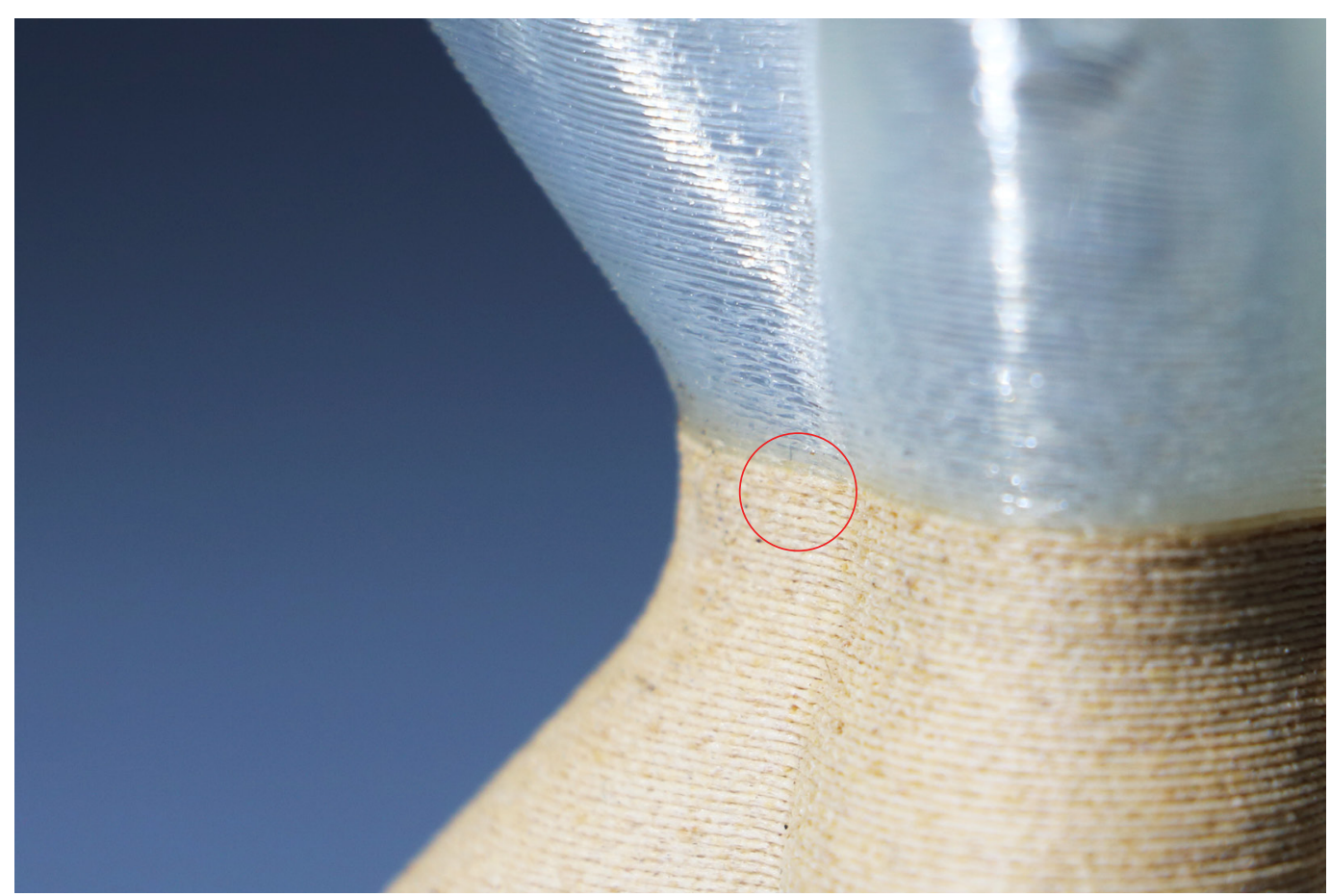

High quality material experiment designed to print as a single bead of material. 


\section{PRINTER SPEED}

- $\quad$ The 3D printer used for the low fidelity experiments is an Up Mini (see equipment, pg. 48). The printer has three default speed settings. Fast, Normal, and Slow. Having the printer run on the normal setting gave the most success with these small scale mono-material prints, creating fewer blockages, giving an even plastic extrusion.

\section{SET LAYER HEIGHT}

- The printer also has default layer height settings. The layer height can be set at $0.1 \mathrm{~mm}$, $0.15 \mathrm{~mm}, 0.2 \mathrm{~mm}, 0.25 \mathrm{~mm}, 0.3 \mathrm{~mm}, 0.35 \mathrm{~mm}$, and $0.4 \mathrm{~mm}$. These settings control not only the height of each printed layer but also the extrusion flow rate. Using the printer when set to a $0.3 \mathrm{~mm}$ layer height increased the flow rate of the material forcing a cleaner, more even bead of molten plastic through the nozzle. The lower the layer height, the higher the risk of the nozzle blocking.

\section{WALL THICKNESS}

- Controlling the wall layering allows for an exploration into the flexibility and rigidity of the PLA and TPU plastic. Printing with a single or double layered wall thickness gave various results. Printing both materials as a double layered wall gave high quality, clean prints. This is because there was more material for the new bead of extruded material to adhere to (Figure 18). When printing with a single wall, unlike the double layered wall thickness, the prints became holey and blotchy where the bead of plastic had not adhered to the previous layer. I considered this a design opportunity, rather than a problem, where the 3D printer had brought digital craft into the object by creating textures and patterns which were not designed into the CAD model.

Reflection.

Using these initial models to explore the 3D printer's settings has grown the researcher's essential knowledge around 3D printing. This knowledge builds an instinct about what makes the 3D printing process work best.

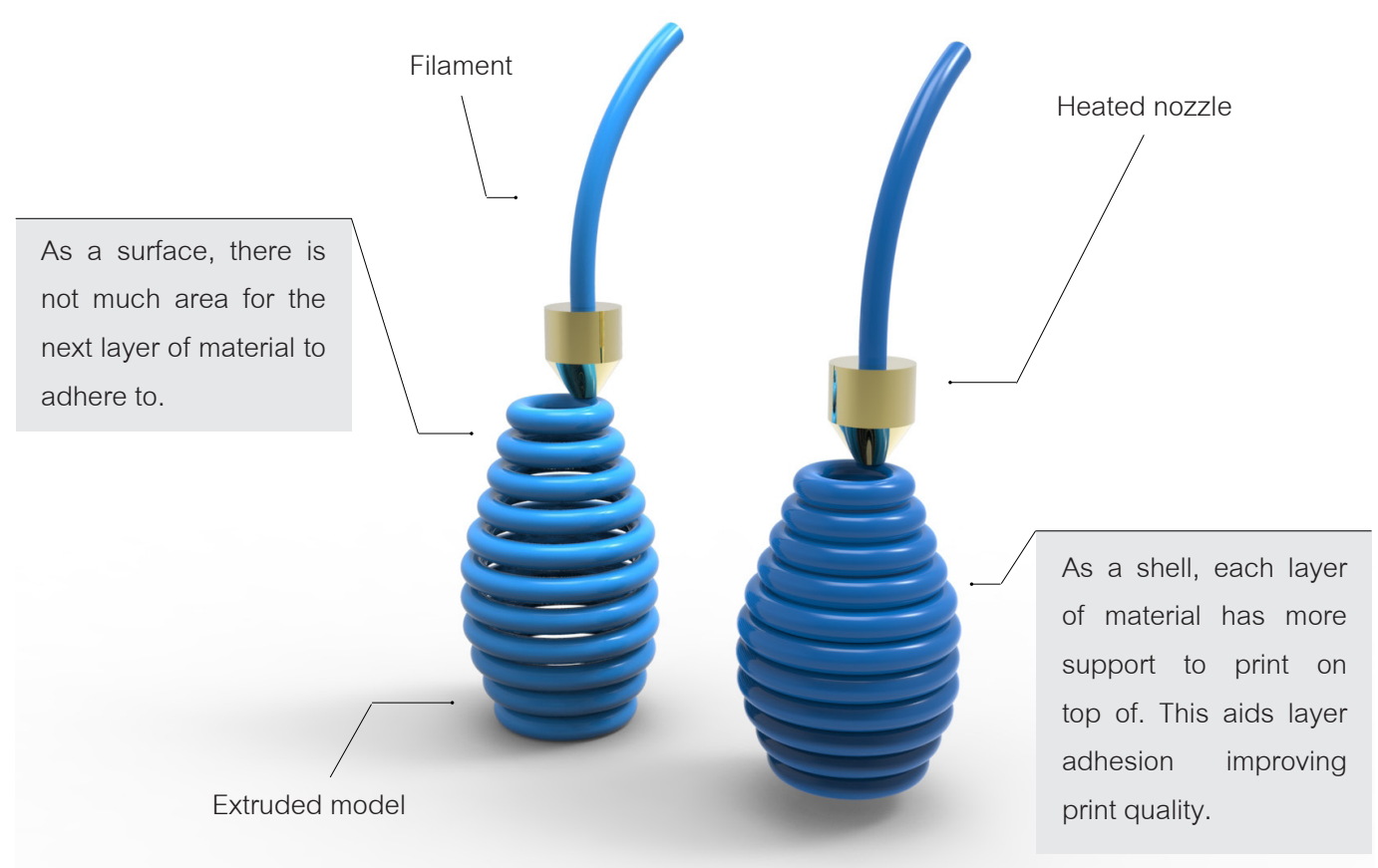

Figure 18 - Comparing how each wall thickness prints. 


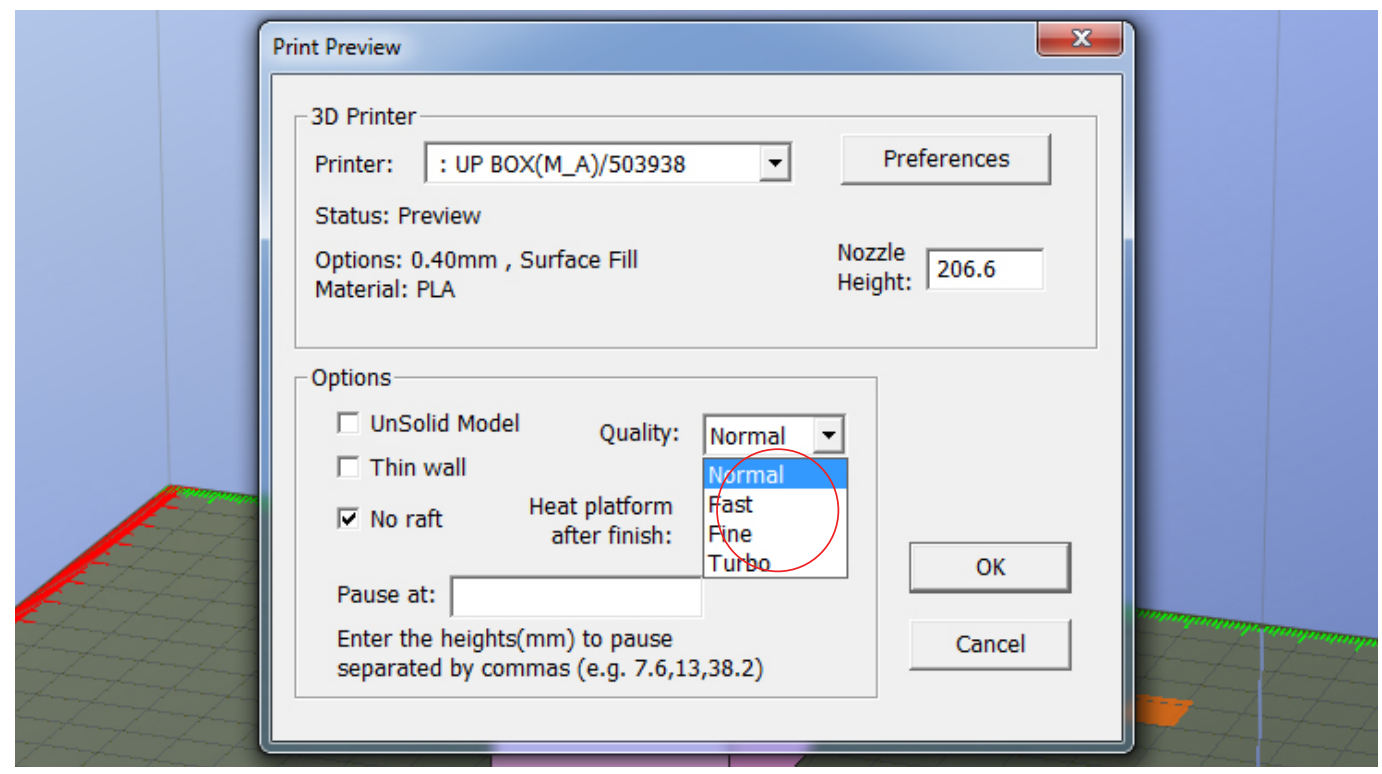

Printer speed is dictated as normal, fast, fine, and turbo
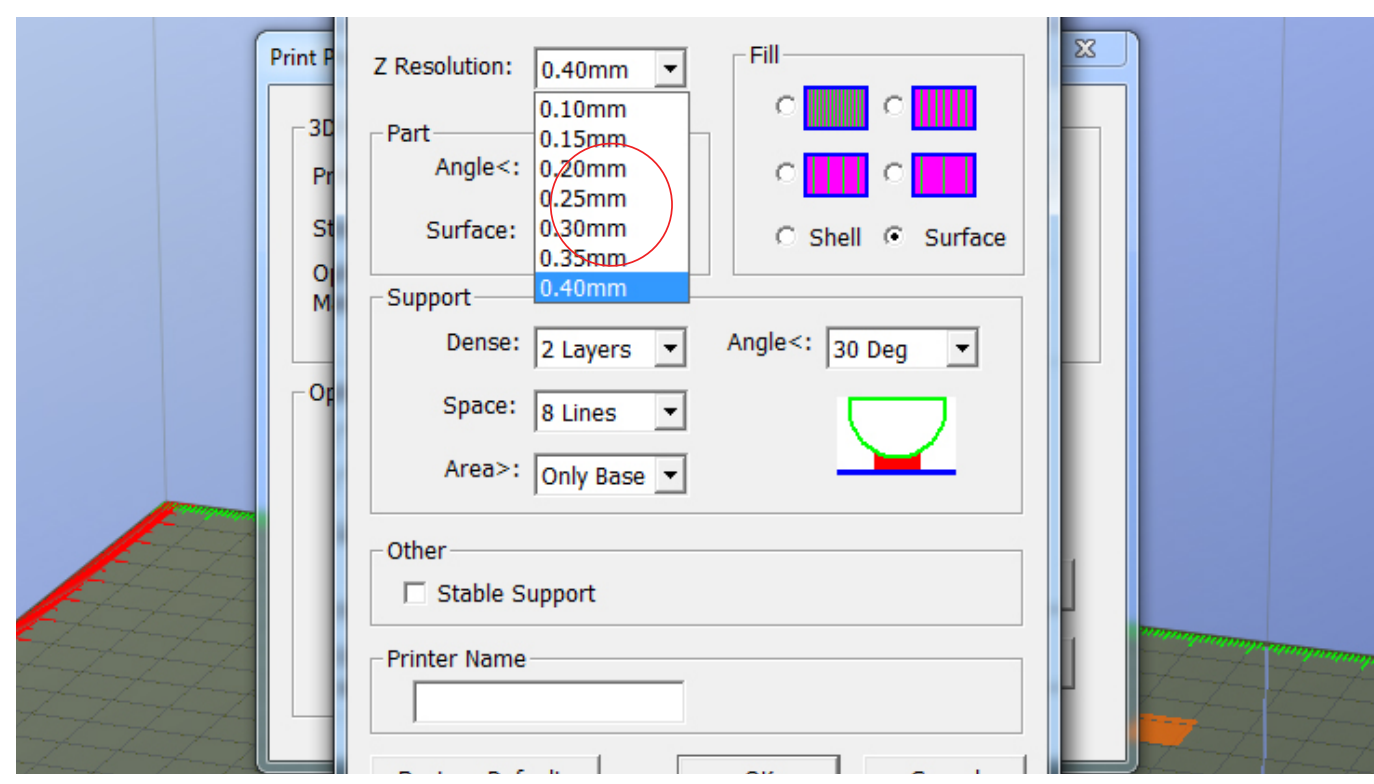
Layer heights can be set to $0.40,0.35,0.30,0.25,0.20,0.15,0.10 \mathrm{~mm}$.
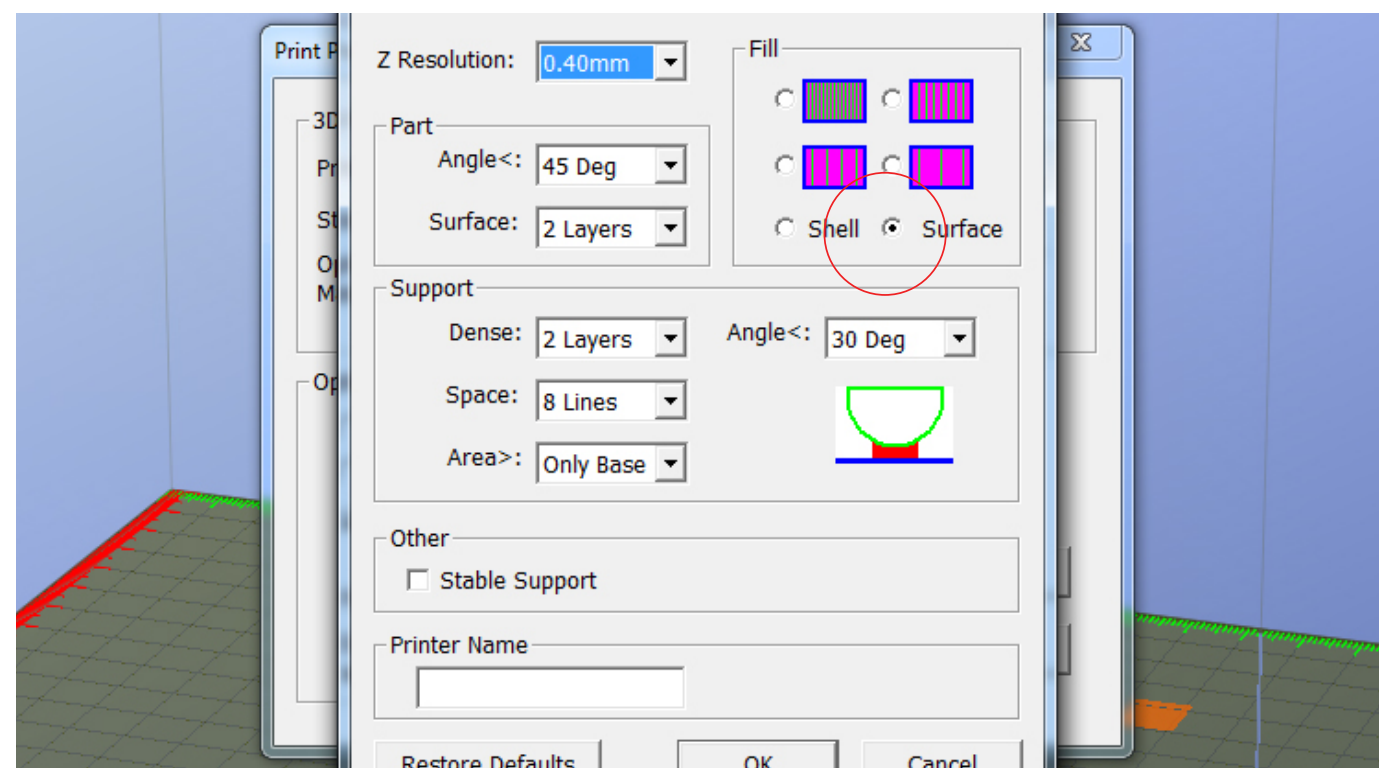

The wall layering can be set to shell (double layered), or a surface (single layered). 


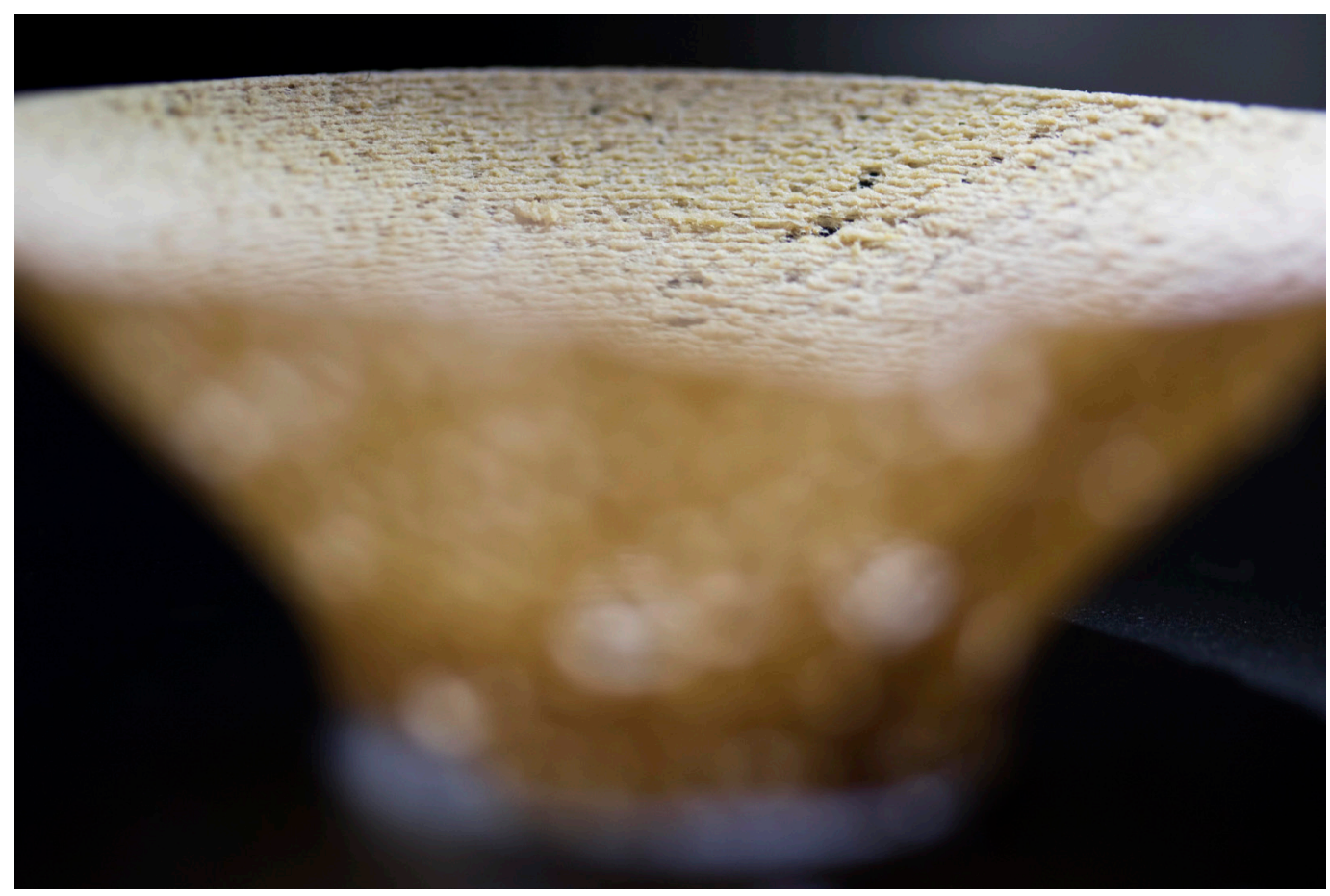

When printing the PLA wood material, natural textures are created.

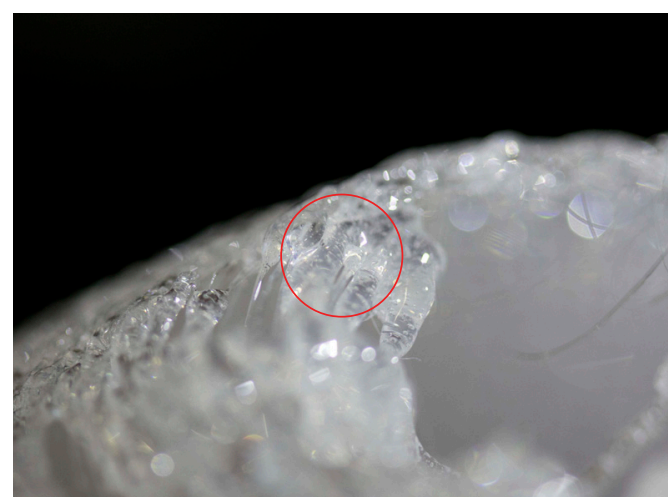

When the TPU is printed as a single wall layer it is more difficult to adhere the new layer of plastic to the previous layer. When this adhesion fails the material becomes uneven and can block the nozzle.

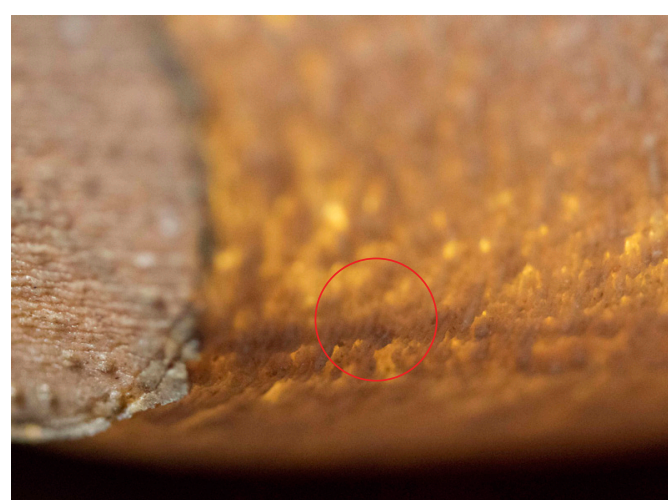

Interesting patterns are created by the 3D printer which are revealed when light is shone through the

print. 
The first mono-material models provided a basic understanding towards what forms and shapes FDM printing favours. The next stage explored using two different materials in a single print. These dual-material prints prompted a further investigation into speeds, temperatures and layer heights.

Exploring multi-materiality by printing one material inside another increased the strength of the model. The wooden PLA material is hard but brittle, snapping easily. The flexible TPU material is very strong and durable making it hard to break. How would the material quality of the printed object be affected if the hard, strong material was printed inside the durable, flexible material?

The Up Mini 3D printer used for these experiments has only a single extruder. This required the material filament changes to be made manually while the printer was mid-way through printing a model. As a result the dual-material changes would only happen within a horizontal axis of the model. This created a seat of experiments which are flat allowing the strength testing to come from the materials themselves, without the influence of the form.

When cold, the PLA plastic would not adhere to the flat layer of TPU (Figure 19). Having an object with a large flat surface causes each layer to cool down before the next molten layer is extruded on top. This made it difficult for the new layer of plastic to adhere to the previous. When the TPU plastic was heated, adhesion between materials was achievable.

The TPU material is strong when printed with a raft made of TPU flexible. Printing the raft in PLA and the base layer of the model with TPU, it removed easily and cleanly (Figure 20).

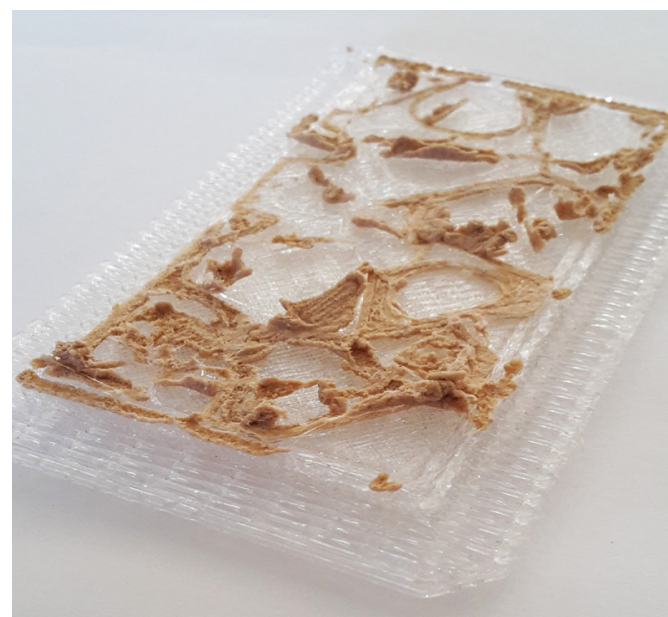

Figure 19

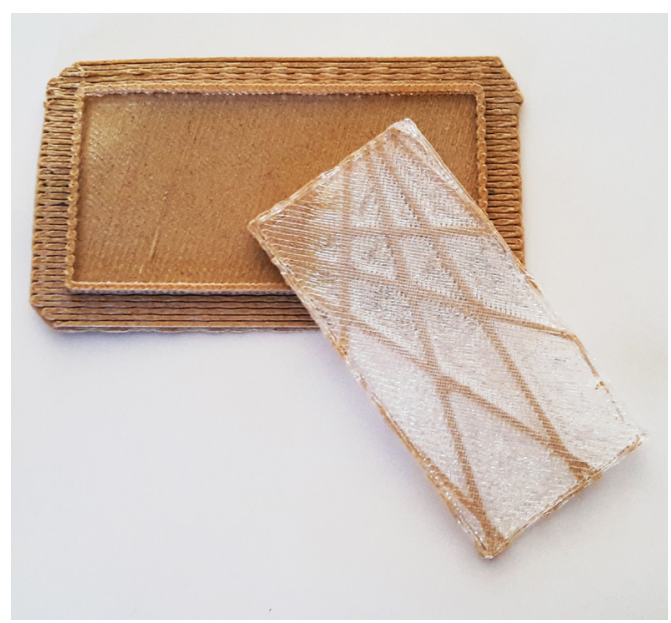

Figure 20 


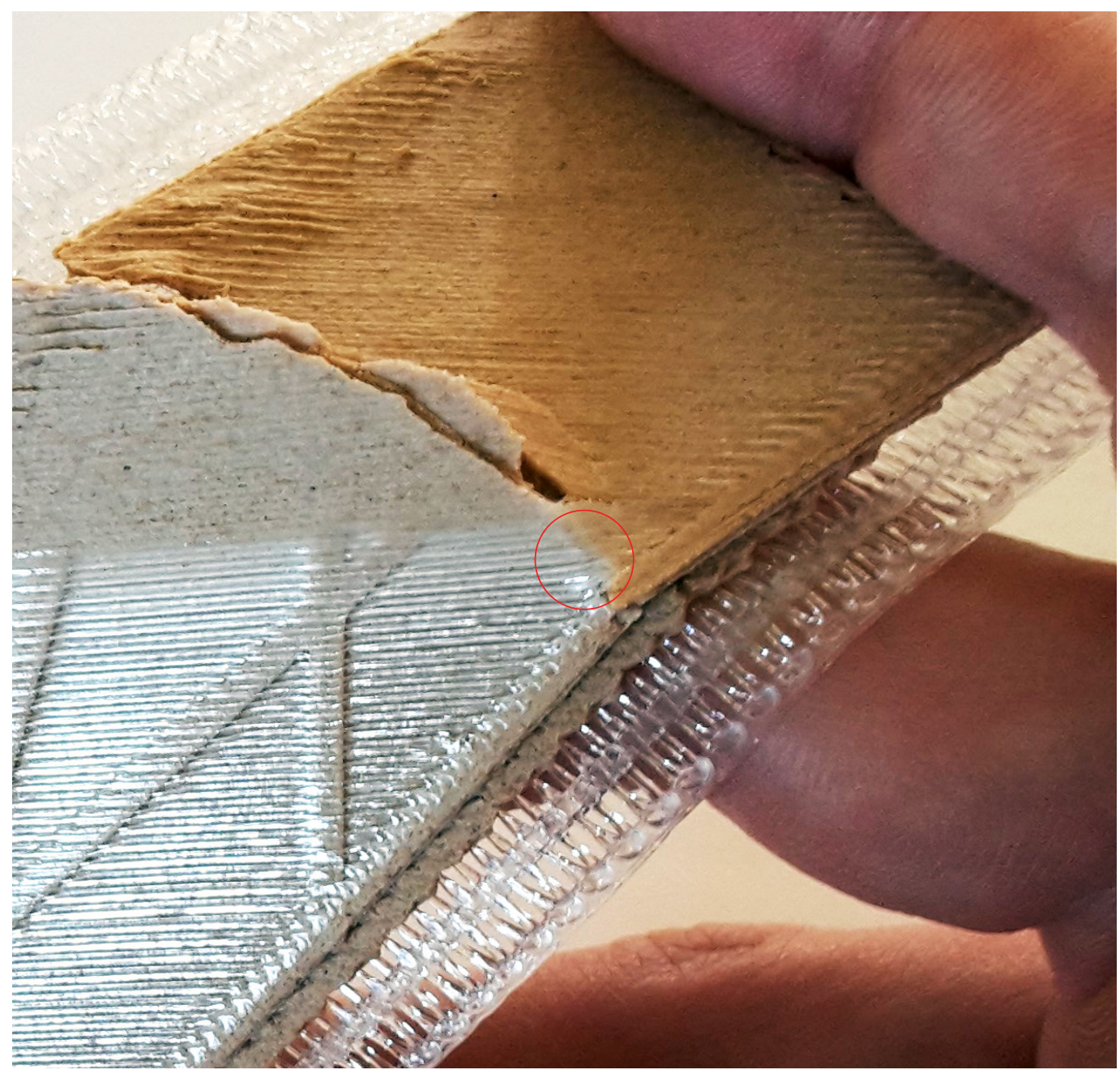

A clear example of how the TPU material aids strength and durability to a material which is hard and brittle.

\section{REFLECTION:}

Printing the PLA wood material inside the TPU plastic increased the strength and rigidity of the model. This test shows that there are benefits to designing an object to be 3D printed using two materials within a single print. This takes advantage of both material qualities creating a hybrid where efficiency and performance are increased. 


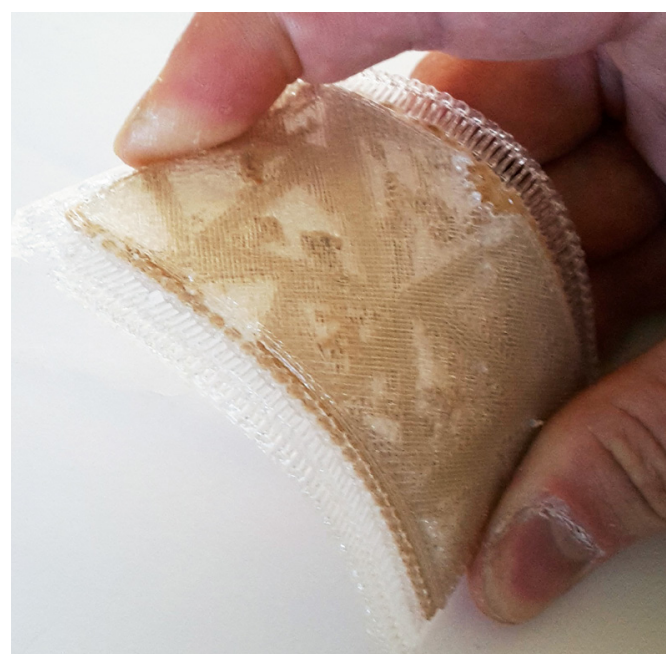

This model has a large area of PLA hard material creating a model that is rigid and durable.

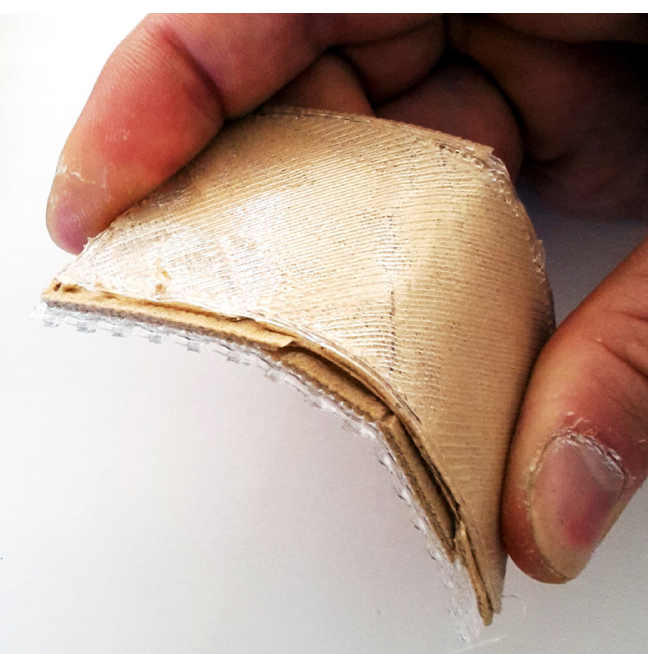

The TPU material holds together a print which would otherwise delaminate.

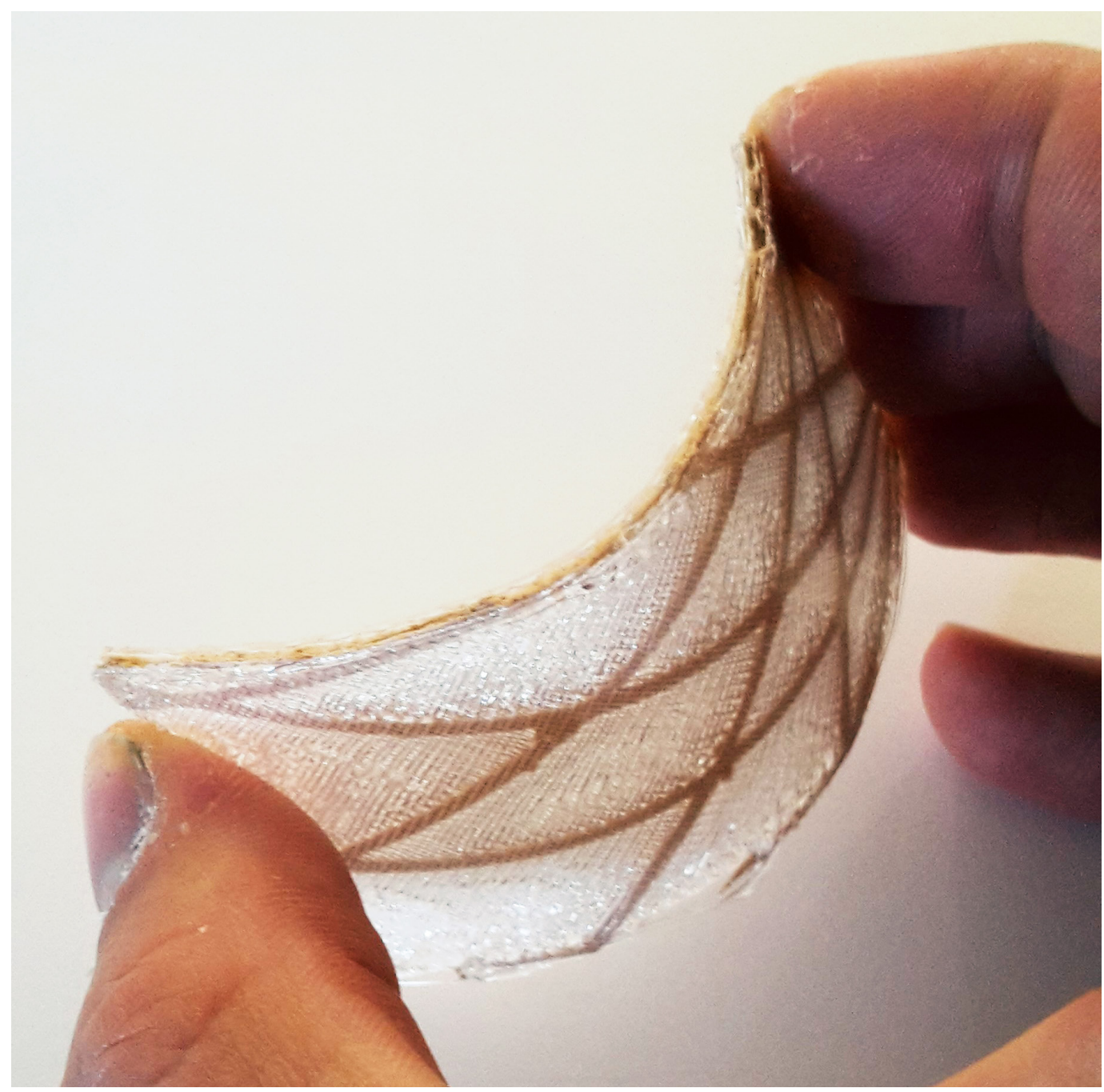

The effect the multi-material area creates when light is shone through the model, opens an opportunity to incorporate different levels of translucency and pattern. 
The set of initial Mono-Material and Dual-Material experiments derived a set of design rules to begin printing larger models using a combination of materials.

The rules encompassed:

\section{Material print speeds}

- To obtain successfully printed models, the $3 \mathrm{D}$ printer was set to the normal speed setting. This created a smooth bead of extruded plastic ensuring the model is of high quality.

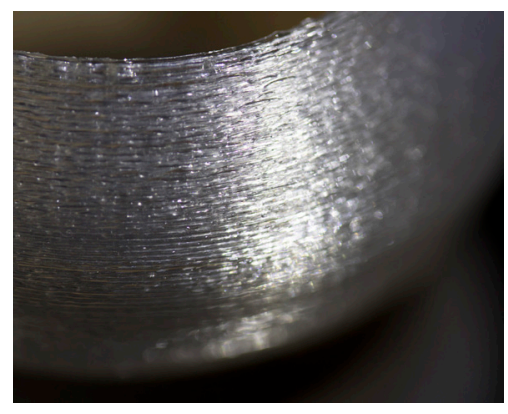

\section{Material strengths}

- Using two materials in a single print allows the model to take advantage of each individual material quality i.e. the strength of the TPU plastic, and the rigidity of PLA plastic.

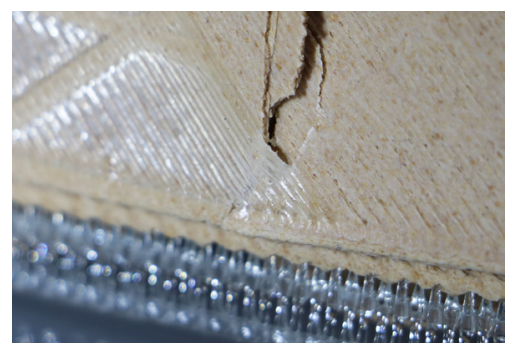

\section{Wall thicknesses}

- $\quad$ Selecting a single layered wall thickness allows the machine to bring unique qualities into the design of the model. Using a double layered wall thickness increased the quality of the printed object as well as allowing more exaggerated overhanging components.

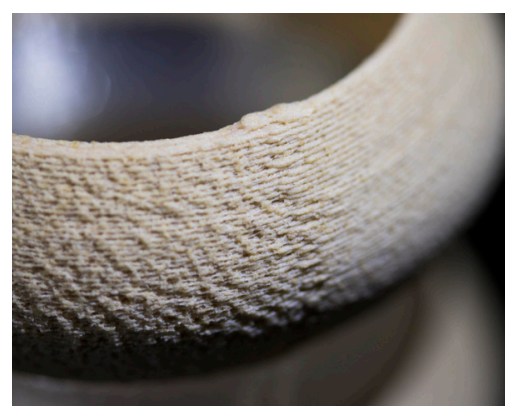

Print layer heights and extrusion flow rates.

- Having the print bed layer height set to $0.3 \mathrm{~mm}$ allows the nozzle to extrude a steady bead of material. This constant extrusion speed ensures a high quality, successful 3D printed model.

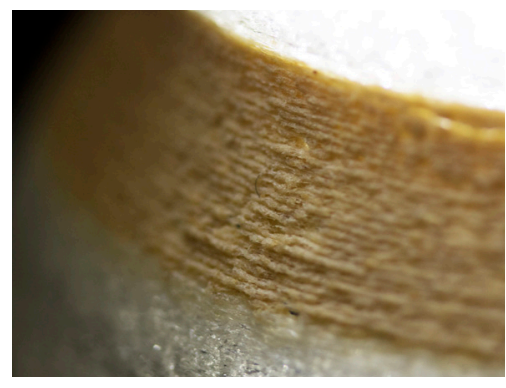

Initial exploration into build angles

- The initial investigation has illustrated the unique details created by overhanging draft angles.

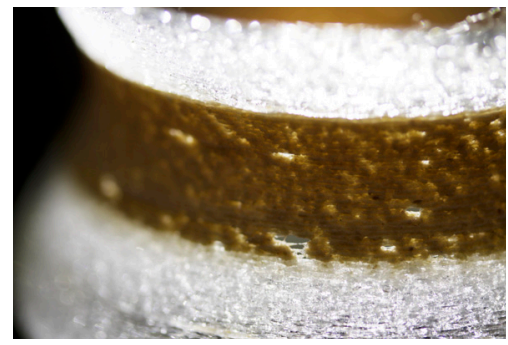




\section{TEMPERATURE REFLECTION:}

These initial small scale mono-material and dual-material 3D print tests are primarily focused on the printer settings with a disregard to the form of the objects being printed. This will be something explored further in the future. The PLA and TPU plastic's factory extrusion temperature recommendation is $210^{\circ} \mathrm{C}$. Exploring the ideal temperature setting for each of these materials will increase the print quality and strength of the materials. 


\section{TEMPERATURE INVESTIGATION}

To further build on the set of design rules, an investigation into customising the extrusion temperatures of the 3D printer called for a series of material tests in TPU flexible and PLA wood.

TPU flexible; the nozzle temperature was reduced to $190^{\circ} \mathrm{C}$, producing a high quality, clear print. The same models were then printed at $210^{\circ} \mathrm{C}, 230^{\circ} \mathrm{C}, 250^{\circ} \mathrm{C}$, and $270^{\circ} \mathrm{C}$. As the print temperature increased so did the density of the bubbles, reducing the translucency (Figure 21). The bubbles were produced due to the material boiling within the nozzle.

PLA wood; the temperature was reduced to $190^{\circ} \mathrm{C}$. Once again any lower than this, and the nozzle would block. The temperature was increased to $210^{\circ} \mathrm{C}, 230^{\circ} \mathrm{C}, 250^{\circ} \mathrm{C}, 270^{\circ} \mathrm{C}$, and $290^{\circ} \mathrm{C}$. As the temperature increased the printed material became darker as it burnt inside the nozzle of the printer (Figure $22 \& 23$ ). When the material was printing at $290^{\circ} \mathrm{C}$ the wood material became black and brittle. At $200^{\circ} \mathrm{C}$, it was stable allowing it to bridge gaps with the least amount of drooping.

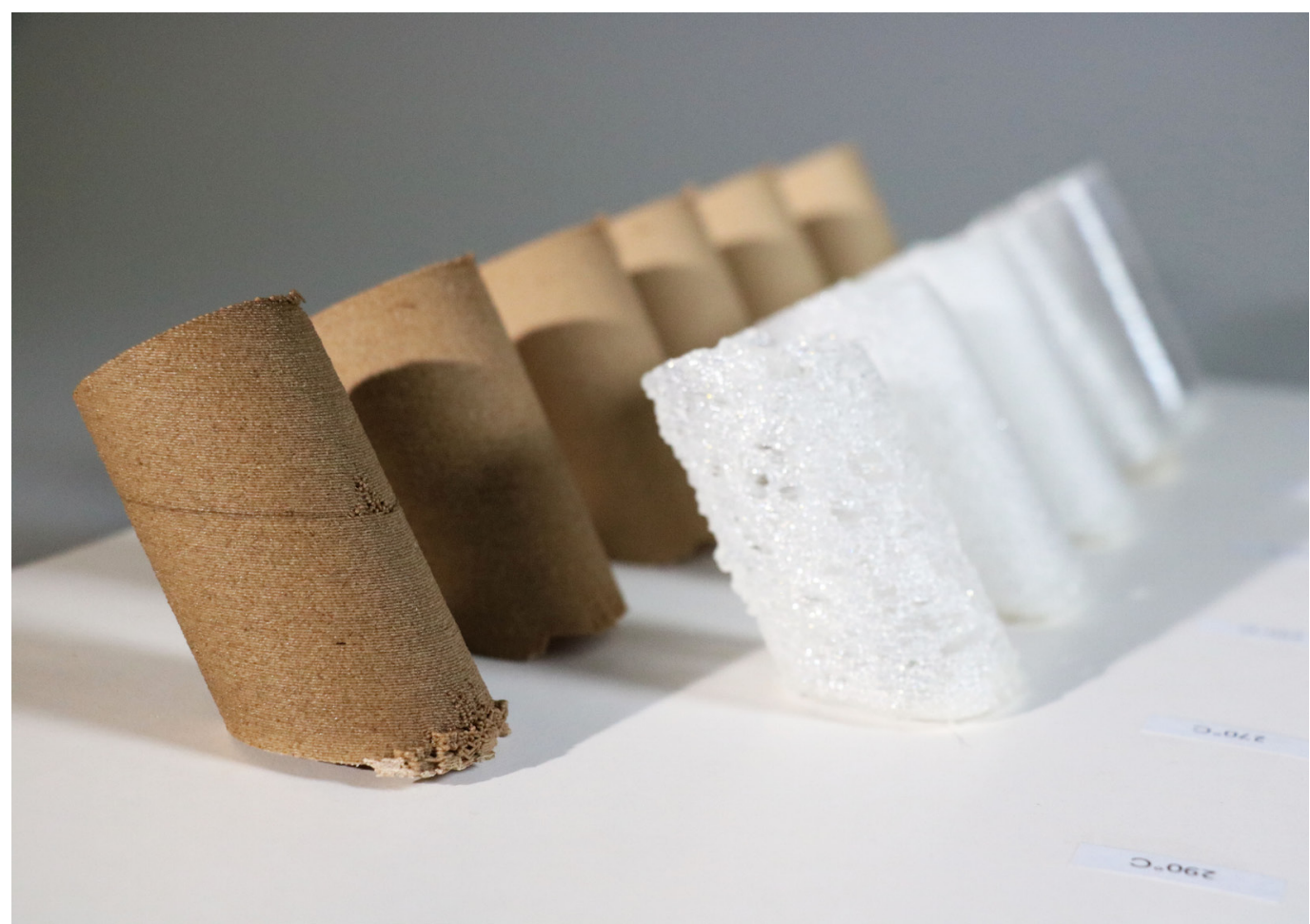

A series of PLA wood and TPU flexible print temperature tests ranging from $190^{\circ} \mathrm{C}$ to $290^{\circ} \mathrm{C}$. 


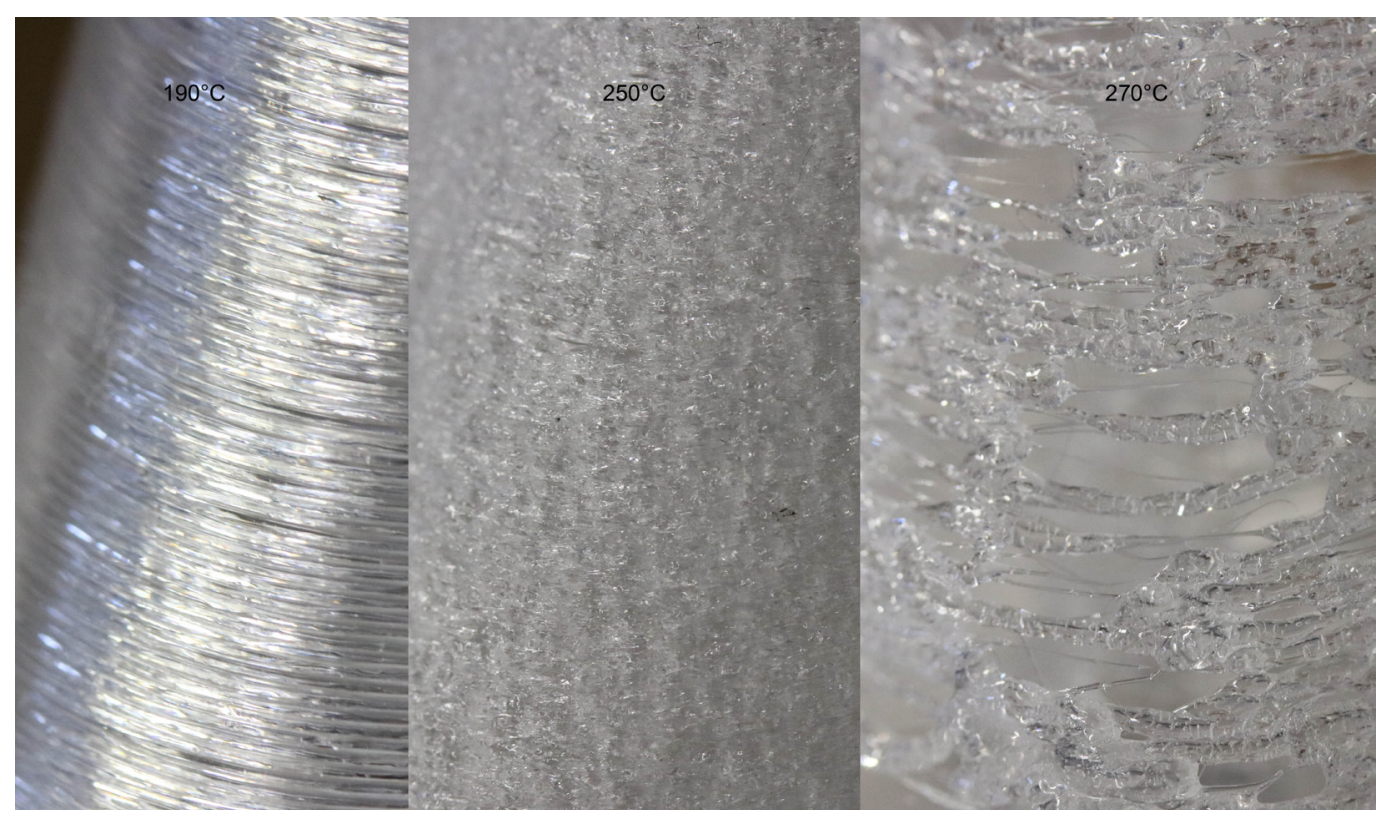

Figure 21 - Comparing TPU plastic printed at $190^{\circ} \mathrm{C}, 250^{\circ} \mathrm{C}, 270^{\circ} \mathrm{C}$

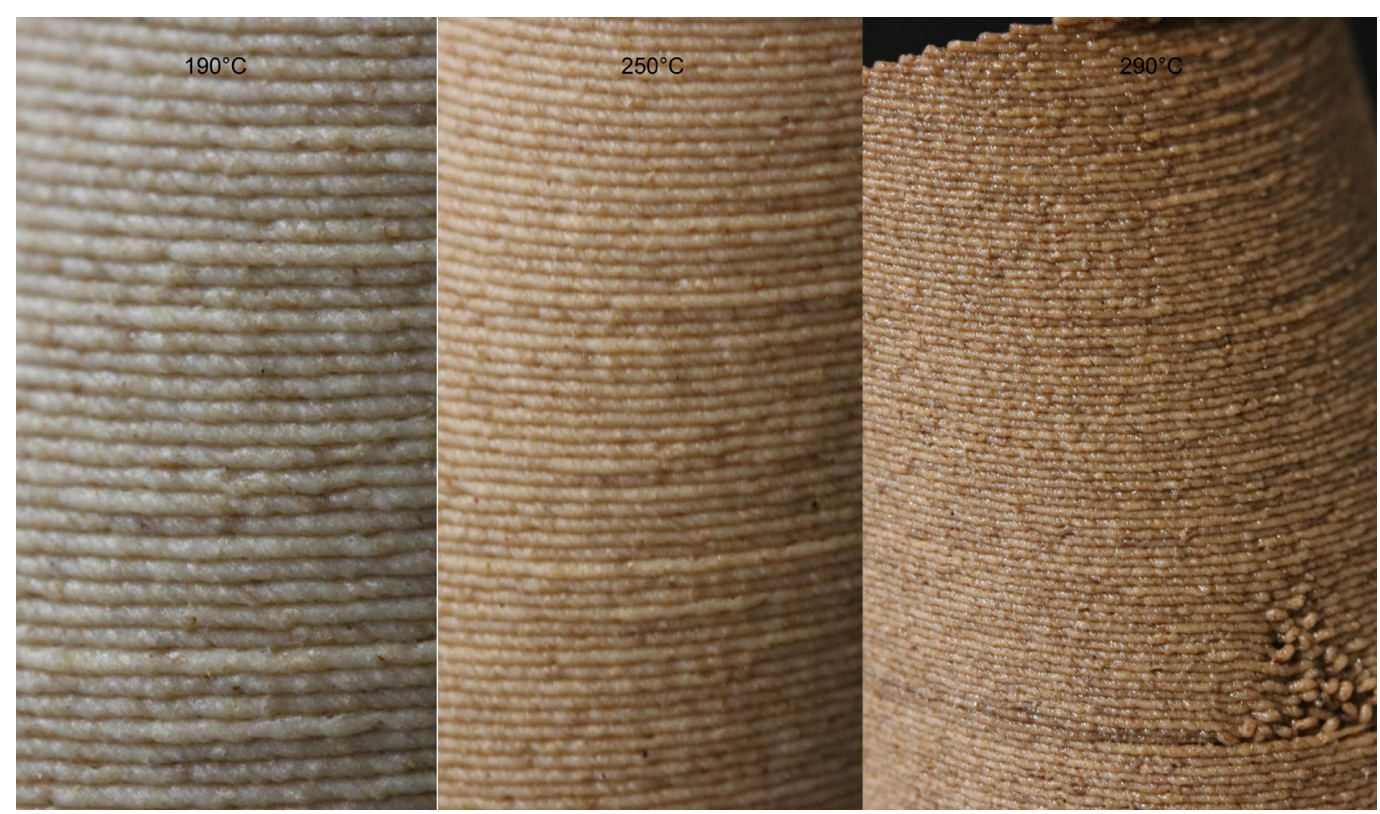

Figure 22 - Comparing PLA wood plastic printed at $190^{\circ} \mathrm{C}, 250^{\circ} \mathrm{C}, 270^{\circ} \mathrm{C}$.

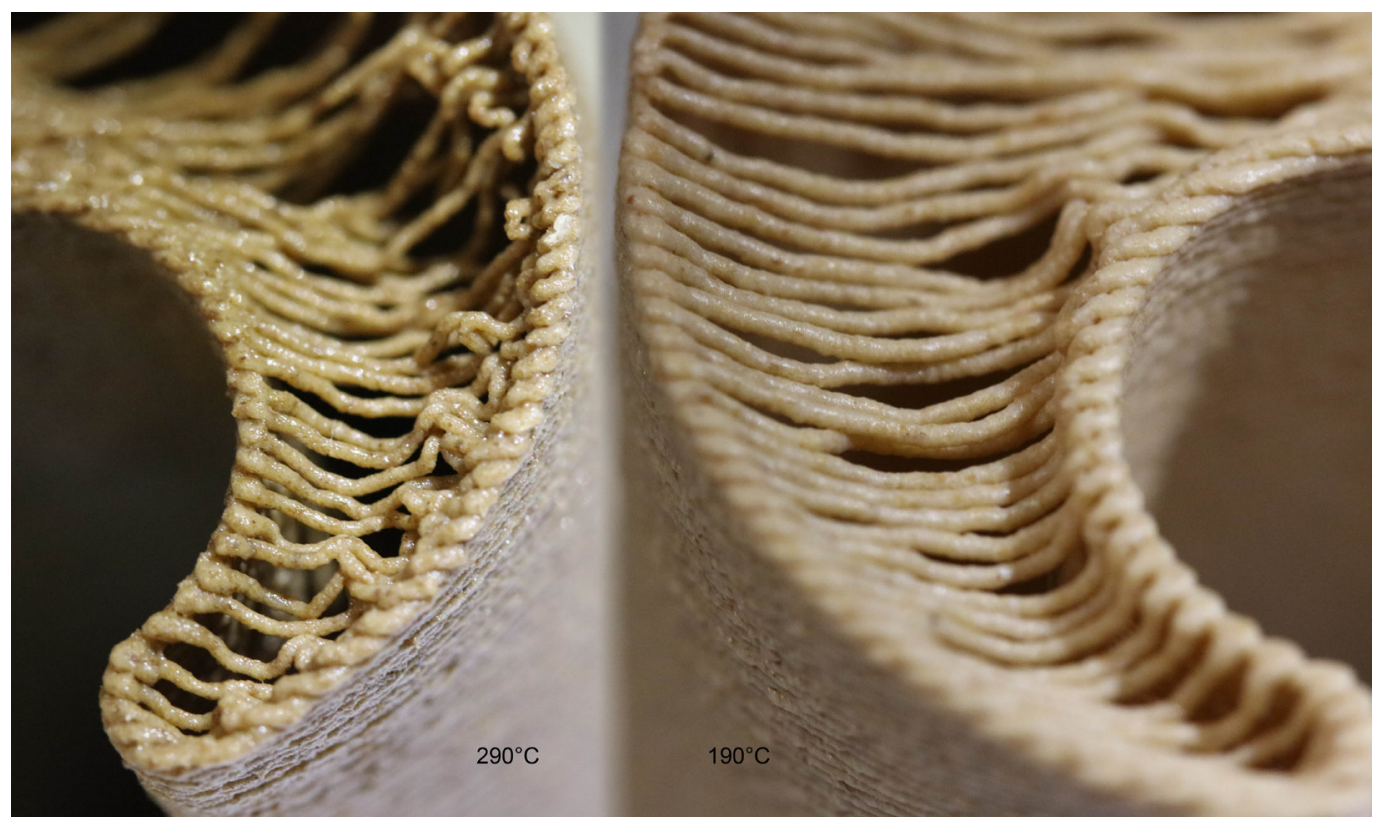

Figure 23 - Comparing the bridging capabilities of PLA wood plastic printed at $190^{\circ} \mathrm{C}$ and $290^{\circ} \mathrm{C}$. 
TEMPERATURE REFLECTION:

This temperature experimentation has established the ideal extrusion temperature needed to print both PLA wood and TPU flexible. Printing at $200^{\circ} \mathrm{C}$ improves strength and creates a more desirable aesthetic. The lower the temperature, the lower viscosity it is when extruded from the nozzle. This means that the adhesion between layers at a lower temperature is weaker than that of a higher temperature. The balance between adhesion and material quality that ensures a successful print.
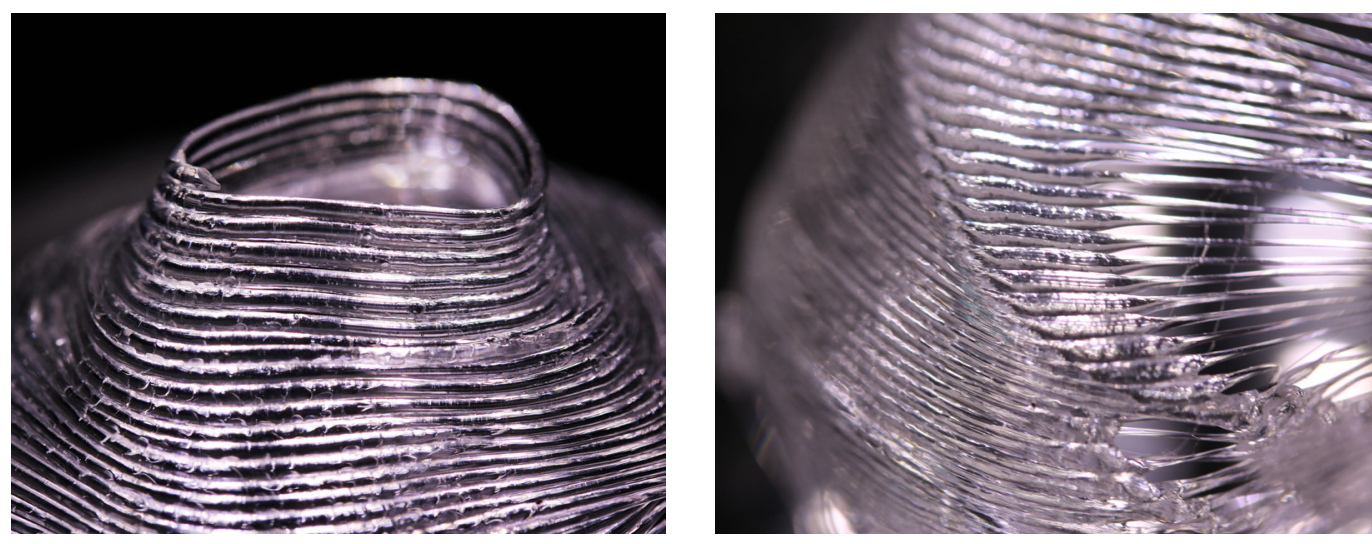

Being printed at $200^{\circ} \mathrm{C}$ you can see this TPU flexible material is extremely clear. This means it is high quality and strong

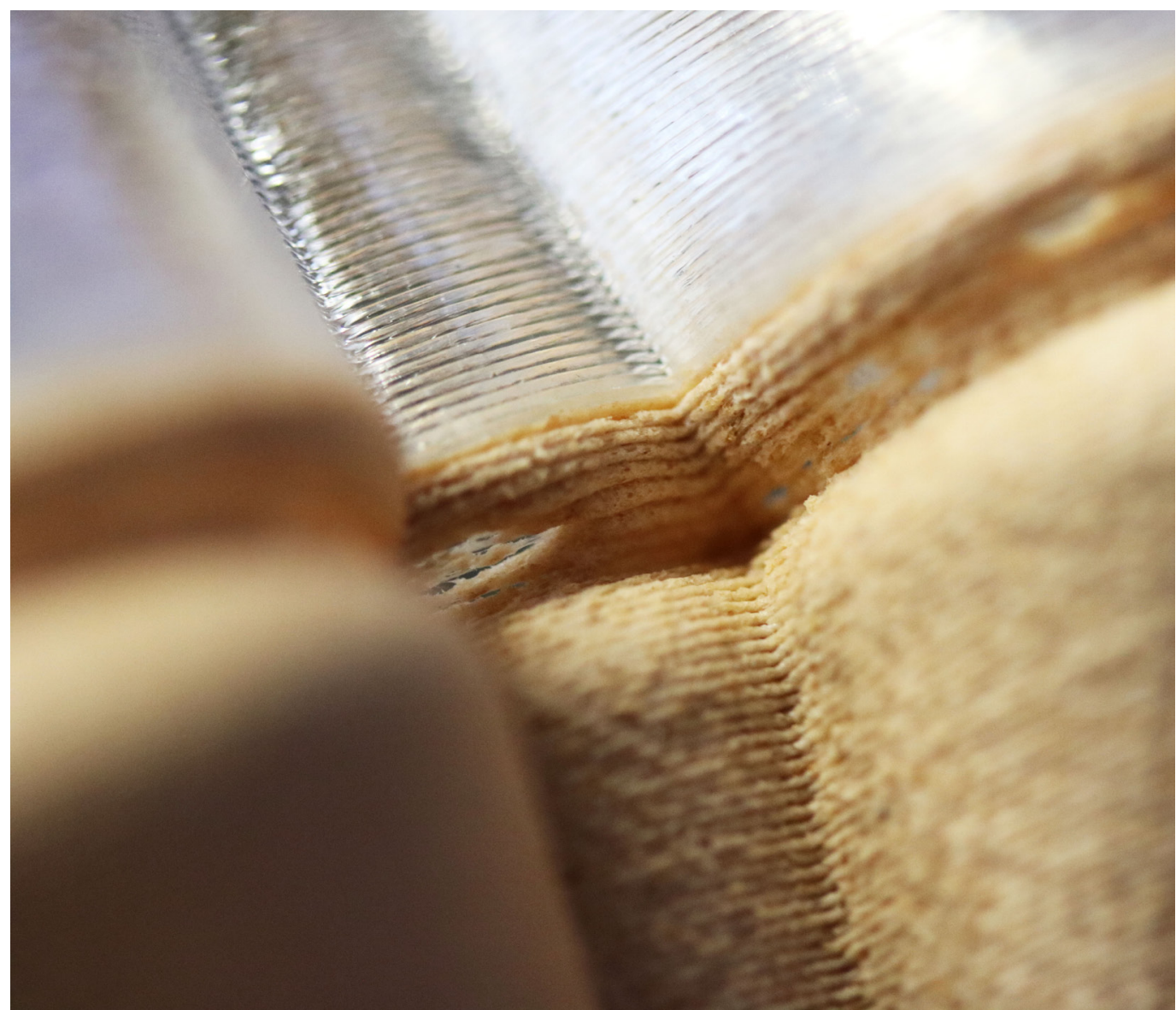

Printing both materials at $200^{\circ} \mathrm{C}$ creates a high quality multi-material 3D printed object. 

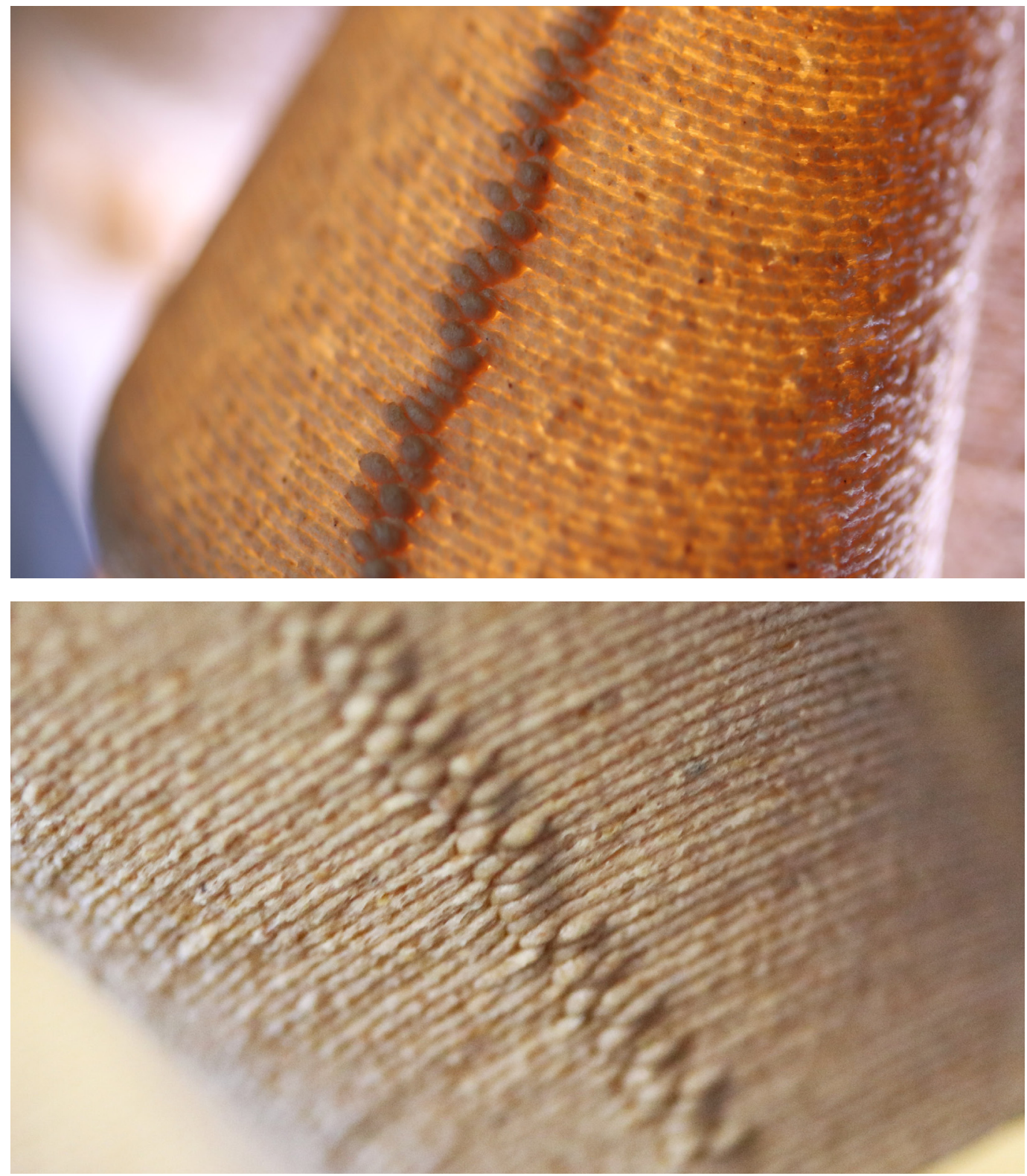

When 3D printing an object the start and end of each layer creates a seam up the side of the object. This seam has been highlighted as it is a part of the 3D printing process that should not be hidden but exaggerated.

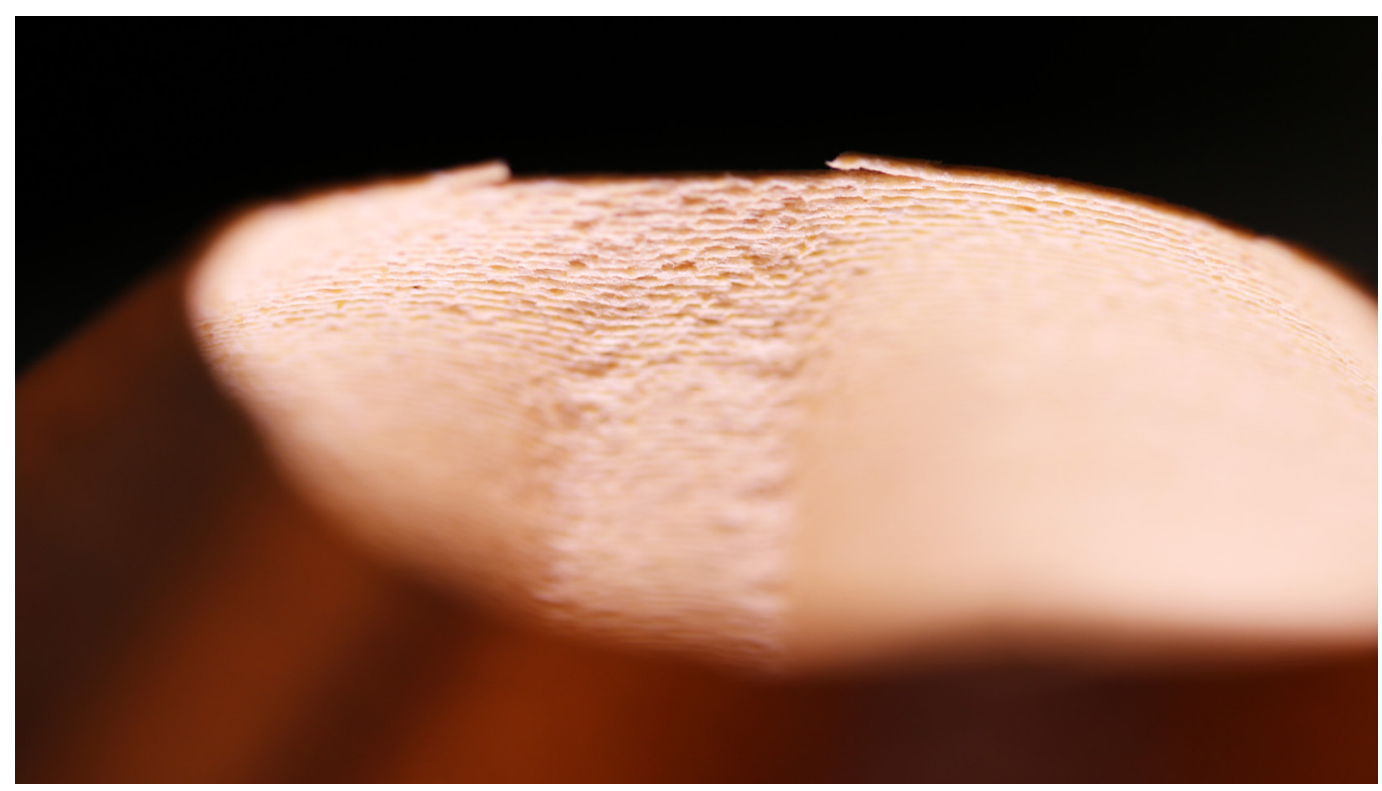

The layer lines have created textures when being printed at this lower temperature. 


\section{DRAFT ANGLE INVESTIGATION}

These multi-material combinations of PLA and TPU explore the limitations of printing with an extreme overhanging angle without using any support. Initial material tests revealed that printing overhanging draft angles created unique aesthetic opportunities.

Figure 24 is printed using PLA white plastic. This material behaves differently to the PLA wood having a lower viscosity when extruded, creating interesting drooping patterns. The draft angle influences how large or small this pattern is. The larger the draft angle, the less surface area the new bead of extruded plastic has to adhere to, creating a larger pattern.
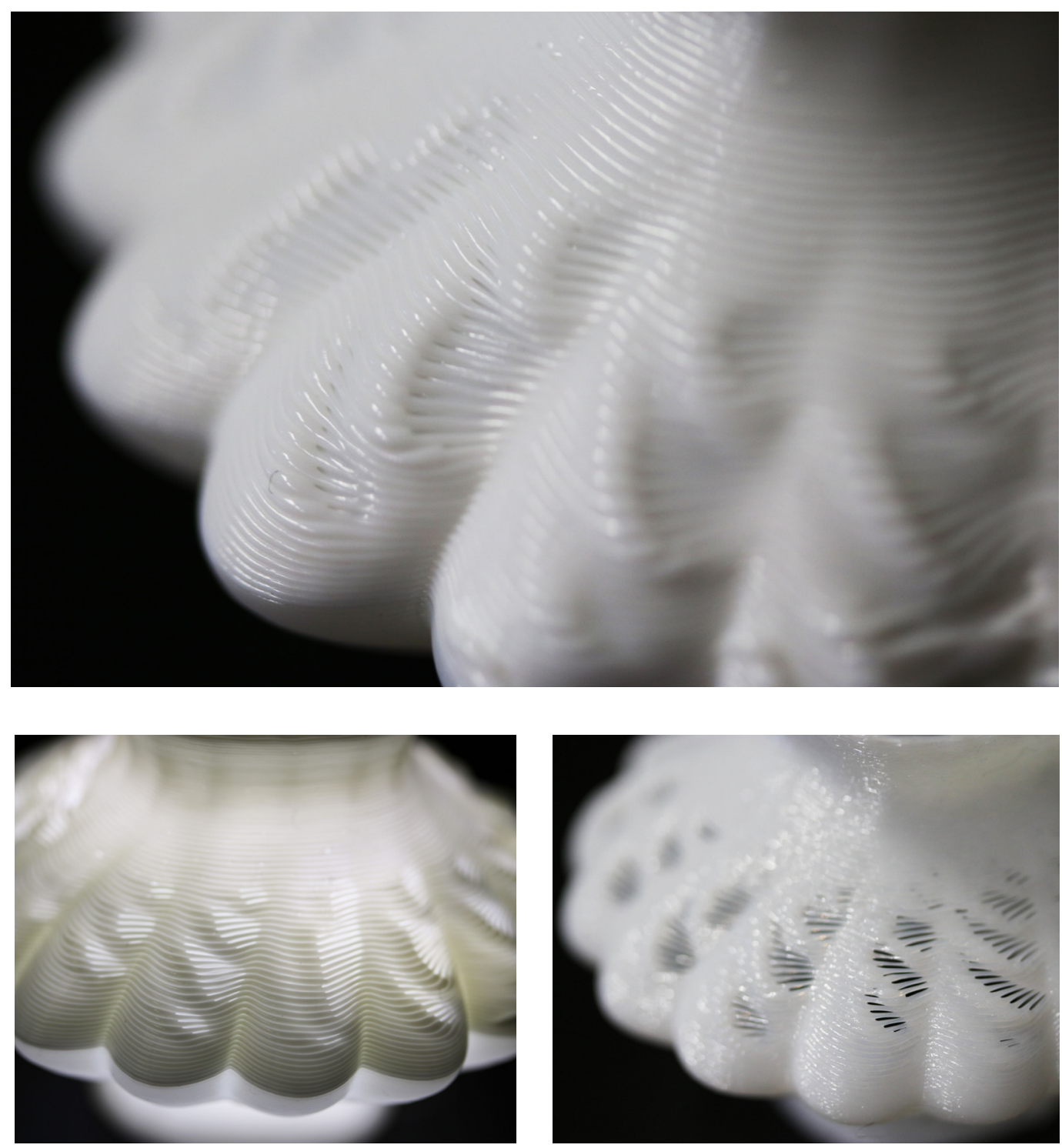

Due to the shape of the model a pattern has been created. This complements the design of the object adding to its aesthetic value. 


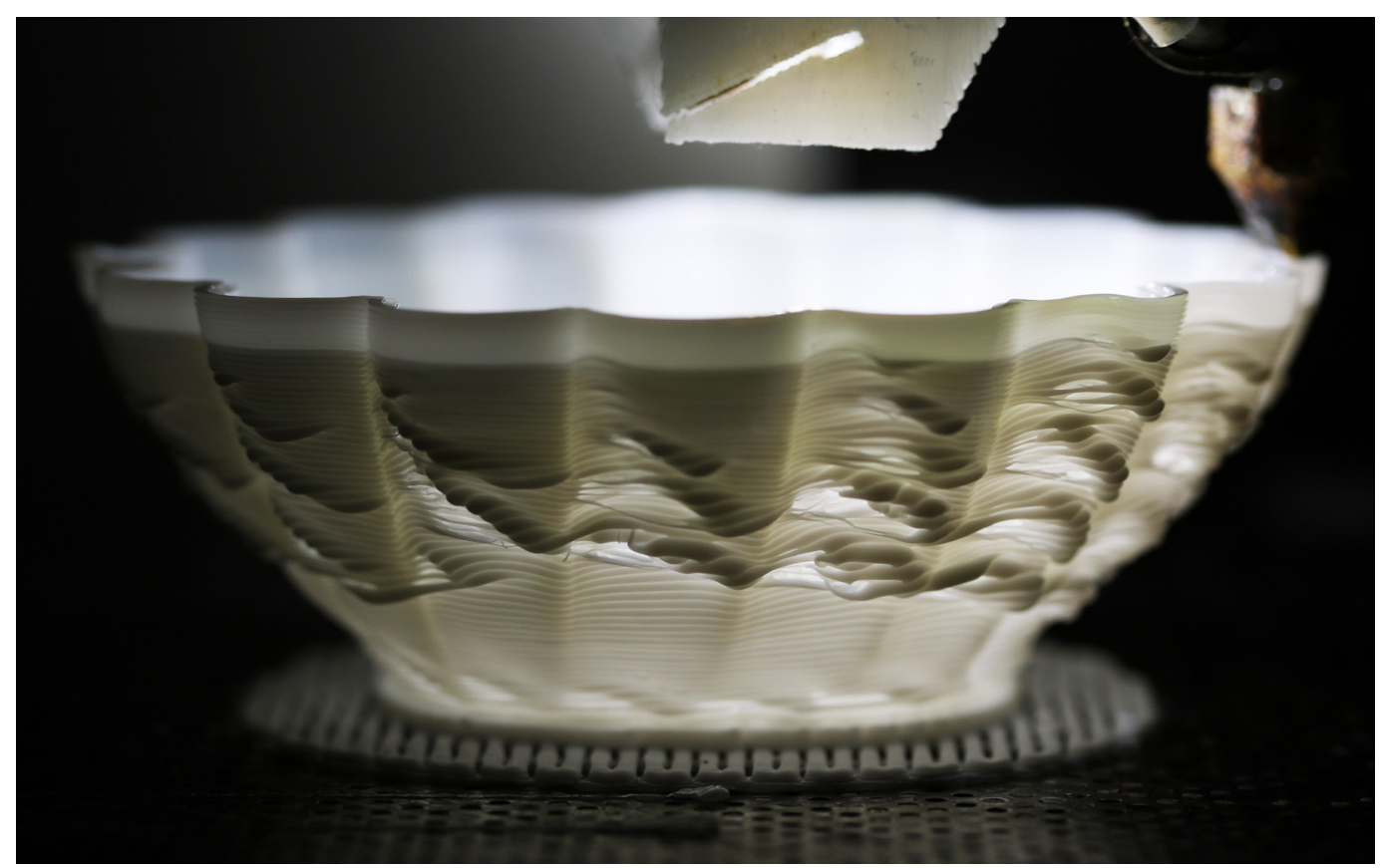

Figure 24 - First print using PLA White plastic.

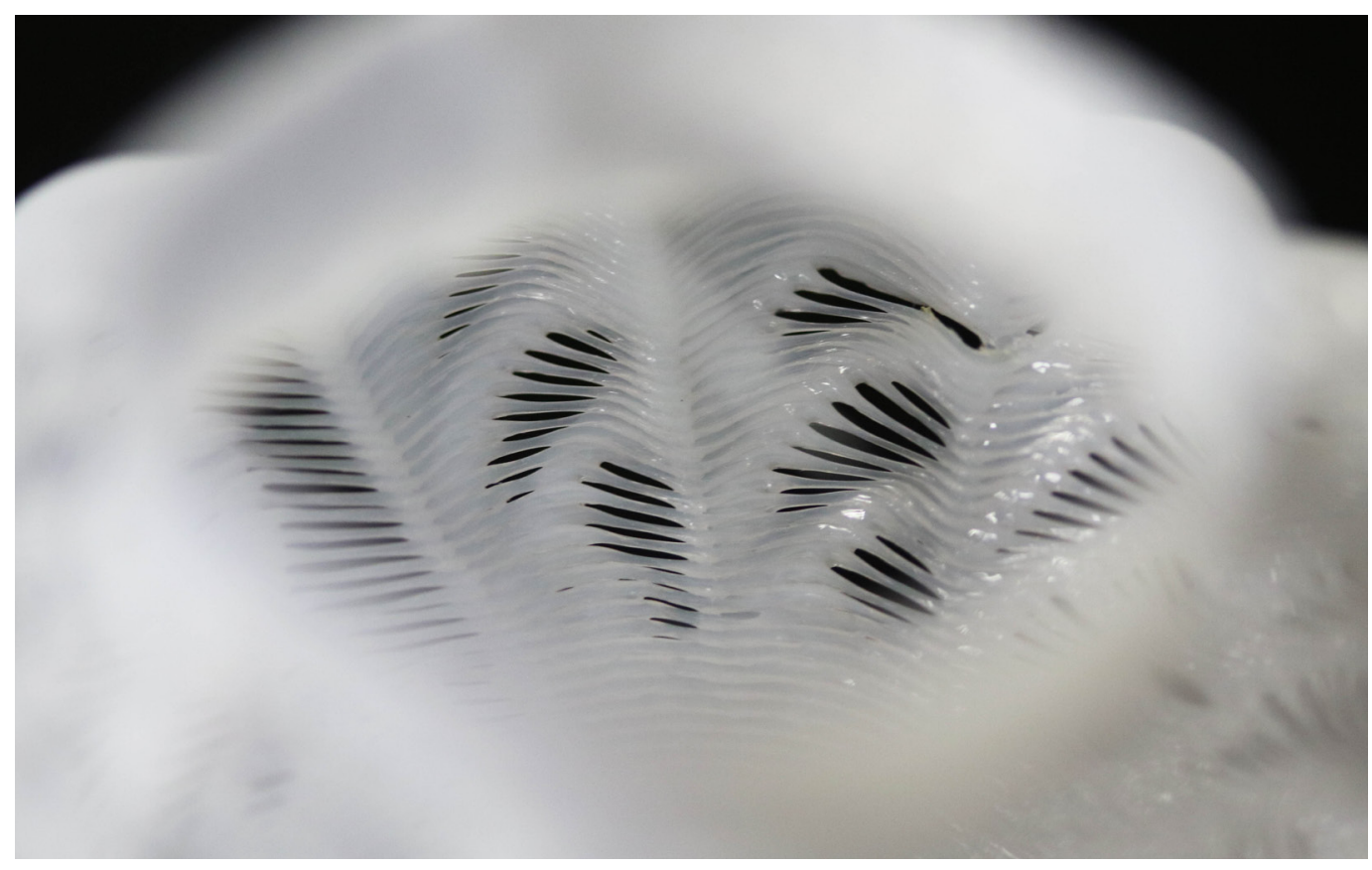

Patterning created by an overhanging angle. 


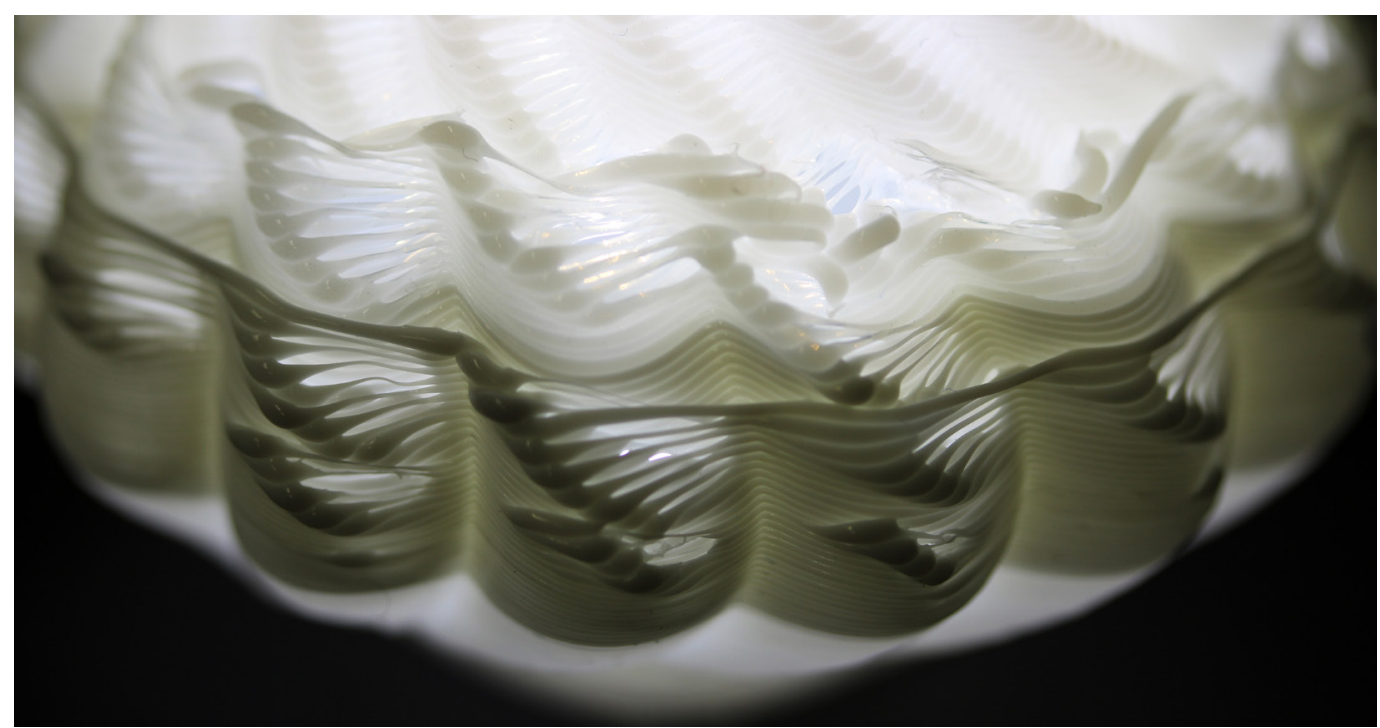

This image illustrates how light moves through the different materials. On the top is TPU white, and on the bottom is PLA white.

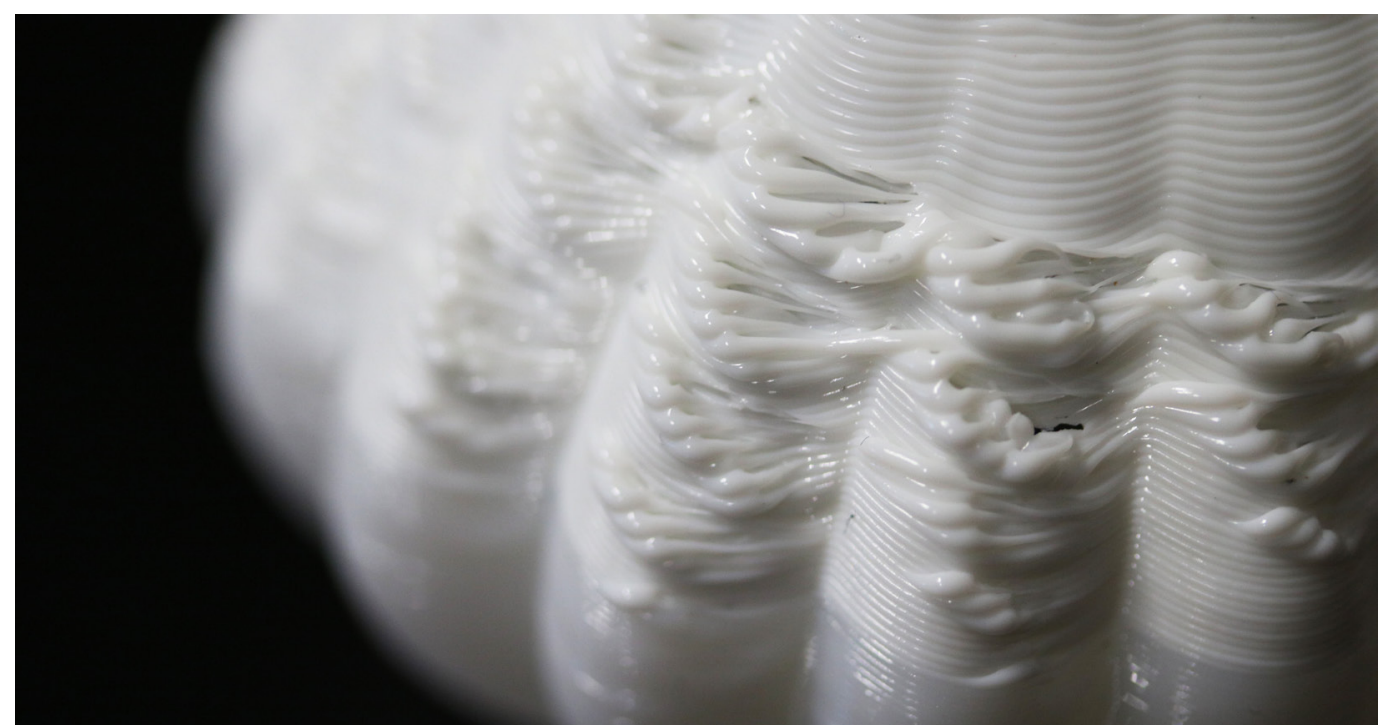

The overhang angle has caused a failure in the printed object. If this was just a single occurrence it may have ruined the model, but as it is recurring it has created a pattern that reinforces the design of the object.

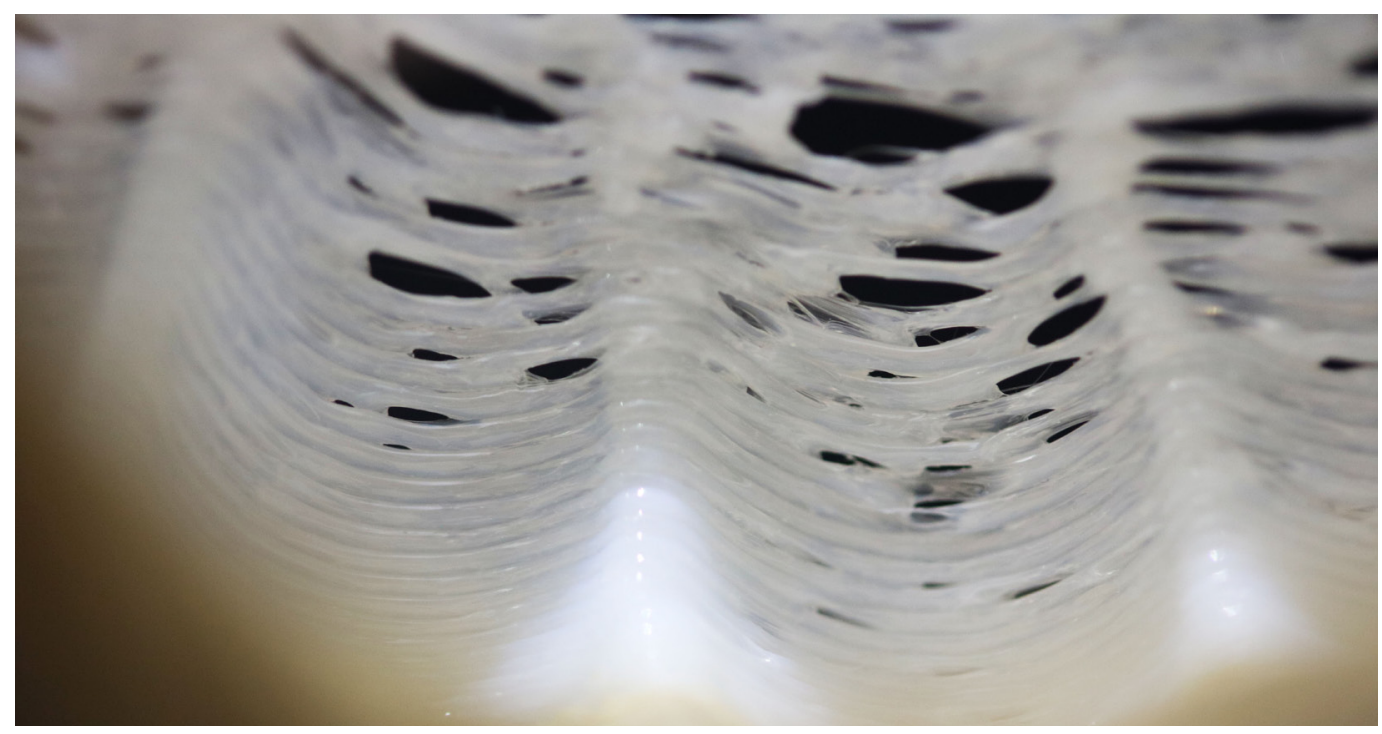

Patterning created in the TPU material as the angle is increased. 


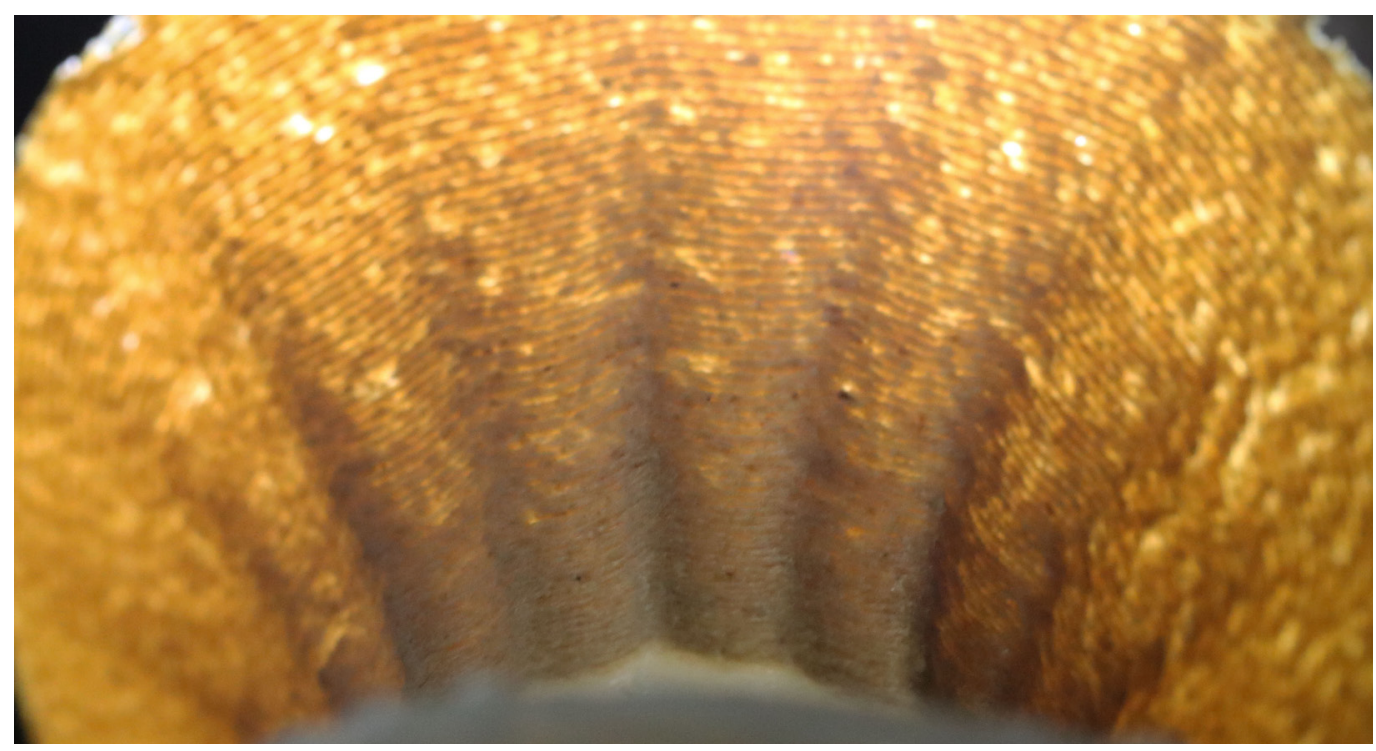

The change in transparency illustrates how the overhanging layers have less material bonding each bead of material together.

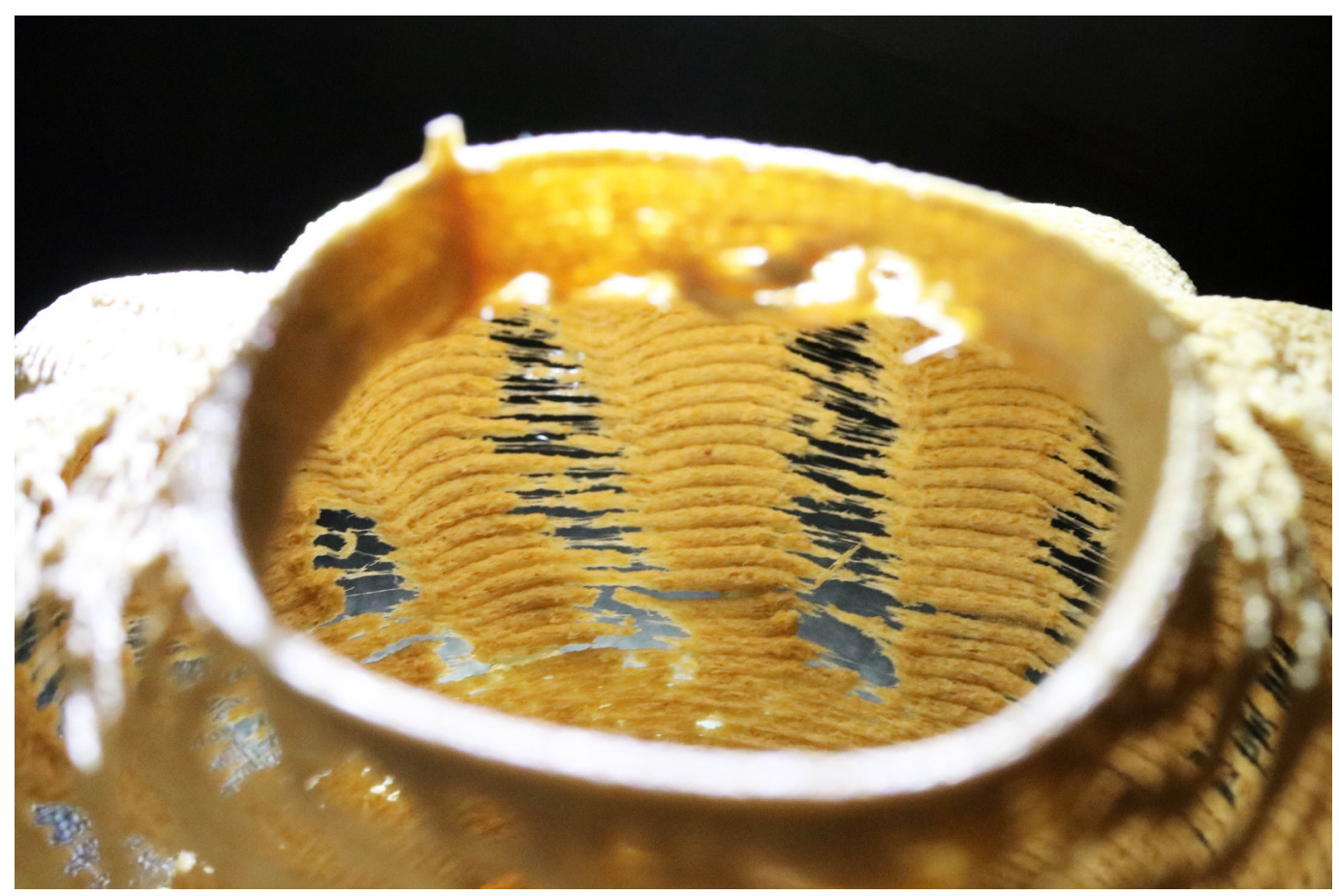

The increased angle of the object has forced the PLA wood filament to stretch and span across a gap.
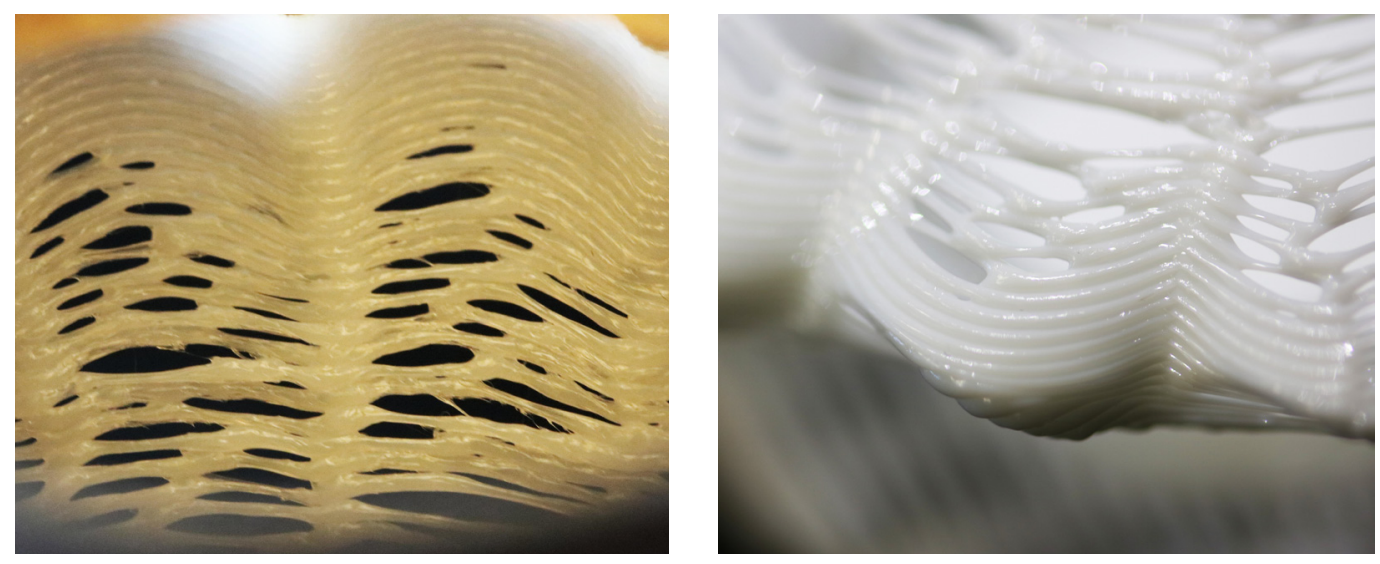

The draft angle in these two prints has caused the material to create a pattern similar to some of the other printed objects. 
The aim of these experiments is to evaluate how the material reacts when identical objects are printed at increasing draft angles. Beginning at 30 degrees and rotating all the way to 85 degrees in 5 degree increments (Figure 25).

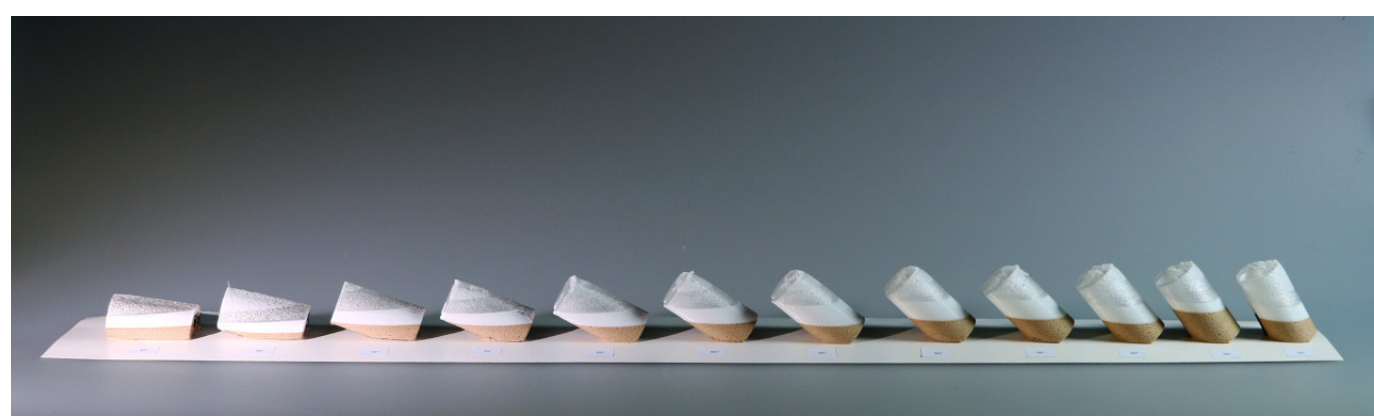

Figure 25 - 3D printed draft angle experiment.

\section{DRAFT ANGLE REFLECTION:}

These experiments revealed two factors.

- $\quad$ Firstly, when printing an object with a certain degree of overhang, (without any support material), the printer struggled to adhere one layer to another, creating interesting patterns on the surface of the object. As this draft angle was increased the patterns became larger and more defined. These patterns were more apparent when printed with a single layered surface, rather than a double layered shell. These patterns could be seen as flaws in the printing, but I see them as an opportunity to bring the 3D printers machine craft into the design of the printed object.

- Secondly, each material acts in a unique way when overhanging. Figure 26 shows how the wooden PLA plastic can span short distances producing different qualities to the other materials. The PLA white material spans across gaps creating a clean, glossy pattern (Figure 27). The TPU clear and TPU white also span across gaps, but with smaller shapes, it can sometimes stop extruding cleanly and end up creating build-ups of molten plastic. (Figure 28).

From these angle tests, it is clear that when printing overhanging angles each material creates individual patterns and qualities that are difficult to replicate. 


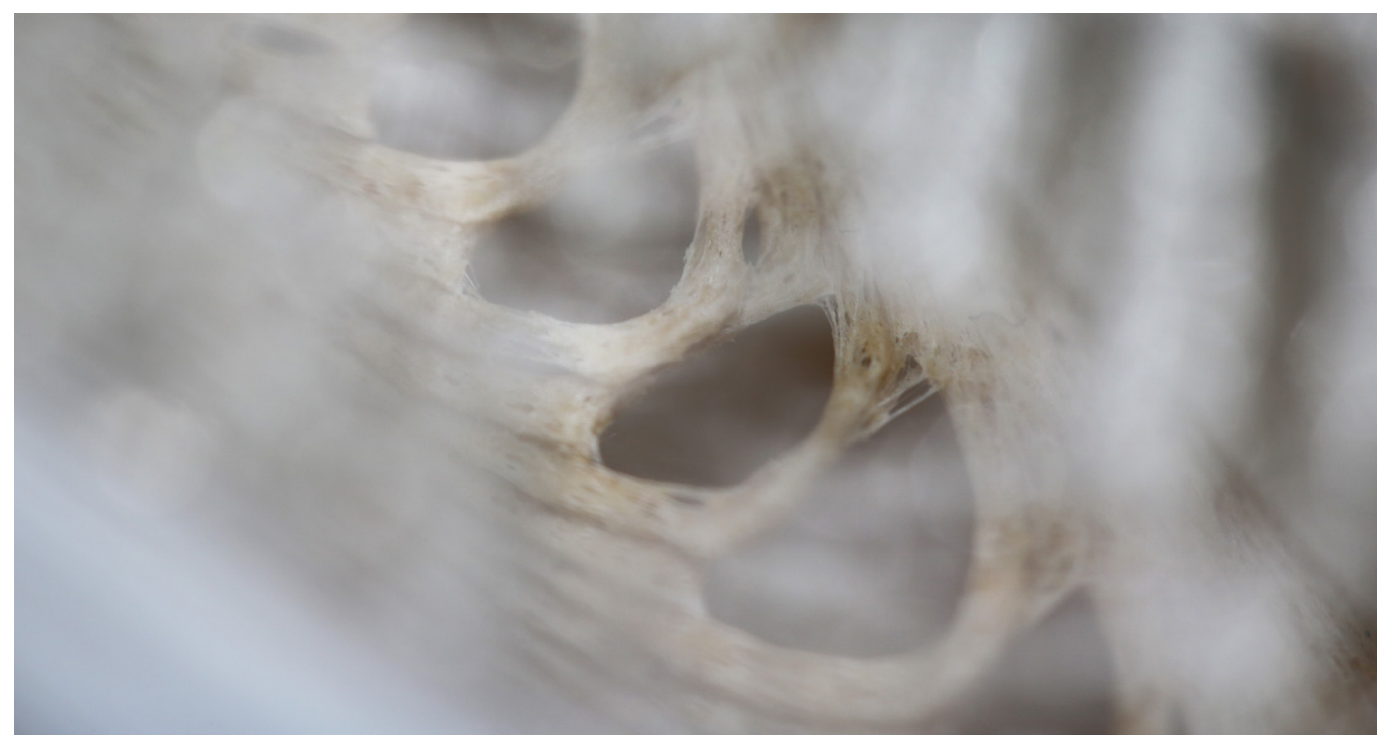

Figure 26 - PLA wood spanning a short distance.

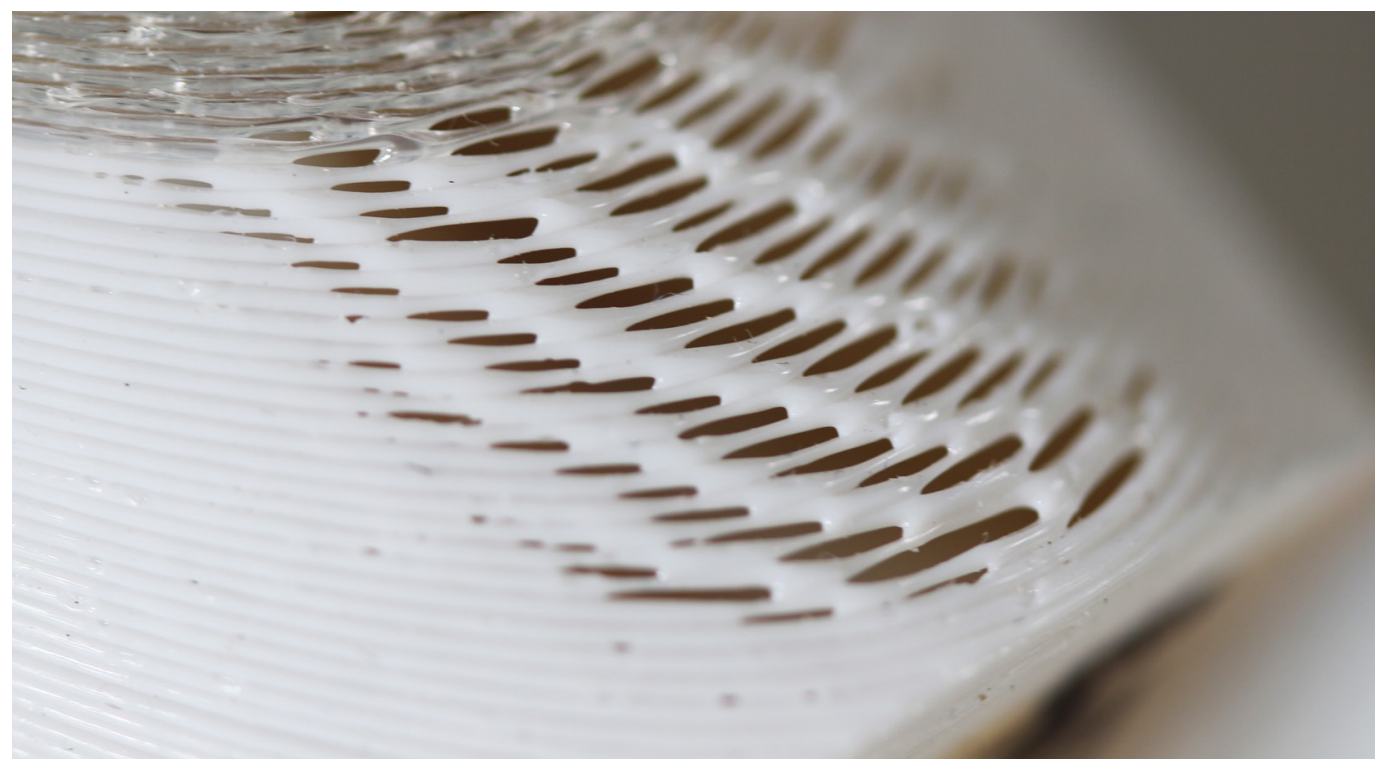

Figure 27 - Patterning created from the PLA white plastic.

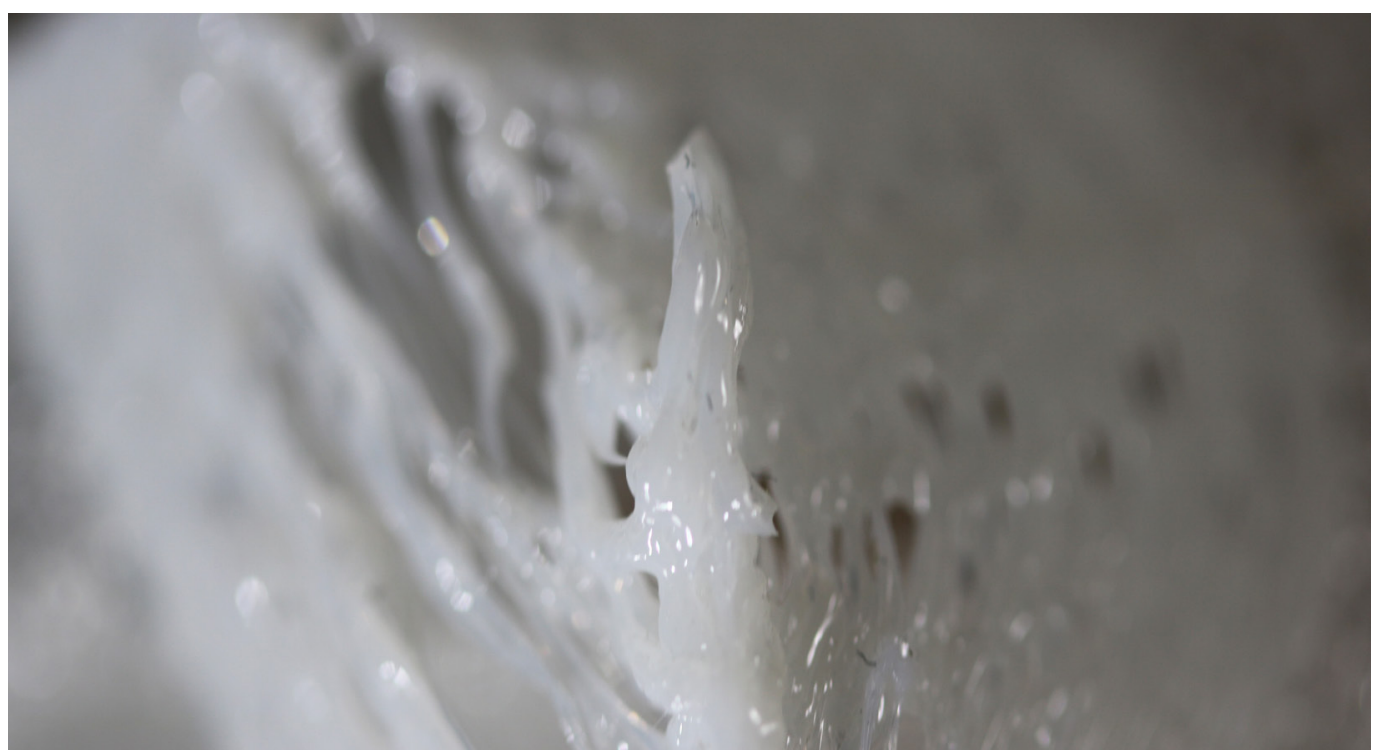

Figure 28 - Buildup of TPU plastic created at the tip of the model. 


\section{APPLICATION}

\section{Cantilever Seating}

By combining the results from the initial material experiments and using the Panton Chair as a precedent, an exploration into cantilever seating was established. Sketching and modelling chairs commenced an investigation into how these two materials, PLA and TPU plastics, could improve chair design by taking advantage of their individual material qualities.

The small scale material experiments took advantage of how the printer operated. By printing the seats on their sides encouraged a higher quality final object using a single path of material. This made it easier for the printer to construct the chair without support while providing good adhesion between layers. The chairs are designed to allow the wooden material to flex under compression, as it does with some traditional cantilever seating. Large overlapping areas of flexible TPU increase the strength and support of the cantilevered structures.

These initial seating tests explored the aesthetics of seating as well as manufacturing orientations. Having a seat that is $3 \mathrm{D}$ printed can allow for unique aesthetics that are unable to be achieved using traditional manufacturing techniques. Orientating the seat onto its side during printing creates different build qualities when compared to printing it in an upright orientation.

At this stage there is no incorporation of $3 \mathrm{D}$ scanning. This allows the seat to be customised to fit an individual's form. The incorporation of this technology is essential to the thesis aim and influence of application and context.
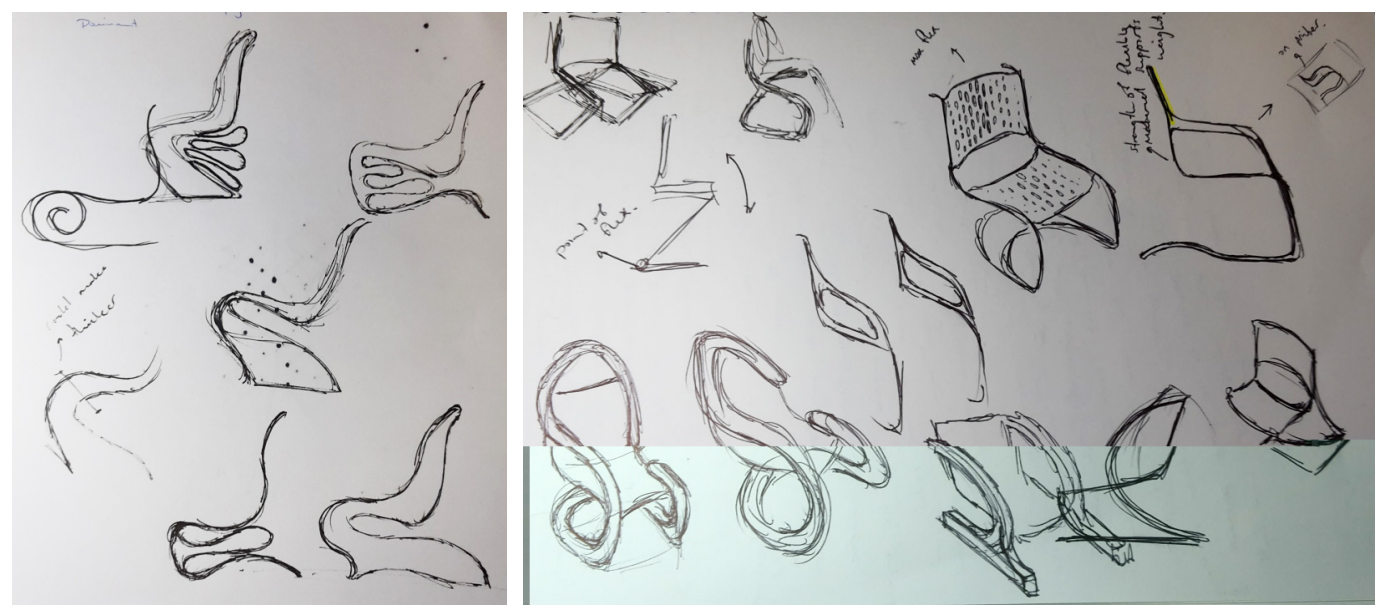

Sketching concept ideas of cantilevered seating. 

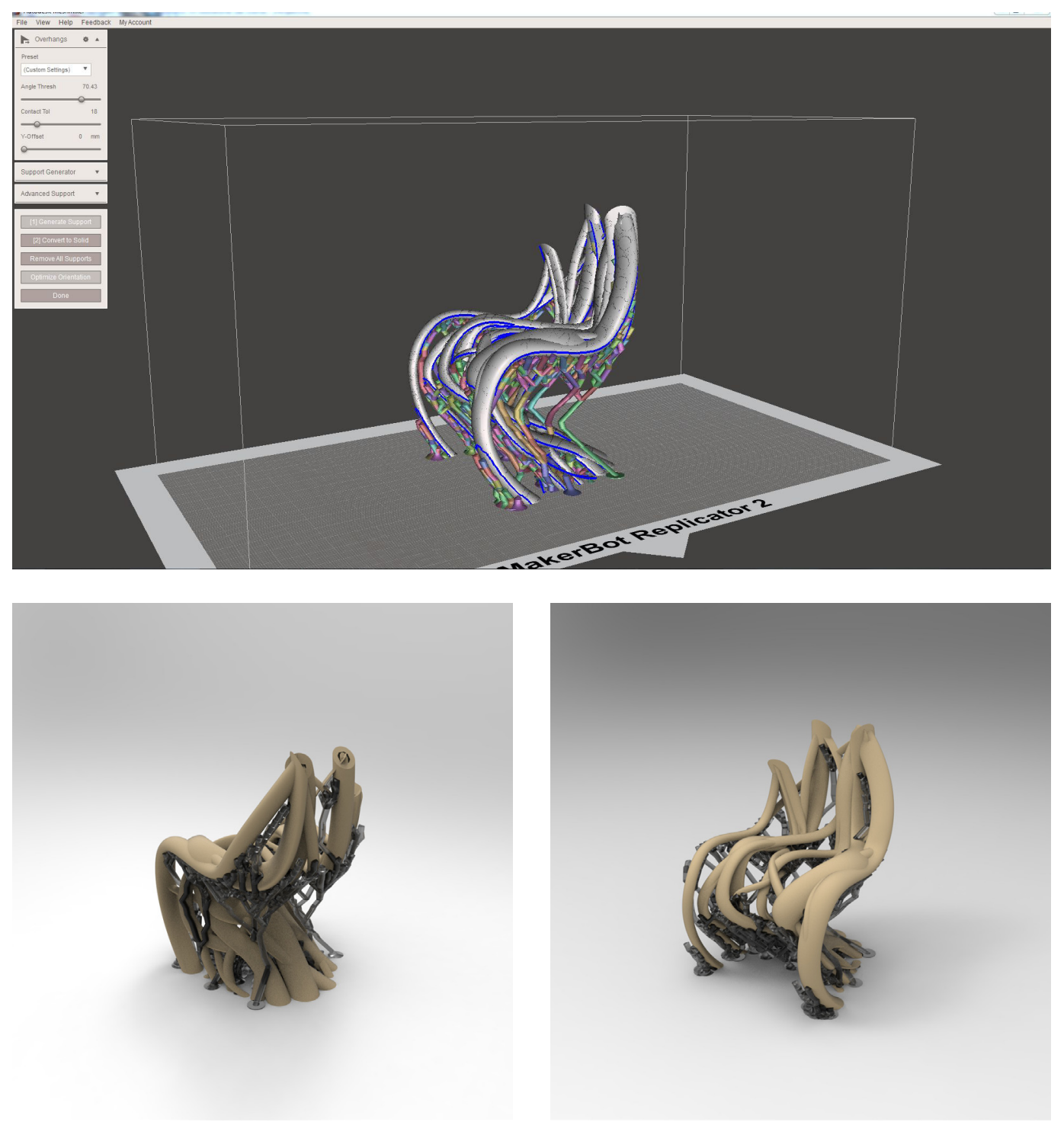

CAD Models explore how 3D printed support material can be designed to be a part of the model rather than being removed through post processing.
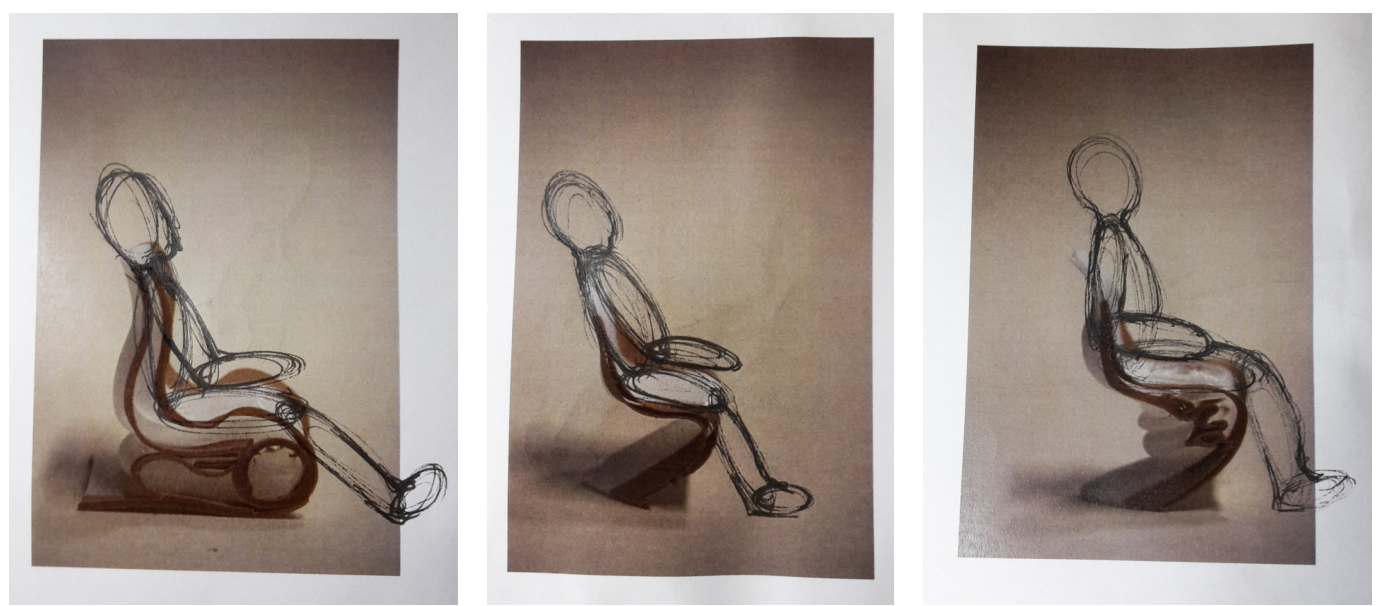

Gaining an understanding of how a person may fit to the full scale prototype. 

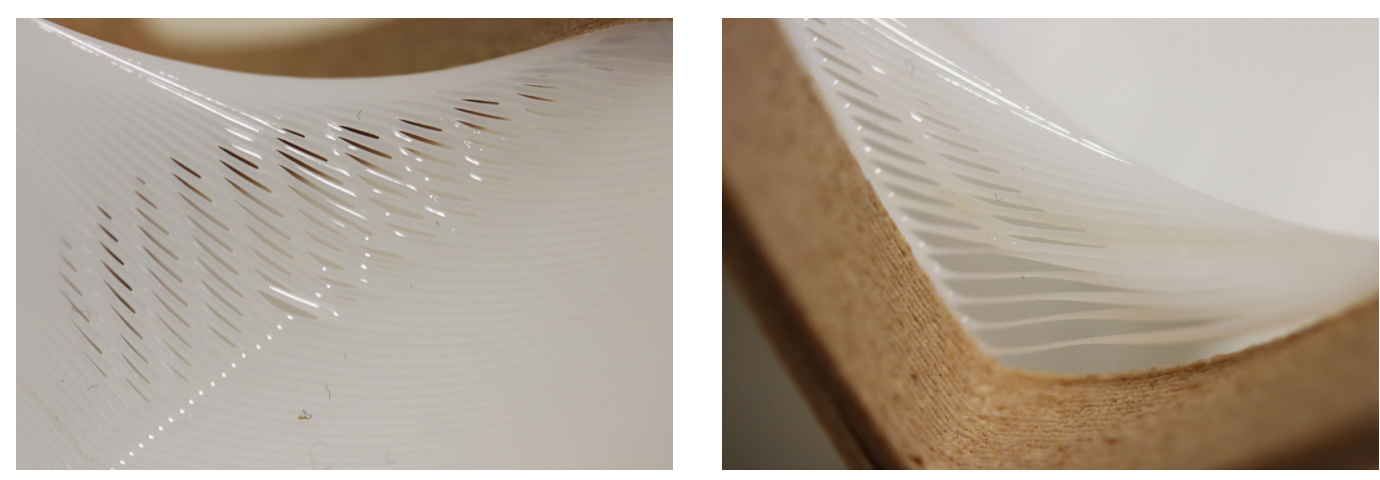

Patterning is created by the movements of the print head extruding the TPU material at an angle.

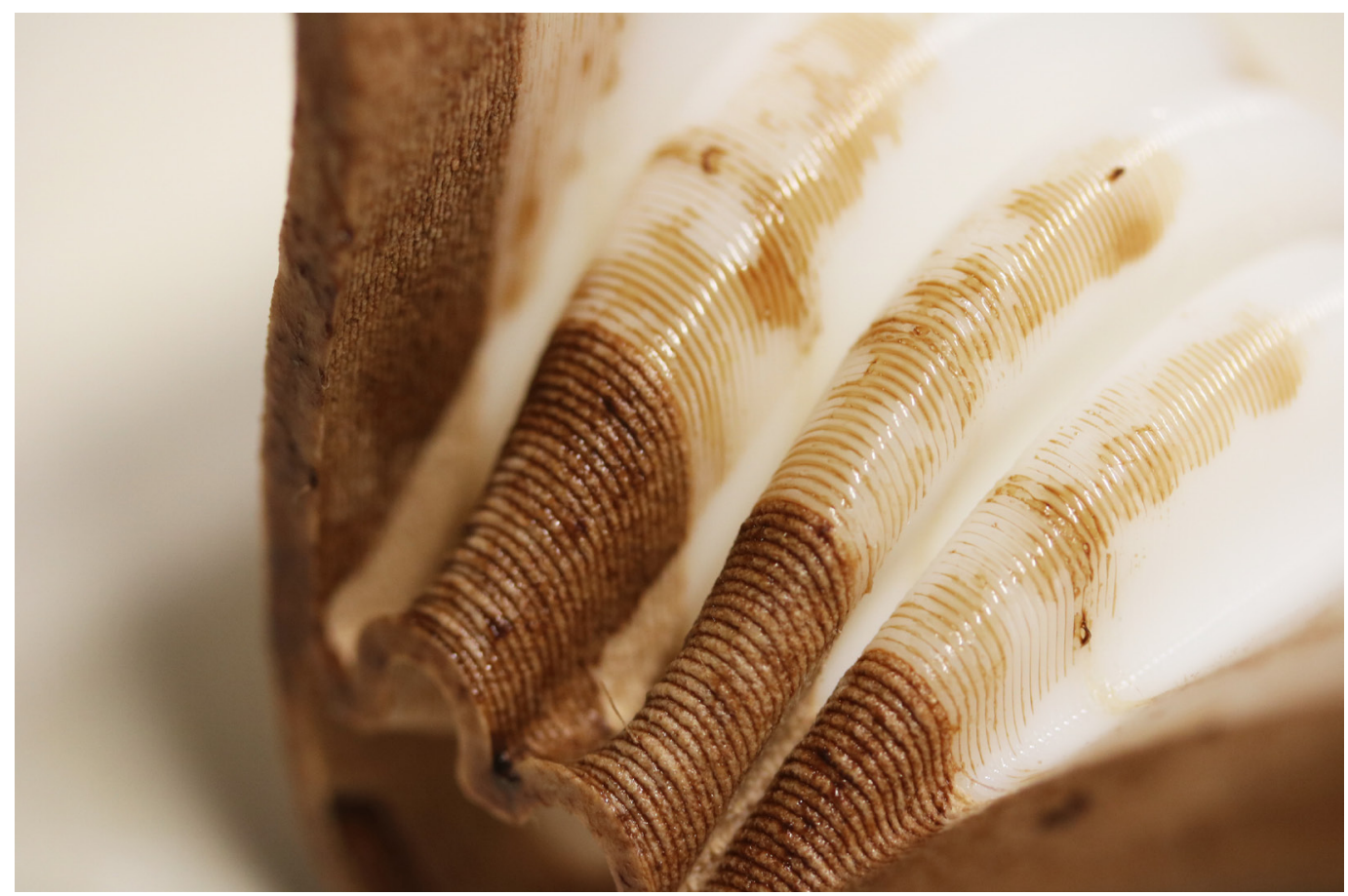

The PLA wood material within this model has been treated with wood stain. This is an experiment exploring how the final model may be post processed. 

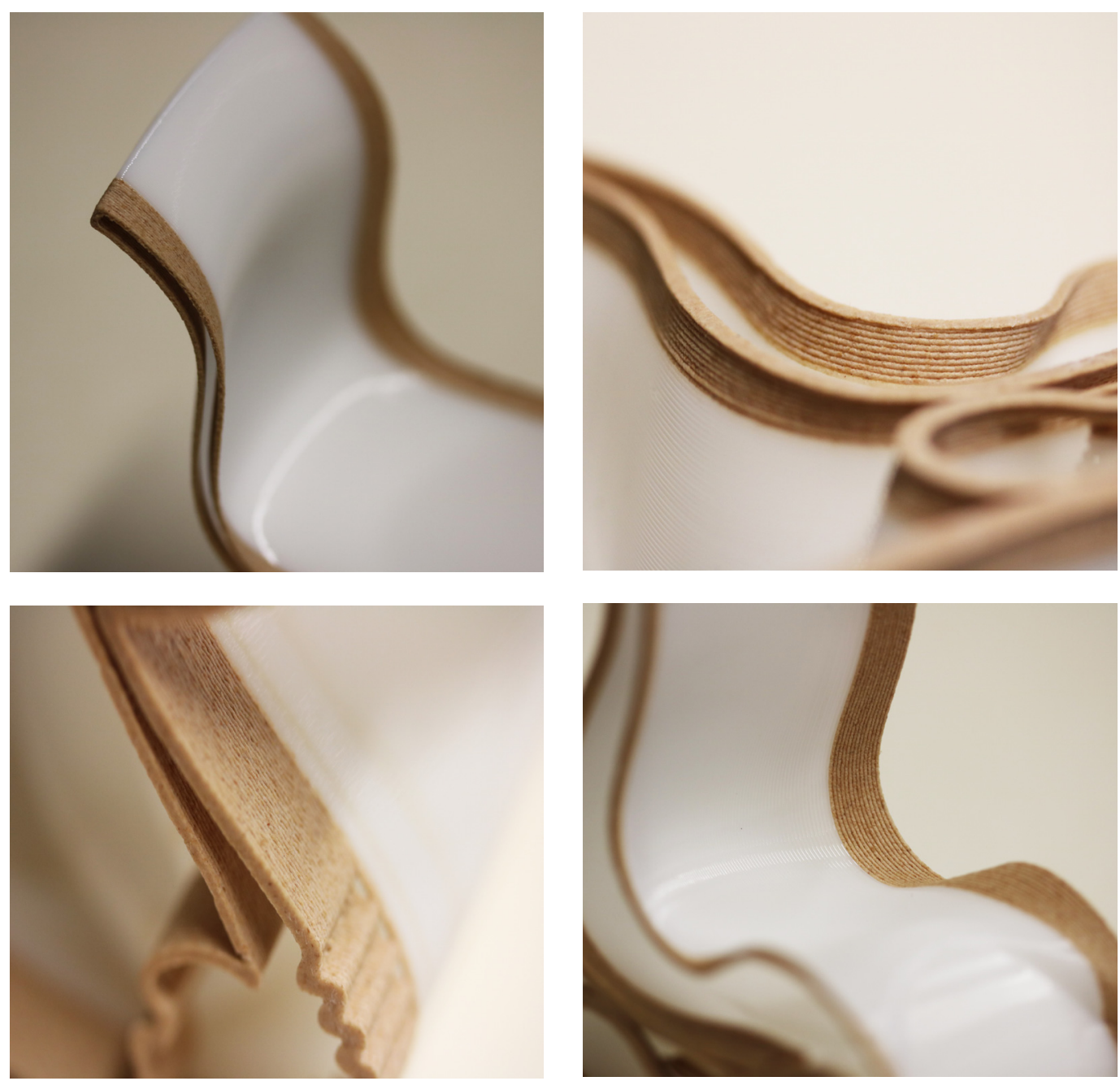

The layer pattern within these models is created by the chairs being 3D printing on their side. Rather than hiding this pattern, it helps add to the aesthetic of the chair.
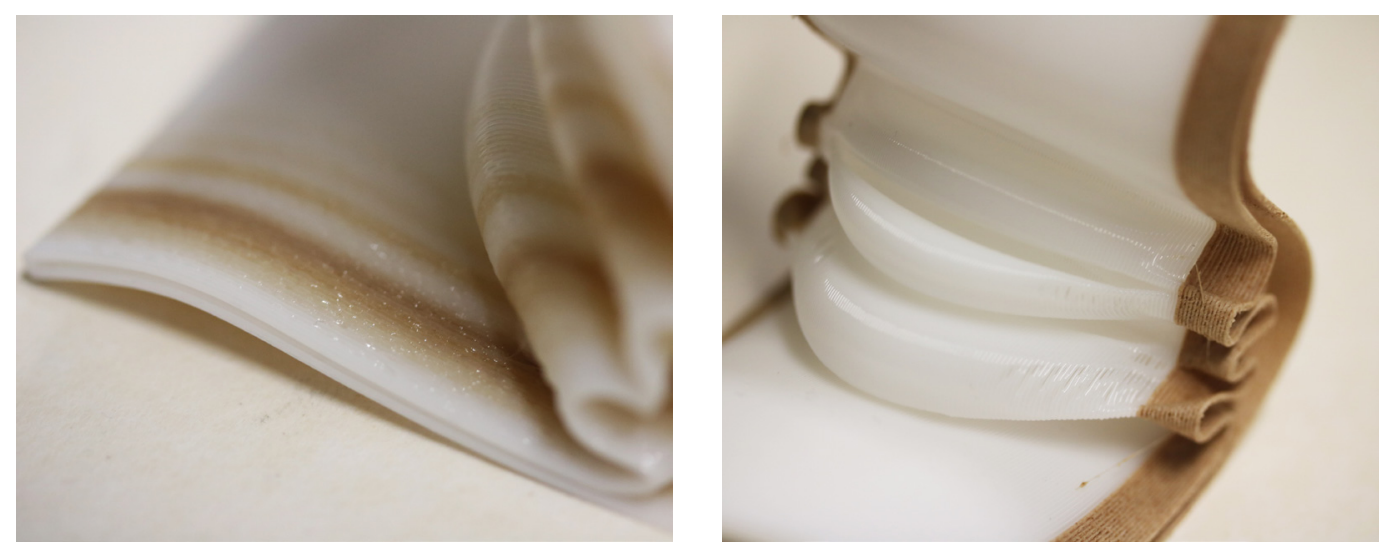

These overlapping areas are designed to compress under load. this gives the person's body support when sitting on the chair. 
On reflection of the progress made in understanding FDM 3D printing, and the combination of materials, a potential design and application was sought.

Whilst the Panton Chair offered a suitable methodological approach and a demonstration of human scale 3D printing, it lacked personalisation through scanning. Some concepts of personalisation to particular people through scanning were sketched up. However, an application more aligned to an established product and recognised problem was identified.

Motorcycles are generally, solely ridden by an individual. This justifies the utilisation of a custom seat designed to their form.

The fact that motorcycles are designed for a broad percentile has been established. This creates an opportunity to design the seat of the motorcycle, to not only personally fits the form of any rider who fits outside of this general percentile, but also provide a customised level of support based on the rider's proportions.

Many motorcycle seats are considered hard and extremely uncomfortable to use over an extended distance of riding. Results from a survey that focused on customised products, concluded "that the majority of motorcyclists felt discomfort during long distance travelling and that a customised motorcycle seat that exactly fits to the body geometry appeals to the majority of motorcyclists surveyed" (Ong, Wagner, Tuck, \& Hague, 2008, p.375). This article also reviews the higher price and wait time riders are willing to tolerate when acquiring customised products. It is evident that the desire for customised products is apparent and by using 3D printing and 3D scanning technologies, an individually designed motorcycle seat has the potential to solve this problem. "
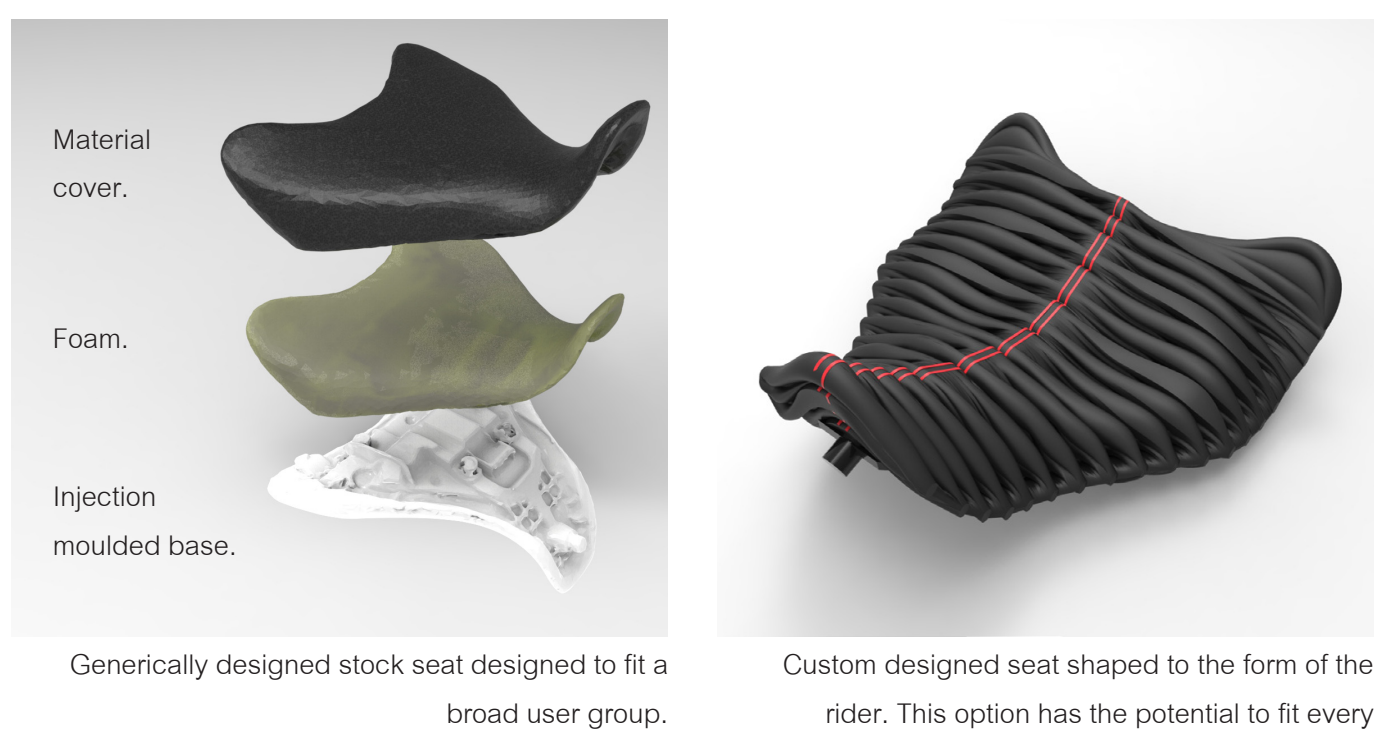

user not just a select percentile. 


\section{Support Testing}

It is intended that the application of motorcycle seating will be constructed using 3D printing. The seat aims to be able to support the weight of a person within a reasonably thin physical area, fitting the aesthetic of the motorcycle as well as to the rider's dimensions. Designing vertical tubes which compress underweight was an idea to create 3D printed cushioning.

The first trial compresses unevenly making it difficult to design intended weak compression areas (Figure 29). When turning this original shape onto its side, it created a more successful cushioning system (Figure 30).
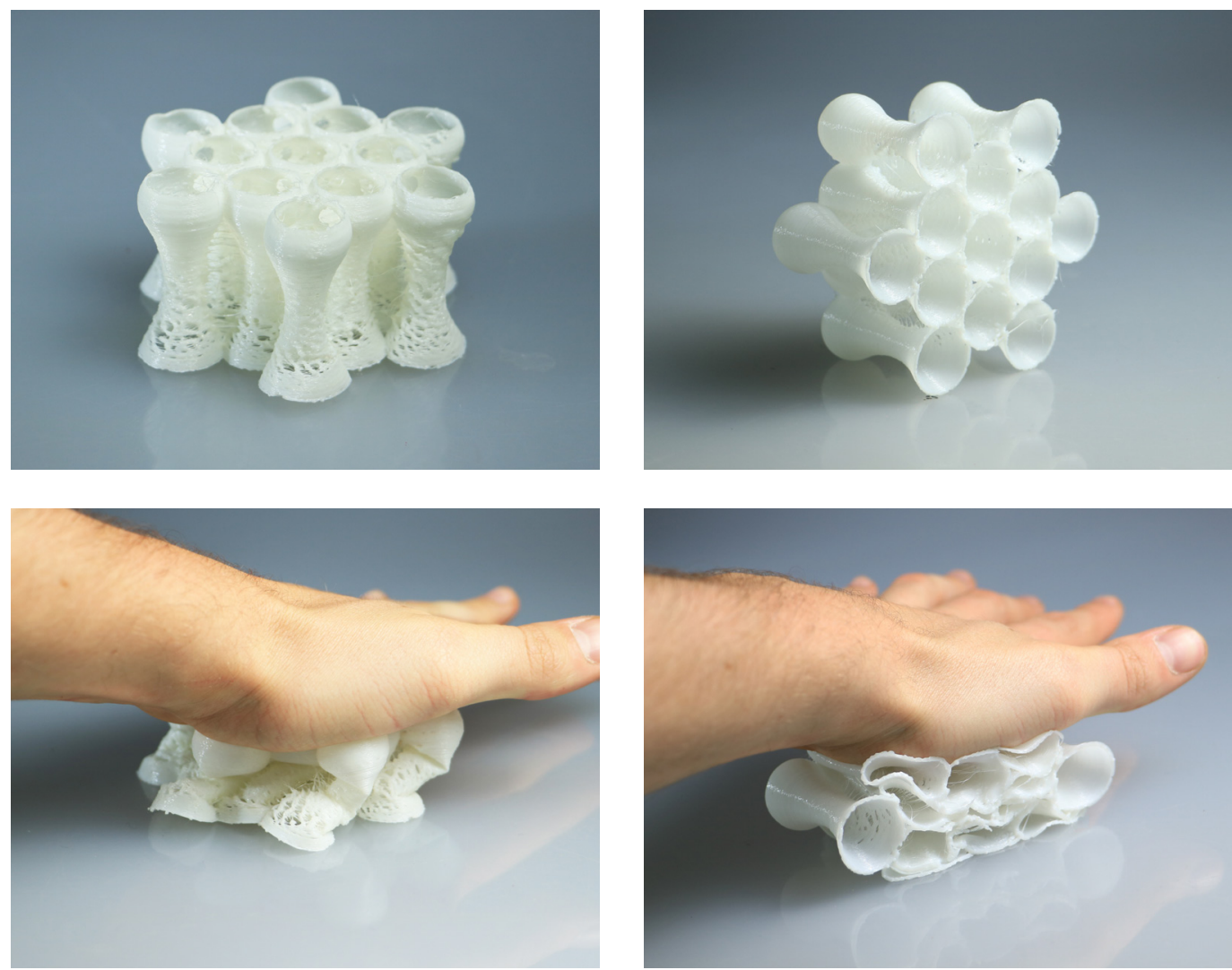

Figure 29 - Uneven compression of support structure.

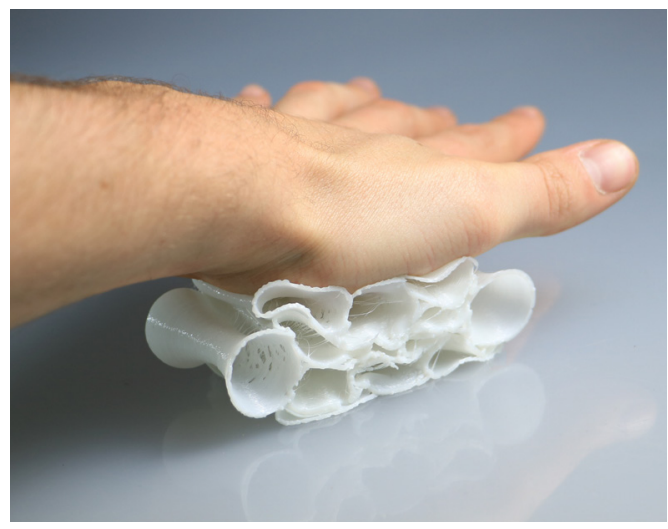

Figure 30 - The load is evenly supported.
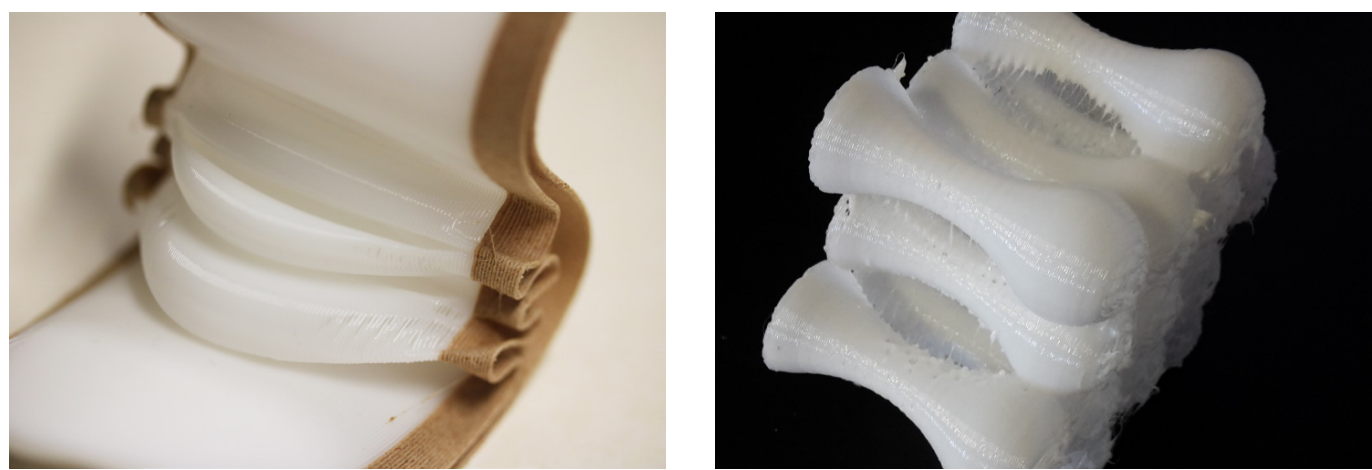

Tubular support tests, derived from the overlapping areas, designed in the cantilever seating experiments, show a promising result when compressed. This idea was carried over to the motorcycle application, to give the rider support when sitting on the bike. 
A series of structure tests explored a variety of wall thicknesses and the ratio of supporting tubes to free area. This lead to creating different levels of support and structure constructing a range of cushioning options.

Smaller test structures illustrated how the cushioning would work as a seat, however unlike the previous TPU and PLA trials, these structures acted very differently when more tubes were added or scaled. Larger support structures were therefore created to increase the real world accuracy of how these structures would support a body at full-scale.

These cushioning tests have created opportunities to provide comfort using the TPU plastic, through a combination of physical design and material qualities. Using these tubular structures has allowed for an interesting aesthetic which is different from the traditional, upholstered seating design people are used to.
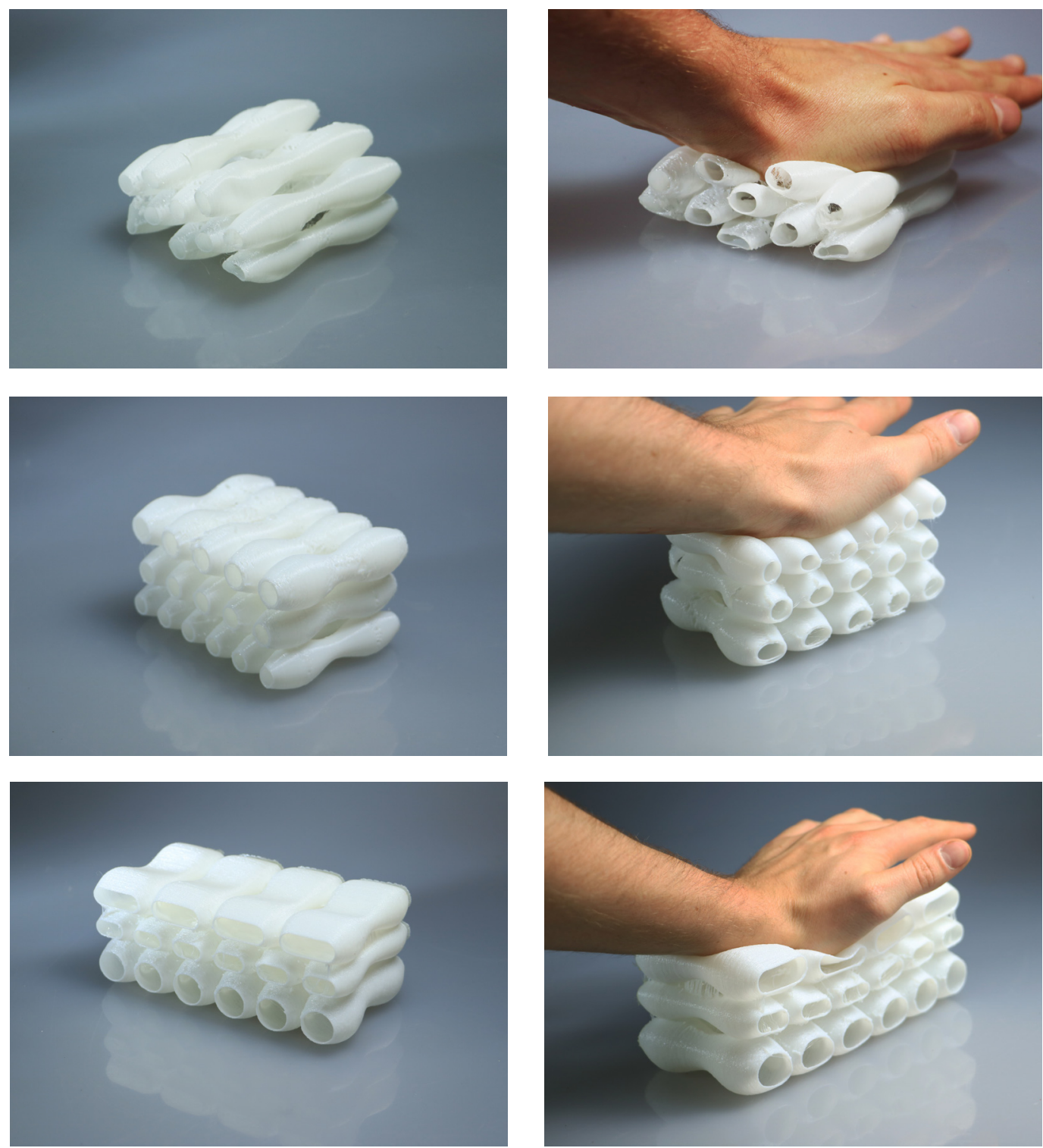

Support structure testing. 


\section{APPLICATION}

\section{D Scanning}

3D scanning is used to gain the rider's digital geometry. To get a positive 3D scan of the rider in a weight bearing riding position, a negative cast of the rider's form was made while they sat on the bike.

The method of how the cast is moulded is as follows:

1. A protective fabric sheet is put across the top of the motorcycle to stop it from getting scratched and damaged by the polyethene sheet. On top of this, a polyethene sheet is laid to form a barrier from water and plaster

2. The participant will put on disposable clothing and their standard riding footwear. The footwear will be covered in large plastic bags to ensure they stay clean and undamaged.

3. Plaster of Paris strips are soaked in water and placed onto the polyethene covered seat area of the motorcycle. The $30 \mathrm{~cm}$ plaster strips are laid out over the area of the seat until it is covered. Multiple layers of plaster strips are added to give a solid thick layer of plaster casting.

4. The participant will sit on top of the motorcycle and assume their natural riding position, with their hands on the grips and their feet on the pegs.

5. The rider will be asked to stay in this position for approximately 25 minutes letting the plaster harden enough to hold the shape of the participant.

Once the plaster cast has dried a 3D scan is made of the participant's impression.

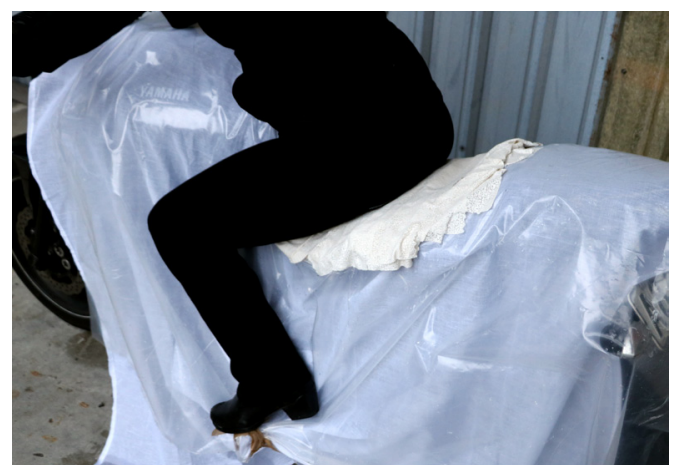

3D cast of the riders form

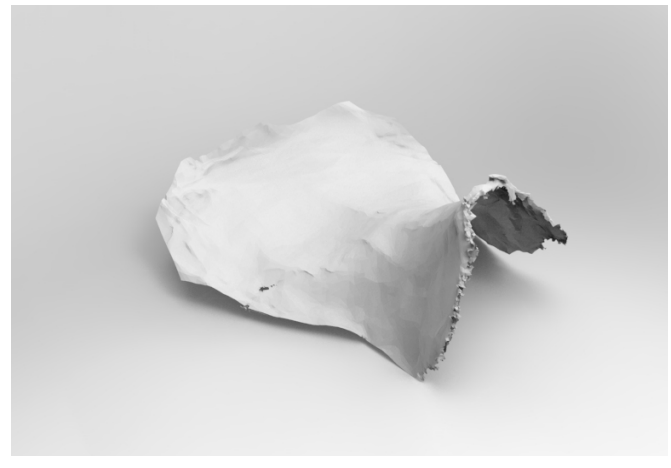

3D scan of the riders form. 


\section{Full Scale Prototyping}

The support test models revealed that tubular structures gave appropriate support when designed to $20 \mathrm{~mm}$ diameters. Dividing the scanned casting into $20 \mathrm{~mm}$ sections allowed the previous tubelike structures to be designed using horizontal form lines (Figure 31). Being horizontal allows the seat to be 3D printed on its side, ensuring the maximum quality with as little overhanging areas as possible and without any interior support structure. Using the form lines created a seat that physically fitted the rider as well as allowing for varied thickness and support within the seat. \

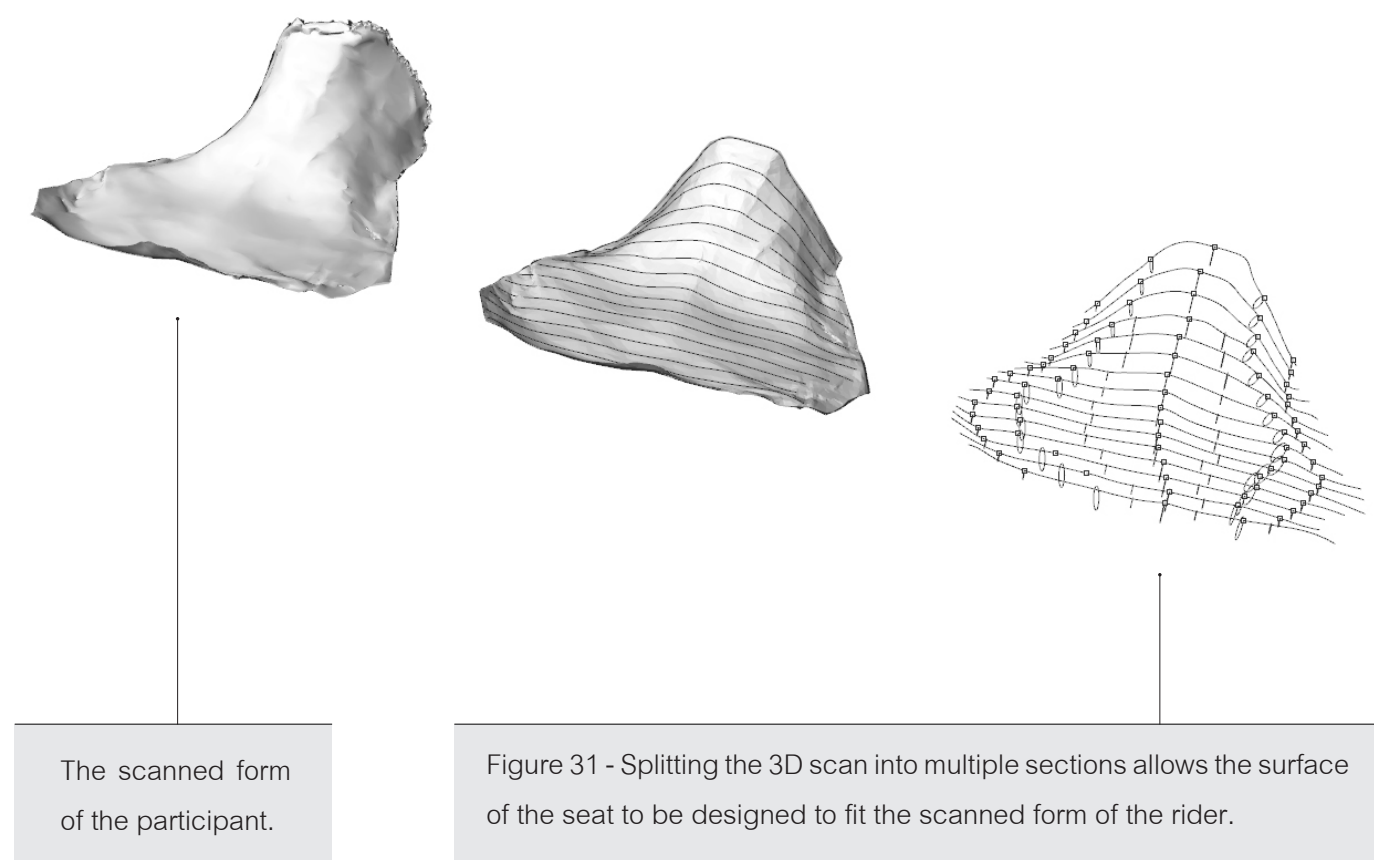




\section{Pressure Measurement System}

A pressure pad was used to provide digital feedback on the amount and concentration of the force exerted onto areas of the seat. This pressure pad was easy to use as it was draped over the seat and then sat on in a natural riding position. This allowed for a digital pressure evaluation of each prototype.
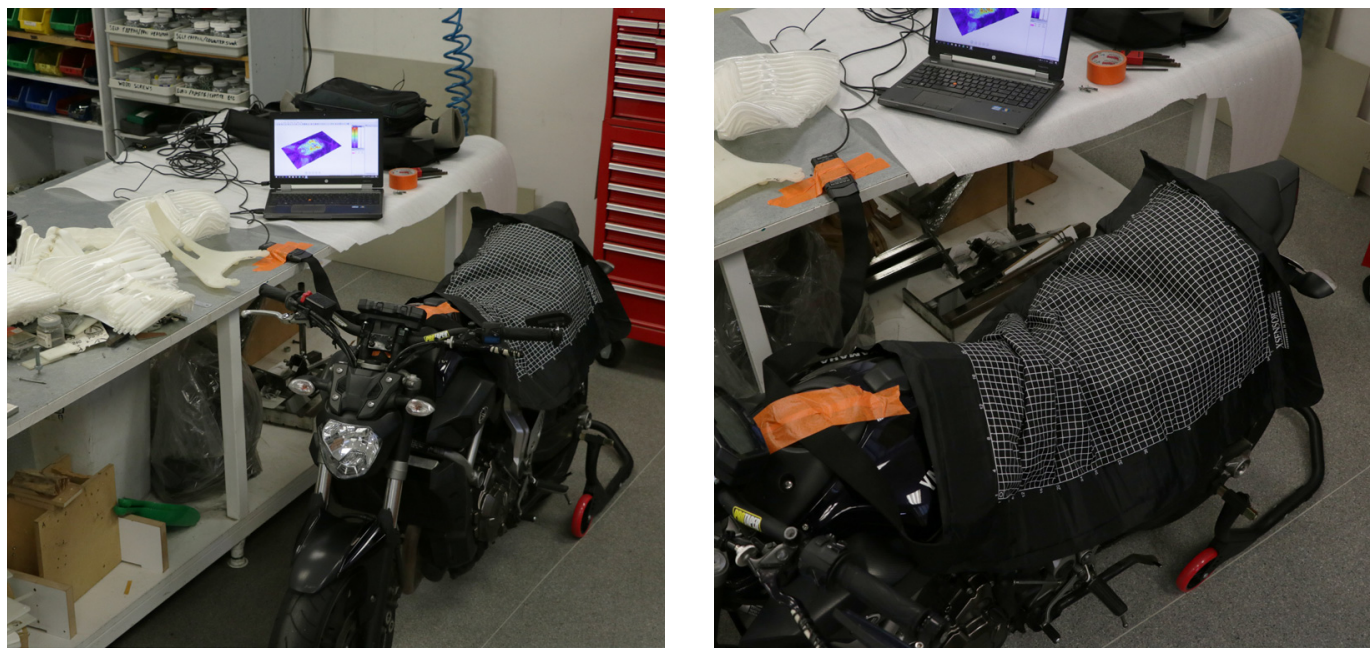

The computer evaluates the data the pressure pad receives, displaying it as a live video.
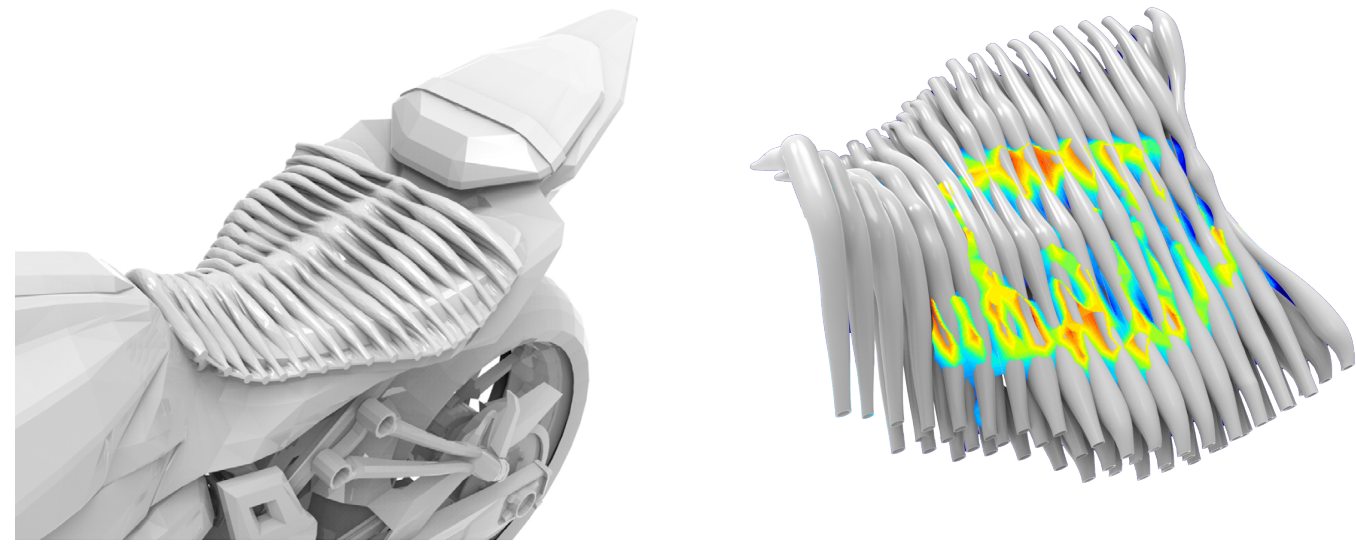

The data received from this system has been overlaid onto each of the seating prototypes visually representing how the rider's weight is distributed over the surface of each seat. 


\section{Support Base}

The support base is the rigid structure within the seat that connects to the motorcycle frame. This is $3 \mathrm{D}$ printed in PLA plastic and holds the prototype seat in place. The seats are currently printed in separate mono material prints and tethered together with the support base for testing.

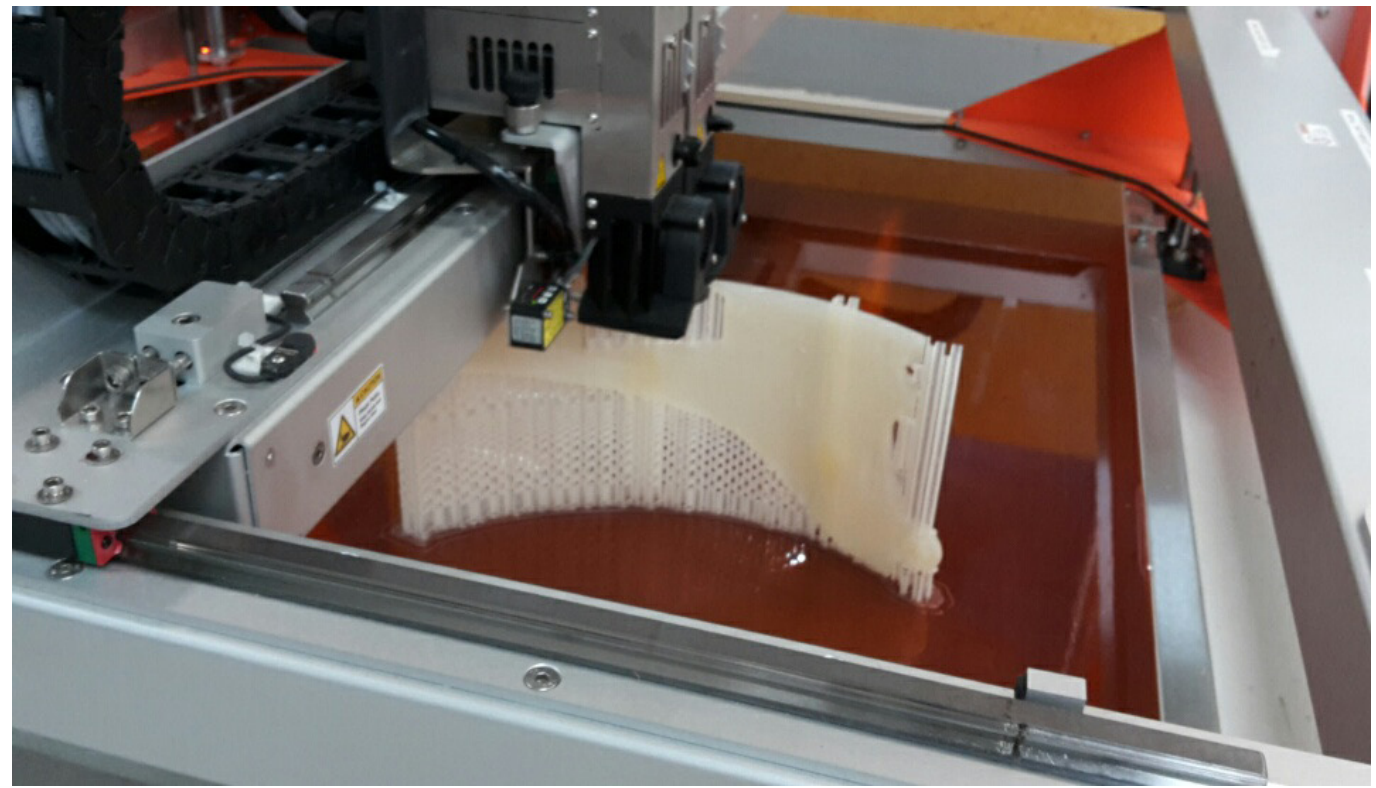

Two stages of the hard base being 3D printed.

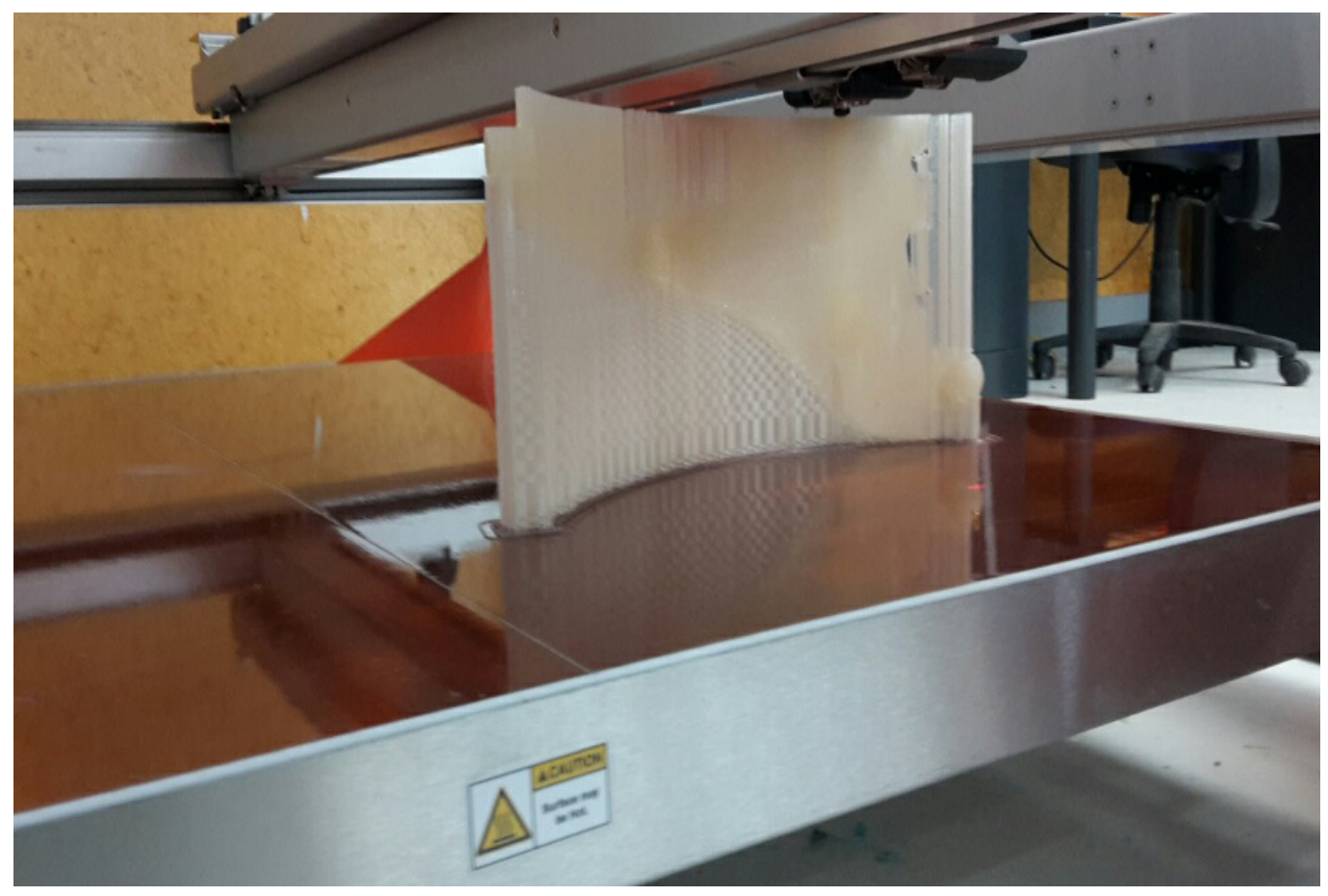



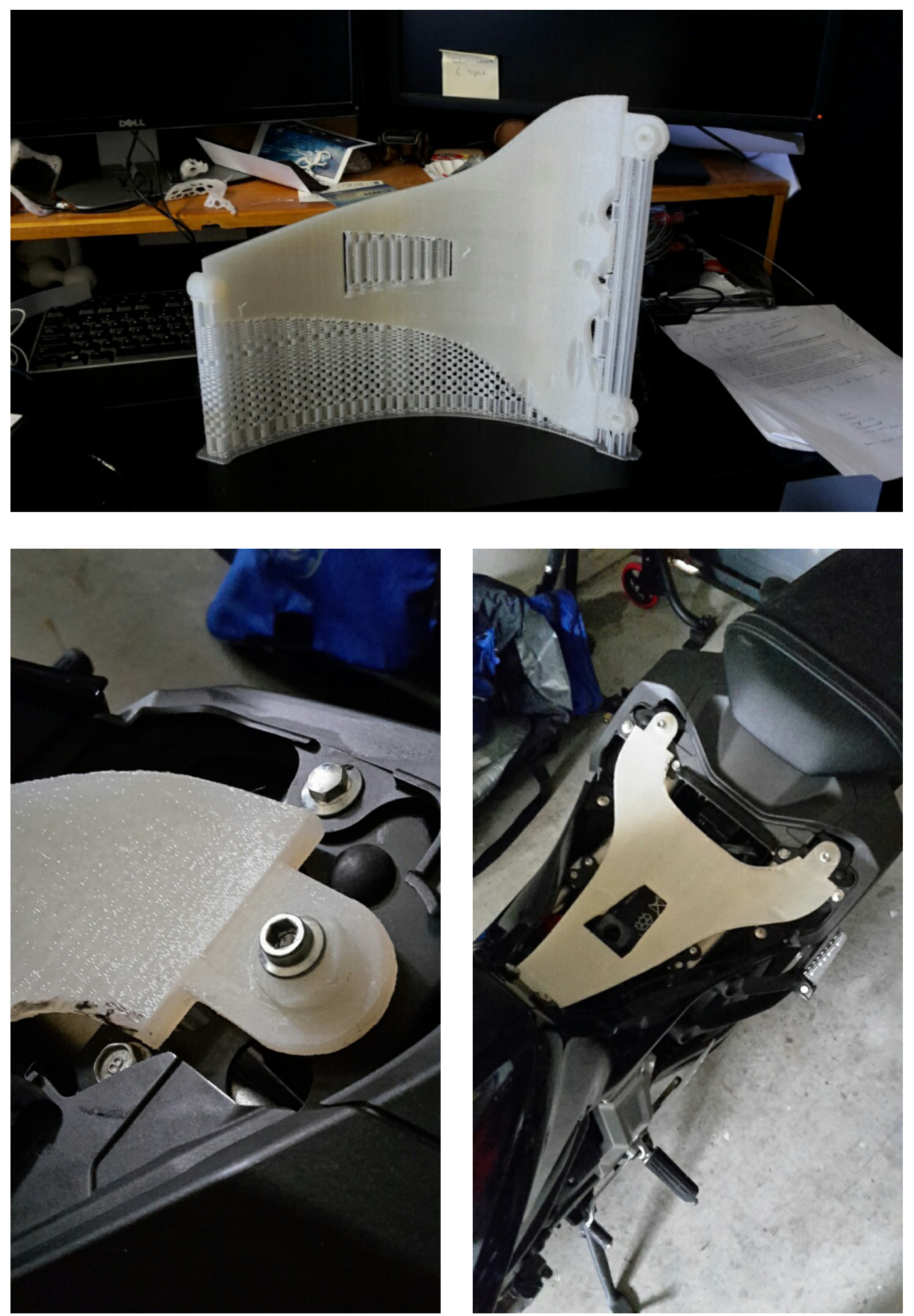

The hard base is bolted to the motorcycle frame thus providing a solid support for the prototype seats. 
The first set of full scale seating prototypes explored the use of the riders scanned form. These prototypes set out to test an initial theory of personalised motorcycle seating at 1:1 scale.

Prototype 1 was mono-material 3D printed with a single layer wall thickness. The rider could feel a noticeable difference between the stock seat and the seat that was designed to the individual rider's body. Figure 32 illustrates the areas on the left-hand side of the seat which required more support.

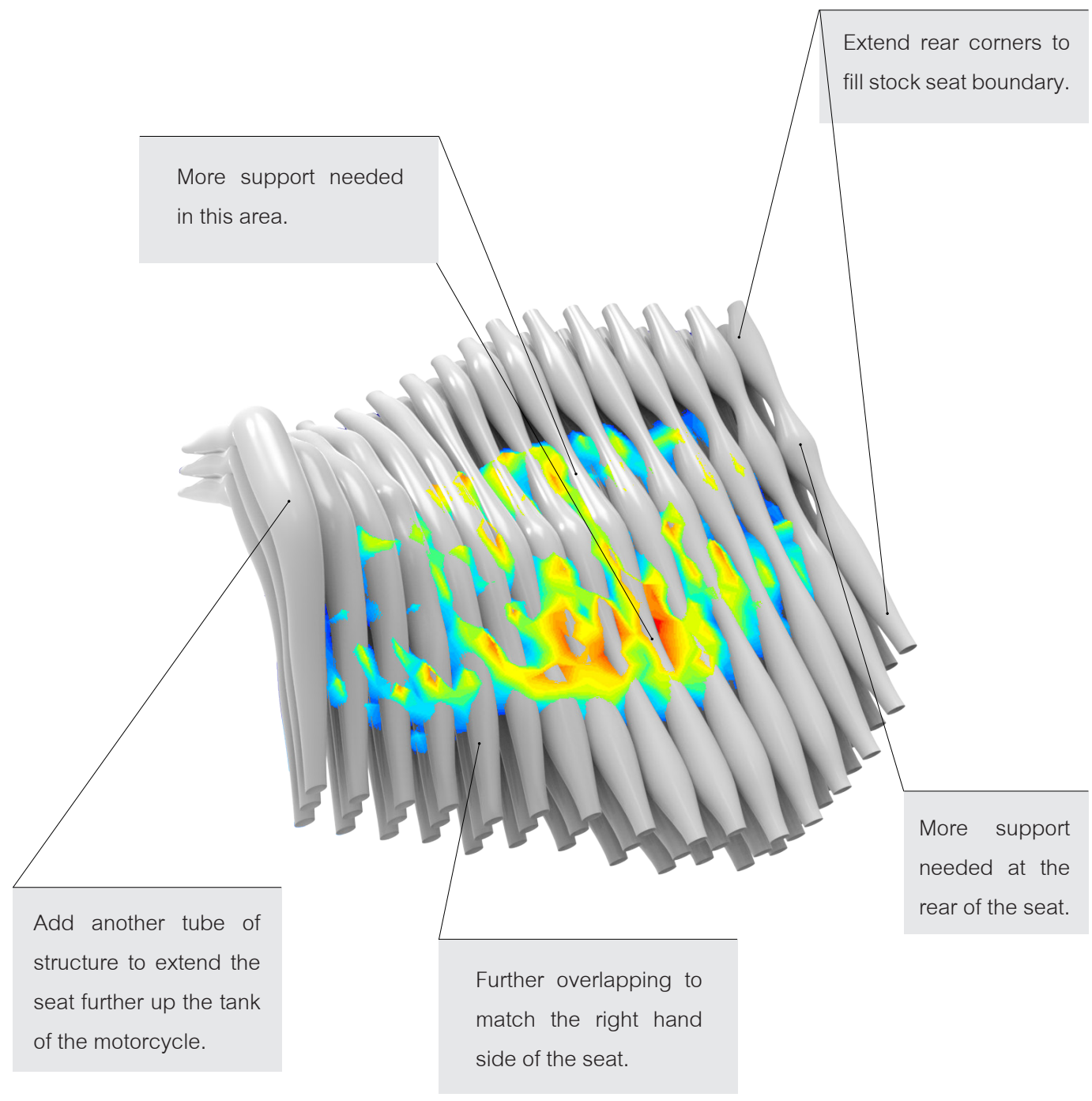

Figure 32 - Prototype 1 

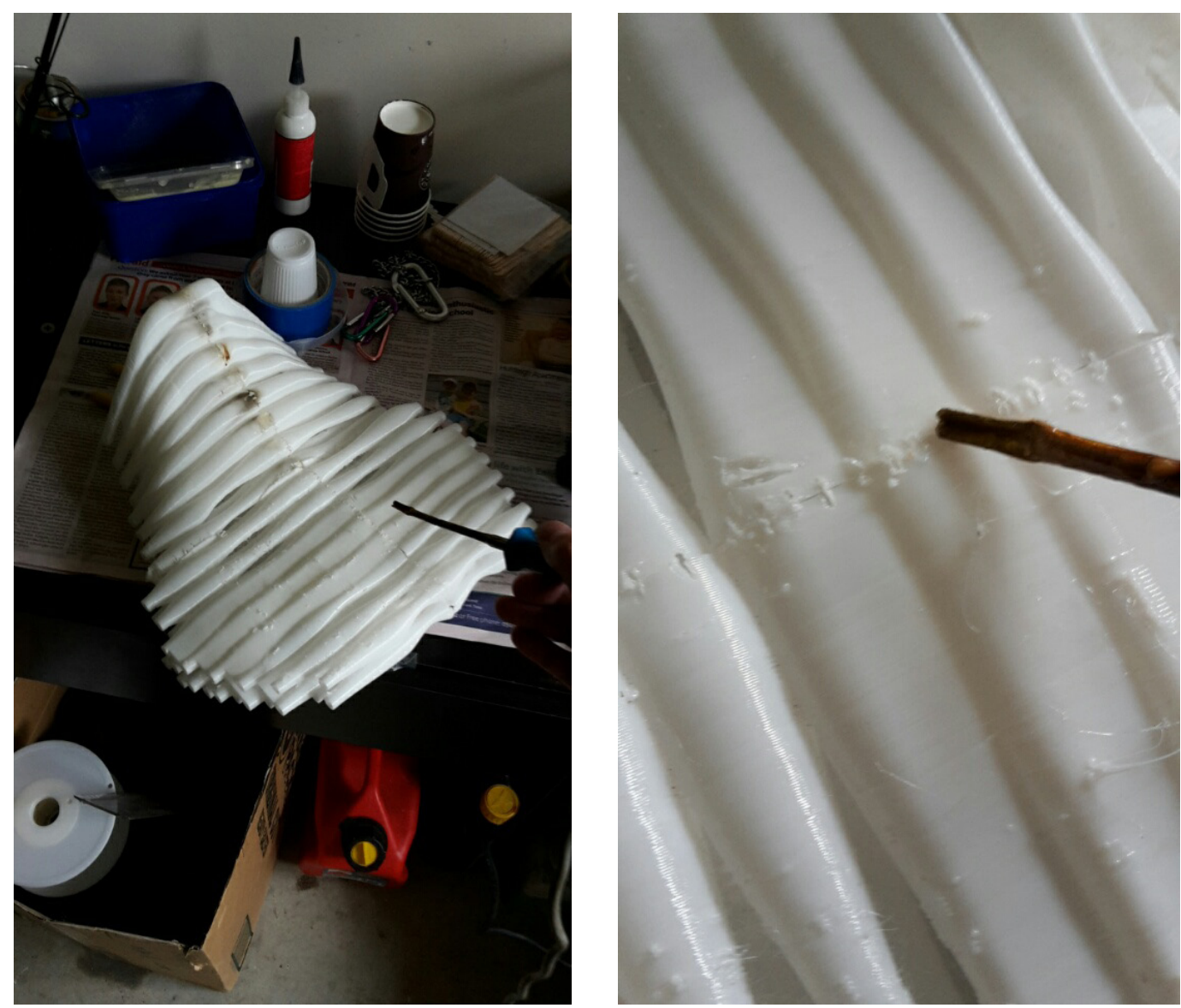

This prototype is made up of individual prints which are tacked together using a soldering iron.

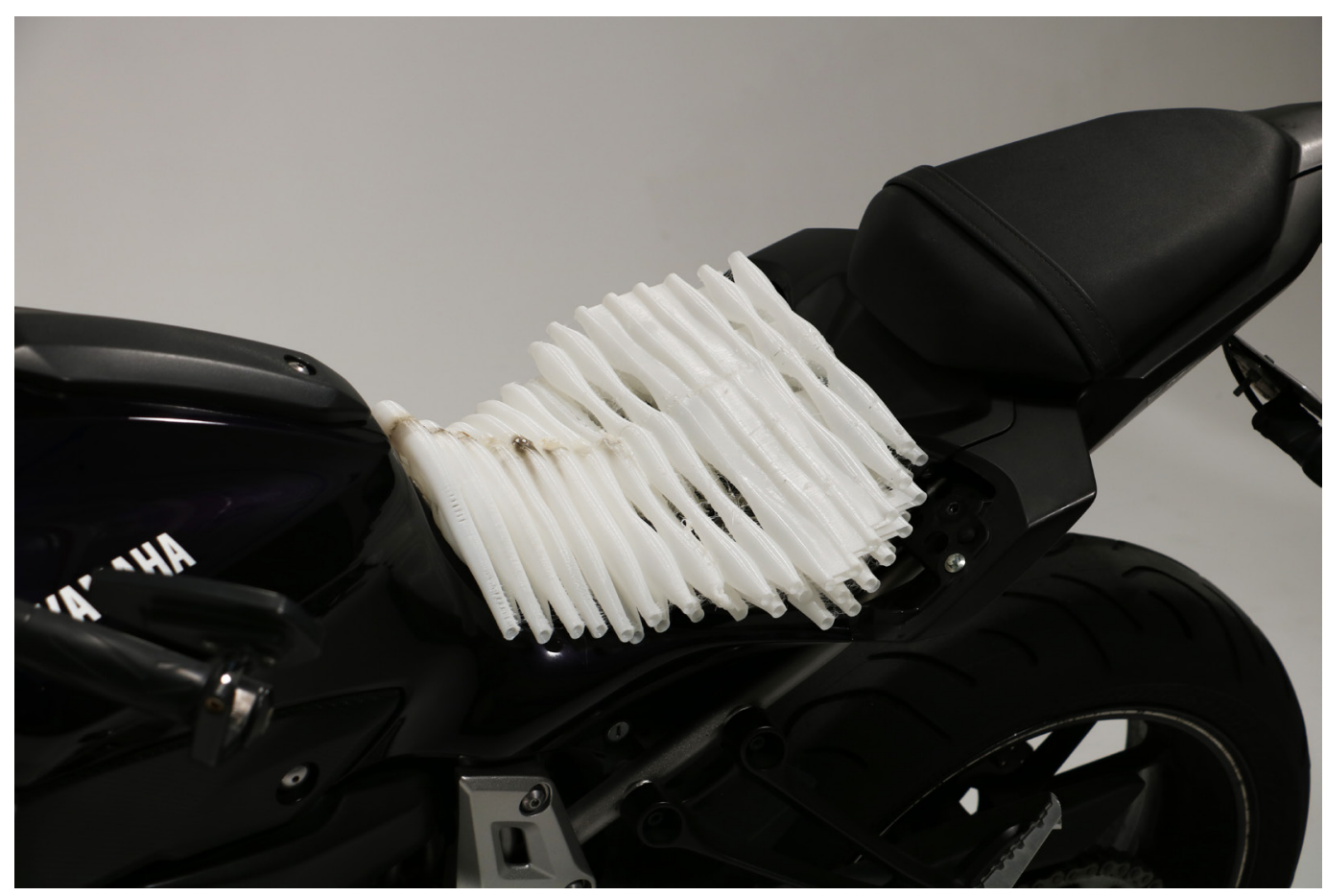

Prototype 1 mounted to the motorcycle. 


\section{Prototype 1, 2, 3}

The previous prototype lacked support which increased the overall thickness of Prototype 2. Figure 33 indicates reduced pressure on the left-hand side of the seat, however the increase in material thickness made it hard and uncomfortable for the rider to use. The rear of the seat was extended to fit the available seat space on the motorcycle.

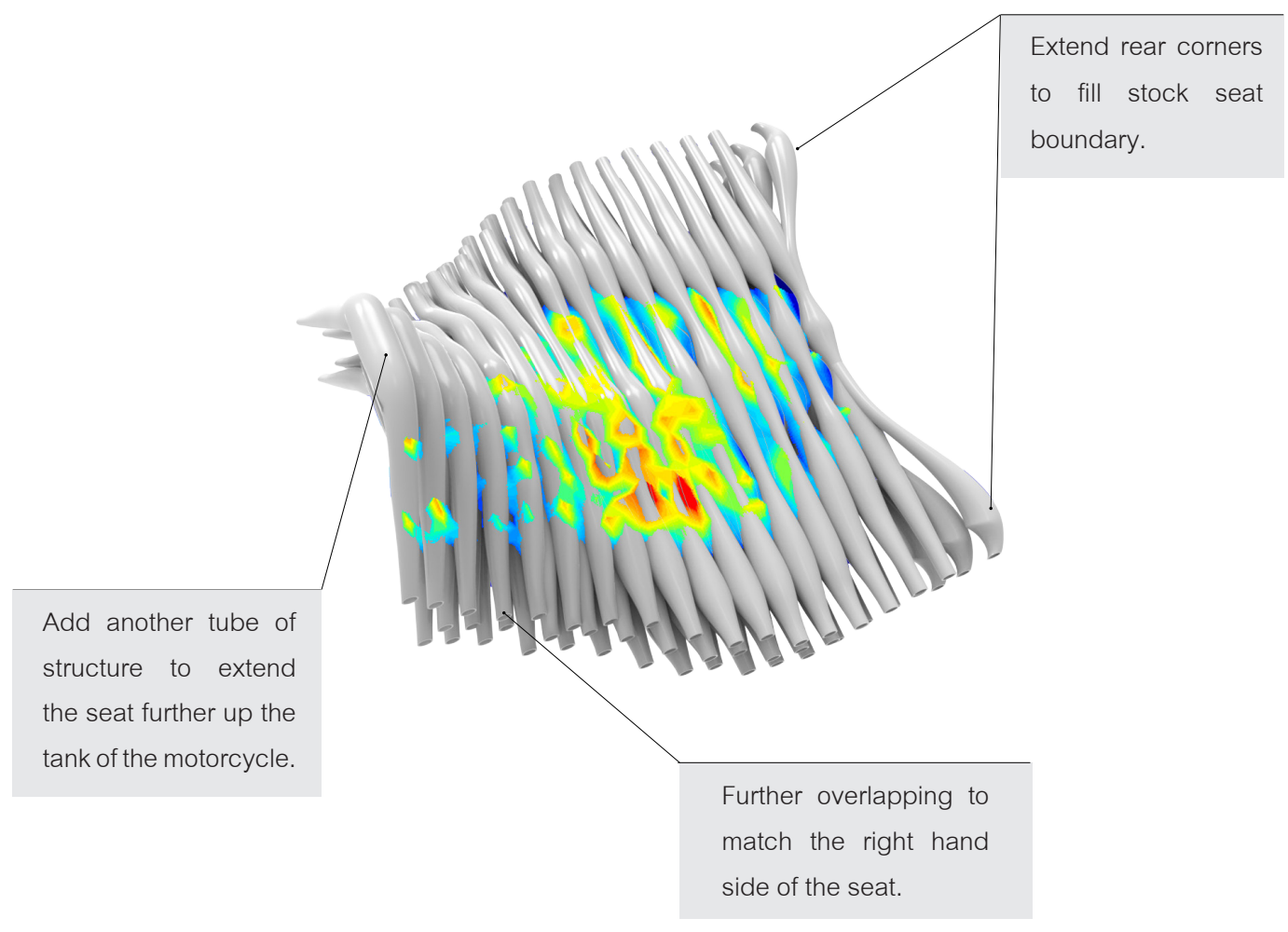

Figure 33 - Prototype 2

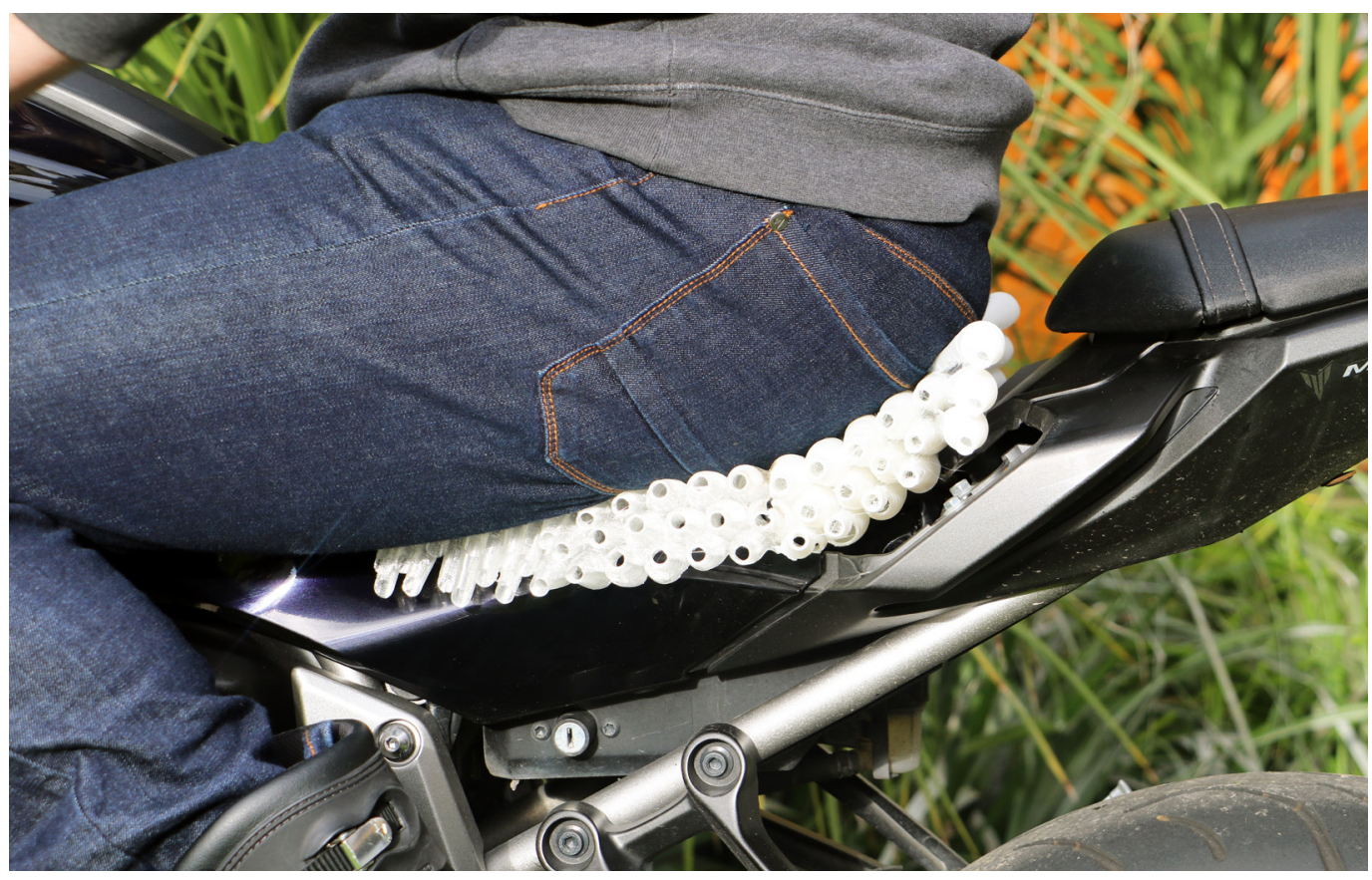


Prototype 3 had been 3D printed with a double wall layer. It provided a good overall level of support, however Figure 34 shows an increase in pressure at the front of the seat which reduced comfort levels. This same pressure point was noticeable for the rider when testing. This created discomfort soon after sitting on the seat. The profile of this prototype was remodelled to reduce the specific pressure points.

\section{REFLECTION:}

This set of initial full scale seating prototypes have introduced the rider's form and material structure to the design of a motorcycle seat. These seats have created a bridge between the generic shape of the motorcycle and the individuality of the rider's form. The next set of prototypes will focus on the aesthetic and how the seat interfaces with the motorcycle.

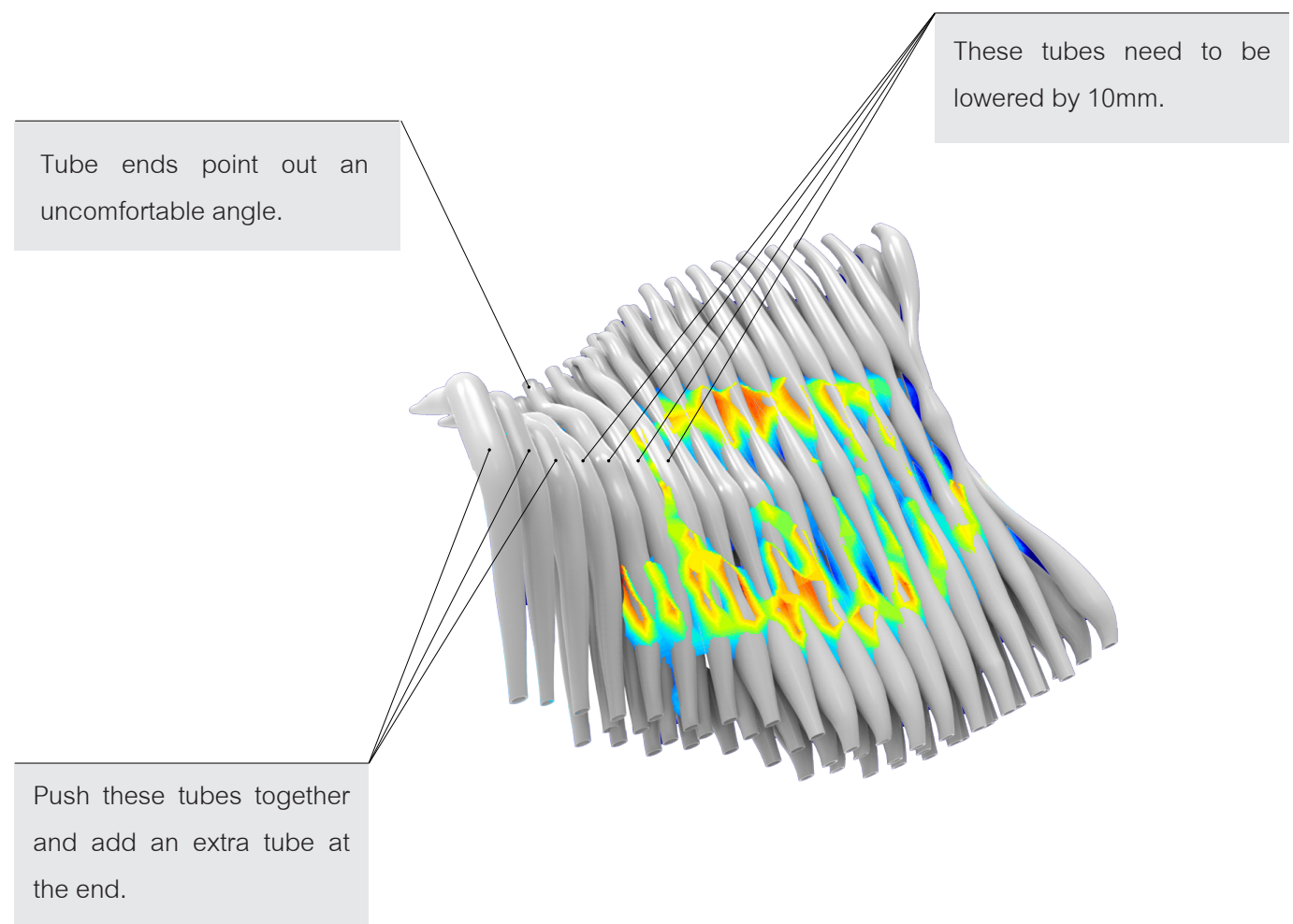

Figure 34 - Prototype 3

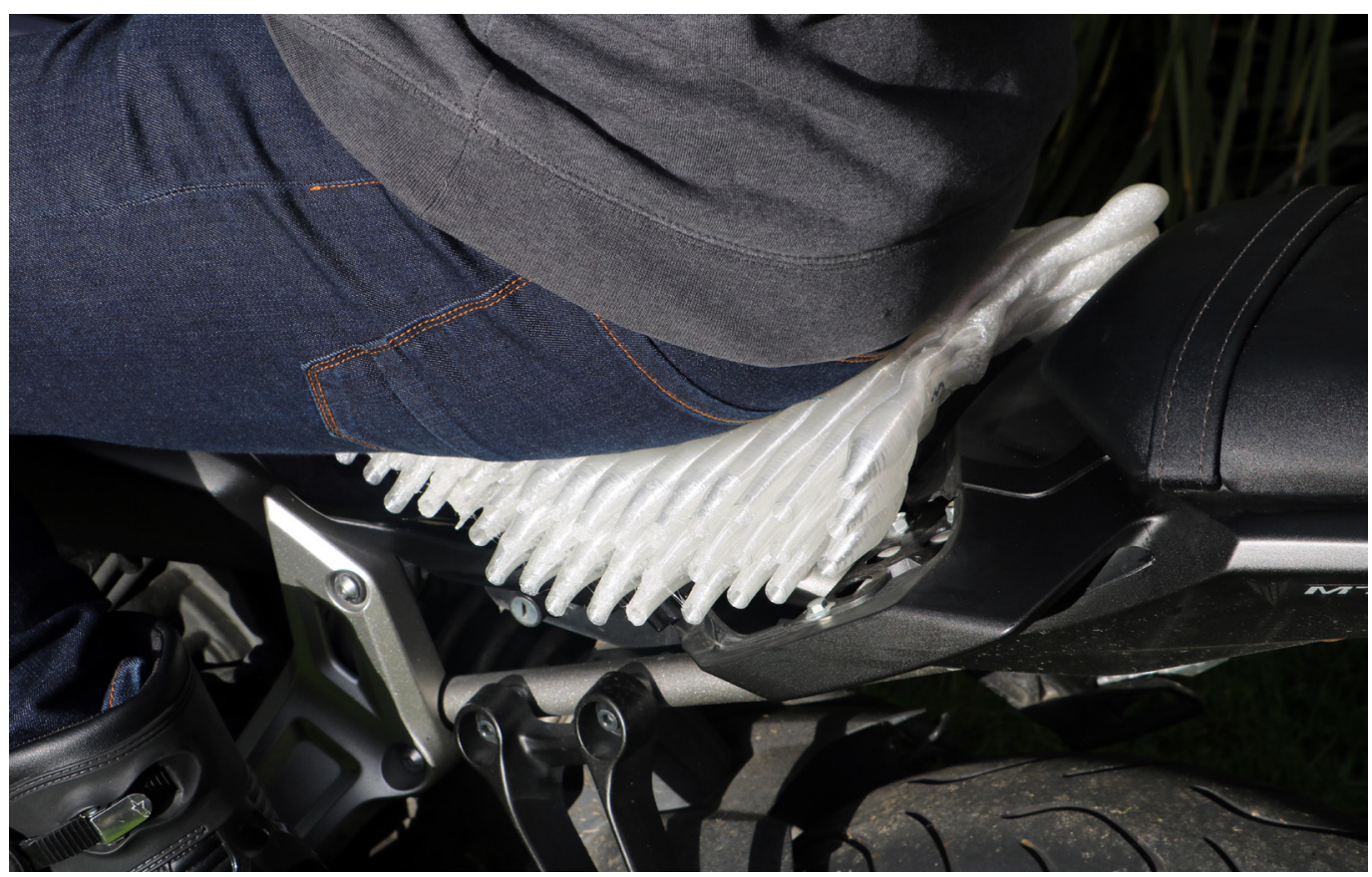




\section{Aesthetic Development}

The aesthetics of this seat are directly derived from the function. When testing the different methods of creating support using FDM 3D printing, tubular structures were designed to compress under load. These structures gave an appropriate level of support which could be modified and varied to fit the proportions of individual riders. This working support system has been replicated within the design of the seat. It created a very strong aesthetic which not only performed well but also looked different to traditional seating design.

This small set of experiments explore the aesthetics of the seat in renders. These models focus on the edges of the seats and how these interface with the lines of the motorcycle.

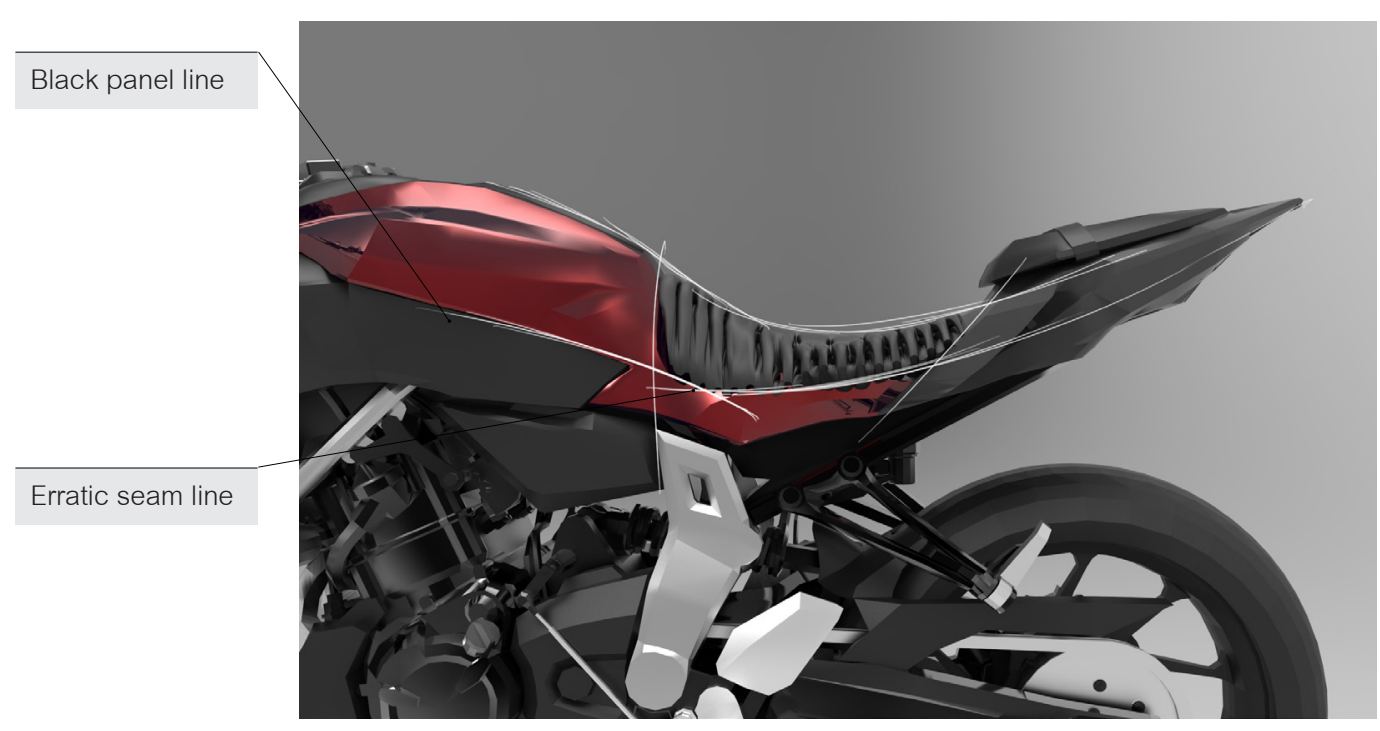

The ends of each tubular structure have been closed to form a single seam. This seam

follows the shape of the motorcycle, however, it has broken the motorcycle's smooth panel line.

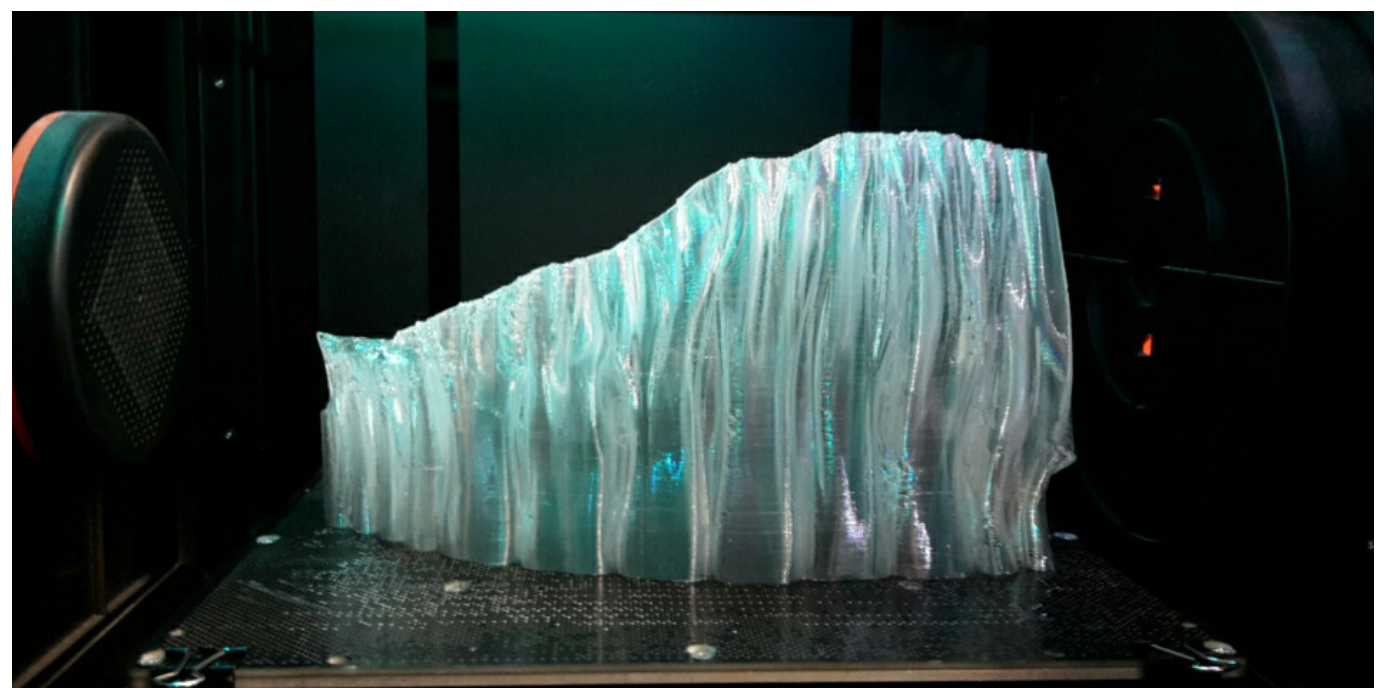

A high quality scale model printed in halves. 
This second scaled prototype introduces a geometric aesthetic. Due to machine error, this print is poor quality. The printer has printed the model at an increased temperature. This has caused the material to boil as it is being extruded filling it with small bubbles reducing the opacity.

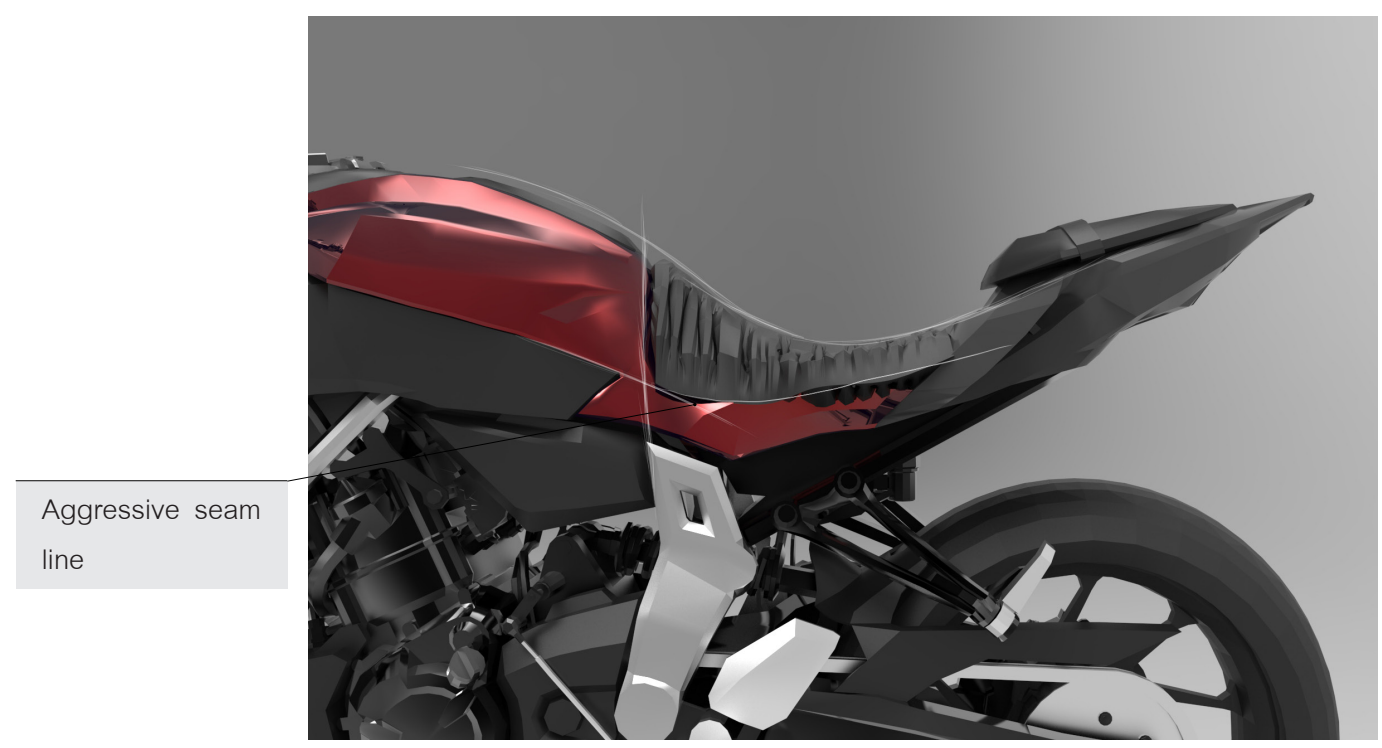

This prototype takes a more aggressive aesthetic using straight lines as opposed to curves.
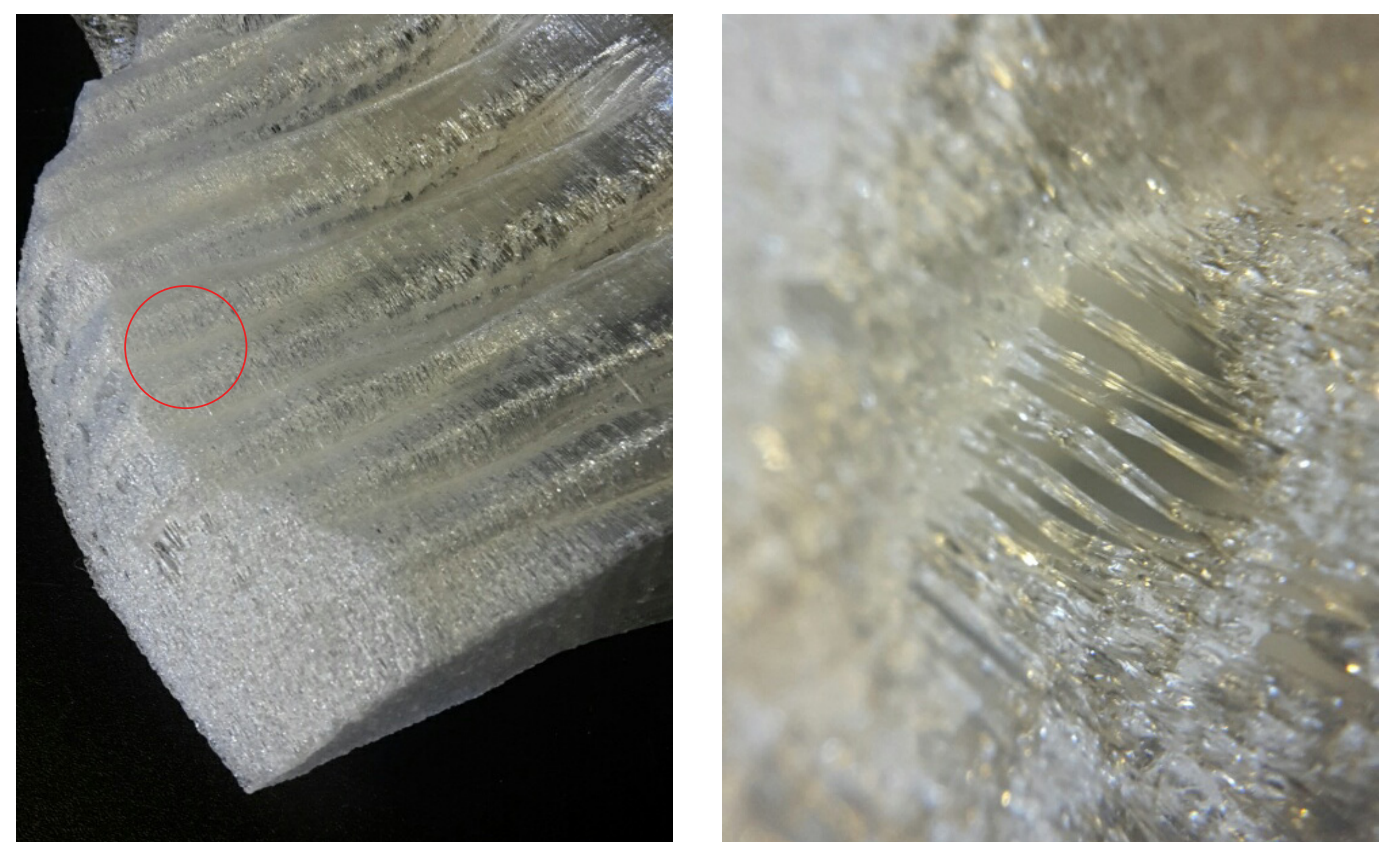

There is a clear difference between the quality of this scale prototype and the previous. 


\section{Aesthetic Development}

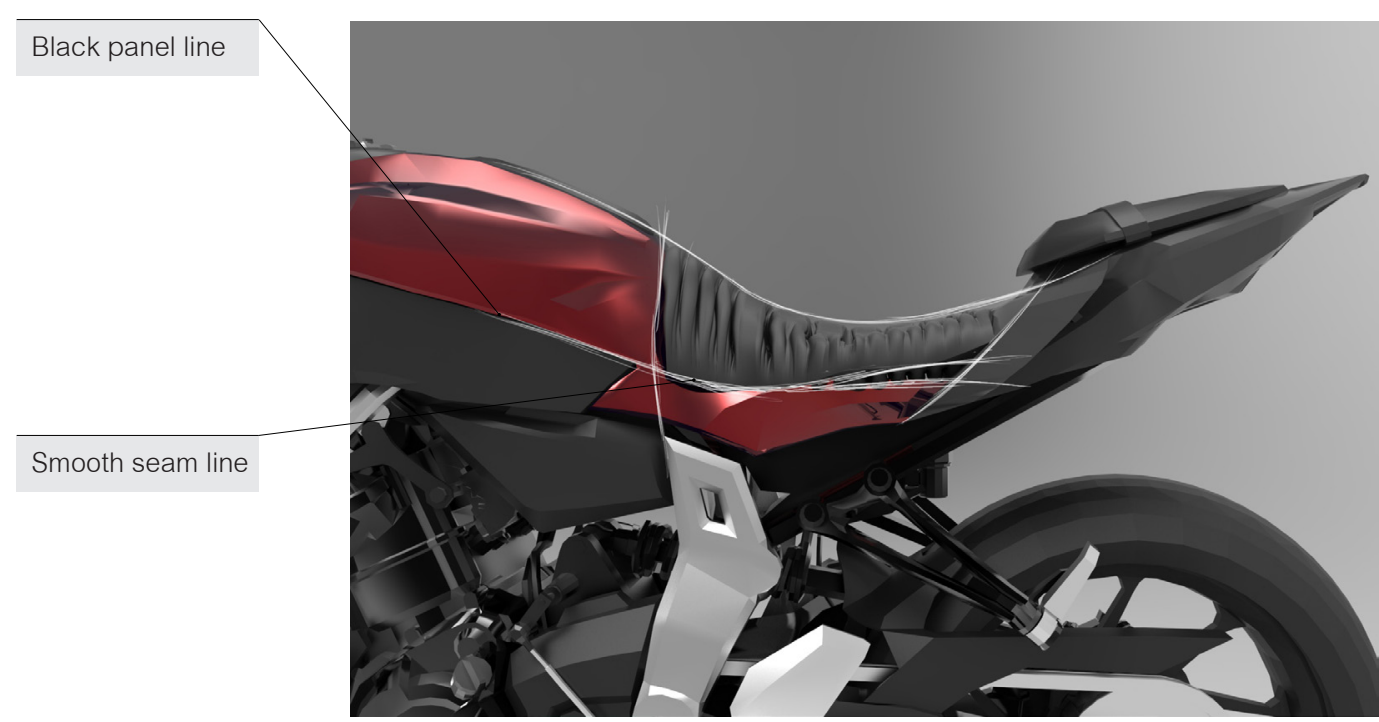

Figure 35 - Aesthetic seat prototype.

The seam at the edge of the seat in figure 35 has been refined. This seam flows from the black panel line down the length of the seat and up to the rear of the motorcycle. This creates a balanced interface between bike and seat. The problem with this design is it is difficult to 3D print as it does not conform to the draft angles earlier identified. Some areas of the print failed due to the excessive overhang of the shape. To resolve this the overhanging angles will be reduced giving each layer something to build on. The balance between aesthetics and manufacturing functionality is important and needs to be well considered within the design of the seat.
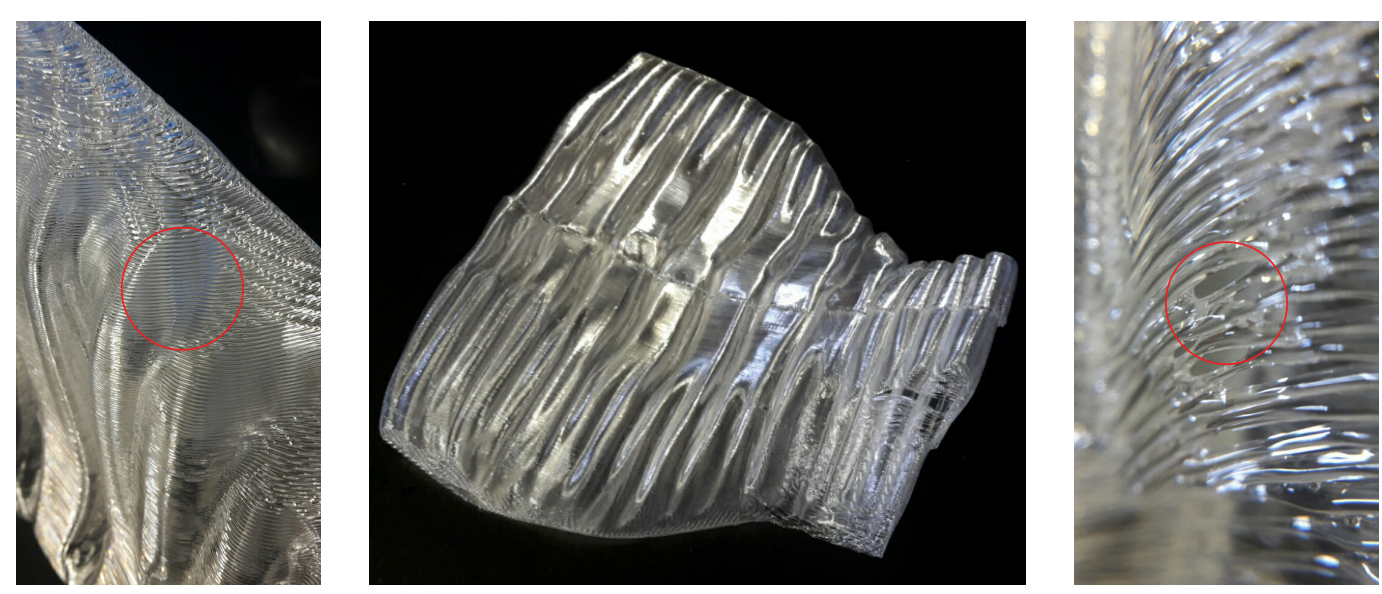

The above models illustrate the material qualities of this scale prototype. There are apparent patterns within the material that are similar to those explored during the small scale material experiments.

\section{REFLECTION:}

The aesthetic of the seat and the way it interfaces with the rider and motorcycle are important. The two lines on the motorcycle (the top of the black panel and the line moving from the rear seat down through the rider's seat) are the key components identified to allow the seat to successfully interface with its design. The tubular design of this seat is different to traditional upholstered seats. It creates an interesting aesthetic which shows off the capabilities of the technologies used throughout this research, as well as emphasising its functionality. The next set of full scale prototypes explores the individual problems with each prototype, making small iterations to refine the performance of each seat design. 


\section{Prototype 4, 5}

Previous results highlighted the requirement for a slightly softer seat. Prototype 4 and 5 focus on refining the performance of the structure of the seat to provide improved support and cushioning.

Prototype 4 uses a double layer wall thickness. It was quickly established that printing Prototype 4 as a single layer wall thickness made the seat too soft. Figure 36 demonstrates a single layer wall compared to a double layer wall when supporting a load. Consequently the tubes in the centre of the seat have been joined to lessen the overall support. However, when physically tested, the seat lacked density. This is reflected in Figure 37.

Add another set of wide tubing increasing the level of support.

Push these tubes together and add an extra tube at the end. 

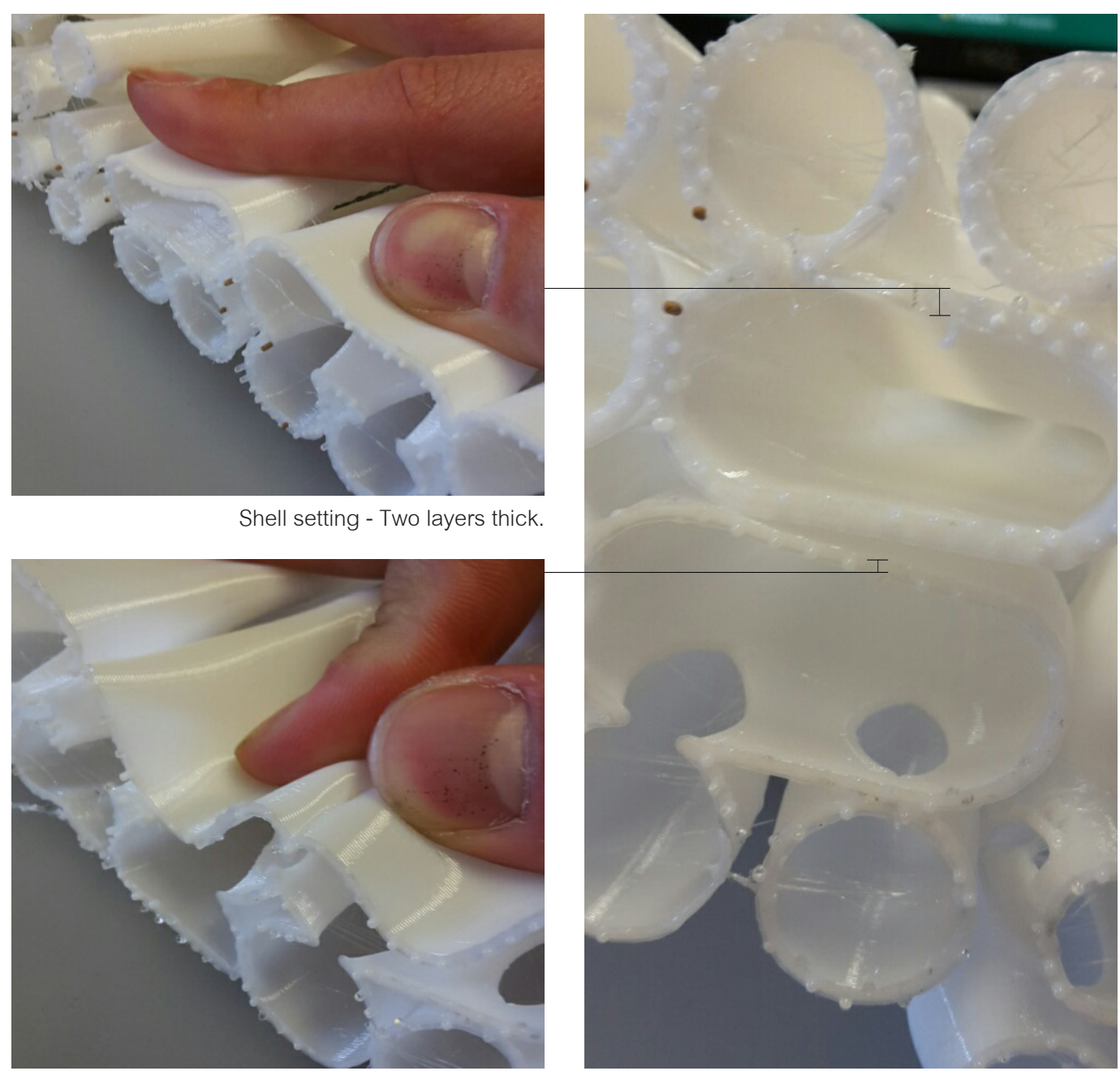

Shell setting - Two layers thick.

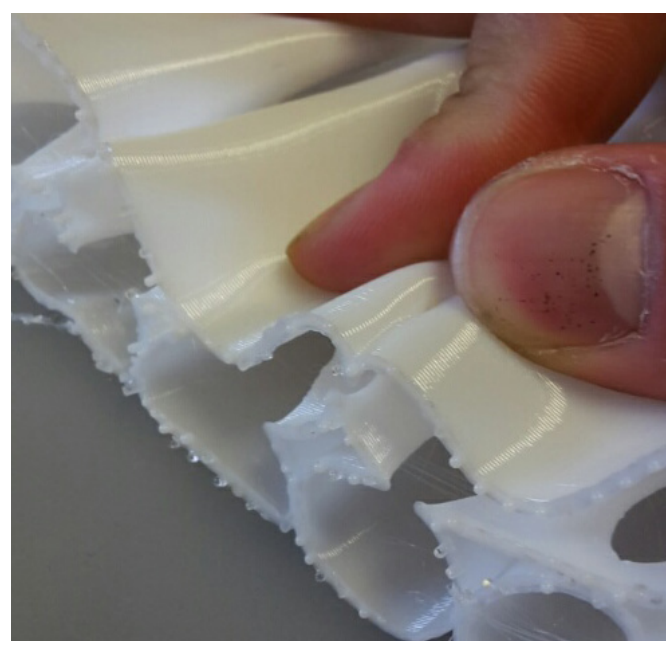

Surface setting - One layer thick.

Figure 36 - Thickness comparison

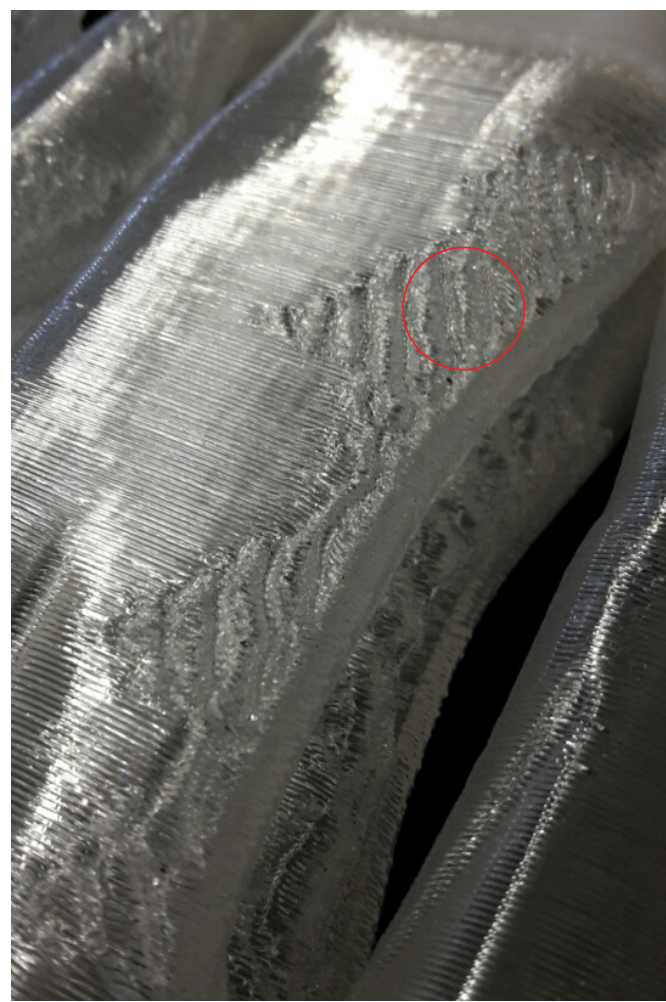

Textures being created onto the surface of the seat as the printer prints angled layers.

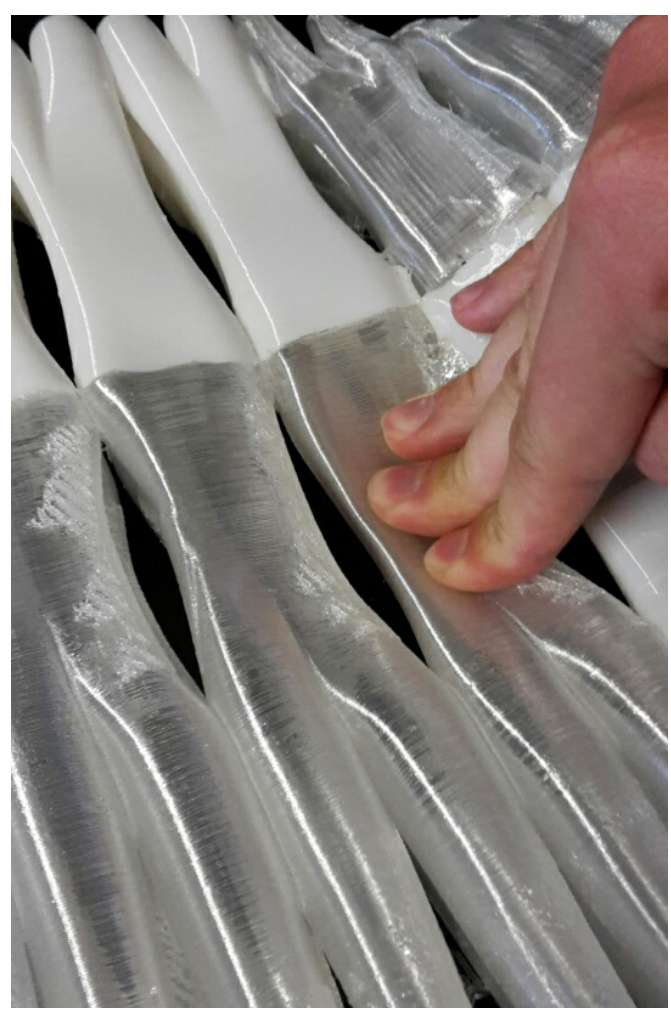

Compression testing finding the ideal amount of support. 


\section{Prototype 4, 5}

Prototype 5 expands on the lack of support experienced by the previous prototype, however, the way in which this seat is constructed is different to the previous ones. The seams that have been created between each piece are positioned directly on top of the area where the rider's ischial tuberosity bones are positioned (Figure 38). This is the area of greatest pressure, and these seams make it extremely uncomfortable to sit on.

\section{REFLECTION:}

Prototype 4 and 5 have revealed interesting structural qualities. It has been established that the combination of a double layered wall thickness with a form similar to Prototype 5 provides an appropriate level of support and comfort to the motorcycle rider. The next development of these full scale prototypes is to print them at full scale in a single print rather than as assembled parts. This transition will resolve the discomfort of sitting on hard seams.

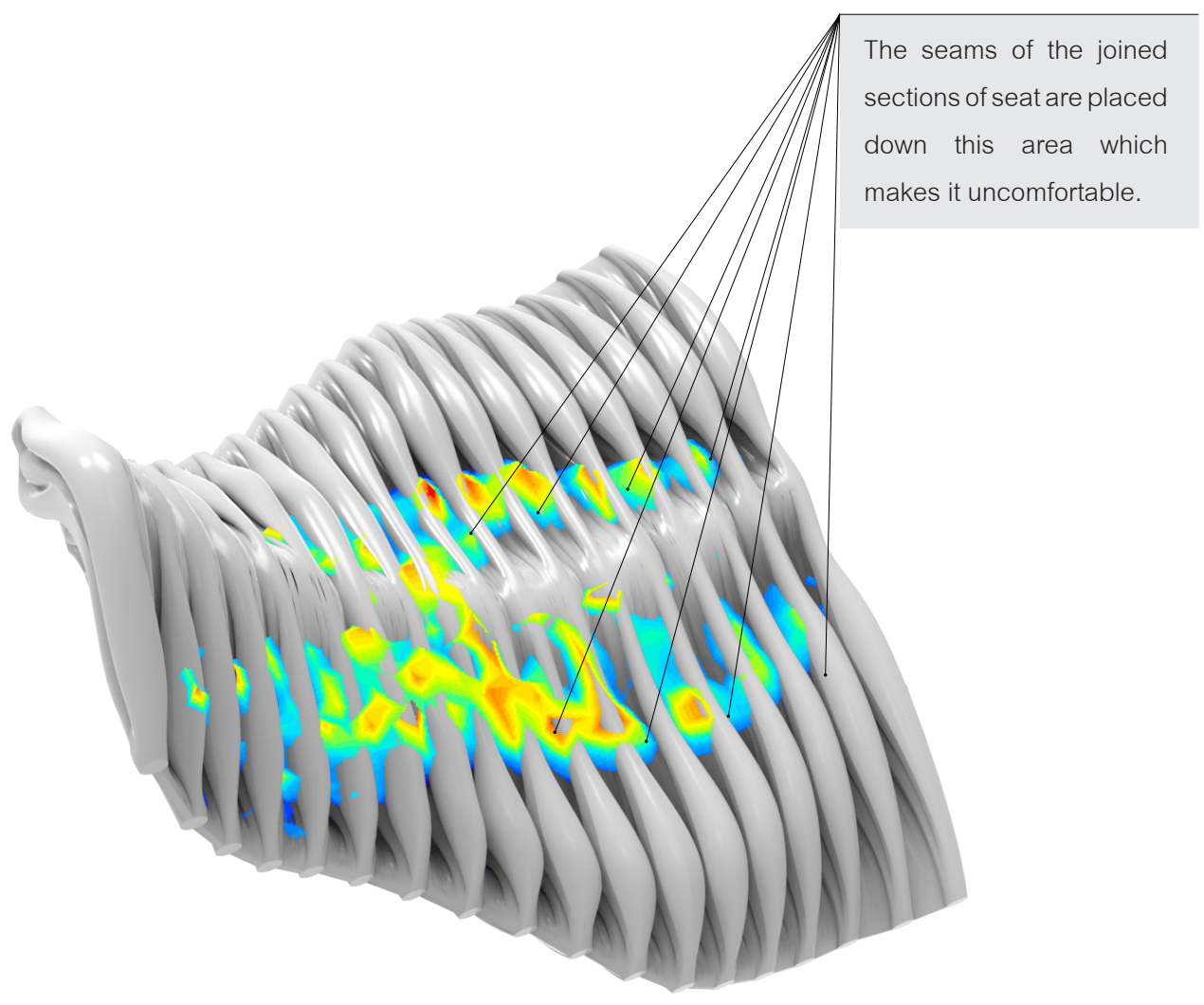

Figure 38 - Prototype 5 


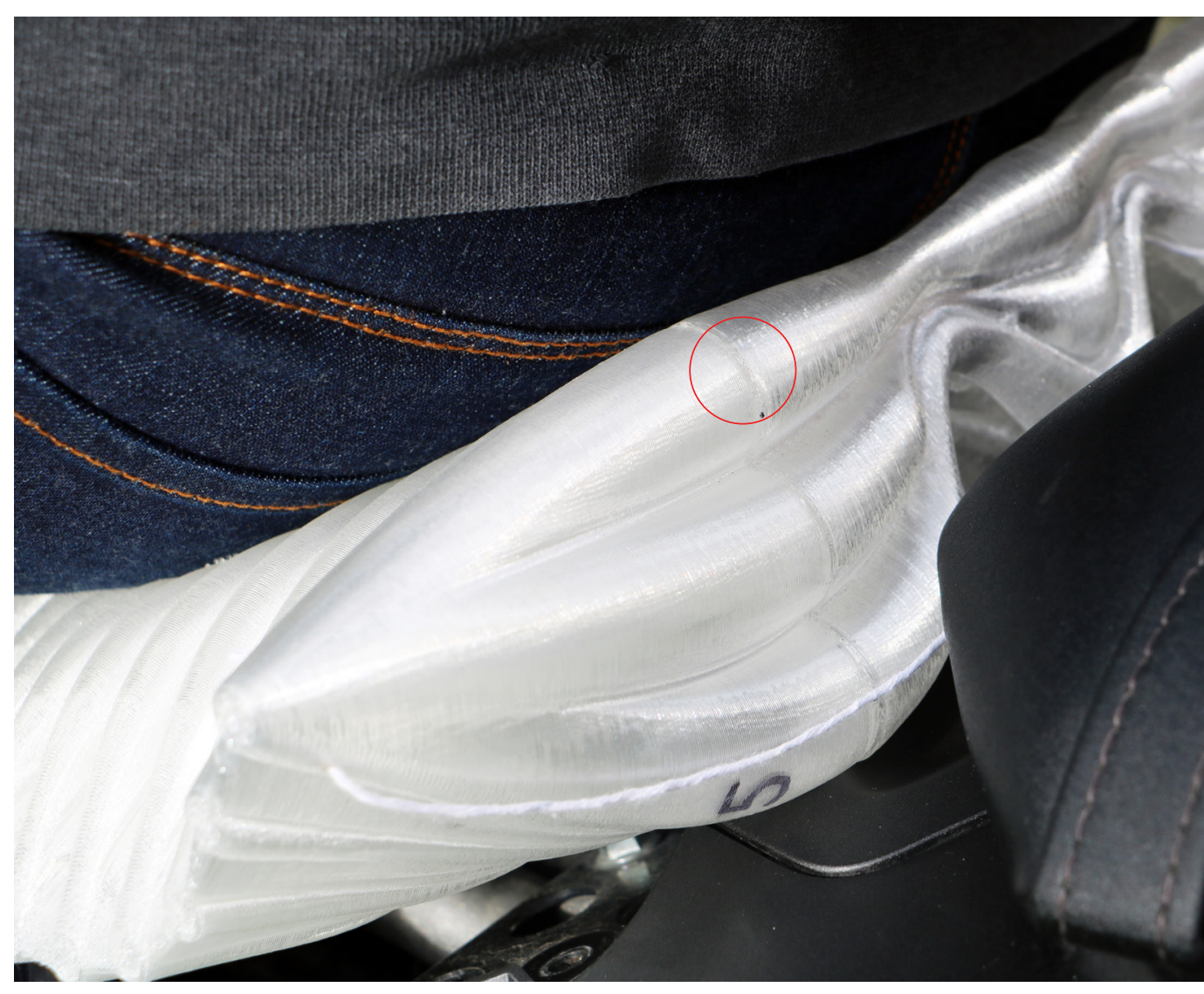

This is the seam where the multiple 3D printed parts have been joined.

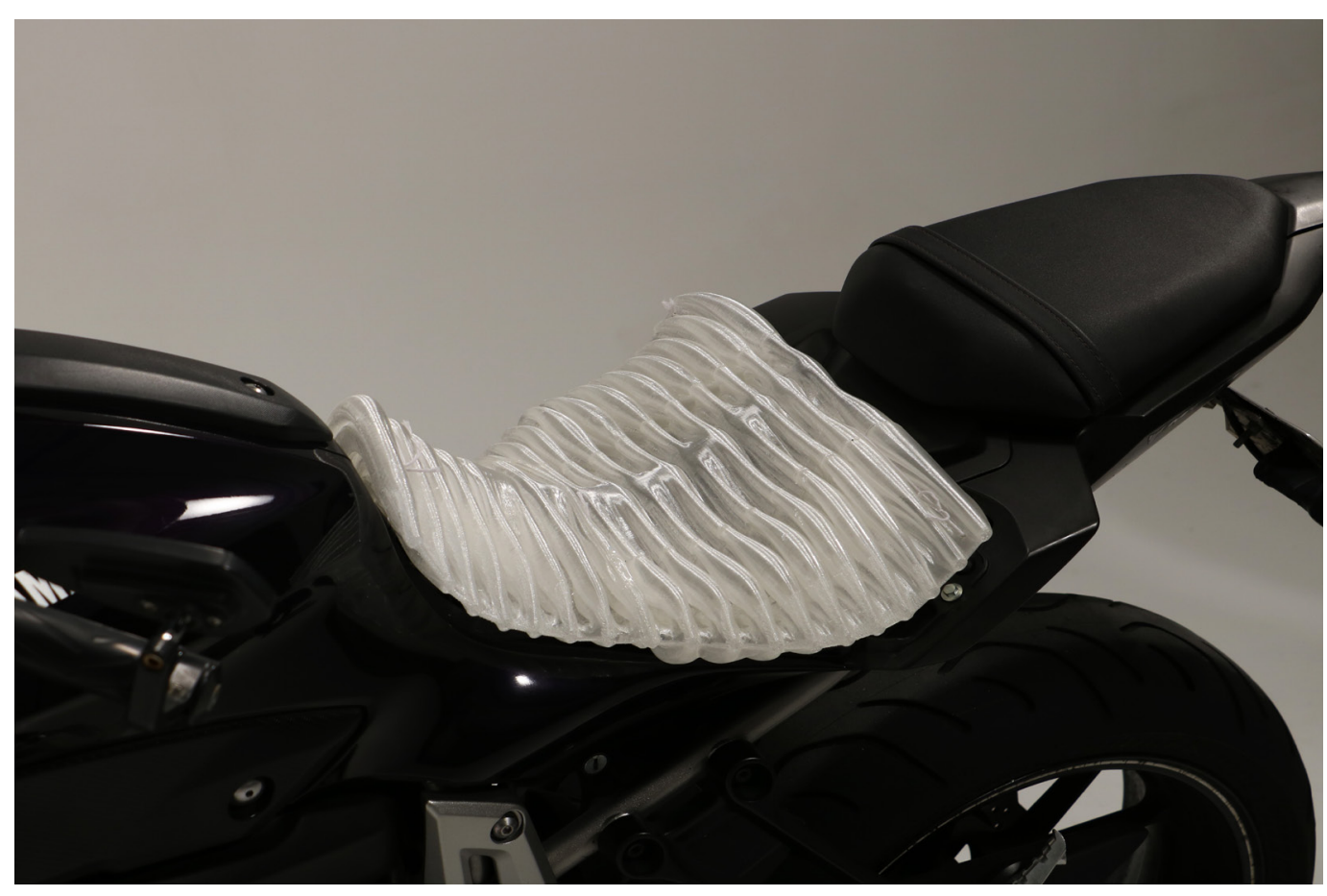




\section{Full Scale Testing}

To develop the prototypes to be 3D printed in a single print, a Big Rep 3D printer is used.

A series of low fidelity test prints are used to learn the processes of the Big Rep printer before building full-scale seat prototypes. Small sections of the full-scale seat are printed to learn how the raft, support structures and overhangs are dealt with at a larger scale.

The Big Rep printer has a $1 \mathrm{~mm}$ nozzle as opposed to the $0.8 \mathrm{~mm}$ nozzle previously used on the Up Box 3D printer. Consequently, this makes the tube structures that were previously printed as part of the seat, feel too hard (Figure $39 \& 40$ ). The material used for this latest testing is a Thermoplastic Elastomer (TPE) plastic. This is different to the TPU plastic which has been used at small scale, and although it is flexible, TPE it is harder and weaker than TPU.

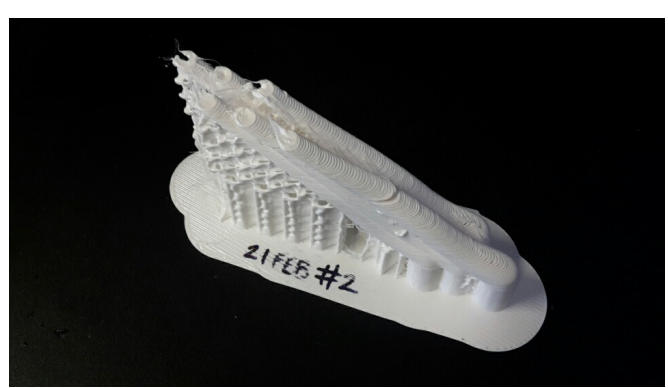

Figure 39 - Large scale test models.

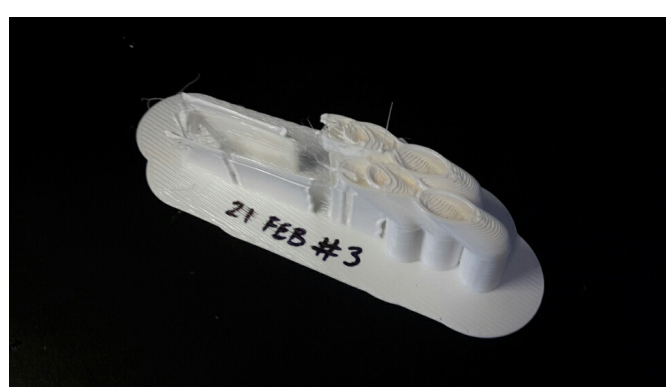

Figure 40 - Large scale test models.

Figure 41 shows a section of the full-scale print which failed half way through being built. This model had its own custom support CAD modelled into the object itself. However, the support was insufficient causing the edges to peel off the bed.

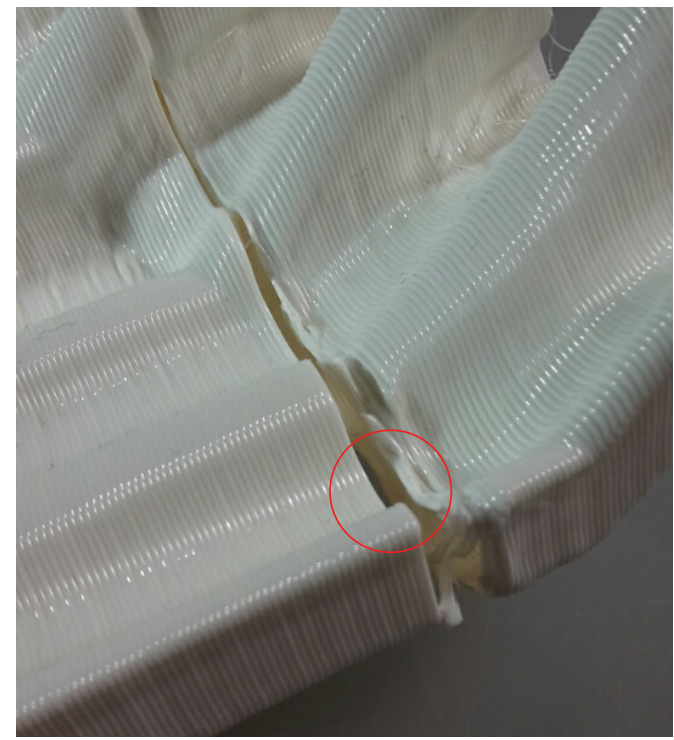

Figure 41 - Breakage in layer adhesion caused by the model moving on the bed of the printer.

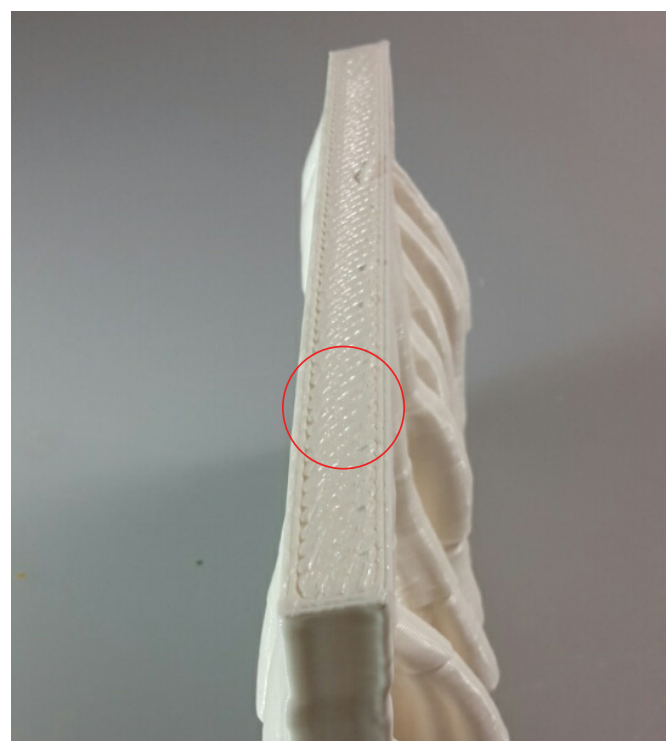

Narrow insufficient raft. 
To resolve the issue, an increased raft has been added (Figure 42). Although the raft helped, the model had to be weighted down mid-way through the print as it began to lift. As the object's build height increased the model became less stable.

The nozzle began wobbling the model side to side as it was printing, causing it to fail.

To resolve this problem, additional support is added to assist in adhering the object to the bed as well as increasing the model's print stability.

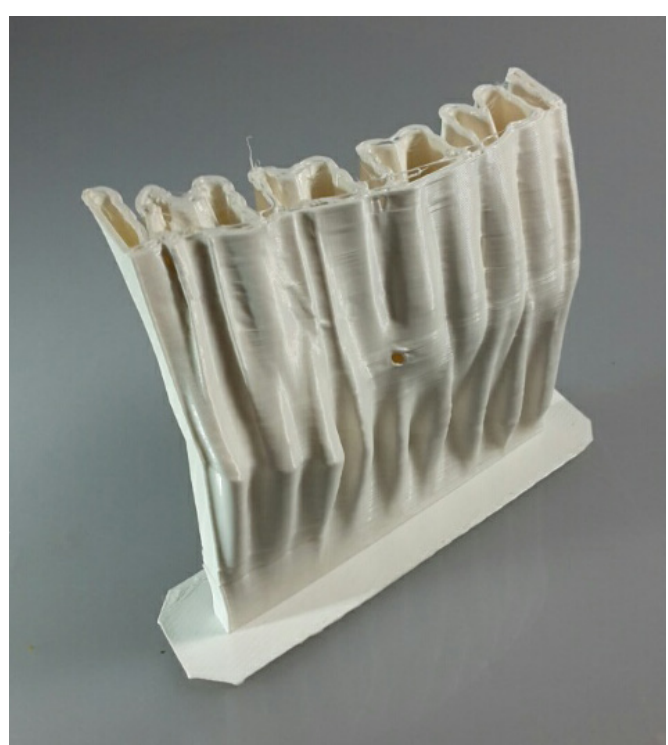

Figure 42 - Unsuccessful 3D printed model.

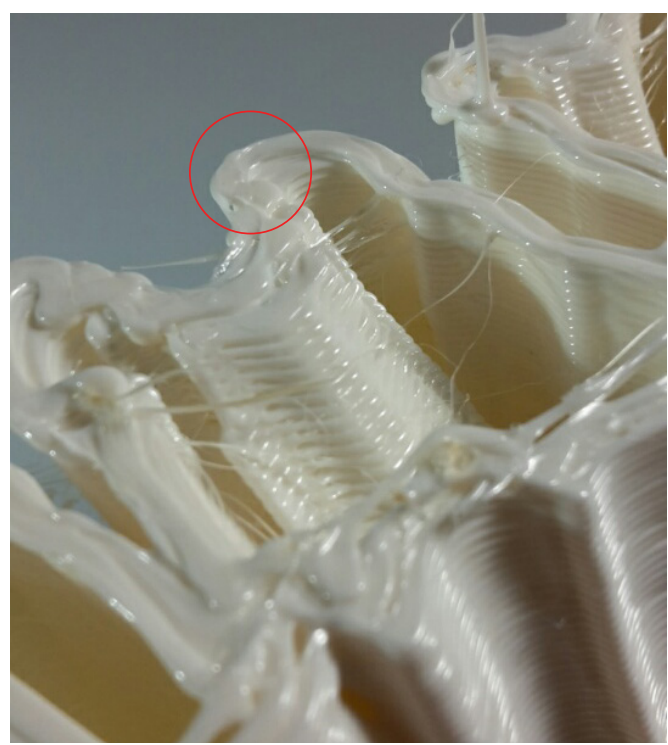

This build up of material is caused by the instability of the model. 


\section{Full Scale Testing}

A larger raft and support structure added to the bottom of the model produced a successful test. Printing as a single layered wall thickness using the Big Rep's $1 \mathrm{~mm}$ nozzle size gave an appropriate level of support and cushioning. This model was printed at full scale using TPU plastic.
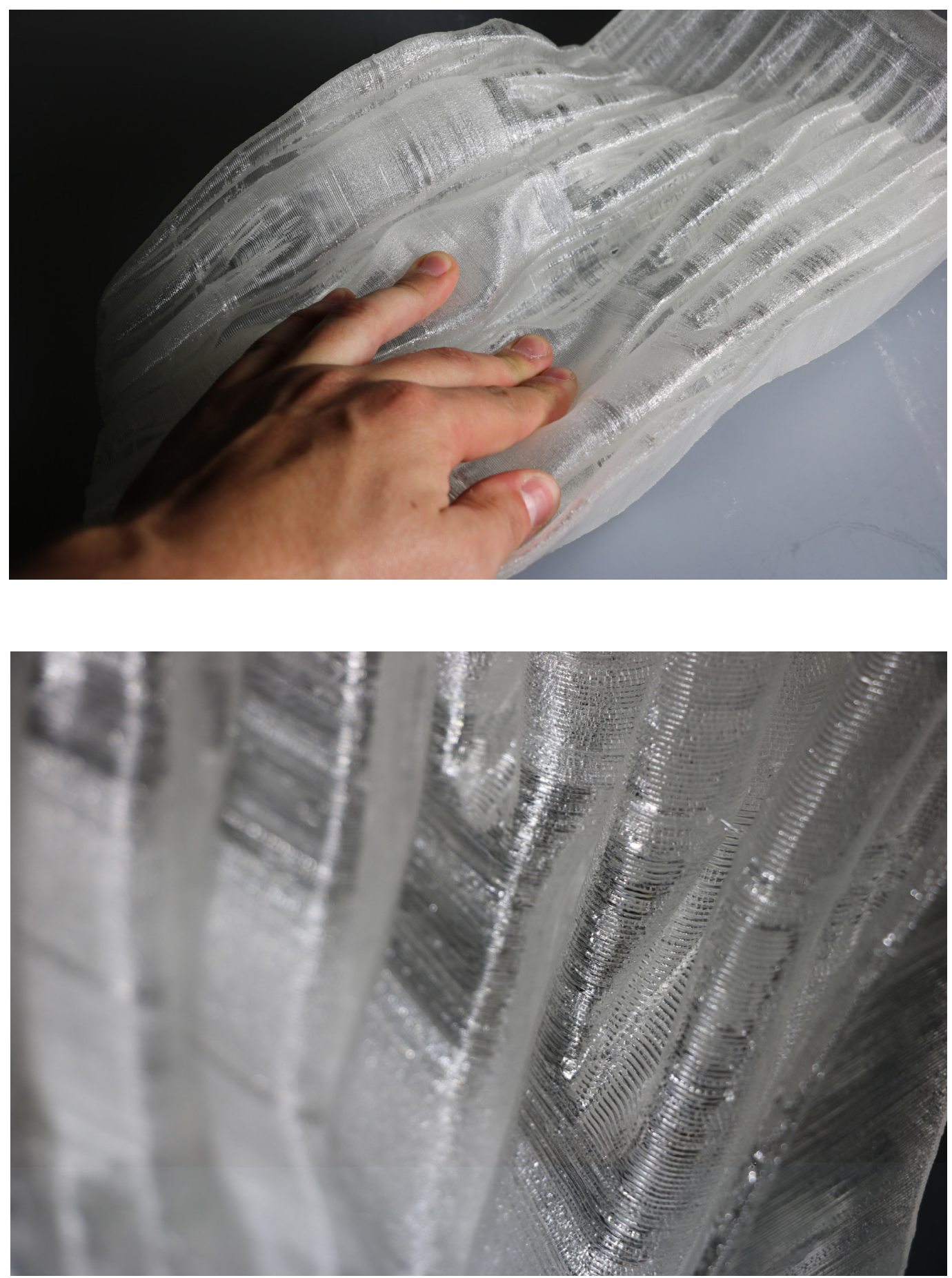

The above images illustrate the material qualities as well as how the structure compresses under load. 
This test has proven that printing a 1:1 scale motorcycle seat is viable. The balance between CAD model and machine have been refined allowing this custom seat to be successfully completed. Areas of the model have been identified where the draft angles are too great for the machine resulting in failed areas (Figure $43 \& 44$ ).

\section{REFLECTION:}

This series of full scale testing has allowed for successful 1:1 scale 3D printed seating to be built. It was previously discovered when creating the small scale models that printing at $200^{\circ} \mathrm{C}$ ensured the highest quality prints. This suggests that printing this seat with a lower temperature will provide a good quality and strong model. The overhanging areas will be adjusted within the model giving more area for the layers to build upon. These minor refinements in the model and machine temperature settings will allow for higher quality models to be executed ensuring quality and efficiency within the printing process.

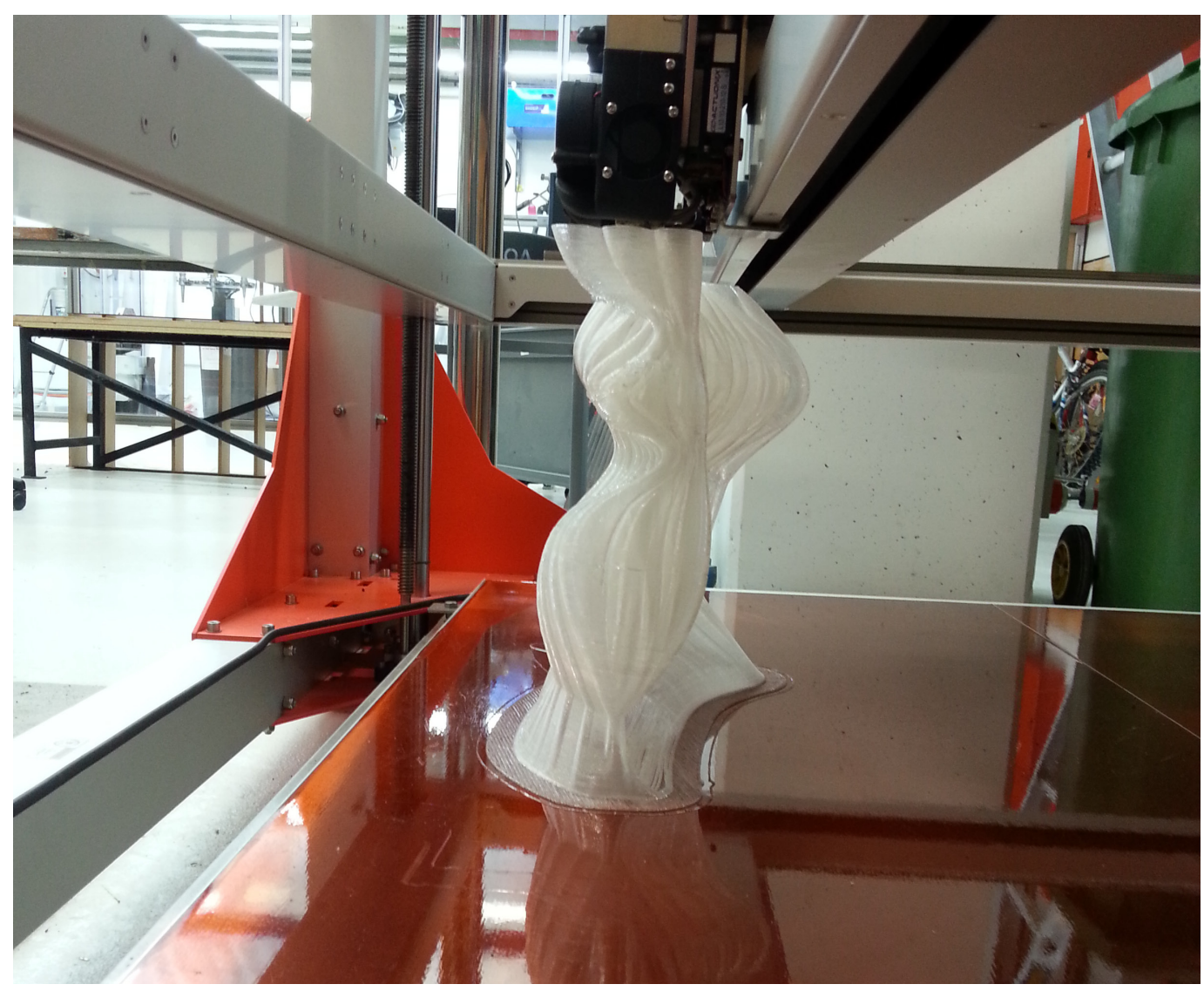

Full scale seat prototype being printed on the Big Rep 3D printer.

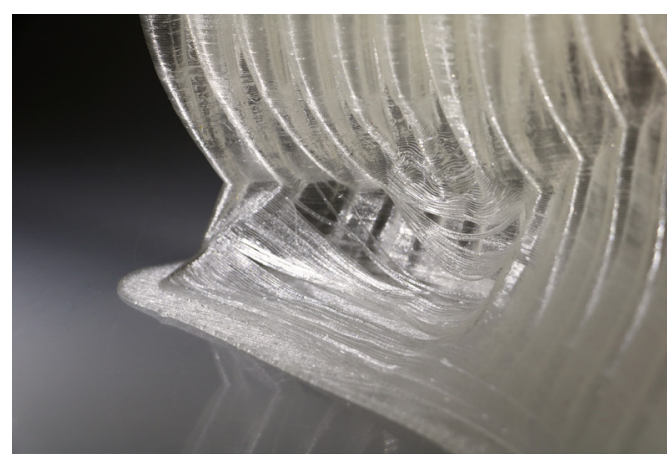

Figure 43 - Unsupported overhanging material.

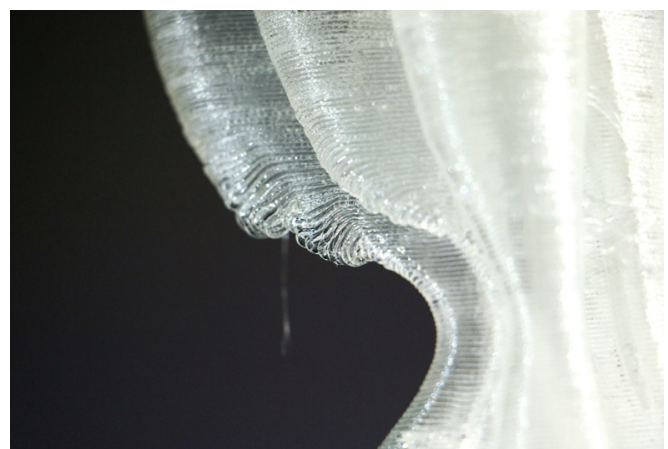

Figure 44 - Unsupported overhanging material. 
Prototype 6 and 7 explore small iterative refinements. These refinements allow for a welldeveloped final prototype where decisions have been carefully considered.

Prototype 6 has been designed with a sacrificial raft used to create more surface area to adhere to the printer bed. Through pressure mat testing, this prototype has revealed an ideal level of support to the rider. Figure 45 illustrates how the seat provides an even spread of support to the form of the rider. Although the peaks of the pressure map are slightly higher when compared to other prototypes, from the rider's opinion this is the most comfortable prototype.

This prototype was printed at the same temperature as the previous. Reducing the temperature to $200^{\circ} \mathrm{C}$ will ensure a high quality successful print. The next iteration of the seat will also be shaped to fit the available seat space on the motorcycle.

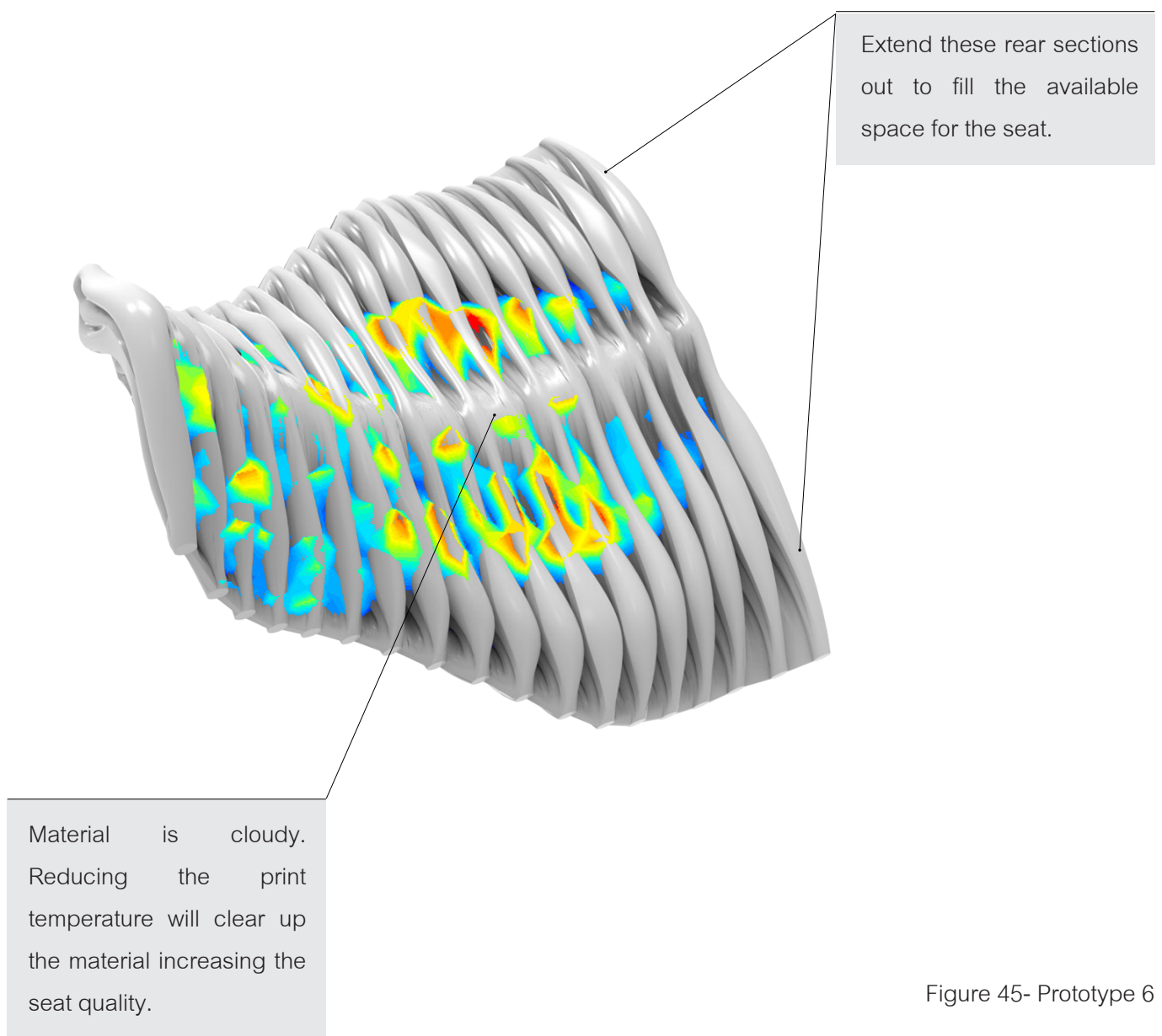



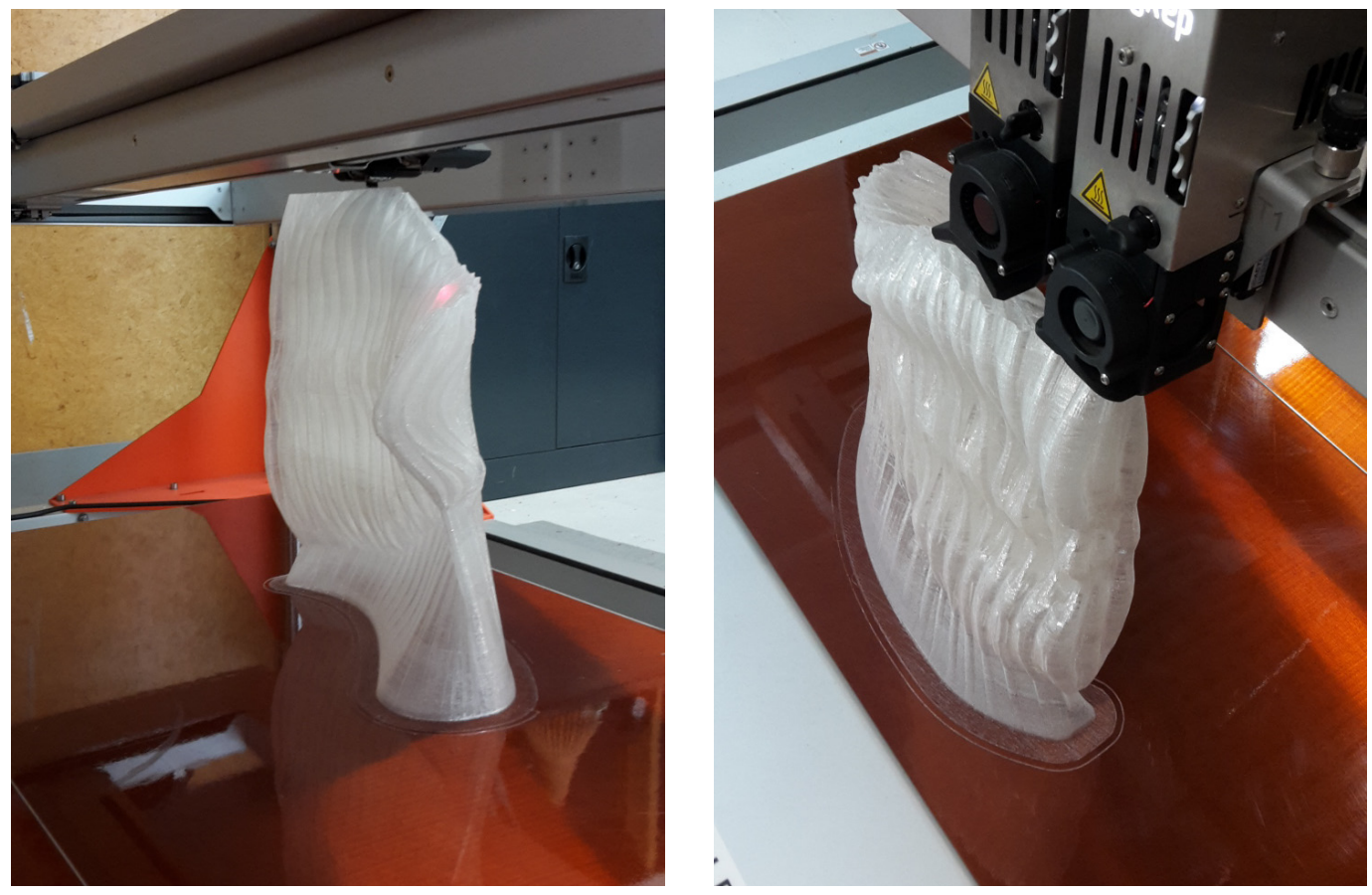

3D printing Prototype 6.

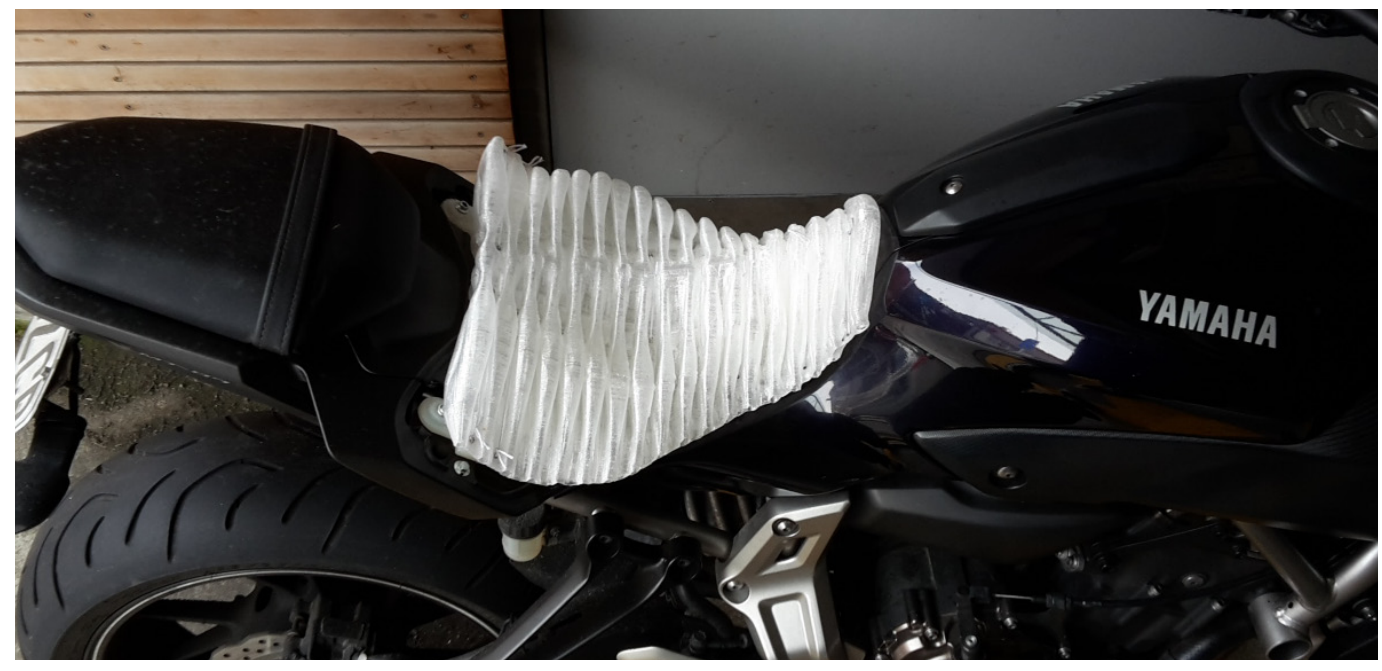

User testing Prototype 6.
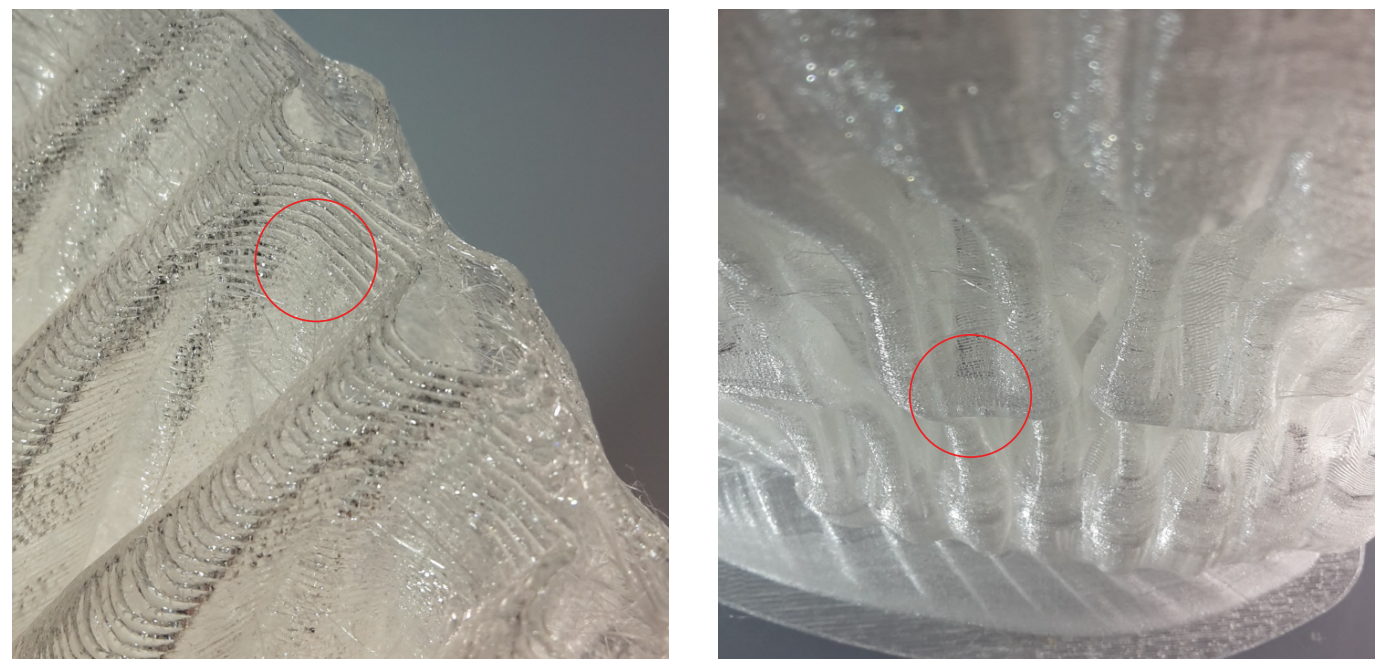

These images show the poor quality in material. The plastic should be crystal clear but it is cloudy. This is due to it being $3 \mathrm{D}$ printed at $210^{\circ} \mathrm{C}$. When reduced to $200^{\circ} \mathrm{C}$ it prints at a much higher quality. 
Prototype 7 is based on the same form as Prototype 6 but has been printed at $200{ }^{\circ} \mathrm{C}$ rather than $210^{\circ} \mathrm{C}$, improving the material quality. As well as a change in print temperature, the rear of the seat has been redesigned to aesthetically match the motorcycle.

This prototype was bolted to the hard PLA base and then ridden over the period of a week. At the start of that week the seat was not as comfortable as some of the earlier prototypes. However over the course of the week, the seat wore into the rider, similarly to a pair of shoes. The seat provided the rider with a comfortable level of support, evenly spreading their load over the surface of the seat (Figure 46).

\section{REFLECTION:}

Prototype 6 and 7 have assessed the usability of the full scale seats. These prototypes have been evaluated and refined to a level where the seat is not only comfortable to ride but also fits the shape and aesthetic of the motorcycle. To achieve a custom motorcycle seat that can be 3D printed, post processed, and bolted directly to the motorcycle requires a dual-material aspect. Where one part of the prototype provides the structure of the seat (PLA Component), the other provides support to the rider (TPU component). The next set of experiments will focus on dualmaterial 3D printing at large scale.

This seat provides a good level of support to the rider. The next step is multi-material $3 \mathrm{D}$ printing the base and padded support within a single print. 

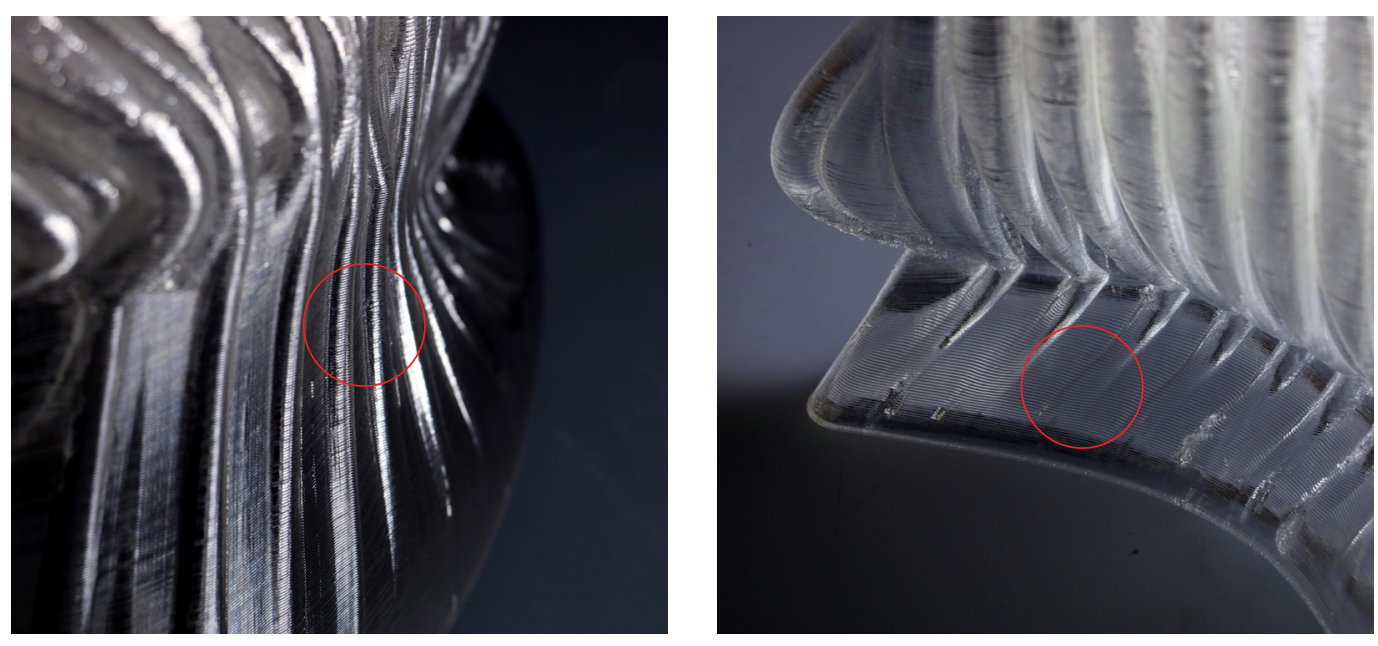

High quality TPU material printed at $200^{\circ} \mathrm{C}$.

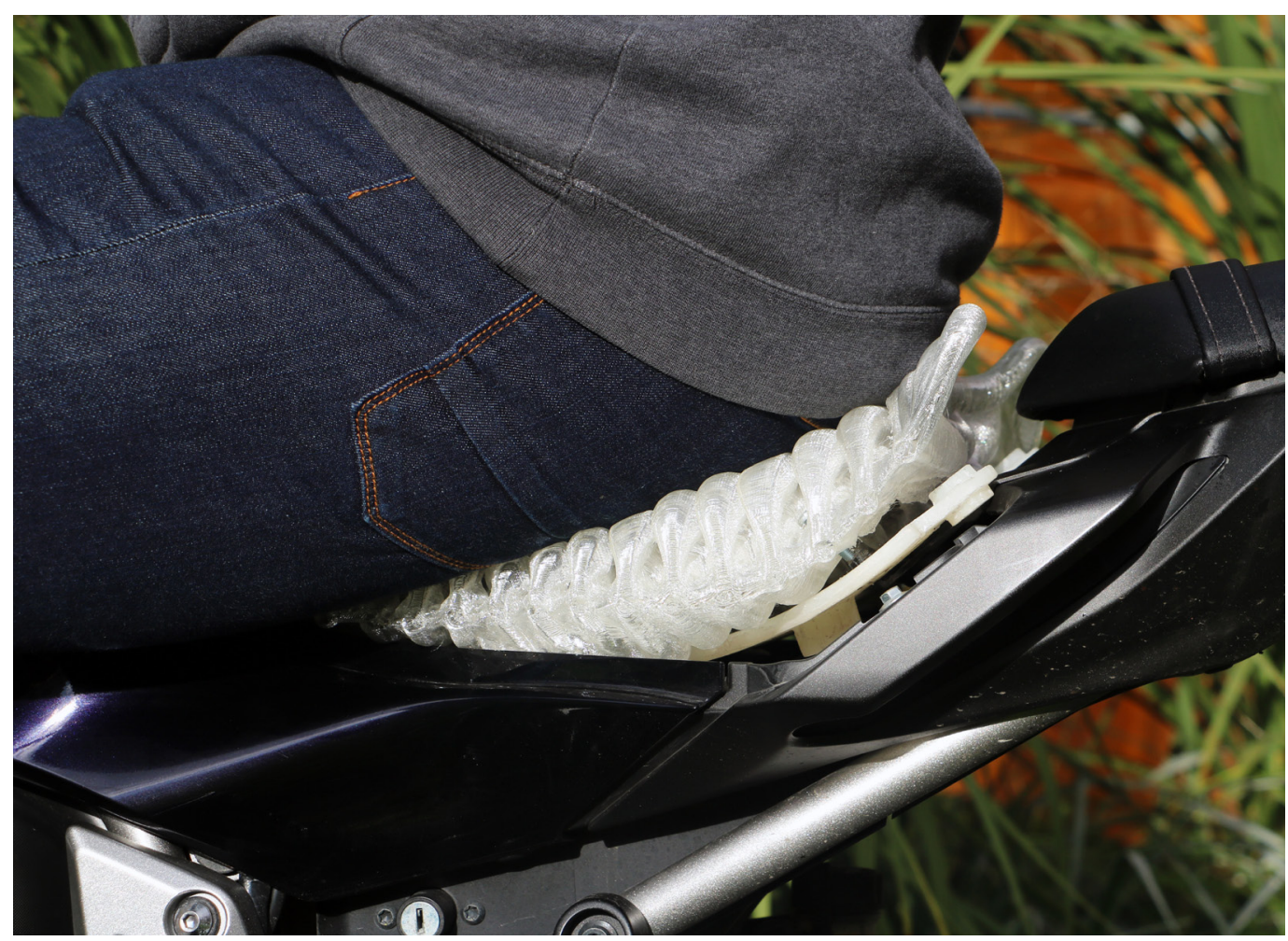

Bolts were used to mount the support component of the seat to the base of the seat. This ensured the seat could be safety secured to the frame of the motorcycle when ridden.

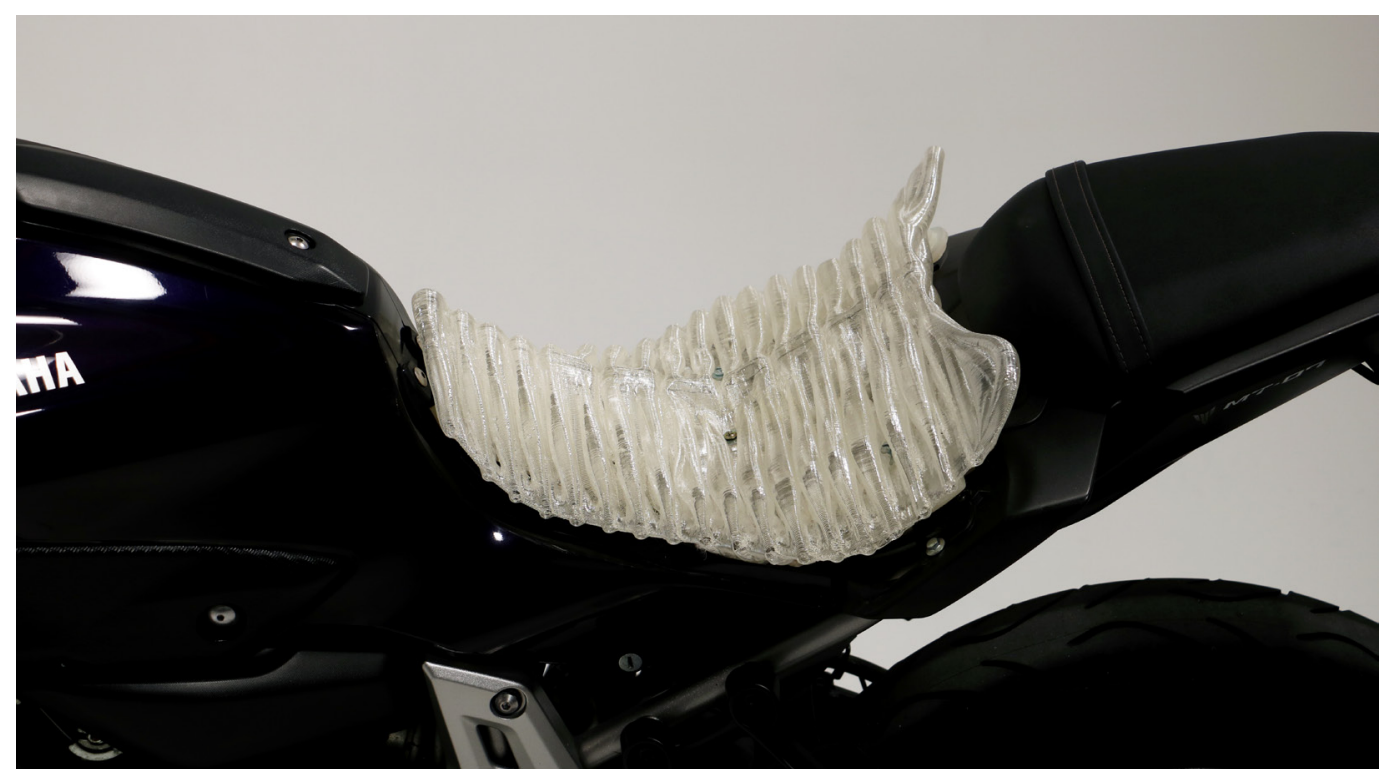




\section{Large Scale Dual-Material Testing}

Figure 47 is a small section of the full scale prototype. This test was printed in white TPU and clear TPU. This combination of materials has been chosen for this initial dual-material test to ensure that if the nozzle of the printer hit the model, then the TPU would flex and move out of the way, ensuring the printer wouldn't be broken. During this test, the movement speed of the printer was set too high compromising the material adhesion.

The model in Figure 48 is the same as the previous one. The speed of this print was reduced to half the speed which created a successful print. One problem which arouse from this test was that as one nozzle was printing, the unused nozzle oozed a $10 \mathrm{~mm}$ section of unwanted, additional plastic which could have been deposited onto the printed model. This caused some concern in regards to what would happen if the nozzle hit one of these pieces of material. However, this model has still been successfully printed even containing these imperfections.

The model was reprinted in hard PLA and soft TPU plastic (Figure 49). Once again this model was successfully printed although the unused nozzle deposited the same unwanted pieces of material.

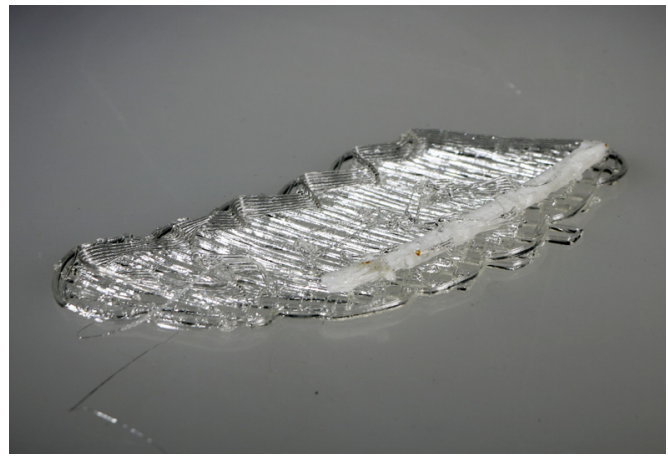

Figure 47 - Multi-Material FDM 3D printed test

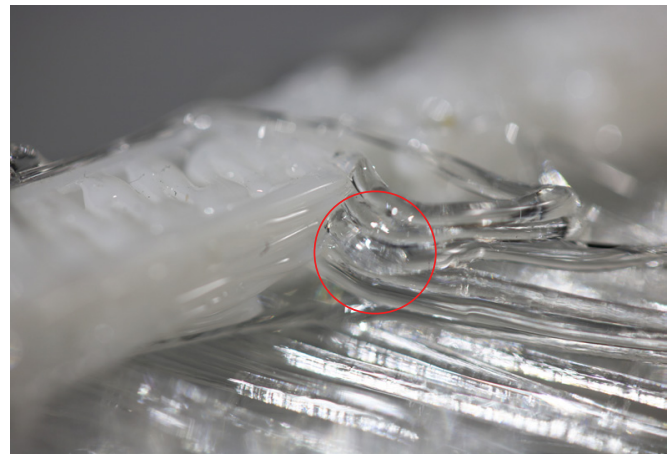

An example of the TPU material when it is printed at

$200^{\circ} \mathrm{C}$.

\section{REFLECTION:}

Slowing the machine's print speed has allowed for two consecutive, successful, dual-material 3D prints. However it is still concerning to see the small deposits of material wiped off the unused nozzle and onto the printed model. These deposits of plastic have the potential to escalate and cause the entire print to fail. This may be resolved by retracting the unused filament further inside the nozzle.

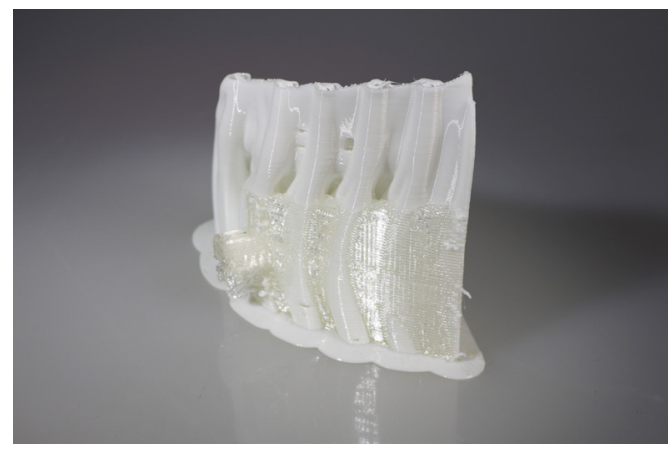

Figure 48 - Multi-Material FDM 3D printed test.

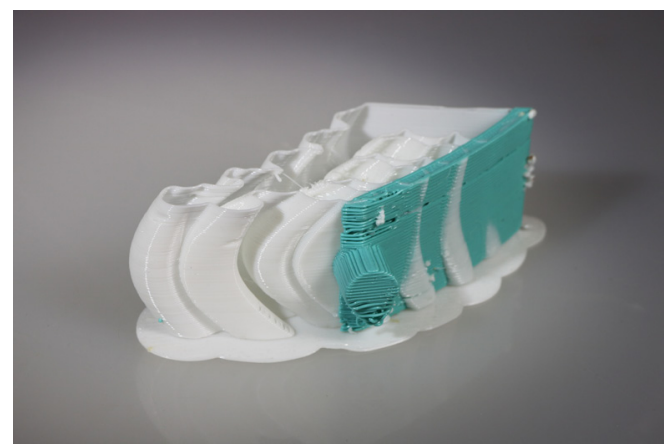

Figure 49 - Multi-Material FDM 3D printed test. 


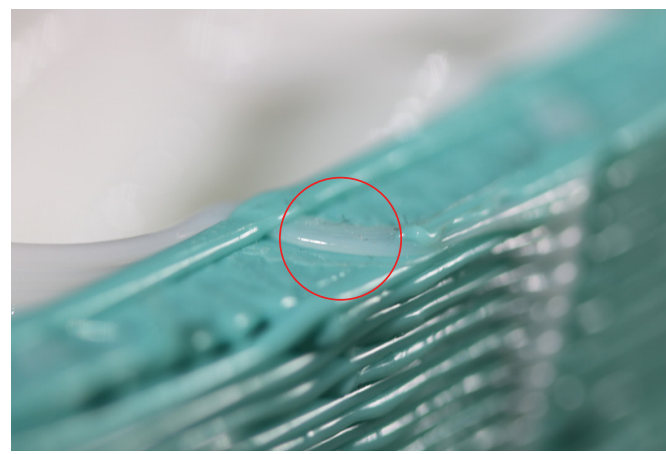

TPU material crosses a wall of PLA plastic.

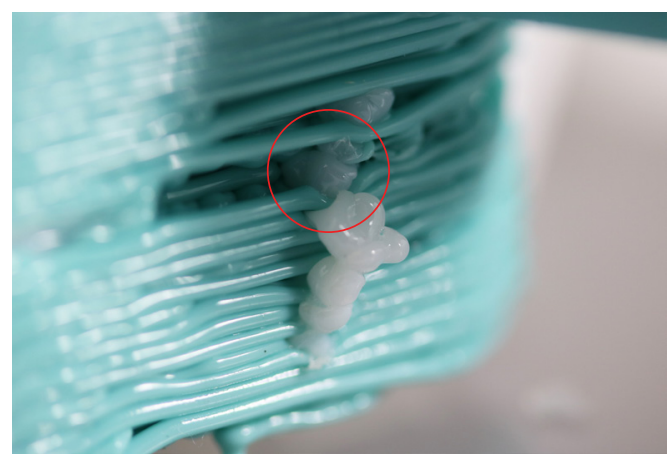

Unwanted deposits of TPU material within a wall of PLA plastic.
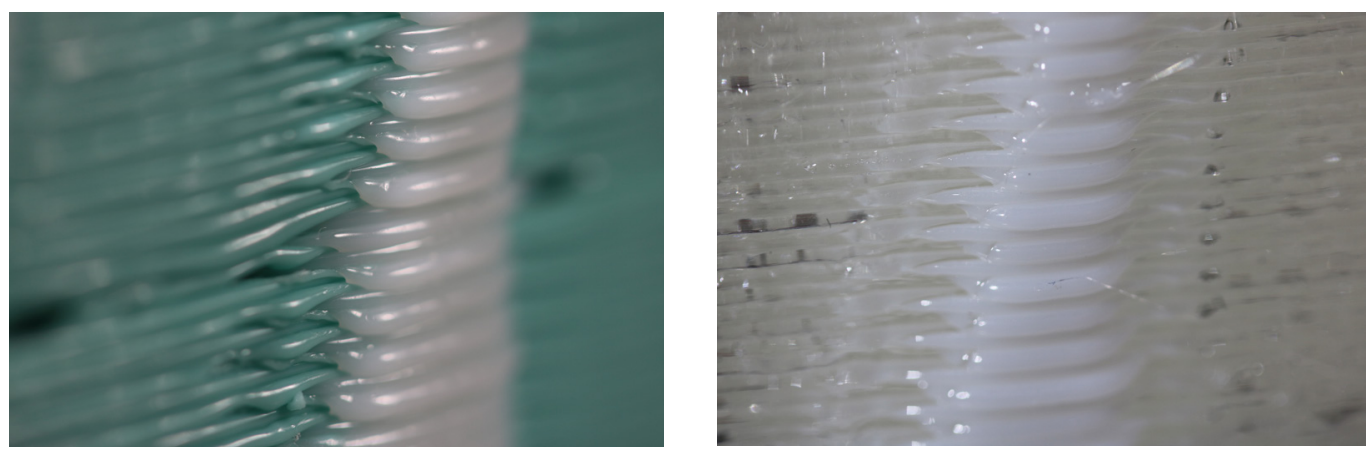

TPU and PLA materials have been incorporated into a single dual-material print. There is overlapping of materials as well as layers of TPU extruding through walls of PLA.

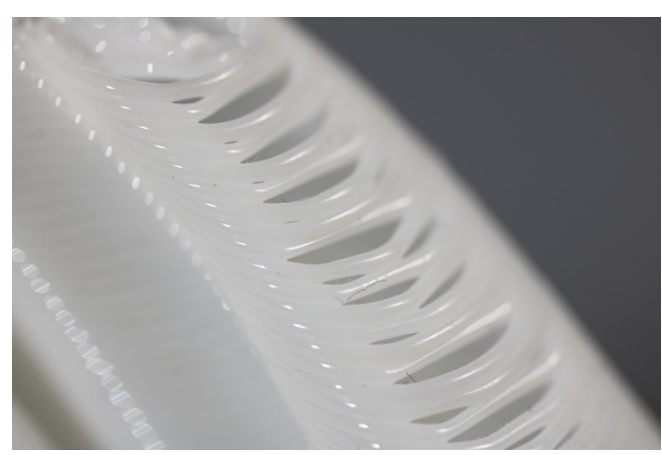

Pattern created on an angled part of the model.

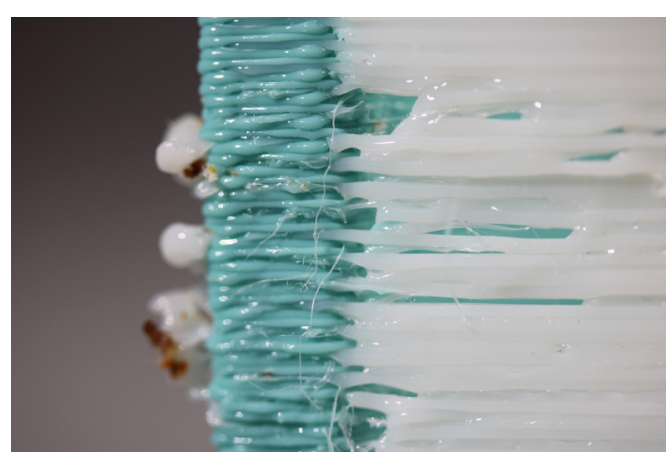

The poor material quality is caused by the change in nozzle heads as the object is being printed. 


\section{Prototype 8}

Prototype 8 was a full-scale dual-material motorcycle seat that took fifty hours to print. Some areas are poor quality, but overall the material is clear and strong. The hard and soft areas of the print are overlapping, creating a strong bond between the two materials. Figure 50 illustrates an area where extra deposits of material have been left in the model. Most of these can, however, be cleaned up during post processing.

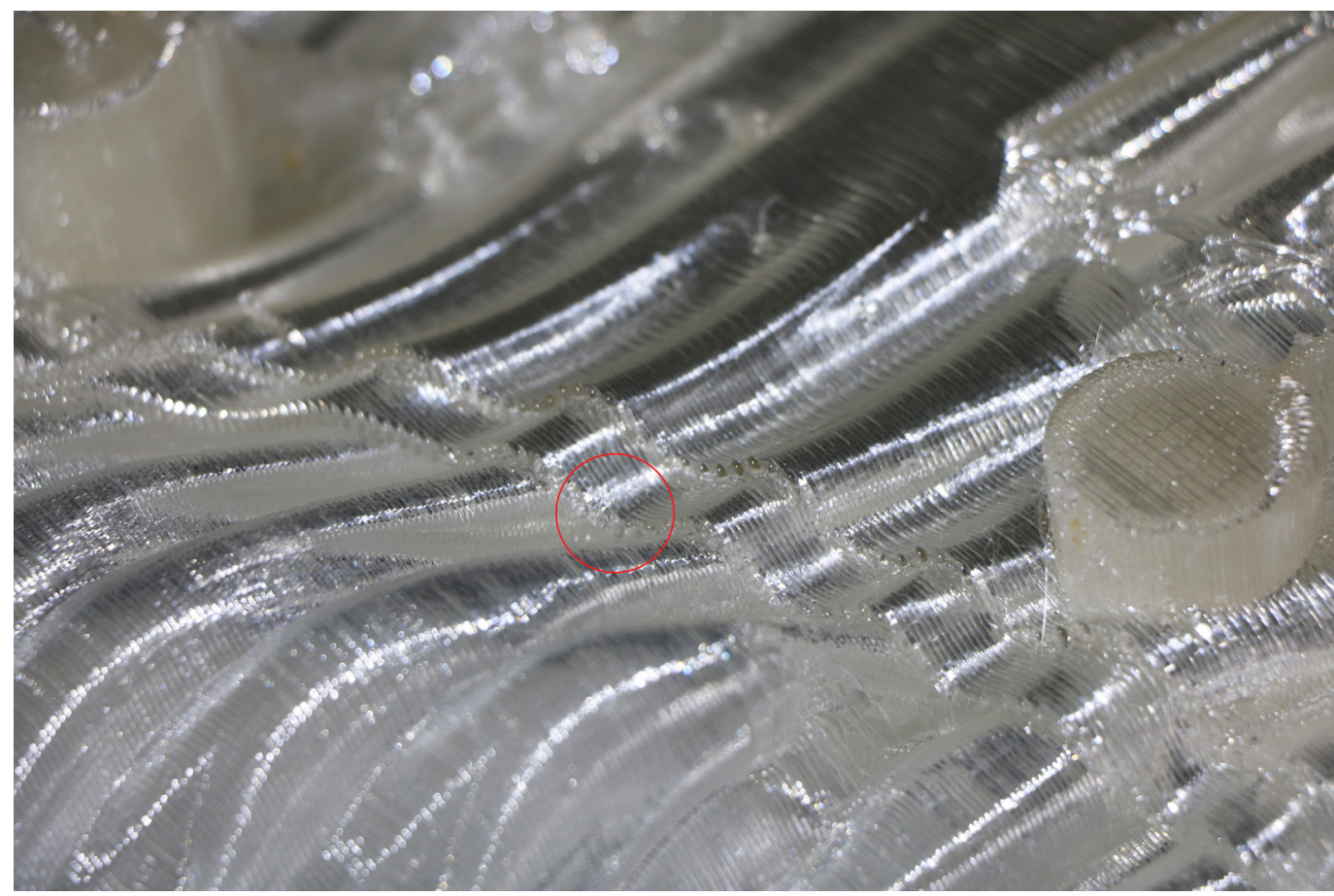

Edge where the PLA clear, and TPU clear materials meet. There is a strong bond between both these materials as they are adhered together during printing.

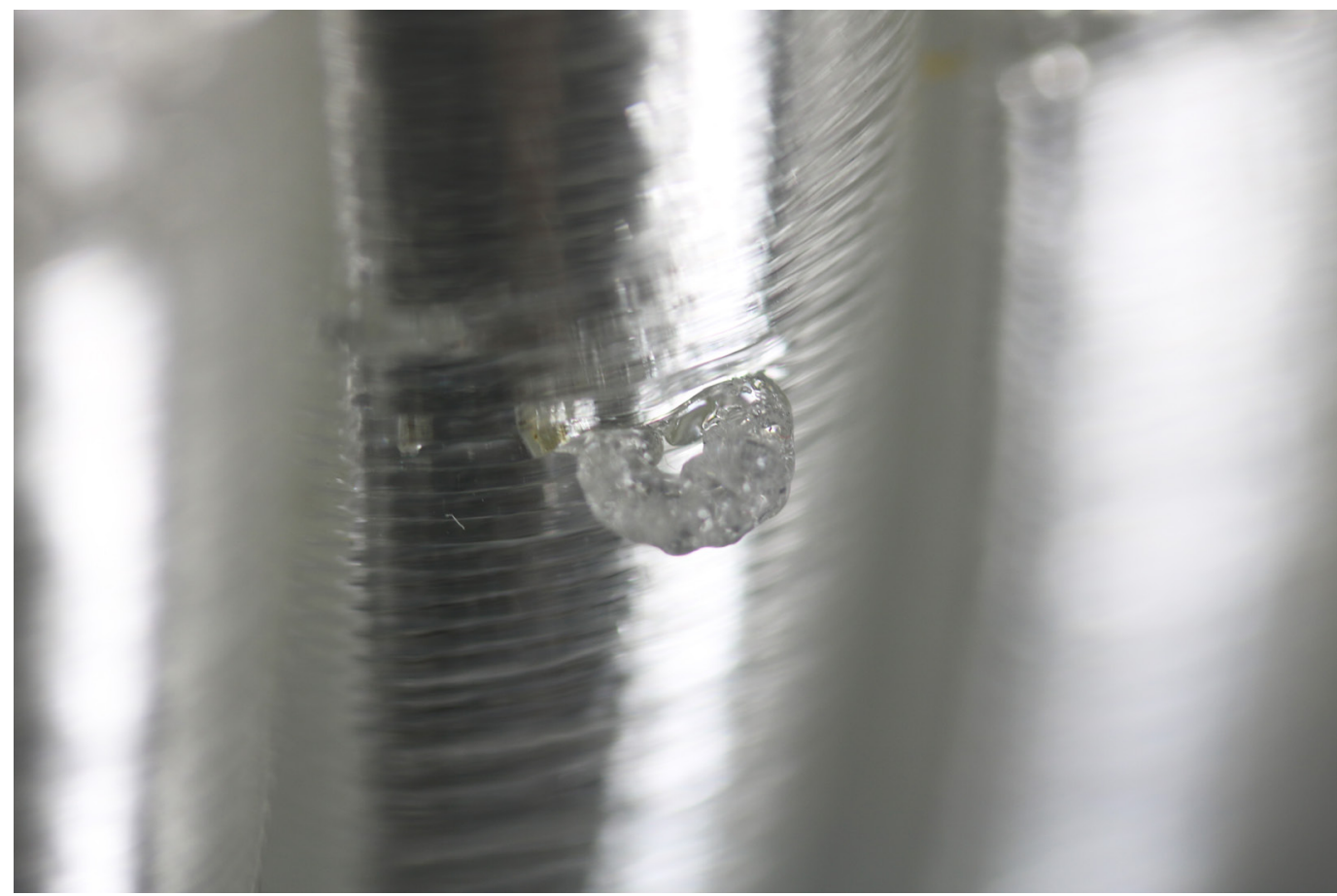

Figure 50 - Deposits of PLA within a wall of TPU. 


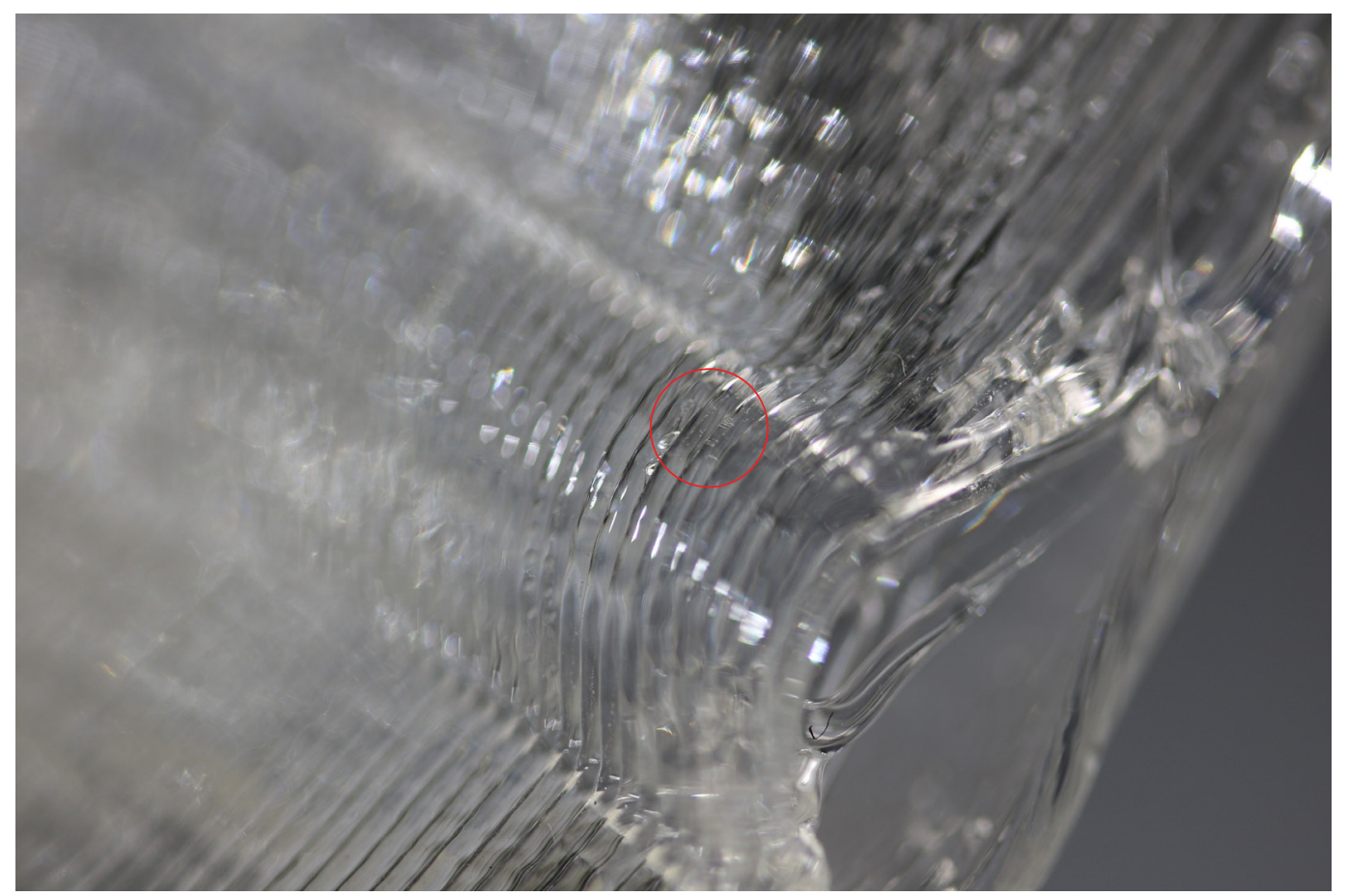

Strong and good adhesion between the layers and materials.

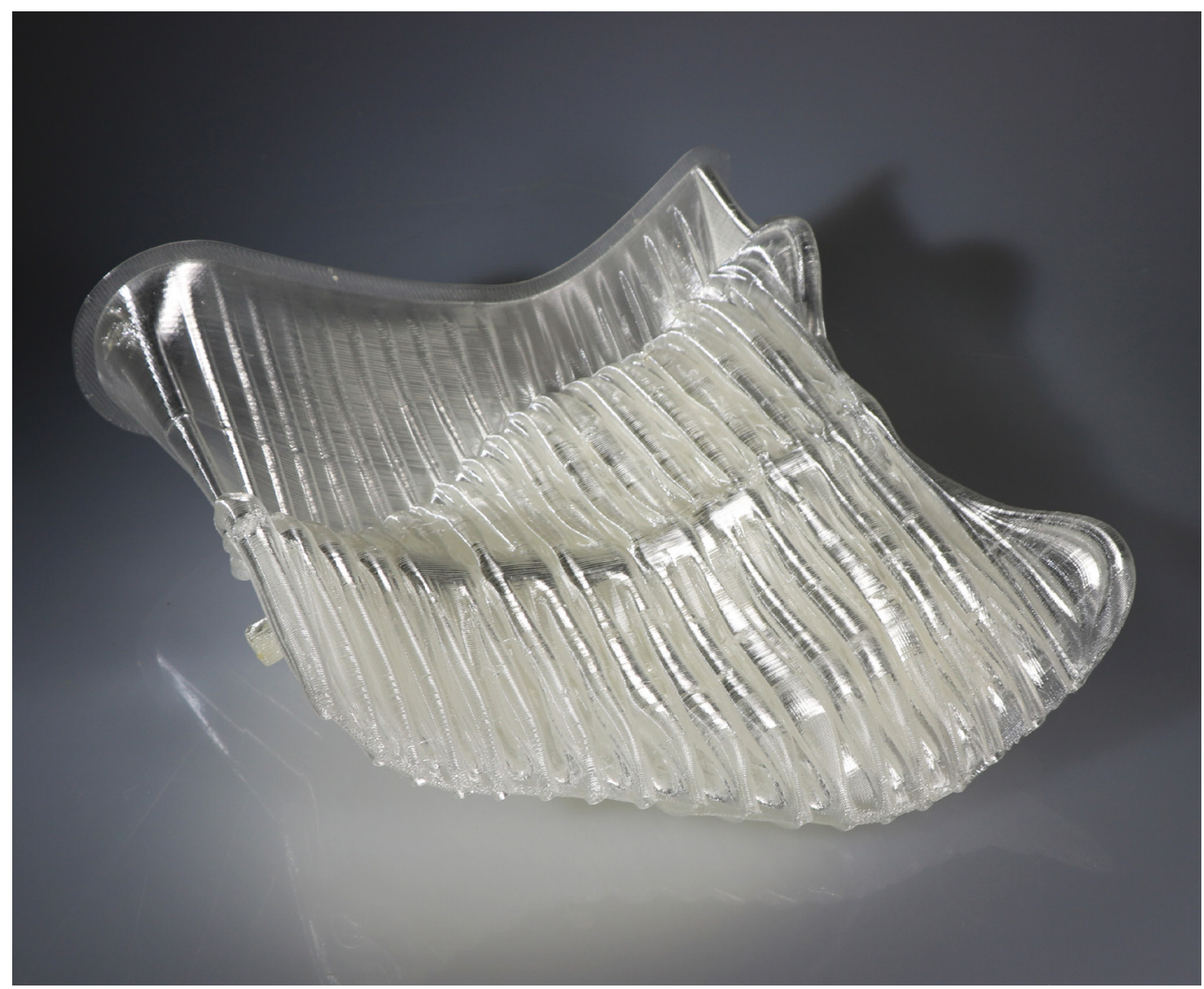

Multi-material 3D printed, Full-Scale custom motorcycle seat. 


\section{POST PROCESSING}

\section{Post Processing}

Post processing this model involved dying the plastic to a suitable colour to blend to the aesthetic of the motorcycle. Using a clear, untreated material to 3D print the seat allowed a higher quality model to be constructed. By dying the plastic afterwards allows for extensive customisation. Small scale models were used to test the dying process before dying the full scale seat.

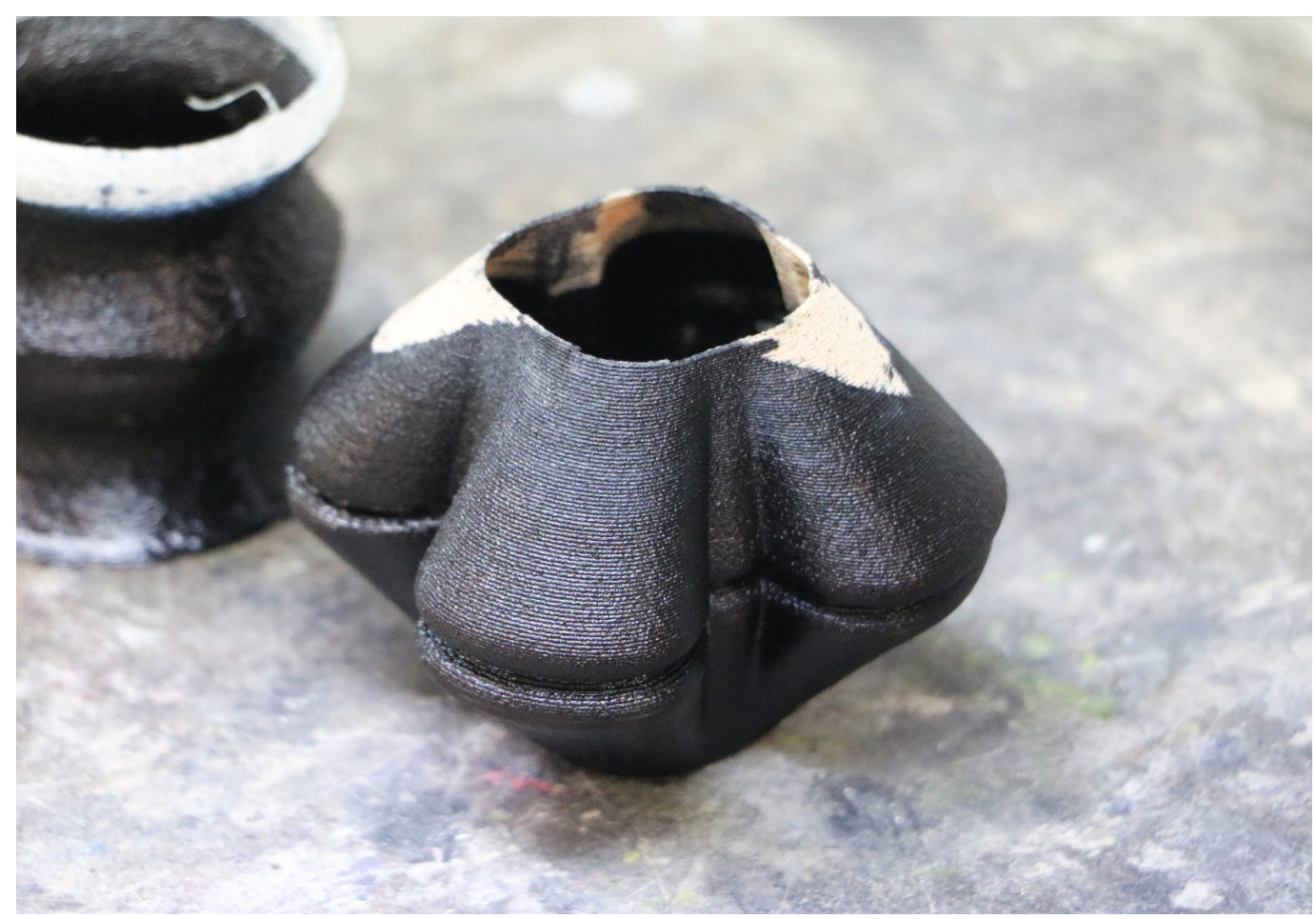

Model was partially submerged in the dye. This gave a contrast between the original colour and the dyed area. 


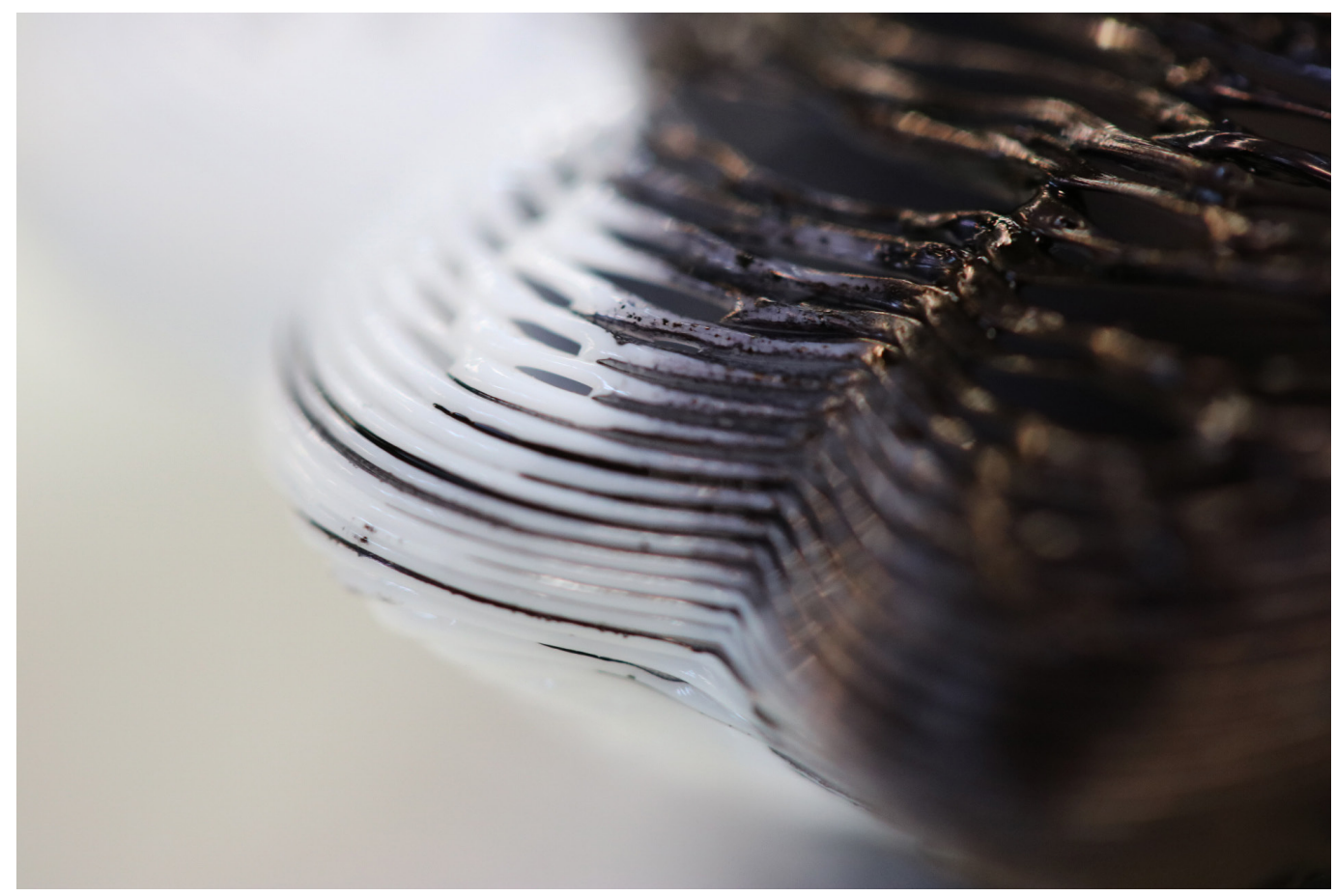

The bleed of the dye through the layer lines of the print creates an interesting effect.
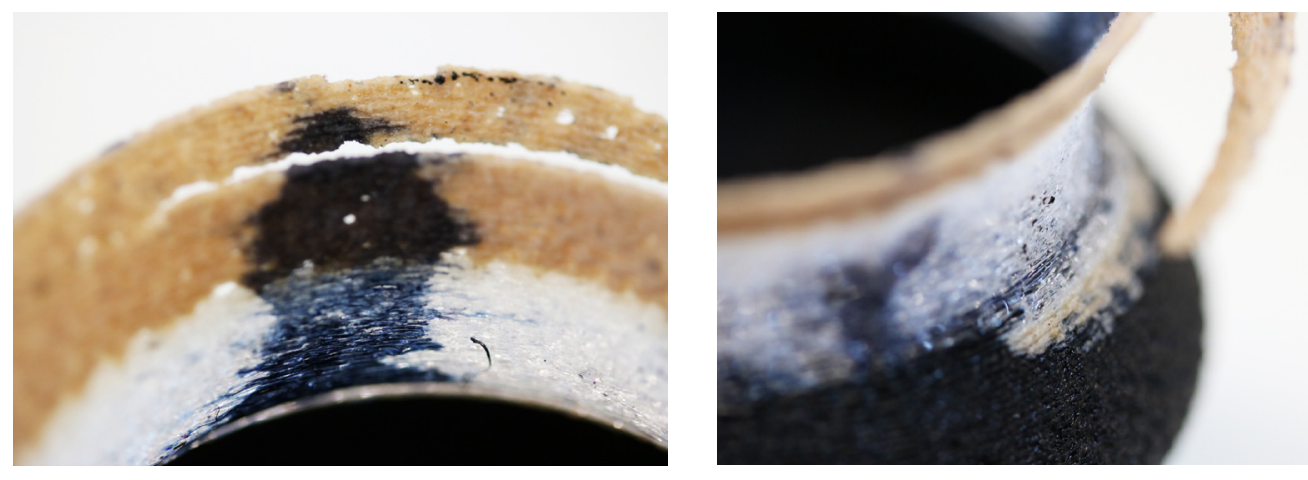

Patterns can be created using different techniques of dying the model.

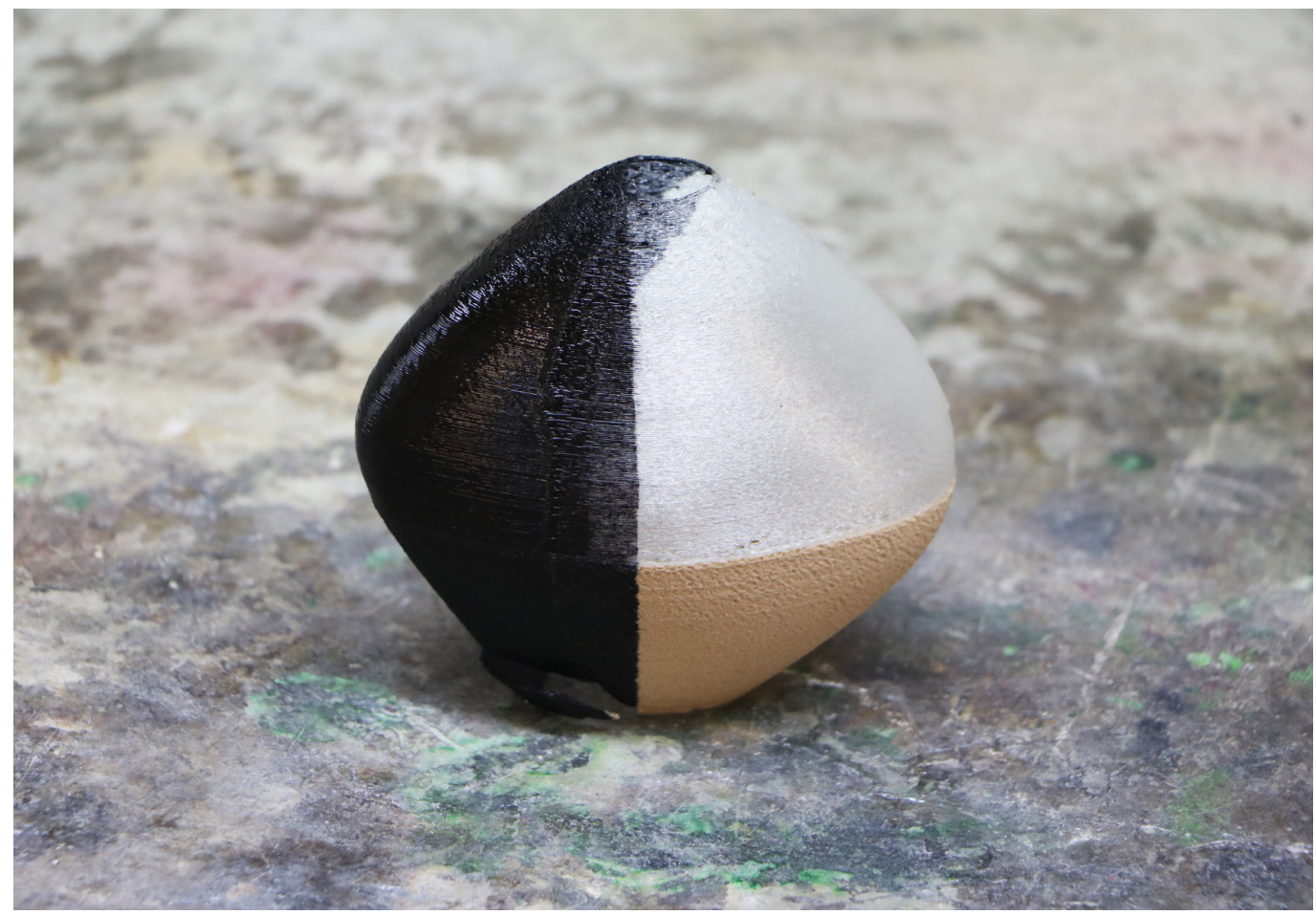




\section{Prototype 8 - Dying}

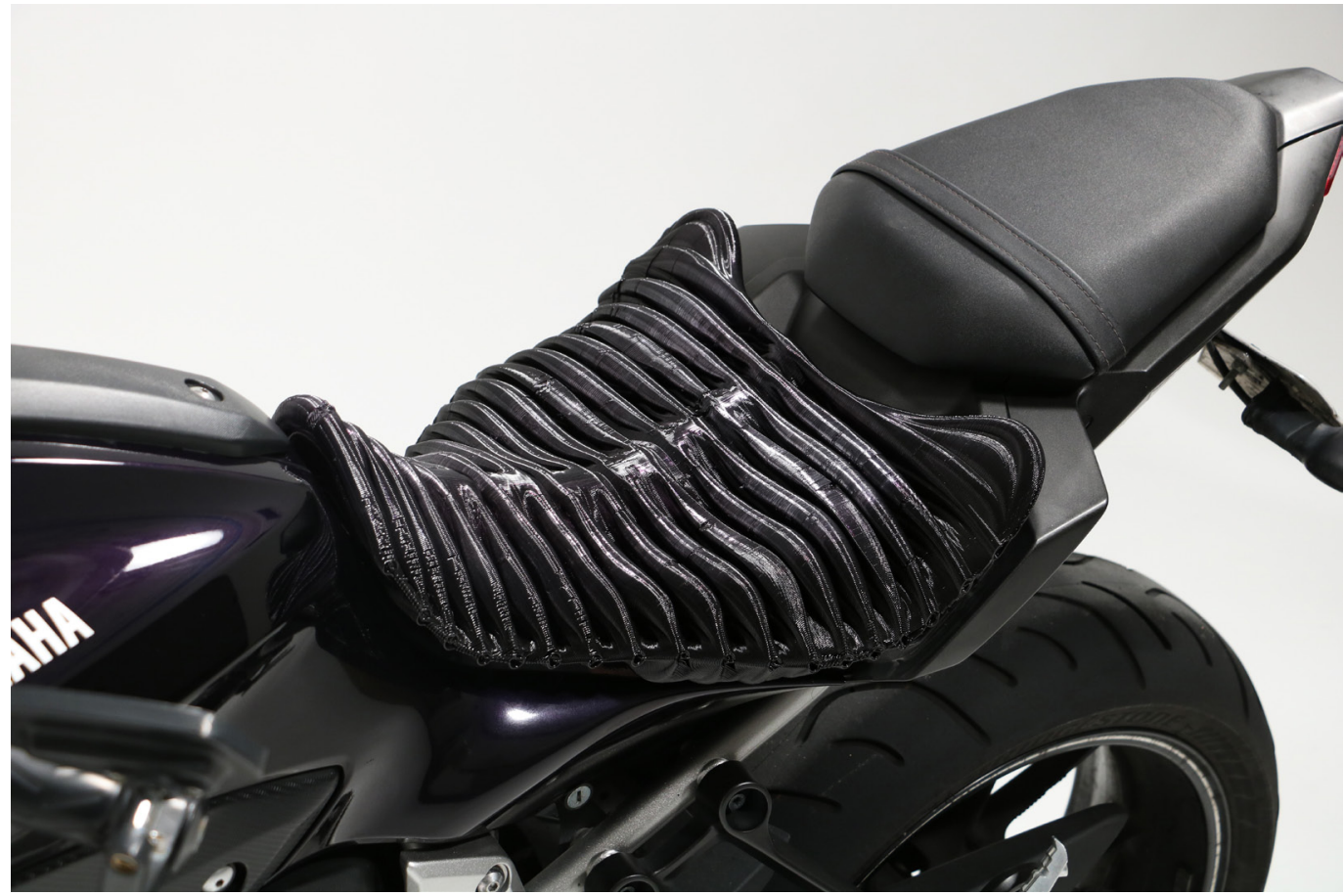

Dying Prototype 8 has revealed qualities which would not be possible by printing the seat using coloured filament. Although the model is dyed black, the transparency of the material reveals blues, purples, and magentas. The decision to dye the model rather than print using coloured filament was key in revealing these transparent qualities.

Figure 51 shows a triangular shaped piece of material extruding from the side of the model. This support is designed to help the model print. It was decided not to remove this from the final model to emphasise the 3D printing process.

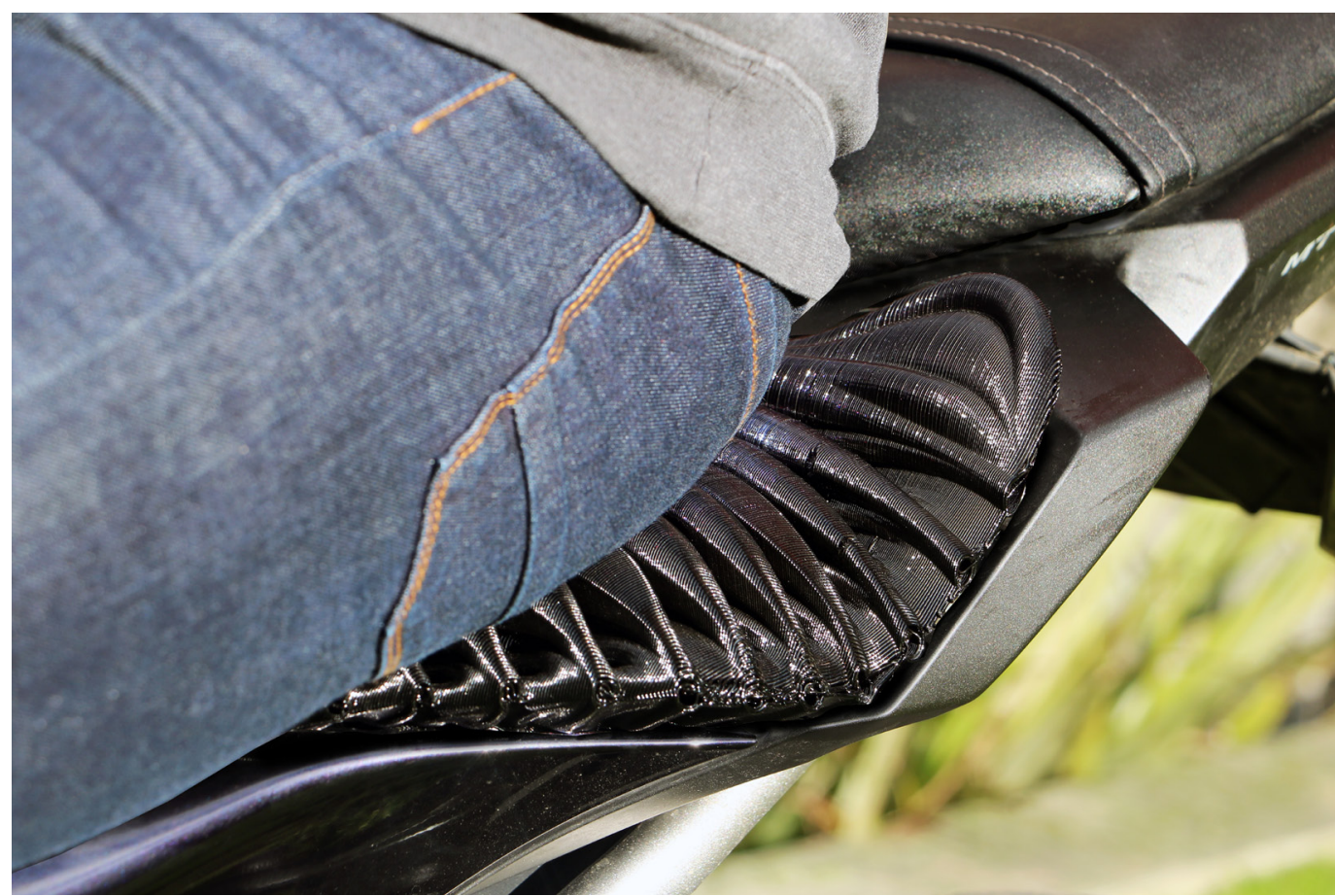




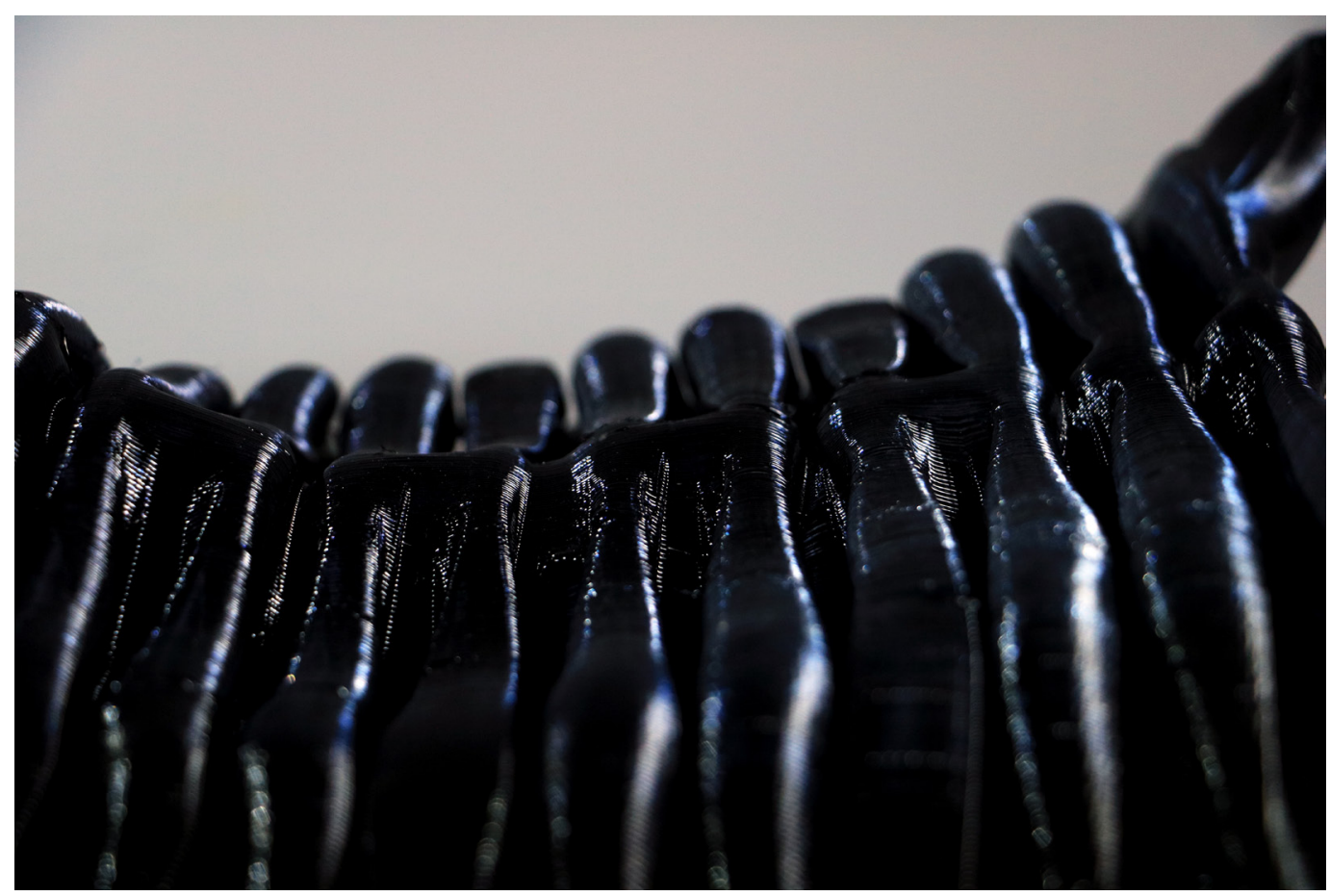

Dying the clear material black has intensified the glossiness of the surface.

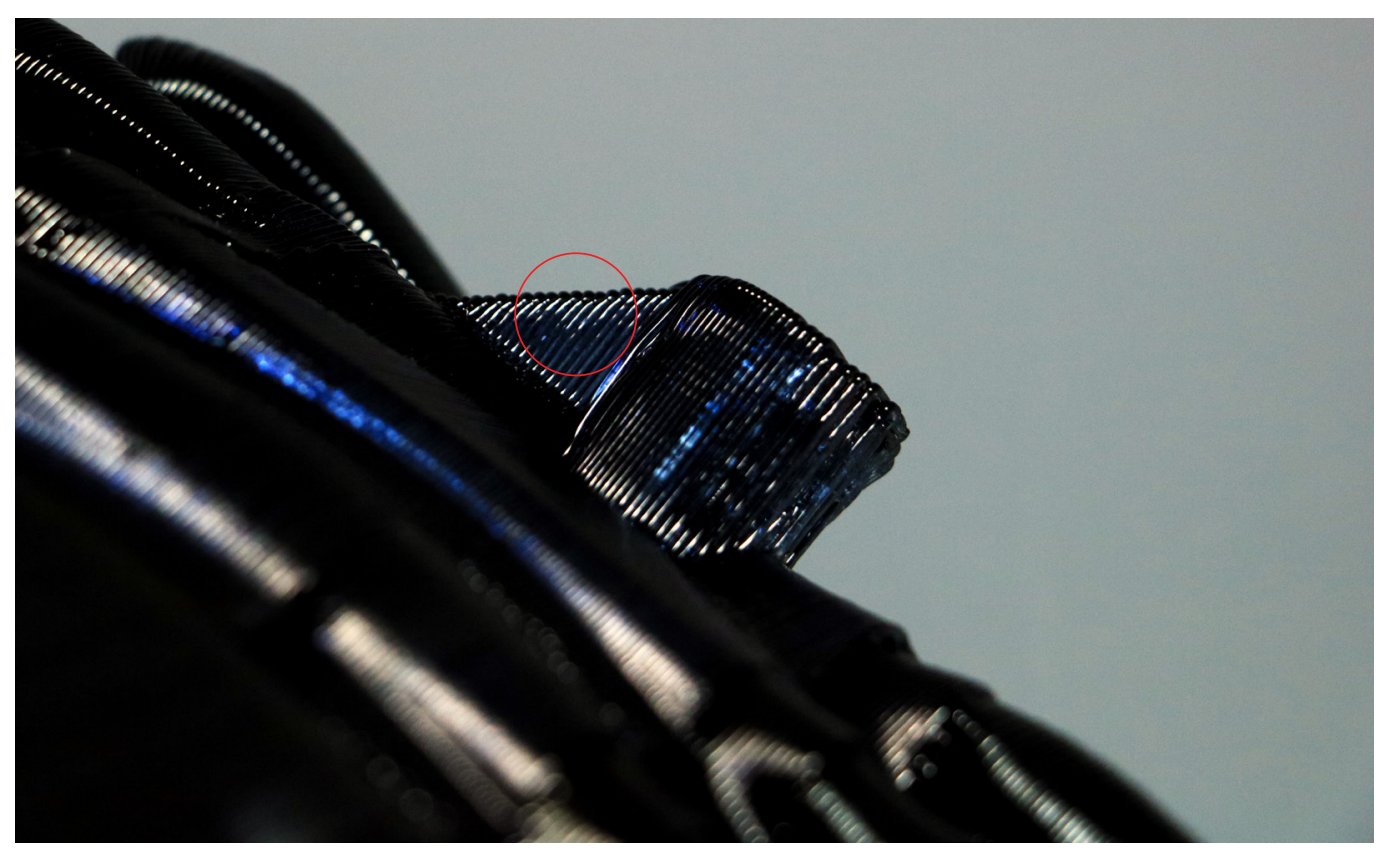

Figure 51 - Triangular support extruding from the side of the model.
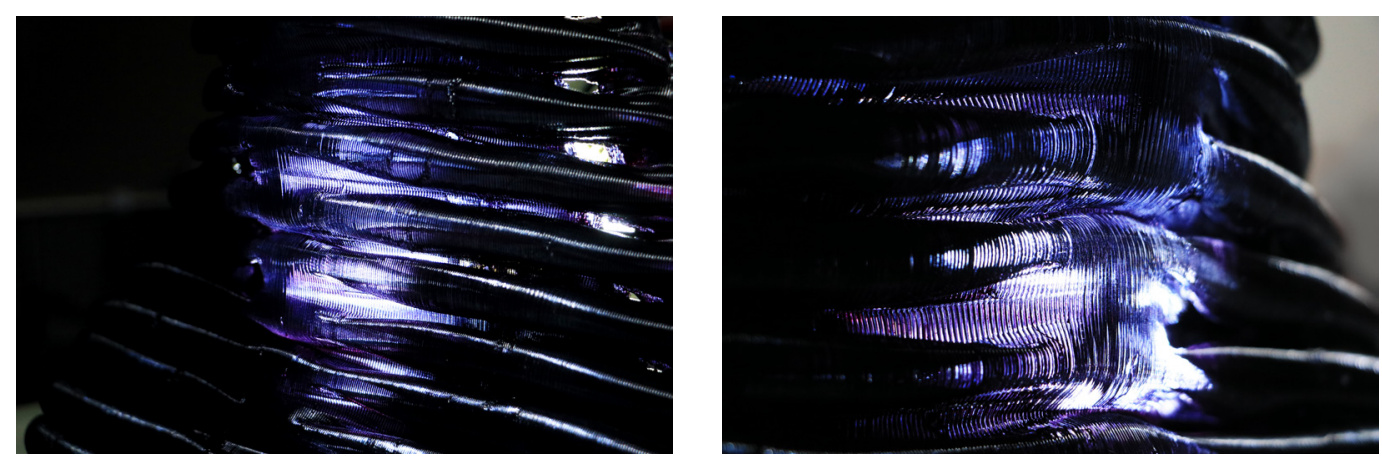

The transparency of the dyed material reveals shades of blue, purple, and magenta. 


\section{PARTICIPANT EVALUATION}

\section{Participant Evaluation}

Incorporating the body shapes of the researcher and two participants demonstrate the need for customised products. The patterns of rider's discomfort has an immediate correlation to the proportions of the riders. A taller rider suffers different patterns of discomfort to a shorter rider who tends to require a lower seat (Bust, 2009). This comparison illustrates the viability of digitally tailored seats within the motorcycle industry. The participants consist of one male and one female with a height difference of at least $50 \mathrm{~cm}$. This difference in height and gender allows for the greatest variation in comparing the effectiveness of the customization.

Participant one: Female (Blue)

Height: $155 \mathrm{~cm}$

Weight: $49 \mathrm{~kg}$

Participant two: Male (Red)

Height: $185 \mathrm{~cm}$

Weight: $105 \mathrm{~kg}$

Researcher: Male (Yellow)

Height: $181 \mathrm{~cm}$

Weight: $85 \mathrm{~kg}$

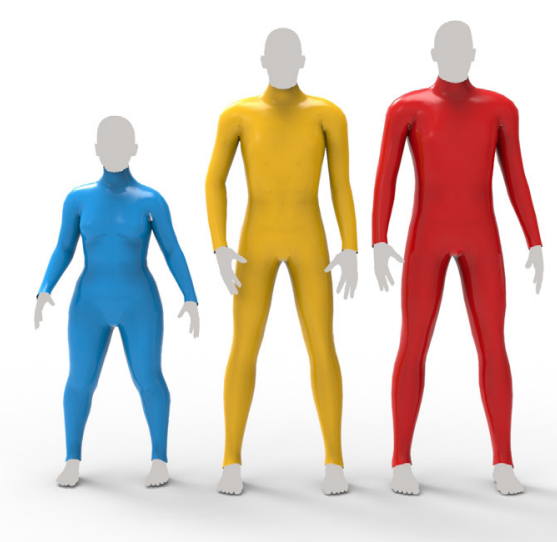

The 3D scans of the plaster castings reveal the contrast between the forms of each rider. Figure 52 \& 53 illustrates the different areas where each participant's maximum weight is distributed. The colours highlight this exertion point. It is clear that participant one's body (blue), is a lot smaller than the other two riders. Her size means that she is out of proportion to the stock motorcycle seat. It may be more comfortable having a custom fitted seat designed and shaped to her individual form and proportion.

Participant one finds it difficult to touch the ground on most motorcycles, Figure 54 shows how the structure of her seat could be two tubular structures high, lowering the seat height as much as possible. Participant one is also much lighter than the other participants so the seat does not need as much support. The structure of the other two participant's seats is thicker making it more comfortable, raising the seat height as well as giving the heavier riders more support.

It is evident that a custom motorcycle seat is an appropriate application for these technologies. Every rider has their individual body size and shape which is different to the generic seat shape that stock seats are designed around. Everyone has a unique body so the increased comfort will be provided by having a seat fitted to the individual rider's form. 


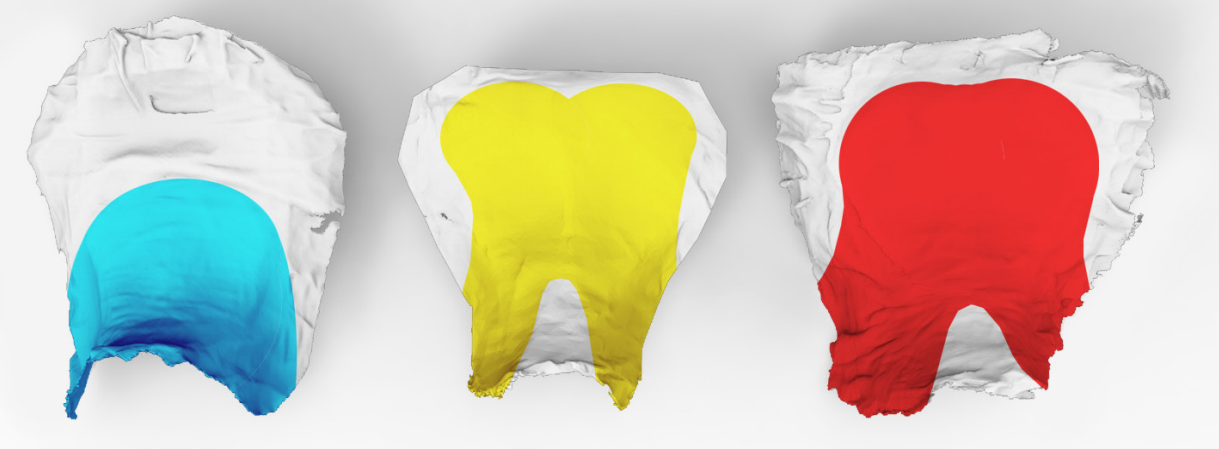

Figure 52 - Areas where each participant's maximum weight is exceeded on the plaster casting.
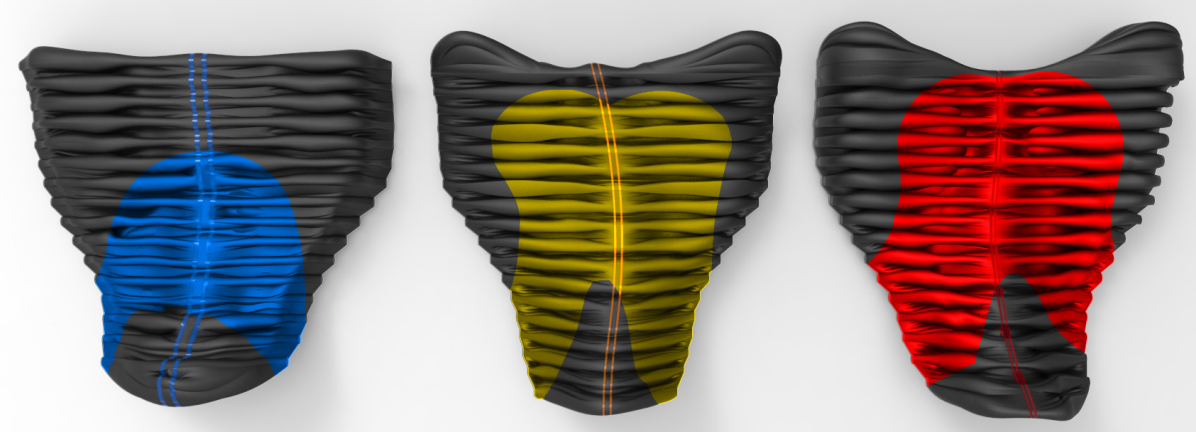

Figure 53 - Areas where each participant's maximum weight is exceeded on the seat prototypes.

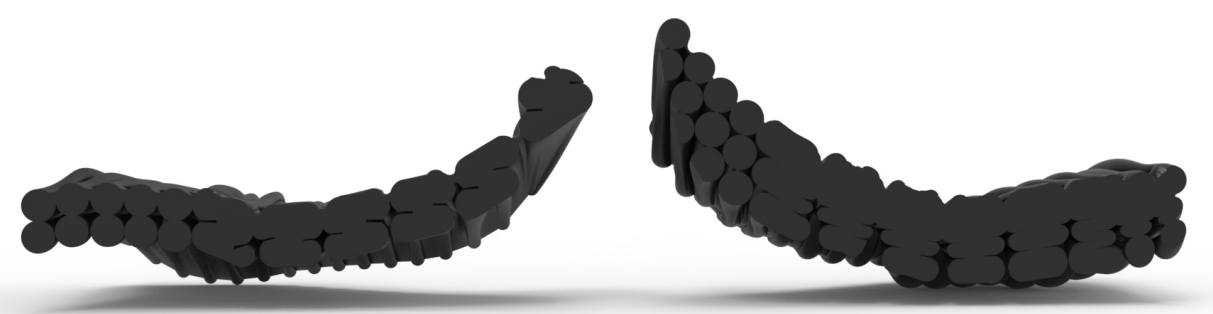

Figure 54 - The different structure heights for the different participants. 


\section{Participant Evaluation}

It is clear that the participants have different body proportions causing each individual to sit on a different part of the seat (Figure 55 \& 56). The generic, stock motorcycle seat design does not allow for this variation in body size. Having a seat that is custom designed, and fitted to the riders form, allows for an individualised level of comfort for each rider.

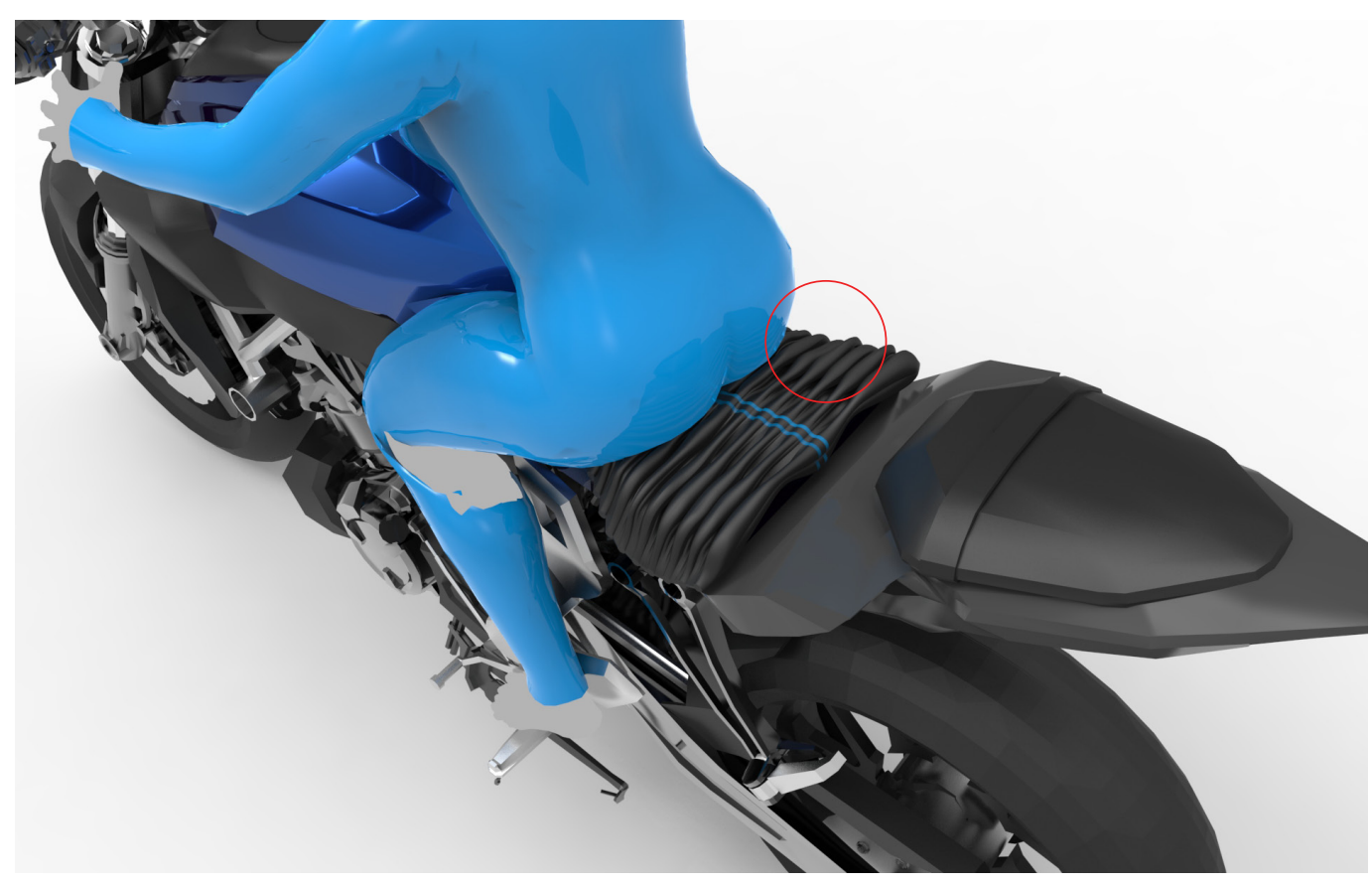

Figure 55 - Participant one's seating position.

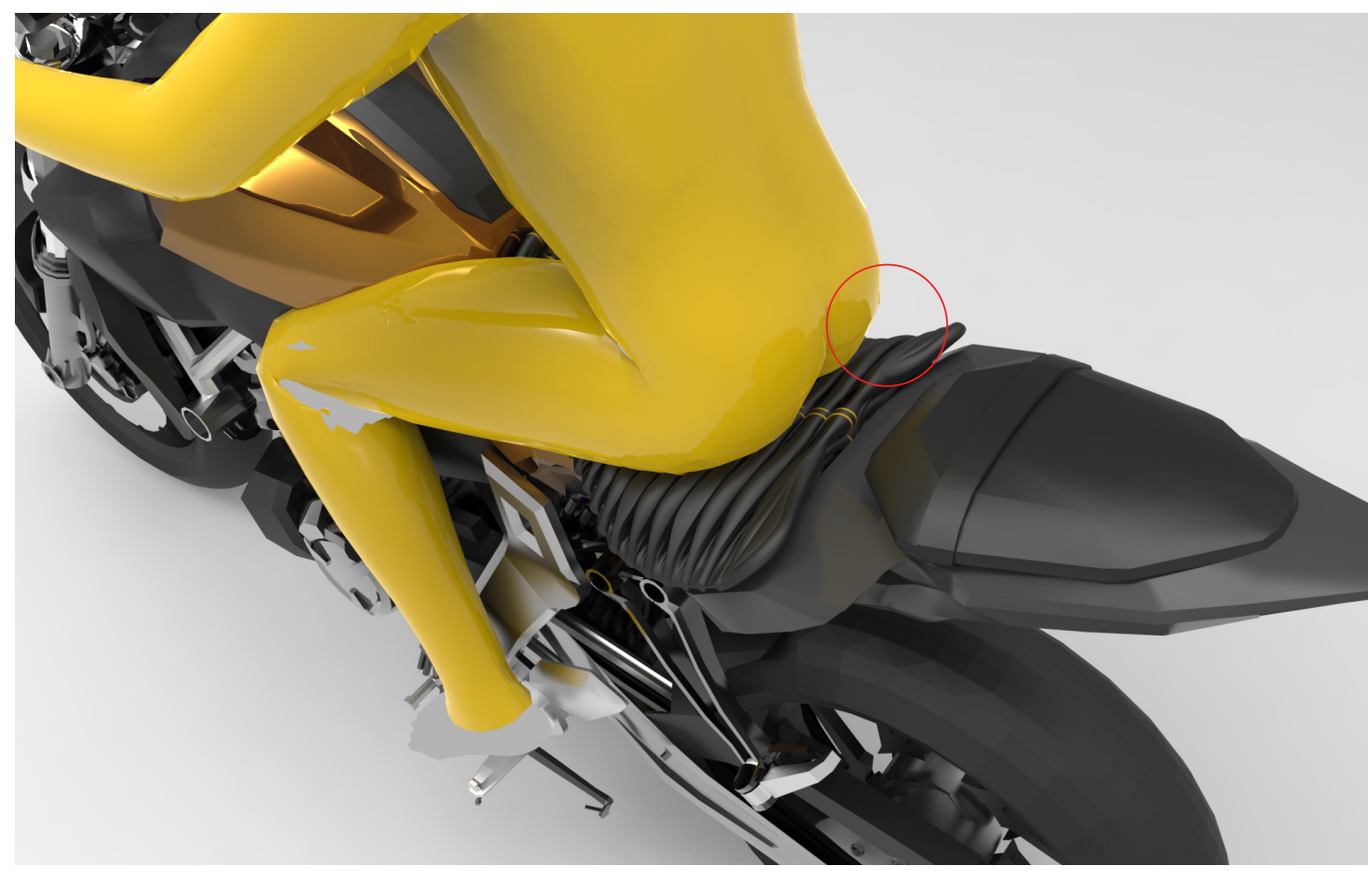

Figure 56 - Participant two's seating position 
This is a comparison emphasising not only the different areas of the seat in which each participant sits on, but also illustrating that the universal design of the motorcycle does not stop at the seat. Figure 57 shows how both riders have different body positioning whilst riding the motorcycle due to the different sizes of their body. As participant two (red) fits the size percentile the bike is designed for, he finds it comfortable to ride. Participant one (blue) is too small which leaves her uncomfortable. It is this application, where the use of 3D scanning and 3D printing technologies becomes important in the design of the motorcycle seat.
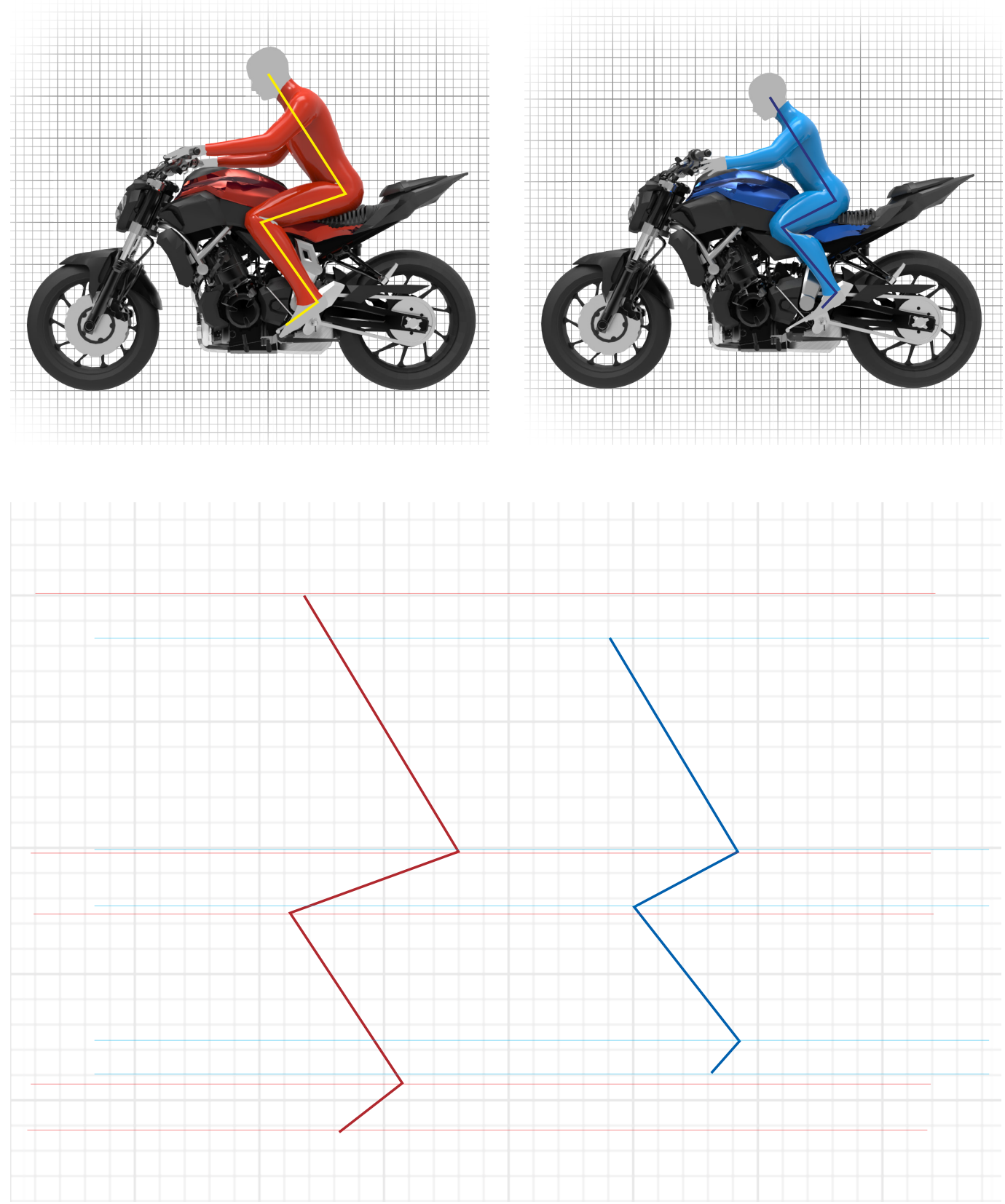

Figure 57 - Body size and positioning comparison of each rider. 


\section{Aesthetic Opportunities}

As SLA, DLP and SLS 3D printing becomes more affordable with dual-material options the opportunities to produce products with personal ergonomic values will expand. The limitation of FDM 3D printing has reduced the aesthetic possibilities within this research. The following renders are of conceptual aesthetic options where the detailed refinement of the custom seats is not limited to the capabilities of FDM 3D printing.

Figure 58 illustrates how the separate sections of these conceptual seat designs could be dualmaterial 3D printed. The hard base and supportive structure within this render are identical to prototype 8 , however, the printed skin allows for personalised variations of aesthetics to be designed. Additionally this printed skin allows for ease of cleaning and waterproofing, acting similarly to the vinyl or leather cover of traditional motorcycle seating.

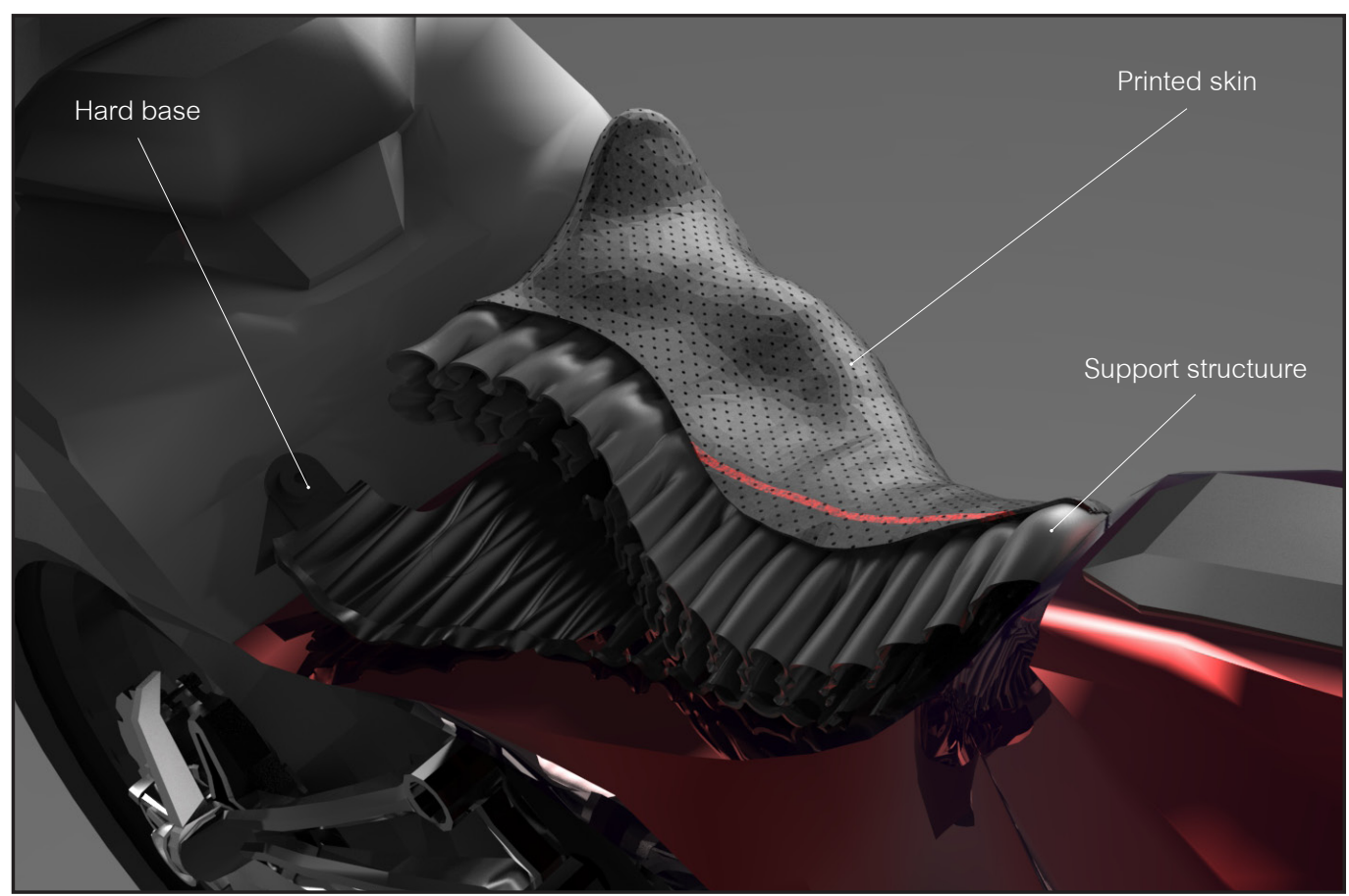

Figure 58 - Section cut of the custom seat defining the hard base, support structure, and printed skin. 


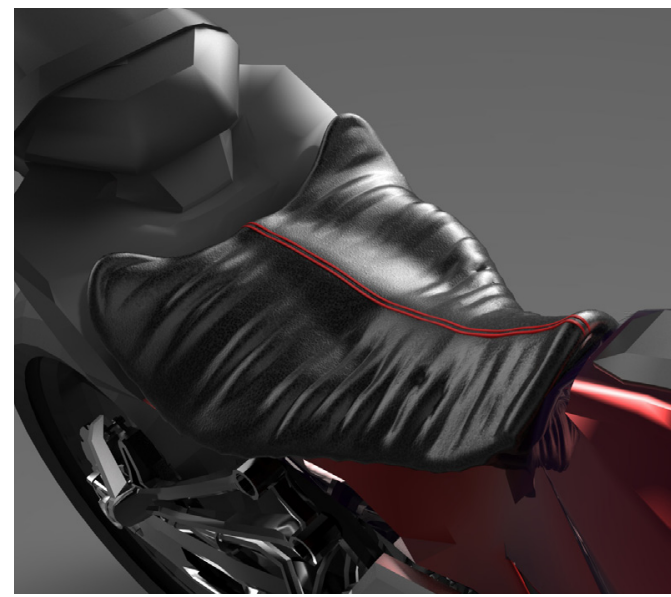

Tight skin wraps over the tubular seat. Multicoloured, striped detailing.

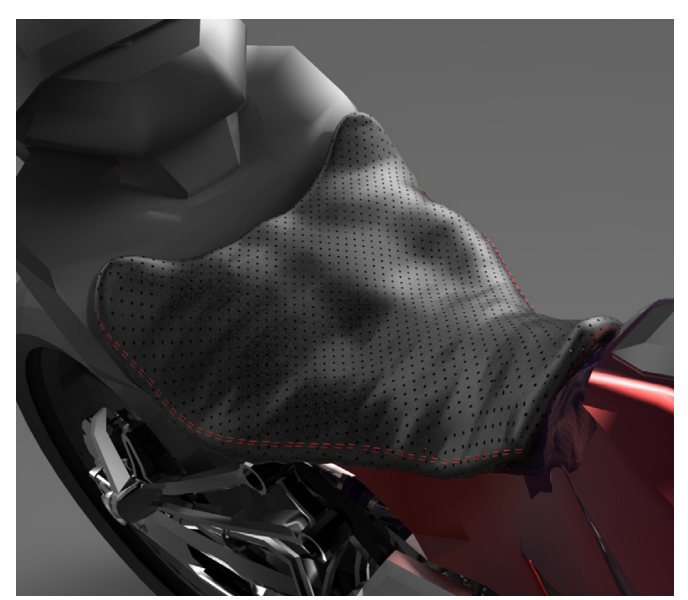

Perforated, breathable seat surface. Coloured details represent traditional upholstered seating.

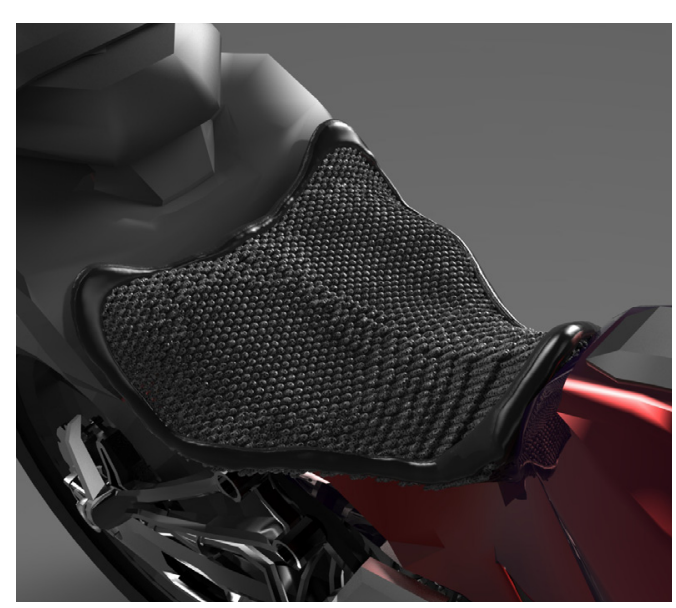

Soft tubes make up the seat form. Highly detailed dual-material.

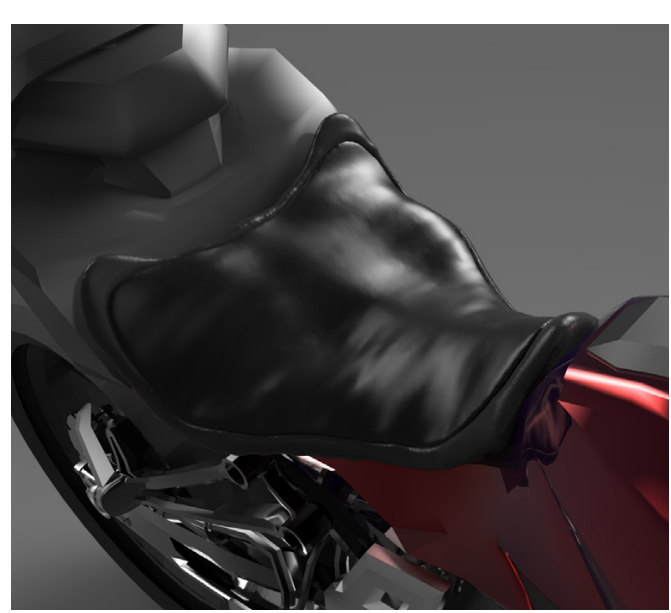

Plastic base wraps around the sides of the soft seat component.

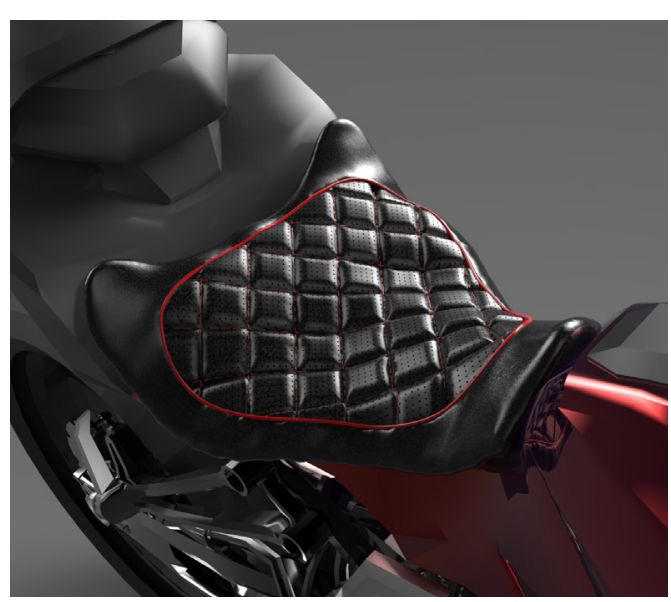

Cushioned aesthetic represents vintage motorcycle seating design.

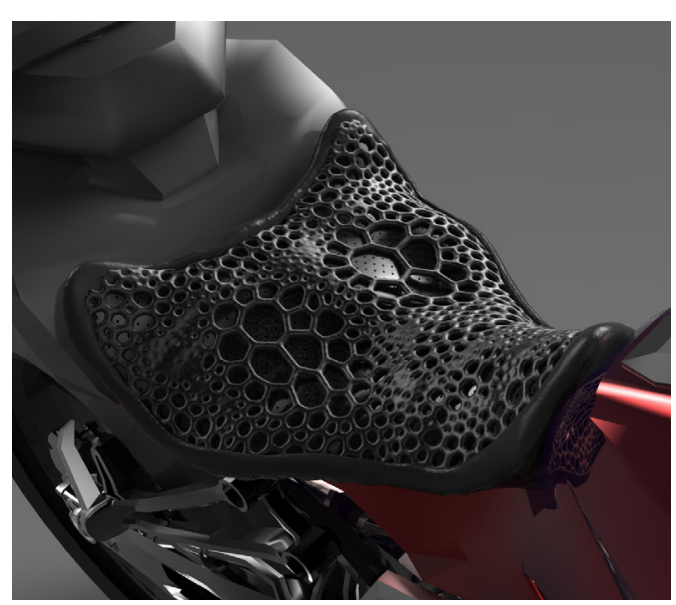

Highly detailed surface reflects the form of the rider. 


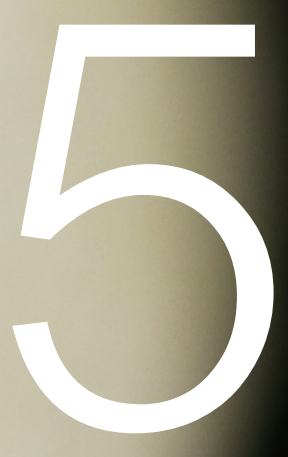

CRITICAL REFLECTION +

CONCLUSION 


\section{CRITICAL REFLECTION}

The thesis set out to apply 3D scanning and human-scale 3D printing to the design of personalised motorcycle seating. This critical reflection reviews the application, the utilisation of digital technologies, and the three-dimensional aesthetic decisions. It also reflects on how the seat functions as a real-world design prototype and considers the project's limitations.

The scope of this study is focused on creating a personal fit between an individual's body and an object. The idea of 3D printing a custom motorcycle seat which can be removed directly from the printer and mounted onto the motorcycle is an idea that connects the variability of human dimensions to the universal dimensions of a product. Motorcycle design is a domain typically serviced by making for the broadest user group, whereas the idea of a manufactured product customised to the user is unconventional and unique.

It is evident that the current way motorcycles are designed to fit the broadest percentile of human sizes is a problem. Utilising a combination of digital technologies, this research has questioned the established method of manufacture. This suggests using a design concept to develop a process which encourages customised products to satisfy the range of human scales.

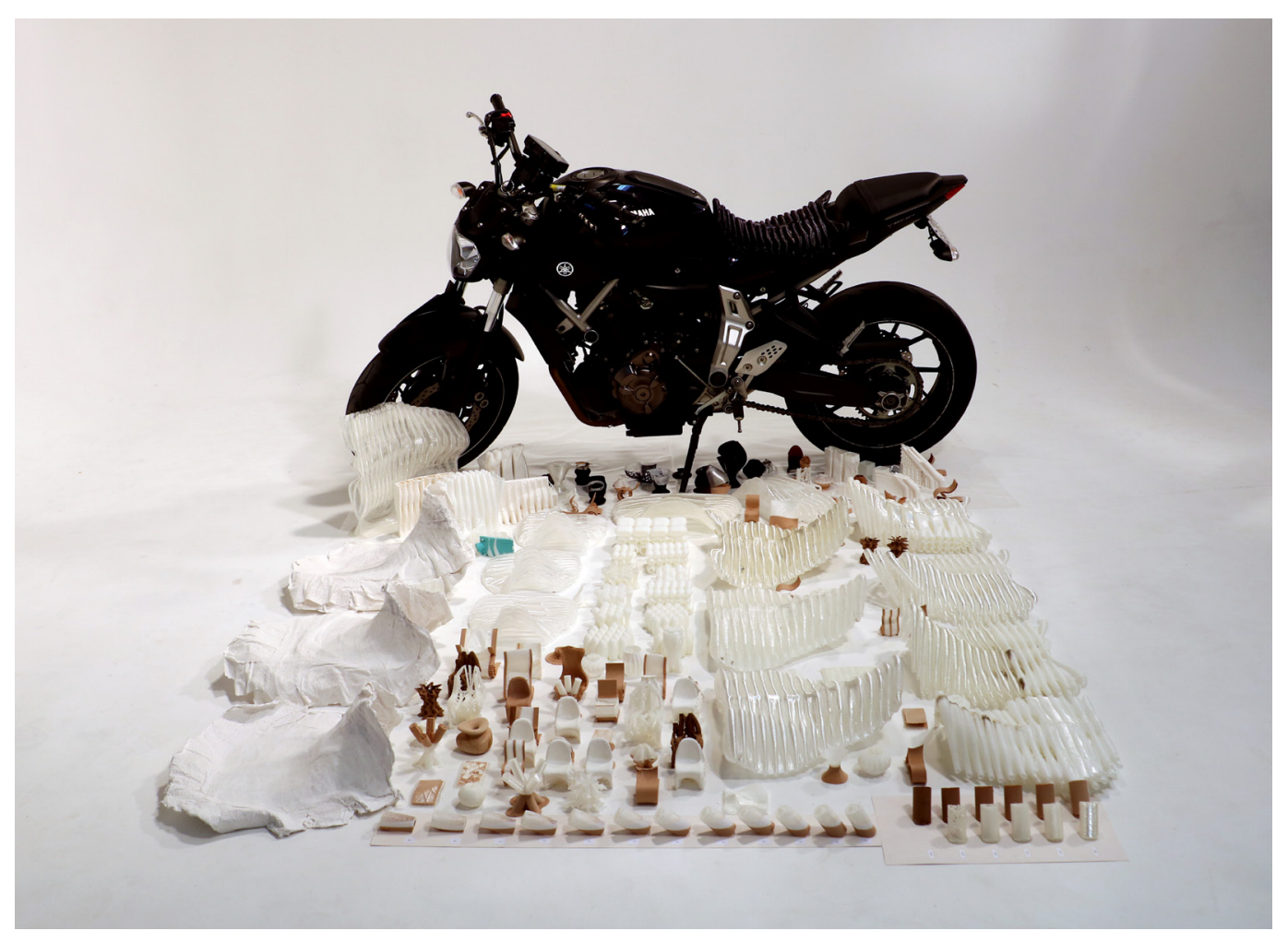

Entire series of experiments from small scale models to final prototype. 


\section{TECHNOLOGIES}

The use of 3D scanning technology has allowed for an exploration into the void between the riders form and the motorcycle. This technology has not only provided a method to capture the riders form, digitally 3D mapping it to be used in the design of the seat, but also allowed an exploration into personal ergonomics exposing the diversity of multiple rider's forms. It is this comparison which demonstrates the variation between each individual's form, ultimately conveying a need for customised products.

The benefits of customised products has always been a consumer desire, however due to traditional manufacturing methods, it remains inefficient and uneconomic. It is for this reason, large scale 3D printing technology has been utilised within this research to explore an exclusive way of manufacturing personalised goods.

From the early material explorations, to the high fidelity final prototypes, 3D printing has been employed to expand on the current technological knowledge. This experience has led to a set of 3D printing rules defined to improve, not only the final printed seat, but also the process in which the prototypes are developed. The outcome of these 3D printed prototypes is a custom motorcycle seat designed to fit an individual rider, defining a system where products are no longer bound to a generic design percentile.

\section{METHODS}

A research through design methodology has been applied throughout this study to explore and justify the use of 3D printing and 3D scanning, producing a comprehensive body of physical design experiments. Using the design methods, each prototype provides a tactile representation of digital data, rather than a hand sculpted assumption (Figure 56).

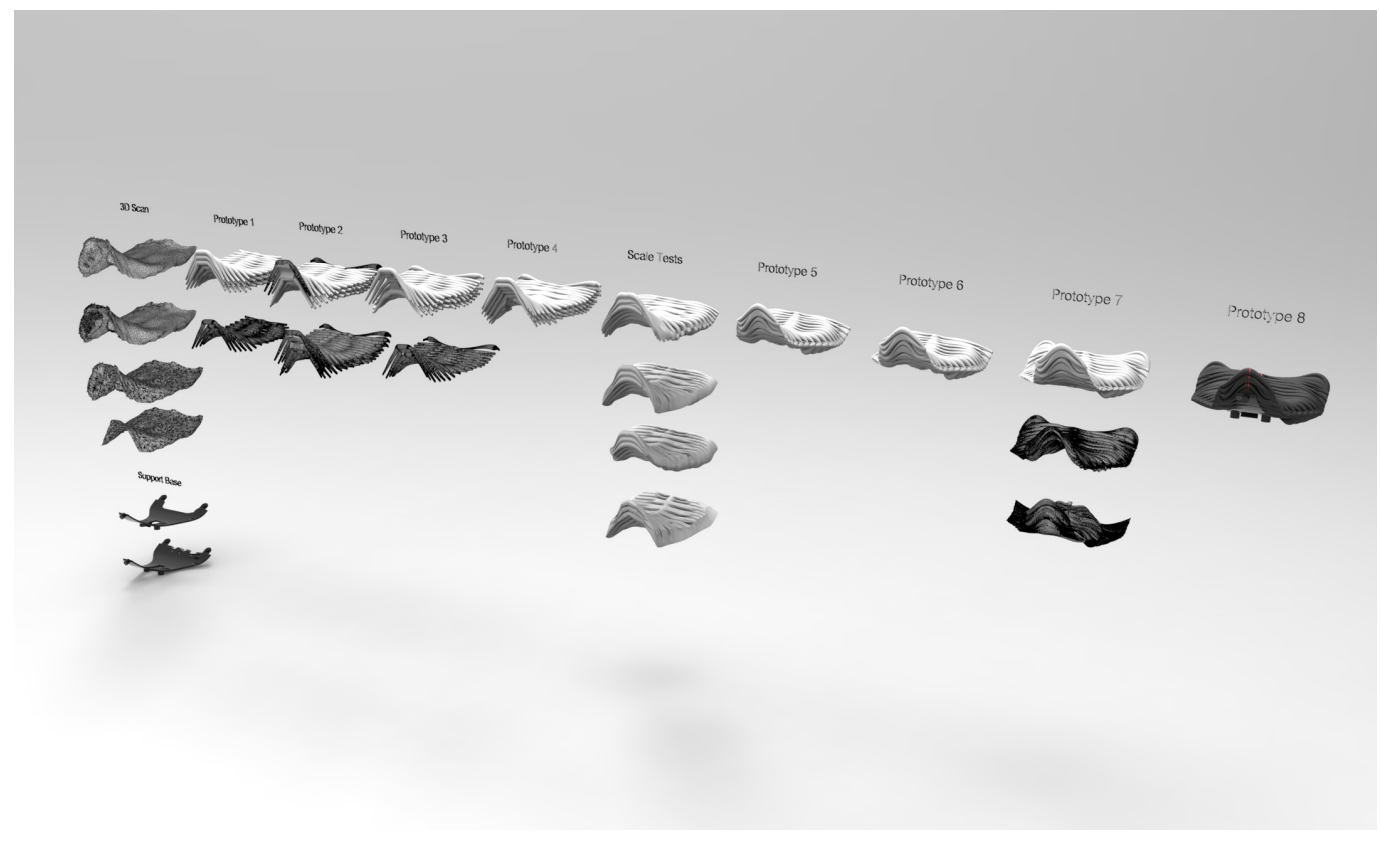

Figure 56 - The itterative design process of designing and developing the custom motorcycle seat. 
Early experiments revealed the 3D printers optimum printing parameters when producing particular three-dimensional forms. These forms, based upon successful laminations of consecutive layers, produced a set of design rules. These rules defined a set of 3D printing parameters around build angle, wall thickness, and printer speed. Alongside these, the author developed a design intuition for what would and would not work. When building the small scale cantilever seating models, an overlapping structure was developed which added support to the overhanging area (Figure 59). This support was later replicated to create support for the human body. The dynamic tests created an opportunity where the seat could be easily customised. This provided the rider with personal levels of support, in relation to their size and weight, while fitting to their individual form (Figure 60).

Prototyping seats developed a process that was used to determine an appropriate level of comfort for the rider. These prototypes reflected an aesthetic which was not influenced by precedents but derived directly from the physical qualities of the dynamic tests (Figure 61). The seat reflects the technologies used to build it. As the form of the seat is asymmetric (shaped to the body of the rider), the most efficient way of producing the seat is through the use of 3D printing technologies.

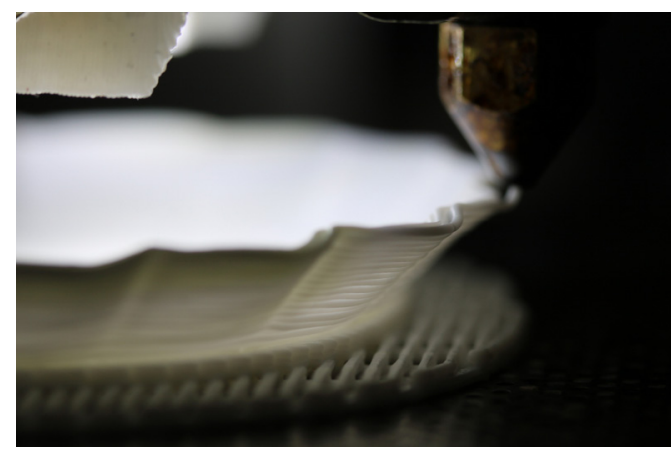

Successful laminations of consecutive layers.

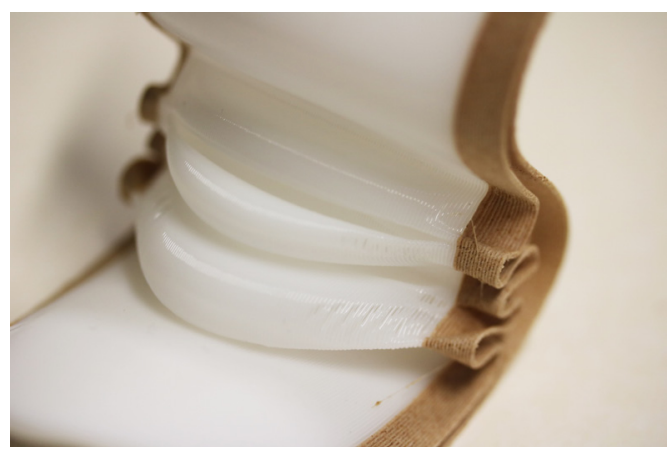

Figure 59 - Overlapping support structure.

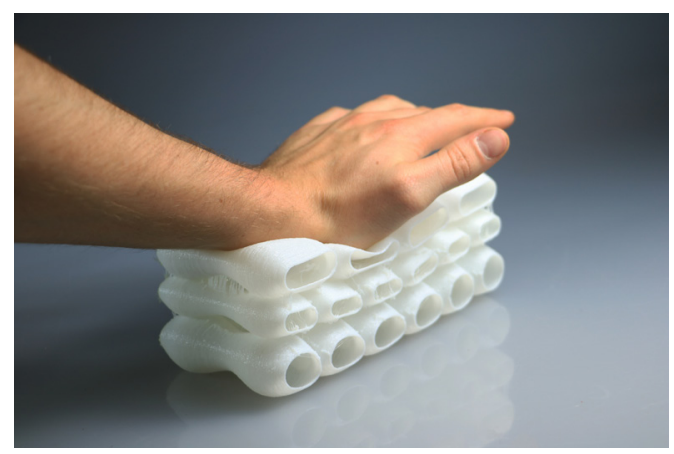

Figure 60 - Support test.

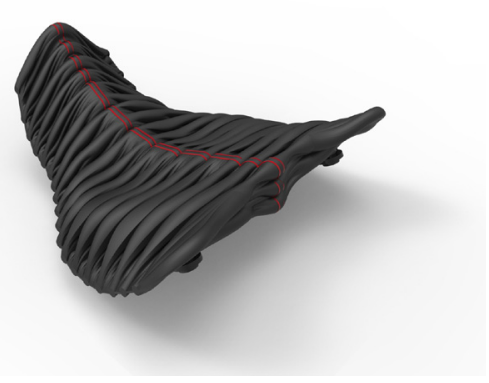

Figure 61 - Seating aesthetic derived directly from the dynamic support tests. 


\section{AESTHETIC DECISIONS}

The use of 3D printing has created an aesthetic very different to traditional upholstered seating, becoming a visual demonstration of a relatively new manufacturing technology. This aesthetic was derived from smaller support tests and the intimate knowledge of materials and printing parameters, acquired through prototyping. This strong aesthetic questions the way objects can both look, and feel, as well as how they are designed and manufactured. This suggests further opportunities where the combination of these materials and processes can be explored.

Using a sports bike as the donor motorcycle justifies the visual qualities of the seat. Figure 62 illustrates how the black panel line of the motorcycle has been used to design the seam line of the seat. There is also a visual line along the side of the seat which moves up towards the rear of the motorcycle. Incorporating both of these lines into the design accommodates the inherent form of the motorcycle. The dark colour creates harmony between seat and bike. The printed aesthetic was further explored in renders to demonstrate other visual styles.

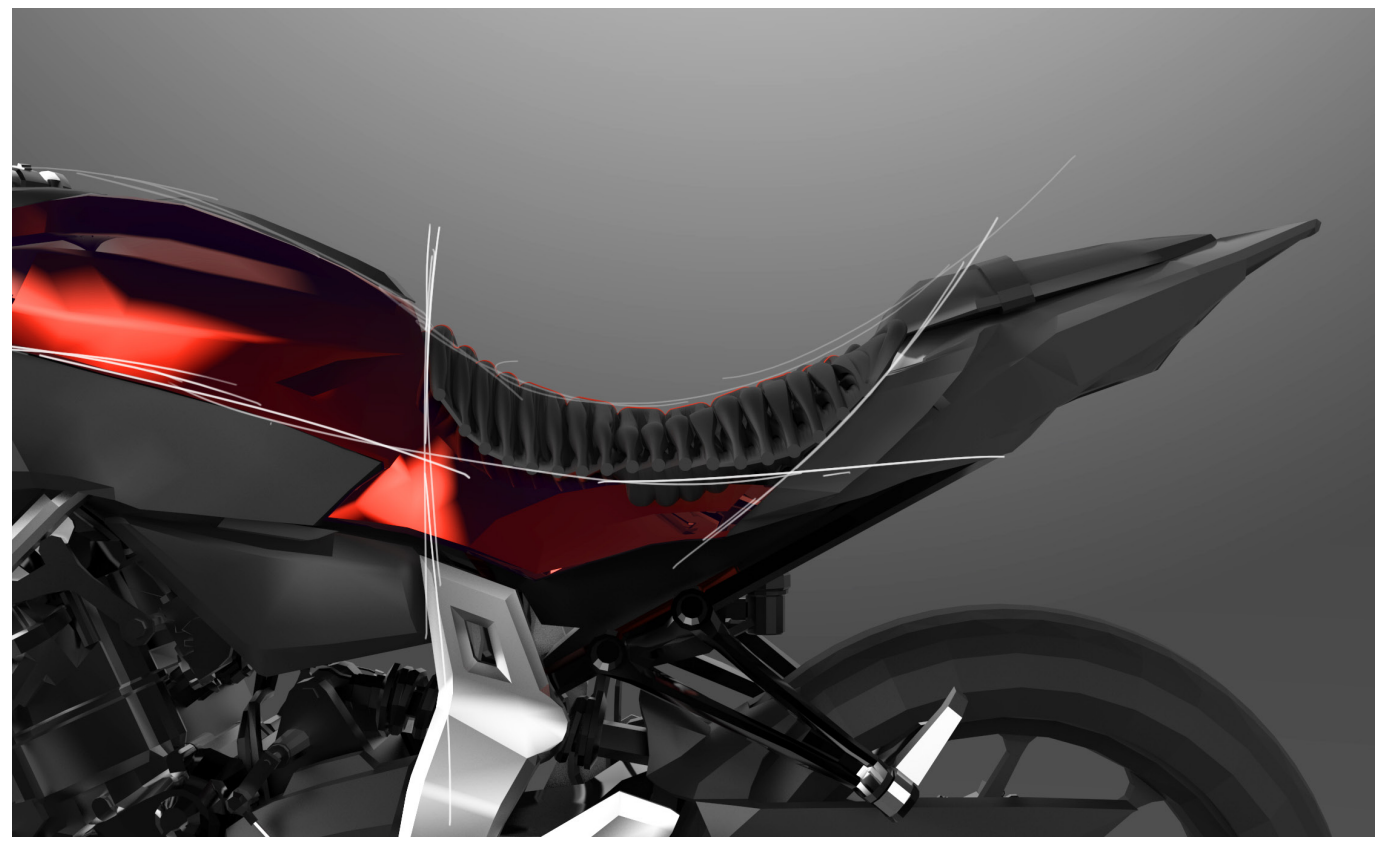

Figure 62 - The lines within the design of the motorcycle panelling are used to develop the interface between the motorcycle and the custom seat.

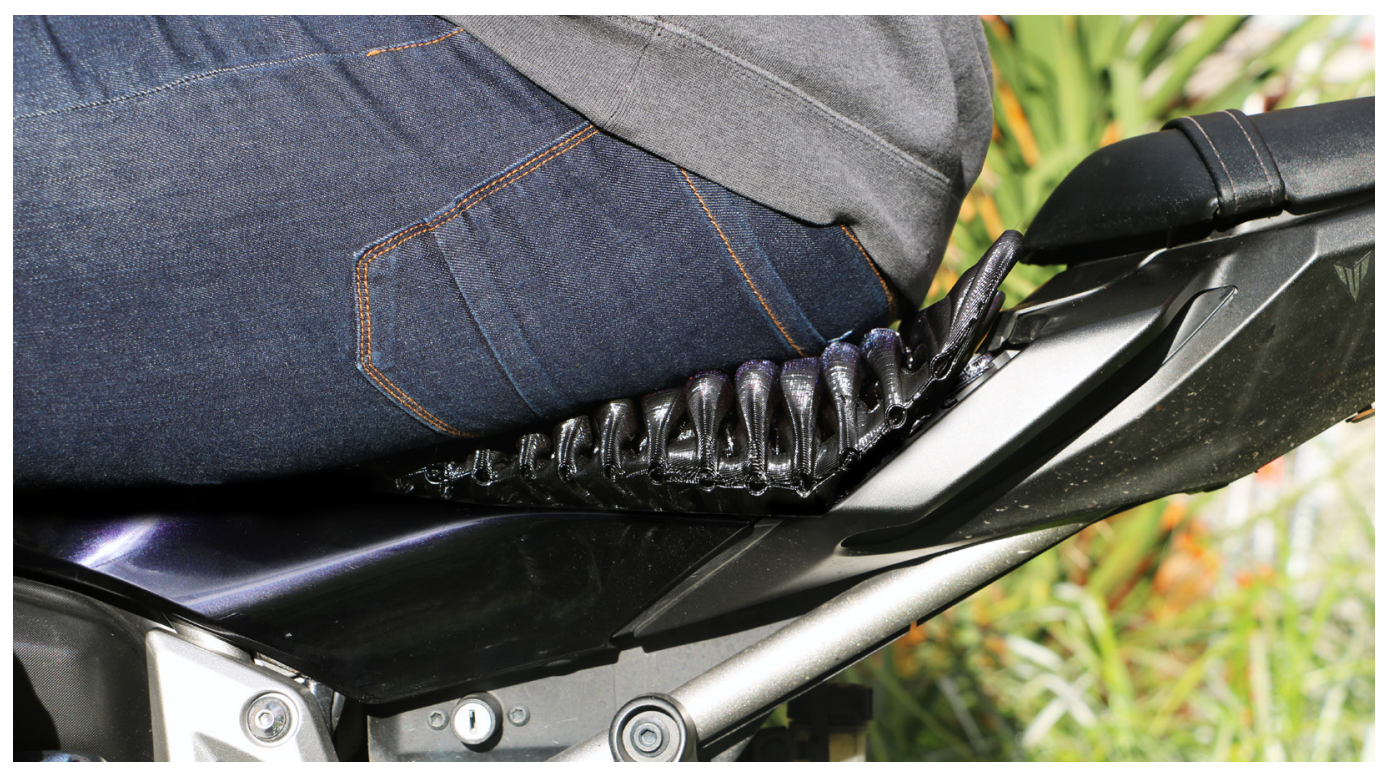

The interface between the rider, the seat, and the motorcycle. 


\section{EVALUATION}

When evaluating the full-scale prototypes, a pressure pad was used to give digital feedback emphasising how different levels of force are exerted onto areas of the seat. The pressure pad was an important tool used to develop and validate the seat prototypes, improving the fit to the rider. This equipment was provided by a furniture design studio.

It was important to keep a balance between using the pressure pad to evaluate the design of the seat and physical feedback from the rider's body. This ensured the design changes were carefully considered to reduce pressure to the rider's body.

For a short period of riding the stock seat provides enough support to make it comfortable, however over a longer period it becomes uncomfortable. When sitting on domestic furniture there is freedom to move and adjust, relieving stressed areas. When riding a motorcycle the rider is locked into a single position, unable to relieve stressed areas, causing discomfort. The custom designed seat allows the rider to spread the load of their weight over the entire surface of the seat. This distributed pressure potentially aids the rider's comfort. There is a clear contrast between the stock seat (Figure 63), and the custom 3D printed seat (Figure 64), one having extreme pressure exertion points, and the other spreading the load over the seat. This contrast emphasises the relieved stress the customised seat provides to the rider's body.

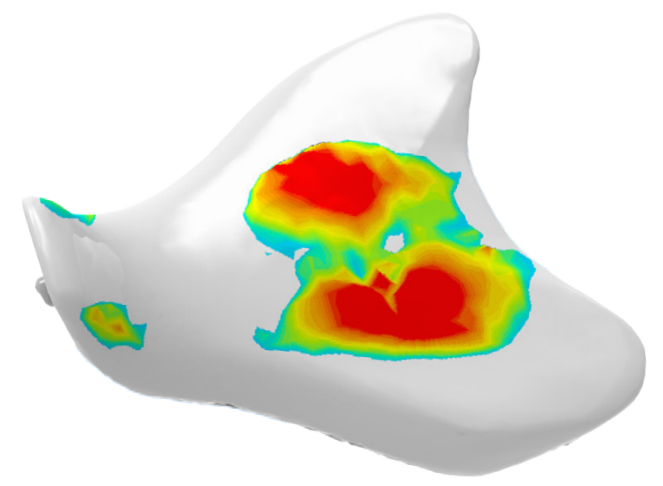

Figure 63 - The areas which receive the most pressure on the stock seat.

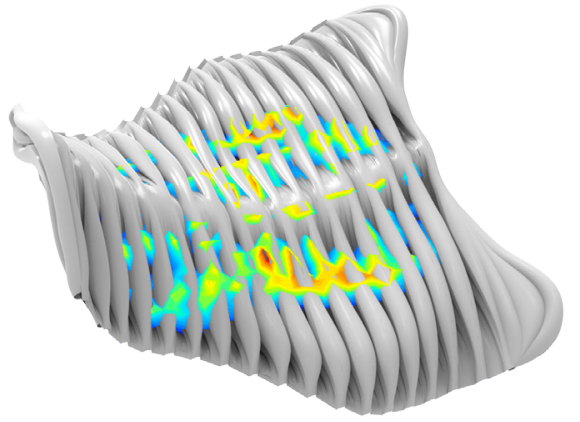

Figure 64 - The areas which receive the most pressure on the custom seat. 


\section{REAL WORLD APPLICATION}

How does the design perform as an everyday aftermarket motorcycle seat?

This seat is designed and shaped to the researcher's form so it can be road tested and developed over the course of the project. A definite difference between the custom designed seat and the stock motorcycle seat can be felt. Having a seat that was custom designed allowed its support to be tailored to the way it felt most comfortable for the researcher. When testing the final prototype, the researcher felt more comfortable on the custom 3D printed seat when compared to the stock seat. Using the stock motorcycle seat, the focus of the pain is on the rider's ischial tuberosity bones. However, with the custom seat, the discomfort is reduced due to the load being spread evenly over the surface of the rider's form.

Although the seat has reduced the discomfort, over an extended period of riding, the rider's legs, buttock, and right arm (as this is the one in control of the throttle) are locked into a position and unable to move, causing fatigue. It is this position that is at the core of the discomfort when riding a motorcycle for an extended period. The most effective way of resolving this issue would, therefore, be redesigning the entire motorcycle to fit an individual, relieving pressure in multiple areas rather than just one.

\section{LIMITATIONS AND IMPROVEMENTS}

Firstly was the transition between small and large scale 3D printing. Small 3D printers worked best for idea development rather than direct translation of technical settings.

The second limitation was testing the prototypes. As the prototype seats are shaped to the researcher, the physical testing is bias. This highlights the importance of participant testing. It would be valuable to test this seat concept getting quantitative feedback as to how the customised seat performs through the opinion of someone who is not invested in the project. This allows for further development, and refinement of the design as well as improving the process of producing customised seats for individual riders and motorcycles. The development would not only refine the method of 3D scanning the rider's form, but continue developing the 3D printing materials and technology.

It was planned to have a user group of riders evaluate the comfort levels of riding a motorcycle with a custom designed seat over an extended period. The concern with this method of testing was safety. Vehicle Testing New Zealand (VTNZ) Wellington informed the researcher that as long as the customised seat was secured correctly to the chassis of the bike using the existing bolt holes, the vehicle would be deemed safe to ride on the road. VTNZ considered the customised seat as an aftermarket motorcycle seat, therefore it was acceptable to have the rider or researcher mount the seat to the bike.

Although there are many benefits from gathering data through user testing, after considering the factors and consulting professionals, it was decided that the risks of having participants ride seats which have not been safety tested, was too high. Any incident whatsoever may bring risk to the study. 


\section{CONCLUSION}

This thesis has explored an opportunity where products can be designed and manufactured with personal aesthetic and ergonomic values. Using FDM 3D printing technology to build a customised motorcycle seat, has allowed the construction of a dynamic product concept, used to illustrate the possibilities of additive manufacturing. The design decisions made within this research, make it original, growing the body of knowledge around the capabilities of 3D printers, and the manufacturing of customised products.

This thesis set out to question mass manufactured products that selectively limit comfort. 3D printers are able to produce the one-off types of products that customer's desire but can rarely afford. The economical and time saving advantages that 3D printing offer, can create an opportunity to move the buyer away from generically designed objects, and towards personalised products that have been ergonomically fitted to their body.

This exploration has not only introduced individualised levels of comfort through the design of the motorcycle seat, but also question the traditional design process where objects are designed for the broadest user percentile. Having a custom fit motorcycle seat, designed using the rider's body, produced in an efficient and cost-effective way of increasing the rider's confidence.

Large scale multi-material 3D printing is a new method used to construct objects and products allows for 'tool less' manufacture where a product potentially can be any shape and any variation of soft and hard. The properties of flexible TPU plastic opens up many areas of design that have normally been restricted to mass-manufacturing. With progression in 3D printing technologies moving swiftly towards large scale additive manufacturing, further the opportunities for the designer, client, and customer will be made possible. 


\section{List of Figures}

Unlisted figures provided by the author of this thesis.

Figure 4. Unknown. (n.d.). The World's First 3D Printed Turbo. Retrieved from http://www. turbodynamics.co.uk/media/blog/3d-printed-turbocharger

Figure 5. Beard, L. (2017). Feetz_Lifestyle Photo. Retrieved from https://www.dropbox.com/sh/ khluxpfb7mris2I/AAAP4kCAI186H9_bA7r4QVZta?dl=0\&preview=Feetz_Lifestyle+Photo+1.png

Figure 6. Beard, L. (2017). Feetz 3D_Printing Manufacturing. Retrieved from https://www. dropbox.com/sh/khluxpfb7mris2l/AAAP4kCAl186H9_bA7r4QVZta?dl=0\&preview=Feetz_3D+Pri nting+Manufacturing.png

Figure 8. Griffiths, A. (2014). Chair by Lilian van Daal replaces upholstery with 3D-printed structure. Retrieved from https://www.dezeen.com/2014/08/05/biomimicry-3d-printed-soft-seatchair-by-lilian-van-daal/

Figure 9. Gupta, S. (n.d.). penccil : : : Flow Table. Retrieved from http://www.penccil.com/ museum.php?p=384277623695

Figure 10. Matisons, M. (2015). A Poetics of Failure: Sophie Kahn Uses 3D Printing to Challenge the Digital Era. Retrieved from https://3dprint.com/39643/sophie-kahn-deconstruction/

Figure 13. Unknown. (n.d.). Adidas Futurecraft. Retrieved from http://www.adidas.com/us/ futurecraft

Figure 17. Unknown. (n.d.). 3scn.net. Retrieved from http://3scn.net/ 
Albus, V., Bley, T. S., Borka, M., Erlhoff, M., Fogelberg, K., Gleiniger, A., ... Wijk, C. van. (2013). Modern Furniture, 150 years of design. H.f.ullmann.

Barnatt, C. (2014). 3D Printing Second Edition. Explainingthefuture.com.

Berman, B. (2012). 3-D printing: The new industrial revolution. Business Horizons, 55(2), 155-162.

Bernier, S. N., Luyt, B., \& Reinhard, T. (2014). Design for 3d printing. Maker Media.

Bogue, R. (2013). 3D printing: the dawn of a new era in manufacturing. Assembly Automation, 33(4), 307-311.

Bust, P. D. (2009). Contemporary Ergonomics 2009: Proceedings of the International Conference on Contemporary Ergonomics 2009. CRC Press.

David Bak. (2003). Rapid prototyping or rapid production? 3D printing processes move industry towards the latter. Assembly Automation, 23(4), 340-345.

Desmet, P., Overbeeke, K., \& Tax, S. (2001). Designing Products with Added Emotional Value: Development and Application of an Approach for Research Through Design. The Design Journal, 4(1), 32-47.

Unknown. (n.d.). Feetz Shoes. Retrieved from https://feetz.com/story

Gaver, W. (2012). What should we expect from research through design? In Proceedings of the SIGCHI conference on human factors in computing systems (pp. 937-946). ACM.

Unknown. (2013). Giant Bicycles begins 3D printing saddles. Retrieved from http://www.3ders. org/articles/20131118-giant-bicycles-begins-3d-printing-saddles.html

Gustke, C. (2016). Your Next Pair of Shoes Could Come From a 3-D Printer. The New York Times. Retrieved from https://www.nytimes.com/2016/09/15/business/smallbusiness/your-nextpair-of-shoes-could-come-from-a-3-d-printer.html

Gyi, D. E., \& Porter, J. M. (1999). Interface pressure and the prediction of car seat discomfort. Applied Ergonomics, 30(2), 99-107.

Unknown. (2013). Hamilton: I've fallen asleep at the wheel. Retrieved from http://www. thescottishsun.co.uk/scotsol/homepage/sport/f1/4874641/Lewis-Hamilton-news-Ive-fallenasleep-at-the-wheel-of-my-car.html

Hobson, B. (2015). Movie: Lilian van Daal's 3D-printed Biomimicry chair. Retrieved from http:// www.dezeen.com/2015/01/30/movie-lilian-van-daal-3d-printed-biomimicry-chair/ Hopkinson, N., \& Dicknes, P. (2003). Analysis of rapid manufacturing-using layer manufacturing processes for production. Proceedings of the Institution of Mechanical Engineers, Part C: Journal of Mechanical Engineering Science, 217(1), 31-39. https://doi. org/10.1243/095440603762554596

Hausfeld, H., \& Hvidberg-Hansen, P. (2013). Verner Panton, The collected works. Vitra Design Museum.

Howarth, D. (2014). Multi-material 3D-printed designs by Zaha Hadid and Francis Bitonti. 
Retrieved from http://www.dezeen.com/2014/10/28/acadia-annual-conference-3d-printeddesigns-zaha-hadid-francis-bitonti/

Unknown. (2014, April 18). Inside Koenigsegg looks at 3D printing parts for world's fastest car. Retrieved from http://www.3ders.org/articles/20140418-inside-koenigsegg-looks-at-3d-printingparts-for-world-fastest-car.html

Itoskins, S. (2014). 3D printing for artists, designers and makers. Bloomsbury Academic. Johnston, L. (2015). Digital Handmade. Thames \& Hudson.

Martin, B., \& Hanington, B. (2012). Universal Methods of Design. Rockport Publishers.

Morby, A. (2016). BMW redesigns racing wheelchair for Paralympians. Retrieved from http:// www.dezeen.com/2016/05/04/bmw-racing-wheelchair-paralympians-rio-2020/

Ochi, S. (2008). Mechanical properties of kenaf fibers and kenaf/PLA composites. Mechanics of Materials, 40(4-5), 446-452.

Ong, M. H., Wagner, H., Tuck, C., \& Hague, R. (2008). Body-fitting customisation of motorcycle seats: an investigation of consumer requirements. International Journal of Mass Customisation, 2(3/4), 375.

Pina, L. (2000). Fifties Furniture (2nd ed.). Schiffer.

Post, E. (2015). Cost Effective 3D Printing, Thanks to Mass Customization. Retrieved from https://www.typeamachines.com/blog/cost-effective-3d-printing-thanks-to-mass-customization Robertson, S. A., \& Minter, A. (1996). A study of some anthropometric characteristics of motorcycle riders. Applied Ergonomics, 27(4), 223-229.

Schubert, C., Langeveld, M. C. van, \& Donoso, L. A. (2013). Innovations in 3D printing: a 3D overview from optics to organs. British Journal of Ophthalmology.

Seidenstücker, T., \& Fritz, H.-G. (1998). Innovative biodegradable materials based upon starch and thermoplastic poly(ester-urethane) (TPU). Polymer Degradation and Stability, 59(1), 279-285.

Stimpson, M. (1987). Modern Furniture Classics. The architectural press: Iondon.

Unknown. (n.d.). The World's First 3D Printed Turbo. Retrieved from http://www.turbodynamics. co.uk/media/blog/3d-printed-turbocharger

Thompson, R. (2007). Manfacturing processes for design professionals. Thames \& Hudson. Unknown. (2017). Vitra | Panton Chair. Retrieved from https://www.vitra.com/en-us/product/ panton-chair

Wohlers, T. (2014). 3D printing and additive manufacturing state of the industry and world wide progress report. Wohlers Report 2014.

Watson, A. (2002). Mod to Memphis, design in colour 1960s-80s. Powerhouse publishing. Zimmerman, J., Forlizzi, J., \& Evenson, S. (2007). Research through design as a method for interaction design research in $\mathrm{HCl}$. In Proceedings of the $\mathrm{SIGCHI}$ conference on Human factors in computing systems (pp. 493-502). ACM. 
MEMORANDUM

Phone $\quad 0-4-4635480$

Email suran.corbettivuwacma

\begin{tabular}{|c|c|}
\hline TO & Alex Christensen \\
\hline COPY TO & $\begin{array}{l}\text { Bernard Guy } \\
\text { Ross Stevens }\end{array}$ \\
\hline FROM & AProf Susan Corbett, Convener, Human Ethics Committee \\
\hline DATE & 16 March 2017 \\
\hline PAGES & 1 \\
\hline SUBJECT & $\begin{array}{l}\text { Ethics Approval: } 24126 \\
\text { Portraits through products. } \\
\text { An exploration into Personalised motorcycle seating designed to } \\
\text { improve comfort and desirability. }\end{array}$ \\
\hline
\end{tabular}

Thank you for your application for ethical approval, which has now been considered by the Standing Committee of the Human Ethics Committee.

Your application has been approved from the above date and this approval continues until 10 July 2017 . If your data collection is not completed by this date you should apply to the Human Ethics Committee for an extension to this approval.

Best wishes with the research.

Kind regards

Susan Corbett

Convener, Victoria University Human Ethics Committee 


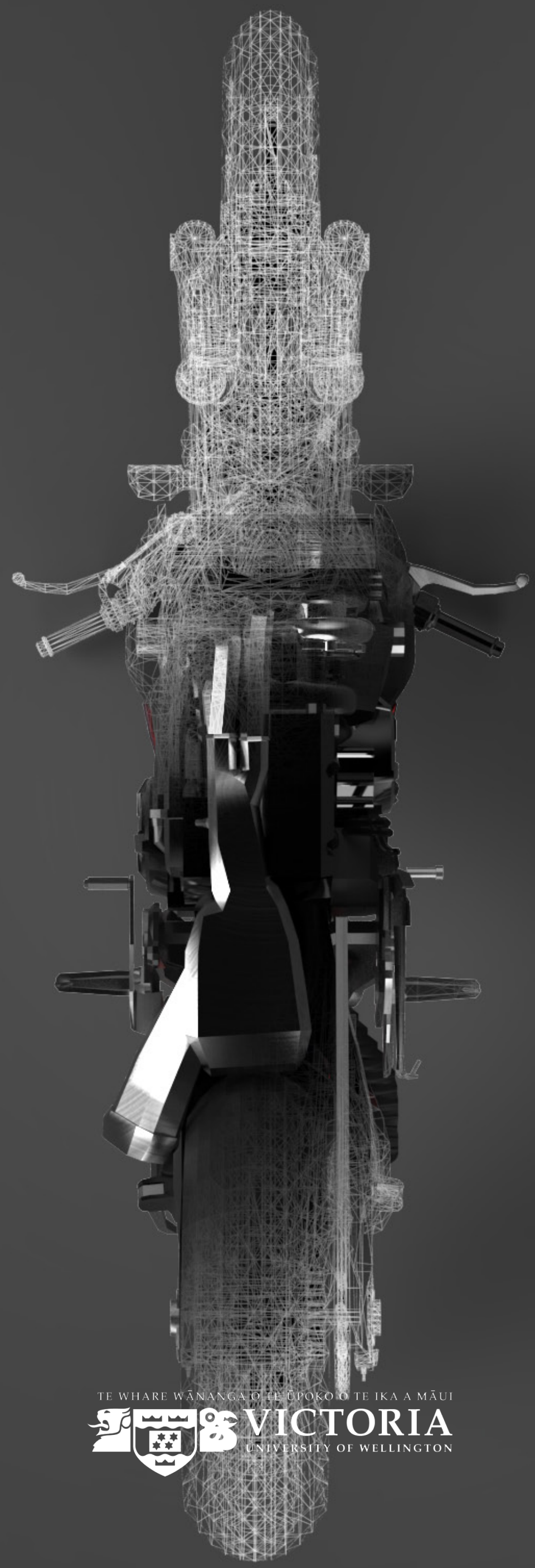

